Prepared in cooperation with the

Northern Tier Regional Planning and Development Commission

\title{
Drinking Water Health Standards Comparison and Chemical Analysis of Groundwater for 72 Domestic Wells in Bradford County, Pennsylvania, 2016
}

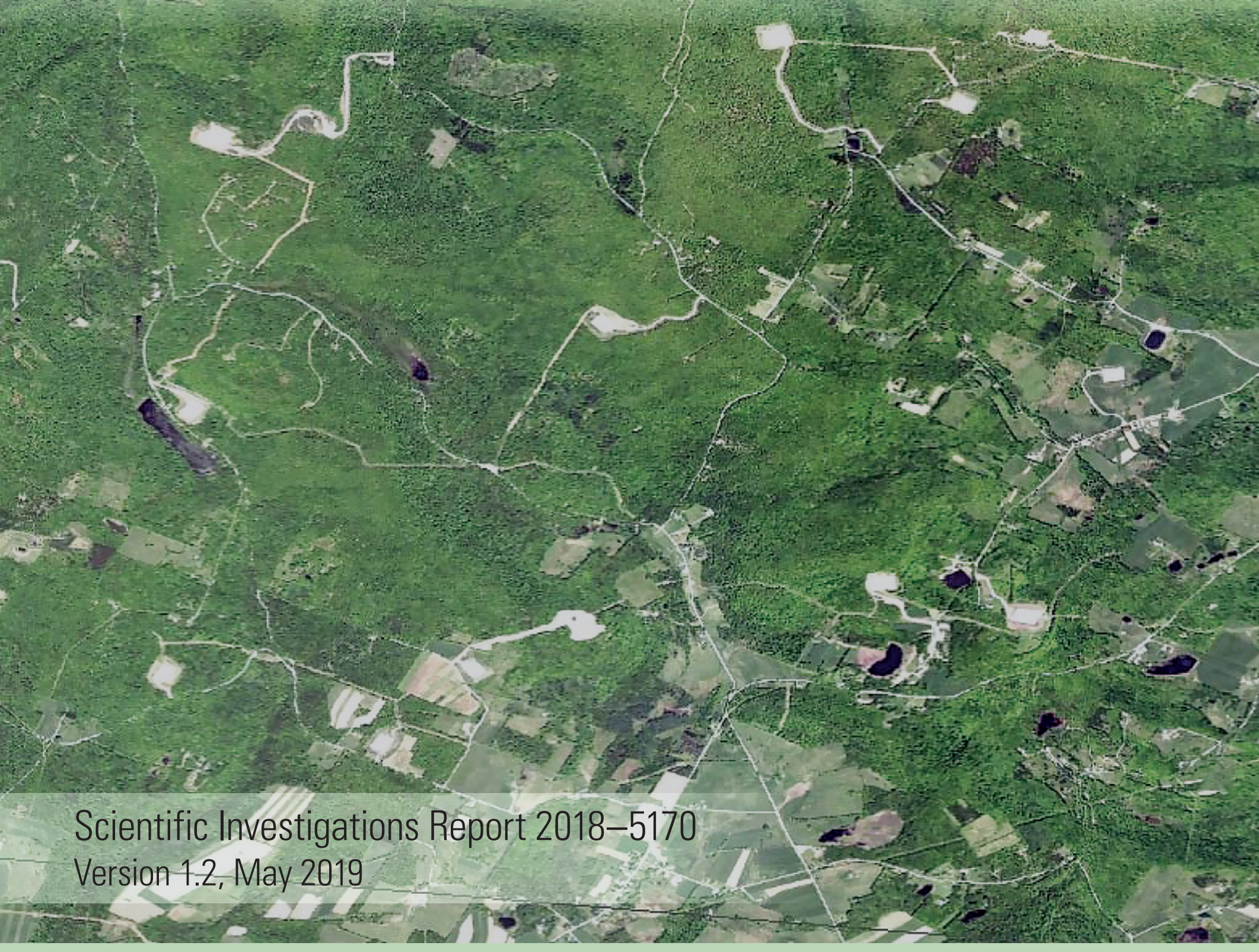

U.S. Department of the Interior U.S. Geological Survey 
Cover. The landscape of Bradford County, Pennsylvania, is predominately agricultural, forested areas, developed/residential land, and unconventional gas wells with related infrastructure. Aerial photograph by National Agriculture Imagery Program, 2016. 


\section{Drinking Water Health Standards Comparison and Chemical Analysis of Groundwater for 72 Domestic Wells in Bradford County, Pennsylvania, 2016}

By John W. Clune and Charles A. Cravotta III

Prepared in cooperation with the

Northern Tier Regional Planning and Development Commission

Scientific Investigations Report 2018-5170

Version 1.2, May 2019 


\title{
U.S. Department of the Interior \\ DAVID BERNHARDT, Acting Secretary
}

\author{
U.S. Geological Survey \\ James F. Reilly II, Director
}

\author{
U.S. Geological Survey, Reston, Virginia: 2019 \\ First release: 2019 \\ Revised: April 22, 2019 (ver. 1.1) \\ Revised: May 2019 (ver. 1.2)
}

\begin{abstract}
For more information on the USGS - the Federal source for science about the Earth, its natural and living resources, natural hazards, and the environment-visit https://www.usgs.gov or call 1-888-ASK-USGS.

For an overview of USGS information products, including maps, imagery, and publications,

visit https://store.usgs.gov.
\end{abstract}

\author{
Any use of trade, firm, or product names is for descriptive purposes only and does not imply endorsement by the \\ U.S. Government. \\ Although this information product, for the most part, is in the public domain, it also may contain copyrighted materials \\ as noted in the text. Permission to reproduce copyrighted items must be secured from the copyright owner. \\ Suggested citation: \\ Clune, J.W., and Cravotta, C.A., III, 2019, Drinking water health standards comparison and chemical analysis of \\ groundwater for 72 domestic wells in Bradford County, Pennsylvania, 2016 (ver 1.2, May 2019): U.S. Geological \\ Survey Scientific Investigations Report 2018-5170, 66 p., https://doi.org/10.3133/sir20185170. \\ ISSN 2328-0328 (online)
}




\section{Acknowledgments}

This project was funded by the Appalachian Regional Commission under a cooperative agreement with the Northern Tier Regional Planning Commission (NTRPC). The authors would like to thank Kimberly Barnes, Frank Thompson, Rachel Hauser, and Lauren Egleston with NTRPC, Matthew Williams with the Bradford County Planning Department, and Laura Fitzgerald with the Guthrie Clinic for making this study possible. Sincere appreciation is extended to all the county and township officials who helped with reconnaissance and location of eligible wells. Thanks are extended to U.S. Geological Survey colleagues for the groundwater sampling conducted by Lee Eicholtz, Dennis Low, Dana Heston, Matthew Conlon, Kyle Ohnstad, Frederick Fenner, and Kimberly Barry, and for report reviews performed by Tammy Zimmerman, Brandon Fleming, and William Kappel. The authors also sincerely appreciate the generosity of local landowners who made their wells accessible for sampling. 


\section{Contents}

Acknowledgments .......................................................................................................................ii

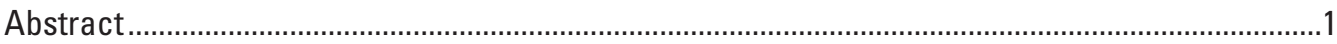

Introduction

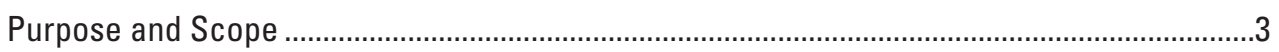

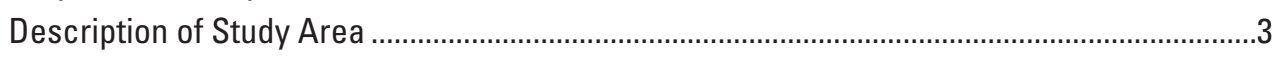

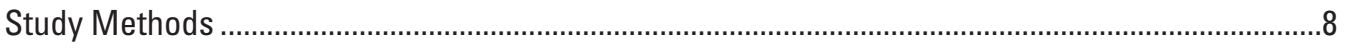

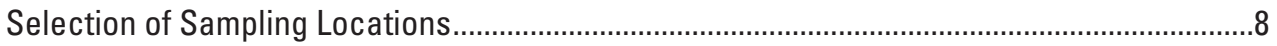

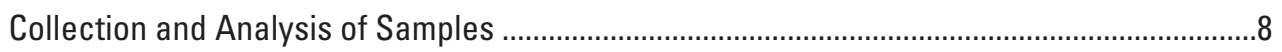

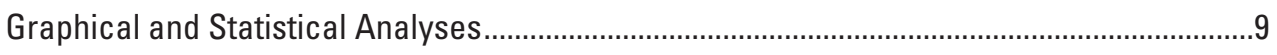

Quality Assurance and Quality Control ..............................................................................

Groundwater Quality and Comparison to Drinking Water Health Standards ..................................9

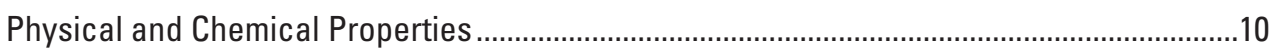

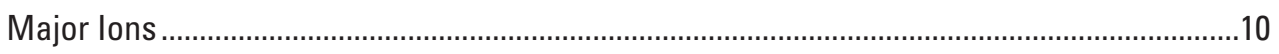

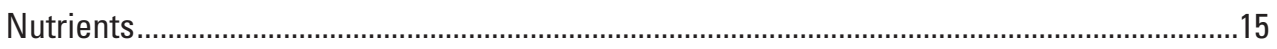

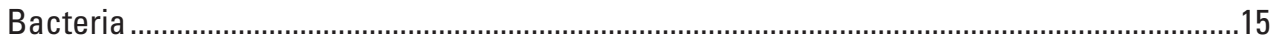

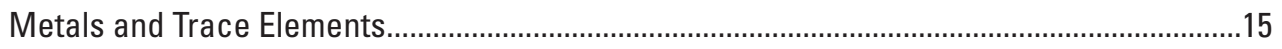

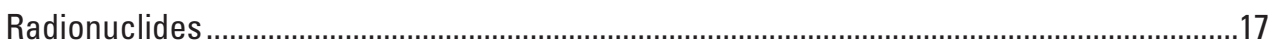

Dissolved Methane and Other Naturally Occurring Hydrocarbon Gases................................17

Man-Made Organic Compounds......................................................................................18

Chemical Analysis and Relations Among Constituents in Groundwater.......................................18

Conceptual Hydrogeochemical Model ..........................................................................18

Relations Among pH, Specific Conductance, and Constituent Concentrations .............18

Ionic Contributions to Conductivity and Total Dissolved Solids ....................................19

Correlations Among Major and Trace Constituents in Groundwater......................................23

Relations Between Groundwater Quality, Geology, and Topographic Setting ........................23

Major Ion Compositions Indic ated by Trilinear Diagrams.............................................23

Chloride, Bromide, and Sodium in Groundwater ........................................................24

Isotopic Composition, Origin, and Spatial Distribution of Methane ..................................29

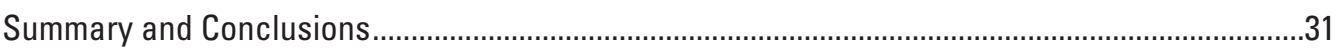

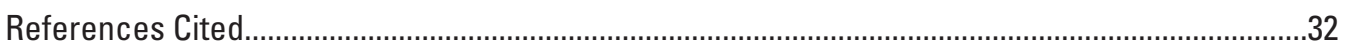

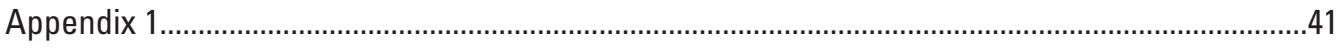

Appendix 2

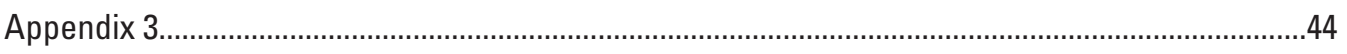

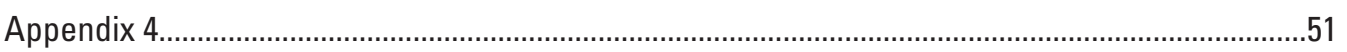




\section{Figures}

1. Maps showing $A$, bedrock geology, $B$, land use, and $C$, lithology and sampling sites in Bradford County, north-central Pennsylvania.

2. Graph showing groundwater levels for the period of record (1966-2016) for U.S. Geological Survey BR 92 Bradford County observation well (414330076280501).

3. Maps showing spatial distribution of $A$, arsenic concentrations, $B$, chloride concentrations, $C, \mathrm{pH}$ values, and $D$, methane concentrations in 72 wells sampled and bedrock geology in Bradford County, Pennsylvania

4. Graphs showing relation between $\mathrm{pH}$ and selected constituents for 72 groundwater samples in Bradford County, Pennsylvania.

5. Graphs showing concentrations of selected constituents in groundwater from Bradford County, Pennsylvania, 2016, compared to: $A-C, \mathrm{pH}$; or $D-F$, specific conductance.

6. Graphs showing major ion contributions to specific conductance (SC) for 72 groundwater samples from Bradford County, Pennsylvania, 2016:

$A$, comparison of estimated ionic conductivity contributions by sodium, calcium, magnesium, chloride, bicarbonate, and sulfate to measured SC for all 72 samples; and $B$, relative contributions by major ion species to computed $S C$ and selected groundwater samples of representative water types, expressed in percent, in order of increasing SC.

7. Pie charts showing water types and ionic contributions to specific conductance computed for selected groundwater samples from Bradford County, Pennsylvania, 2016

8. Trilinear diagrams showing $A$, predominant water types or hydrochemical facies; and $B$, data for 72 groundwater samples from Bradford County, Pennsylvania, 2016, plus median composition of brine from oil and gas wells in western Pennsylvania and flowback water from Marcellus Shale gas wells.

9. Graphs showing chloride concentrations compared to $A$, chloride/bromide mass ratios, $B$, bromide concentrations, and $C$, sodium contentrations for groundwater from Bradford County, Pennsylvania, 2016, plus median values for Salt Spring, flowback waters from Marcellus Shale gas wells, and oil and gas well brines from western Pennsylvania

10. Graphs showing $A$, isotopic composition of methane in groundwater samples collected from five wells in Bradford County, Pennsylvania, 2016; and B, ratio of methane to higher chain hydrocarbons $(\mathrm{C} 1 / \mathrm{C} 2+)$ in relation to carbon isotopic composition for methane in these samples

\section{Tables}

1. Minimum, median, and maximum values of selected characteristics and constituents in groundwater samples collected in 72 domestic wells in Bradford County, Pennsylvania, May-August 2016. 


\section{Conversion Factors}

U.S. customary units to International System of Units

\begin{tabular}{|c|c|c|}
\hline Multiply & By & To obtain \\
\hline \multicolumn{3}{|c|}{ Length } \\
\hline foot $(\mathrm{ft})$ & 0.3048 & meter $(\mathrm{m})$ \\
\hline mile (mi) & 1.609 & kilometer $(\mathrm{km})$ \\
\hline \multicolumn{3}{|c|}{ Area } \\
\hline square mile $\left(\mathrm{mi}^{2}\right)$ & 2.590 & square kilometer $\left(\mathrm{km}^{2}\right)$ \\
\hline \multicolumn{3}{|c|}{ Volume } \\
\hline gallon (gal) & 3.785 & liter $(\mathrm{L})$ \\
\hline \multicolumn{3}{|c|}{ Flow rate } \\
\hline gallon per minute (gal/min) & 0.06309 & liter per second $(\mathrm{L} / \mathrm{s})$ \\
\hline \multicolumn{3}{|c|}{ Pressure } \\
\hline inch of mercury at $60^{\circ} \mathrm{F}$ (in $\mathrm{Hg}$ ) & 3.377 & kilopascal (kPa) \\
\hline \multicolumn{3}{|c|}{ Radioactivity } \\
\hline picocurie per liter $(\mathrm{pCi} / \mathrm{L})$ & 0.037 & becquerel per liter $(\mathrm{Bq} / \mathrm{L})$ \\
\hline
\end{tabular}

Temperature in degrees Celsius $\left({ }^{\circ} \mathrm{C}\right)$ may be converted to degrees Fahrenheit $\left({ }^{\circ} \mathrm{F}\right)$ as follows:

$$
{ }^{\circ} \mathrm{F}=\left(1.8 \times{ }^{\circ} \mathrm{C}\right)+32
$$

Temperature in degrees Fahrenheit $\left({ }^{\circ} \mathrm{F}\right)$ may be converted to degrees Celsius $\left({ }^{\circ} \mathrm{C}\right)$ as follows:

$$
{ }^{\circ} \mathrm{C}=\left({ }^{\circ} \mathrm{F}-32\right) / 1.8
$$

\section{Datum}

Vertical coordinate information is referenced to the North American Vertical Datum of 1988 (NAVD 88).

Horizontal coordinate information is referenced to the North American Datum of 1983 (NAD 83). Altitude, as used in this report, refers to distance above the vertical datum.

\section{Supplemental Information}

Specific conductance is given in microsiemens per centimeter at 25 degrees Celsius $(\mu \mathrm{S} / \mathrm{cm}$ at $\left.25^{\circ} \mathrm{C}\right)$.

Concentrations of chemical constituents in water are given either in milligrams per liter (mg/L) or micrograms per liter $(\mu \mathrm{g} / \mathrm{L})$. 


\title{
Drinking Water Health Standards Comparison and Chemical Analysis of Groundwater for 72 Domestic Wells in Bradford County, Pennsylvania, 2016
}

\author{
By John W. Clune and Charles A. Cravotta III
}

\section{Abstract}

Pennsylvania has the second highest number of residential wells of any state in the Nation with approximately 2.4 million residents that depend on groundwater for their domestic water supply. Despite the widespread reliance on groundwater in rural areas of the state, publicly available data to characterize the quality of private well water are limited. In Bradford County, more than half of the residents use groundwater from private domestic-supply wells as their primary drinking source. The quality of private well water is influenced by the regional and local setting, including the surrounding soil, geology, land use, household plumbing, and well construction. The groundwater used for domestic water supply in Bradford County is obtained primarily from shallow bedrock and from unconsolidated (glacial) deposits that overlie the bedrock. Historical land use has been predominately forested, agricultural, and residential, but more recently unconventional oil/gas development has been distributed throughout the landscape. Pennsylvania is one of only two states in the Nation without statewide water-well construction standards.

To better assess the quality of groundwater used for drinking water supply in Bradford County, data for 72 domestic wells were collected and analyzed for a wide range of constituents that could be evaluated in relation to drinking water health standards, geology, land use, and other environmental factors. Groundwater samples were collected from May through August 2016 and analyzed for physical and chemical properties, including major ions, nutrients, trace elements, volatile organic compounds, ethylene and propylene glycol, alcohols, gross-alpha/beta-particle activity, uranium, radon-222, and dissolved gases. A subset of samples was analyzed for radium isotopes (radium-226 and -228) and for the isotopic composition of methane. This study was conducted by the U.S. Geological Survey in cooperation with the Northern Tier Regional Planning and Development Commission and is part of a regional effort to characterize groundwater in rural areas of Pennsylvania.

Results of the 2016 study show that groundwater quality generally met most drinking-water standards. However, a percentage of samples failed to meet maximum contaminant levels (MCLs) for total coliform bacteria (49.3 percent), Escherichia coli (8.5 percent), barium (2.8 percent), and arsenic (2.8 percent); and secondary maximum contaminant levels (SMCL) for sodium (48.6 percent), manganese (30.6 percent), gross alpha and beta activity (16.7 percent), iron (11.1 percent), $\mathrm{pH}$ (8.3 percent), total dissolved solids (5.6 percent), chloride (1.4 percent), and aluminum (1.4 percent). Radon-222 activities exceeded the proposed drinking-water standard of 300 picocuries per liter $(\mathrm{pCi} / \mathrm{L})$ in 70.4 percent of the samples. There were no exceedances of drinking water health standards for any volatile organic compounds, and the only detections were for three trihalomethanes in one sample.

The $\mathrm{pH}$ of the groundwater had a large influence on chemical characteristics and ranged from 6.18 to 9.31. Generally, the higher $\mathrm{pH}$ samples had higher potential for elevated concentrations of several constituents, including total dissolved solids, sodium, lithium, chloride, fluoride, boron, arsenic, and methane. For the Bradford County well-water samples, calcium/bicarbonate type waters were most abundant, with others classified as sodium/bicarbonate or mixed water types including calcium-sodium/bicarbonate, calcium-sodium/ bicarbonate-chloride, sodium/bicarbonate-chloride, sodium/ bicarbonate-sulfate, or sodium/chloride types. Six principal components ( $\mathrm{pH}$, redox, hardness, chloride-bromide, strontium-barium, and molybdenum-arsenic) explained nearly 78.3 percent of the variance in the groundwater dataset.

Groundwater from 12.5 percent of the wells had concentrations of methane greater than the Pennsylvania action level of 7 milligrams per liter $(\mathrm{mg} / \mathrm{L})$; detectable methane concentrations ranged from 0.01 to $77 \mathrm{mg} / \mathrm{L}$. In addition, low levels of ethane (as much as $0.13 \mathrm{mg} / \mathrm{L}$ ) were present in seven samples with the highest methane concentrations. The isotopic composition of methane in five of these groundwater samples was consistent with the isotopic compositions reported for mud-gas logging samples from these geologic units and a thermogenic source. Isotopic composition from a sixth sample suggested the methane in that sample may be of microbial origin. Well-water samples with the higher methane concentrations also had higher $\mathrm{pH}$ values and elevated concentrations of sodium, lithium, boron, fluoride, arsenic, and bromide. 
Relatively elevated concentrations of some other constituents, such as barium and chloride, commonly were present in, but not limited to, those well-water samples with elevated methane.

Four of the six groundwater samples with the highest methane concentrations had chloride/bromide ratios that indicate mixing with a small amount of brine $(0.02$ percent or less) similar in composition to those reported for gas and oil well brines in Pennsylvania. In several other eastern Pennsylvania counties where gas drilling is absent, groundwater with comparable chloride/bromide ratios and chloride concentrations have been reported, implying a potential natural source of brine. Most of Bradford County well-water samples have chloride concentrations less than $20 \mathrm{mg} / \mathrm{L}$, and those with higher chloride concentrations have chloride/bromide ratios that indicate anthropogenic sources (such as road-deicing salt and septic effluent) or brine. Brines that are naturally present may originate from deeper parts of the aquifer system, whereas anthropogenic sources are more likely to affect shallow groundwater because they occur on or near the land surface.

The available data for this study indicate that no one physical factor, such as the topographic setting, well depth, or altitude at the bottom of the well, was particularly useful for predicting those well locations with an elevated dissolved concentration of methane. The 2016 assessment of groundwater quality in Bradford County shows groundwater is generally of good quality, but methane and some constituents that occur in high concentration in naturally occurring brine and also in produced waters may be present at low to moderate concentrations in groundwater in various parts of the aquifer.

\section{Introduction}

Pennsylvania has the second highest number of residential wells of any state in the Nation with approximately 2.4 million residents that depend on groundwater for their domestic water supply. Despite the widespread reliance on groundwater in rural areas of the state, publicly available data to characterize the quality of private well water are limited (Low and Chichester, 2006; Giddings, 2014; Johnson and Belitz, 2017). In Bradford County, more than half of the residents use groundwater from private domestic-supply wells as their primary drinking source (Pennsylvania Bureau of Topographic and Geologic Survey, written commun., 2017).

Groundwater from more than one in five private domestic-supply wells in the Nation potentially exceeds a humanhealth standard and yet, the potential health risks associated with domestic groundwater supplies are often understudied in comparison to the research and testing of public water supplies (DeSimone, 2009). Unlike public water-supply systems, managing domestic-supply wells in Pennsylvania is solely the responsibility of the homeowner. Acute gastrointestinal illnesses have been attributed to parasitic, viral, and bacterial pathogens in rural private well water (Raina and others, 1999; Murphy and others, 2016). Metals such as lead can be present in the aquifer or leached from plumbing components by corrosive groundwater, causing delayed mental and physical development (Brown and Margolis, 2012; Belitz and others, 2016), and long-term exposure to arsenic has been linked to skin and internal cancers (National Research Council, 1999). The presence of metals like iron and manganese in drinking water can be a nuisance owing to the unpleasant taste, odor, and color and often require additional maintenance and treatment of household plumbing systems (Penn State Extension, 2017). The complex mixture and breakdown of radioactive elements and volatile organic compounds (VOCs) in drinking water also has been shown to be carcinogenic (Otton, 1992; National Research Council, 1998; Hopke and others, 2000). Elevated levels of dissolved gases like methane can pose an explosive safety risk if private wells are not properly vented (Swistock and Sharpe, 2006).

The quality of private well water is influenced by the regional and local setting, including the surrounding soil, geology, land use, and household plumbing. In addition, improper well construction (such as, no grouting and [or] sanitary well cap) can facilitate the introduction of potential contaminants from the surface into private groundwater wells and local aquifers (Zimmerman and others, 2001; Simpson, 2004; Swistock and Sharpe, 2005; Giddings, 2014). Despite legislative attempts, Pennsylvania is one of two states without statewide water-well construction standards (Swistock, 2012; Wagner , 2012; LaRegina, 2013; Giddings, 2014; Pennsylvania General Assembly, 2015a,b, 2016).

Previous national and statewide studies that include Bradford County provide general characterizations of water quality for a bedrock and glaciated groundwater system. In glaciated settings of the United States, the quality of groundwater is vulnerable to dissolved solids from the natural geology, anthropogenic contaminants such as rising chloride levels, and nuisance constituents like iron (Warner and Ayotte, 2015). Sampling of private wells in Pennsylvania show bedrock geology and well construction have a significant influence on water quality, and common contaminants often include bacteria, low $\mathrm{pH}$, arsenic, lead, nitrate, and radon (Swistock and others, 1993; Swistock and others, 2009).

Early regional groundwater assessments in the Bradford County area show comparatively moderate dissolved solids and hardness, with the most troublesome constituents being naturally occurring iron and manganese (Taylor and others, 1983). The predominant hydrogeochemical groundwater zones exhibit a sodium-chloride water type in the bedrock (restricted flow) and calcium/bicarbonate in the glaciated valleys (unrestricted flow) (Williams and others, 1998). Wells that have open holes that have been drilled in the restricted groundwater flow of bedrock formations can produce naturally elevated levels of chloride, barium, strontium, and radium (Williams and others, 1998; Yager and others, 2017).

More recent research that has furthered the understanding of local groundwater quality in Bradford County and 
surrounding counties has been a byproduct of the unconventional natural gas development in areas of Pennsylvania underlain by the Marcellus Shale since 2005 (U.S. Environmental Protection Agency, 2012b; Vidic and others, 2013; Soeder, 2017). Groundwater sampling from a subset of private domestic-supply wells before and after gas well drilling did not suggest major influences from gas well drilling, but did show that 40 percent of the samples failed at least one common drinking water health standard such as coliform bacteria (Boyer and others, 2012). Most well water chemistry in the region has been shown to be comparable to historical groundwater (American Petroleum Institute/America's Natural Gas Alliance, 2013) with some evidence of animal waste, septic effluent, or road salt but no detectable impact of flowback waters from gas well development (Reilly and others, 2015). Pre- and post-drilling comparisons show the quality of water from private domestic-supply wells is largely influenced by groundwater contact time (residence time) with the natural geology (Weston Solutions, 2012). Freshwater is often found where rainwater has more recently flushed the shallow aquifer, whereas brackish water may be encountered in deeper formations that have the remains of connate (water trapped in the pores of sedimentary sediments when deposited) seawater (Lohman, 1939). Analysis of pre-drilling groundwater shows the natural migration of Appalachian Basin brines along natural pathways - such as faults, fractures, lineaments, and so forth - in the bedrock of some shallow aquifers (Warner and others, 2012; Llewellyn, 2014). Analysis of robust propriety groundwater datasets from the natural gas industry show common exceedances of drinking water health standards for sodium, manganese, iron, lithium, turbidity, low $\mathrm{pH}$, arsenic, lead, and barium that are often related to the geological formation, water type, and topographic position (Siegel and others, 2015).

Naturally occurring thermogenic- and biogenic-derived dissolved hydrocarbon gases such as methane can also be common in groundwater from private domestic-supply wells (Molofsky and others, 2011, 2013; Heisig and Scott, 2013; Botner and others, 2018), although the potential for stray gas from natural gas extraction development is a concern (Osborn and others, 2011; Jackson and others, 2013; Heilweil and others, 2015; Llewellyn and others, 2015) especially in valleys and near faults (Wen and others, 2018).

The U.S. Geological Survey (USGS) has conducted a series of county groundwater studies since 2007 in order to provide publicly available data to better characterize the quality of groundwater in rural areas of Pennsylvania. Studies in Pike County and Sullivan County indicated few exceedances of the drinking water health standards, and samples that had elevated $\mathrm{pH}$ and methane often showed higher concentrations of sodium, lithium, boron, fluoride, and bromide (Senior, 2009, 2014; Sloto, 2013; Senior and Cravotta, 2017). Studies in Wayne County and Lycoming County presented similar results but also included bacteria sampling that indicated exceedances of the drinking water health standards and geochemical modeling that offered further insight into the effect that water-rock reactions, topographic position, and $\mathrm{pH}$ have on the regional groundwater chemistry (Senior and others, 2017; Gross and Cravotta, 2017).

The research described in this report is a continuation of these ongoing USGS county groundwater quality assessments in Pennsylvania characterizing the quality of groundwater in Bradford County and aims to further the national, statewide, and regional understanding of previous groundwater investigations. At the local level, the results will serve the needs of resource managers with a comparison of the quality of private well water with drinking water health standards and provide a conceptual model of the natural and anthropogenic influences on local groundwater quality. This study was conducted by the U.S. Geological Survey in cooperation with the Northern Tier Regional Planning and Development Commission and is part of a regional effort to characterize groundwater in rural areas of Pennsylvania.

\section{Purpose and Scope}

The purpose of this report is to (1) compare the groundwater quality from private domestic-supply wells to drinkingwater standards, and (2) to provide a chemical analysis of the natural and anthropogenic influences on groundwater quality in Bradford County, Pennsylvania. These objectives were met by the collection of analytical data for groundwater from 72 domestic wells sampled throughout Bradford County, Pennsylvania, from May through August of 2016. Groundwater samples were analyzed for physical and chemical properties, including major ions, nutrients, trace elements, VOCs, ethylene and propylene glycol, alcohols, gross-alpha/ beta-particle activity, uranium, radon-222, and dissolved gases. A subset of samples was analyzed for radium isotopes (radium-226 and -228) and for the isotopic composition of methane. The measured concentrations of analyzed constituents in groundwater were compared to U.S. Environmental Protection Agency (EPA) drinking-water standards. The groundwater-quality data, summary statistics, and geochemical computations are presented to further the understanding of the current (2016) chemical quality of groundwater from freshwater aquifers used by private domestic-supply wells in Bradford County. The relations among observed groundwaterquality characteristics, geology, topographic setting, land use, and other environmental variables associated with sampled wells are evaluated to explain the variability in the quality of the groundwater.

\section{Description of Study Area}

Bradford County occupies approximately 1,147 square miles in the glaciated portion of the Appalachian Plateaus Physiographic Province in north-central Pennsylvania (fig. 1A). The geology of Bradford County is characterized as gently folded clastic sedimentary bedrock that is overlain in most places by unconsolidated glacial deposits and alluvium 
(less than 20 feet thick). The bedrock is composed primarily of shale, siltstone, and sandstone strata of Devonian (400 million years ago) to Pennsylvanian (300 million years ago) age, including (from youngest to oldest) the Allegheny and Pottsville Formation, Burgoon Sandstone, Huntley Mountain Formation, Catskill Formation, and Lock Haven Formation (Miles and others, 2001). Fractures in the bedrock provide water-bearing zones that store and transfer groundwater to wells. In the valleys, layers of fluvial outwash and lacustrine (lake) deposits from past glaciations have filled the lowlands with mostly unconsolidated silt, clay, sand, and gravel (fig. 1C). Figure $1 C$ does not show lithology of surficial material or bedrock for the entire county which is often a mix of both. Private domestic-supply wells may be completed in bedrock or the overlying unconsolidated deposits. Wells drilled in bedrock typically are cased from the surface down to competent bedrock without grouting and consist of an open hole through the water-bearing zone, whereas those constructed in

A. Bedrock geology

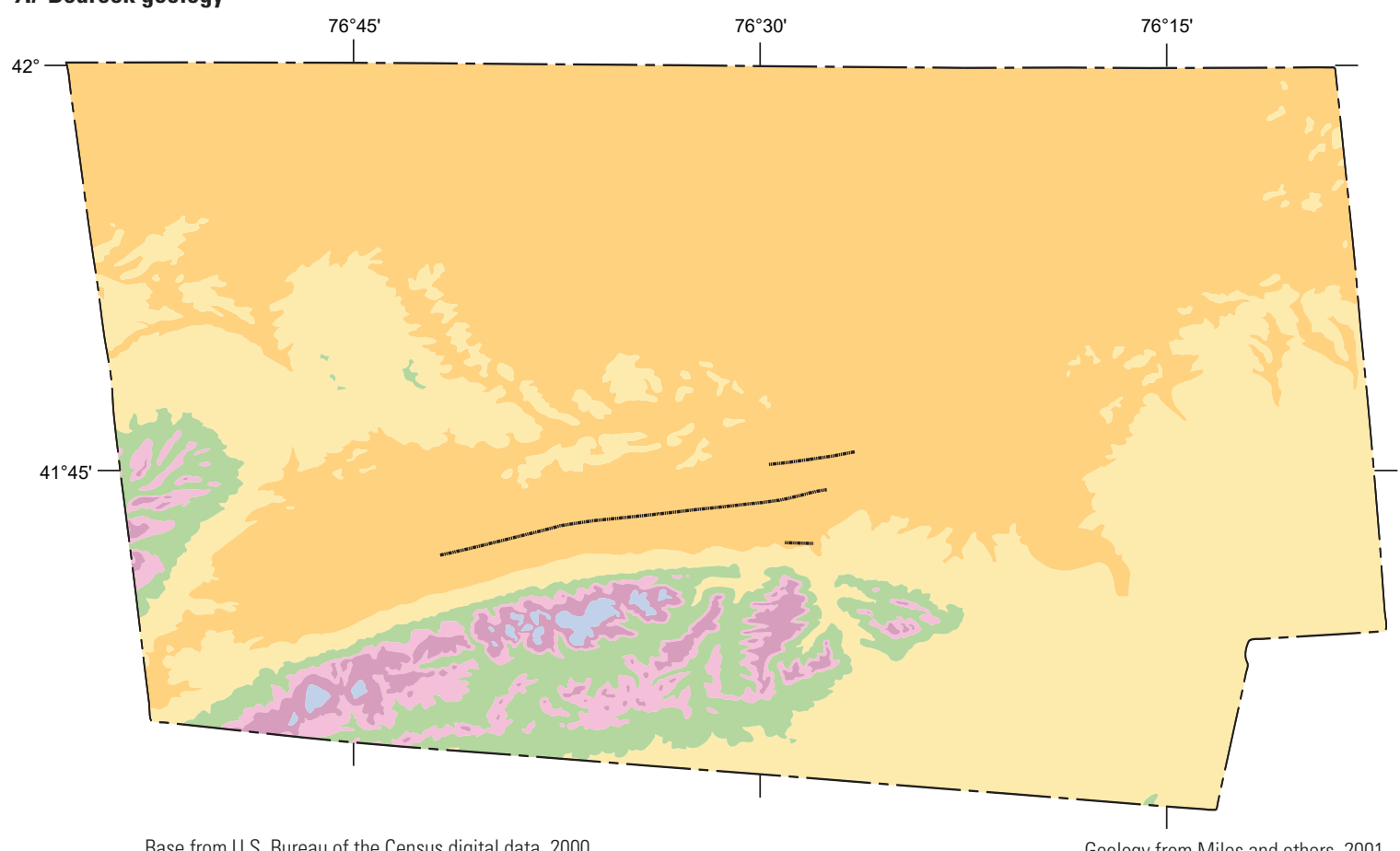

Base from U.S. Bureau of the Census digital data, 2000

Geology from Miles and others, 2001

Standard parallels $40^{\circ} \mathrm{N}$ and $42^{\circ} \mathrm{N}$, central meridian $-78^{\circ} \mathrm{W}$, latitude of origin $39^{\circ}$

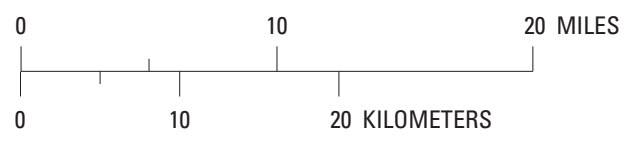

EXPLANATION

Allegheny and Pottsville Formations

$\begin{array}{lll}0 & 10 & 20 \text { KILOMETERS }\end{array}$

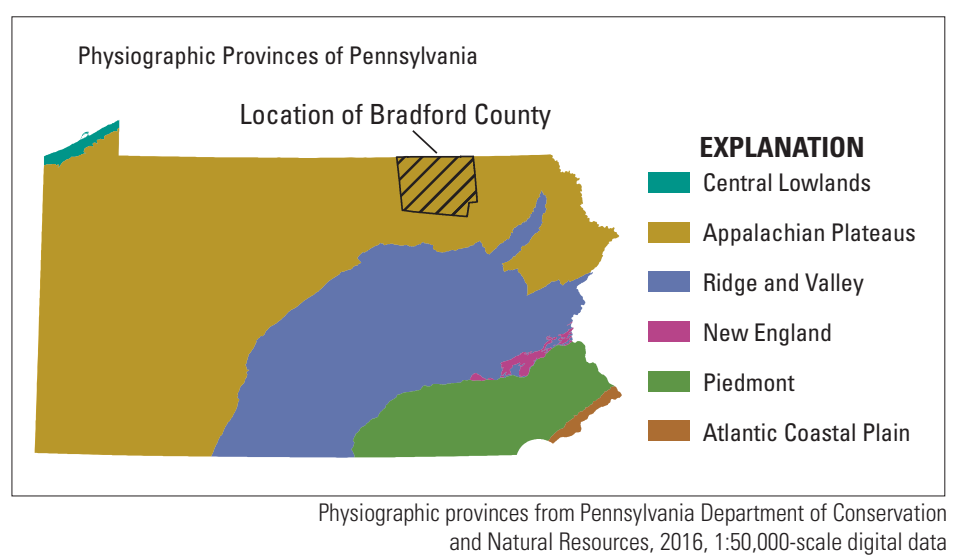

Figure 1. $A$, bedrock geology, $B$, land use, and $C$, lithology and sampling sites in Bradford County, north-central Pennsylvania. Bradford County is in a section of the glaciated region of the Appalachian Plateaus Physiographic Province. 
unconsolidated sand/gravel may be through open-ended casing or screened in the water-bearing zone.

Land use is predominately forested (56.1 percent), mostly on private land except for a small portion of Pennsylvania state forest, one state park, and several state game lands. Agricultural land use consists mainly of pasture/hay (23.5 percent) and cropland (11.4 percent; fig. 1B) (Multi-Resolution Land Characteristics Consortium, 2014). During the summer of 2016, Bradford County was second among all counties in the state for the most horizontal unconventional gas wells drilled ( $\mathrm{n}=1,339$; Pennsylvania Department of Environmental Protection, 2017), and the related infrastructure (well pads, pipelines, and so forth) has become a relatively recent part of the landscape. Wetland and water resources compose 2.67 percent and 0.98 percent of the landscape, respectively. The remaining land area is developed/residential land (4.95 percent) with a largely rural population of 62,622 recorded in 2010 (U.S. Census Bureau, 2011). Some of the most densely populated areas in the county include the boroughs of Sayre, Athens, and Towanda, and the townships of Athens, Canton,

\section{B. Land use}

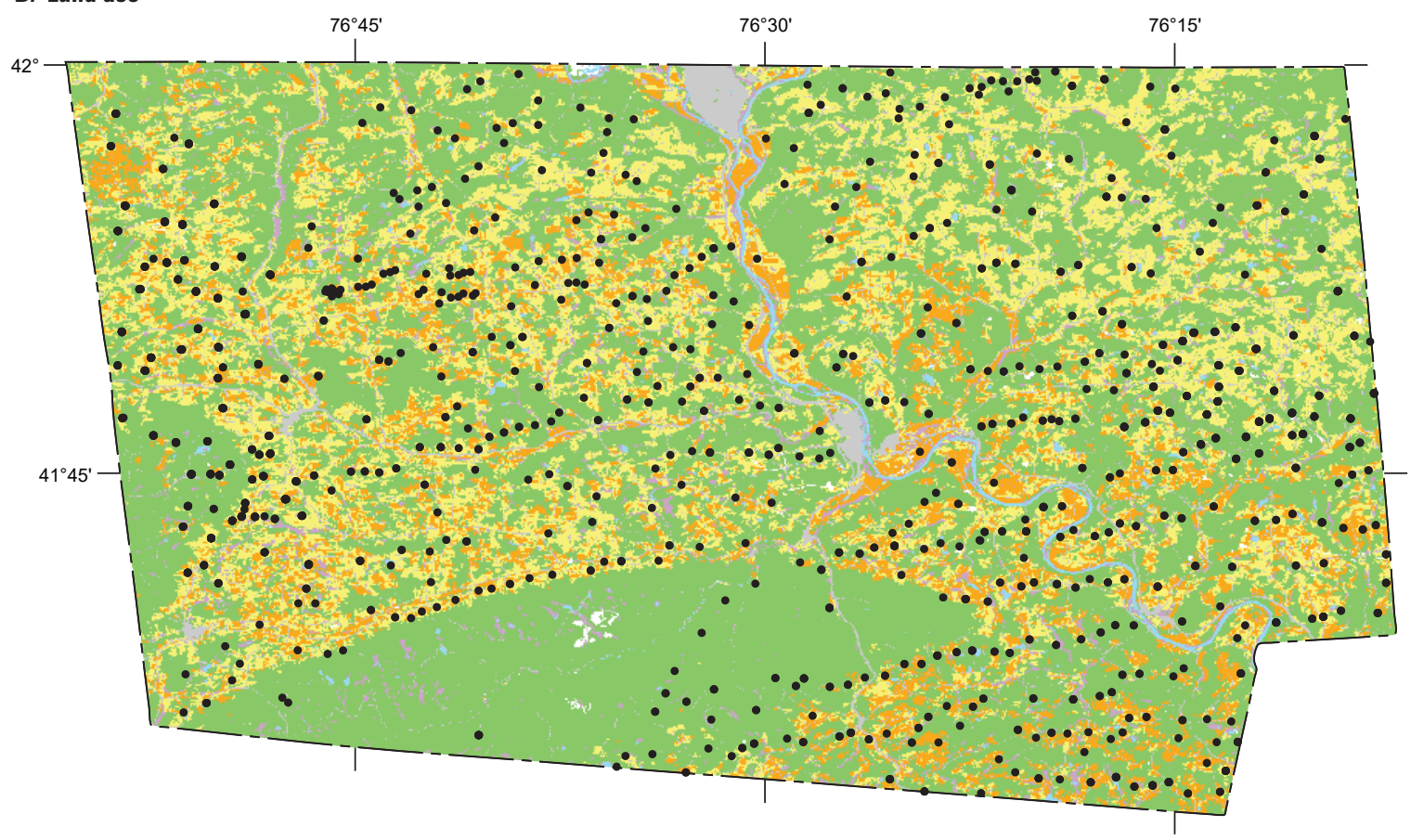

Base from U.S. Bureau of the Census digital data, 2000

1:100,000-scale Albers equal-area projection

Standard parallels $40^{\circ} \mathrm{N}$ and $42^{\circ} \mathrm{N}$, central meridian $-78^{\circ} \mathrm{W}$, latitude of origin $39^{\circ}$
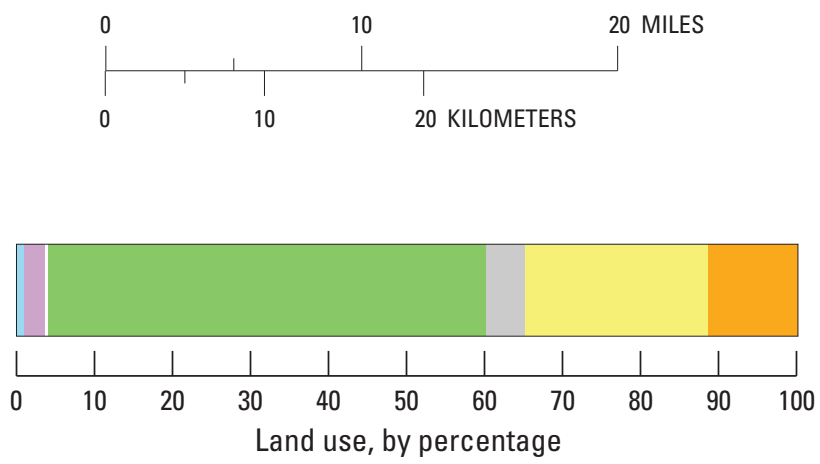

\section{EXPLANATION}

Land-use category (by percentage)—Land use from 2011 National Land Cover Dataset (NLCD), U.S. Geological Survey, 2014

Water (0.98)

Wetlands (2.67)

Barren land (0.36)

Forest (56.11)

Developed (4.95)

Pasture/hay (23.47)

Cropland (11.44)

- $\quad$ Natural gas well-Natural gas well locations from Pennsylvania Department of Environmental Protection, 2016

Figure 1. $A$, bedrock geology, $B$, land use, and $C$, lithology and sampling sites in Bradford County, north-central Pennsylvania. Bradford County is located in a section of the glaciated region of the Appalachian Plateaus Physiographic Province.-Continued 
and Ridgebury, with populations ranging from approximately 2,000 to 6,000 residents (U.S. Census Bureau, 2011).

Topography in Bradford County consists mostly of low to moderate relief with rounded hills and valleys with elevations ranging from $636 \mathrm{feet}(\mathrm{ft})$ to $2,710 \mathrm{ft}$ above sea level (Sevon, 2000; U.S. Geological Survey, 2009). The main streams within the county are Towanda, Sugar, Wysox, and Wyalusing Creeks that flow into the north branch of the Susquehanna River watershed, which eventually drains to the Chesapeake Bay. The local climate provides for a wet season of increased precipitation from April to June, with a mean annual rainfall of 37 inches per year (in/yr), of which approximately 10-12 inches recharges the aquifer (Risser and others, 2008). Groundwater levels fluctuate seasonally with increased recharge in the spring (March to May), a relatively stable water table during winter (November to February), and a steady decline during the summer (June to August) months owing to increased evapotranspiration (fig. 2). Average temperatures throughout the year in Bradford County range from about 33.6 to 55.6 degrees Fahrenheit (PRISM Group at Oregon State University, 2006a,b).

C. Lithology and sampling sites

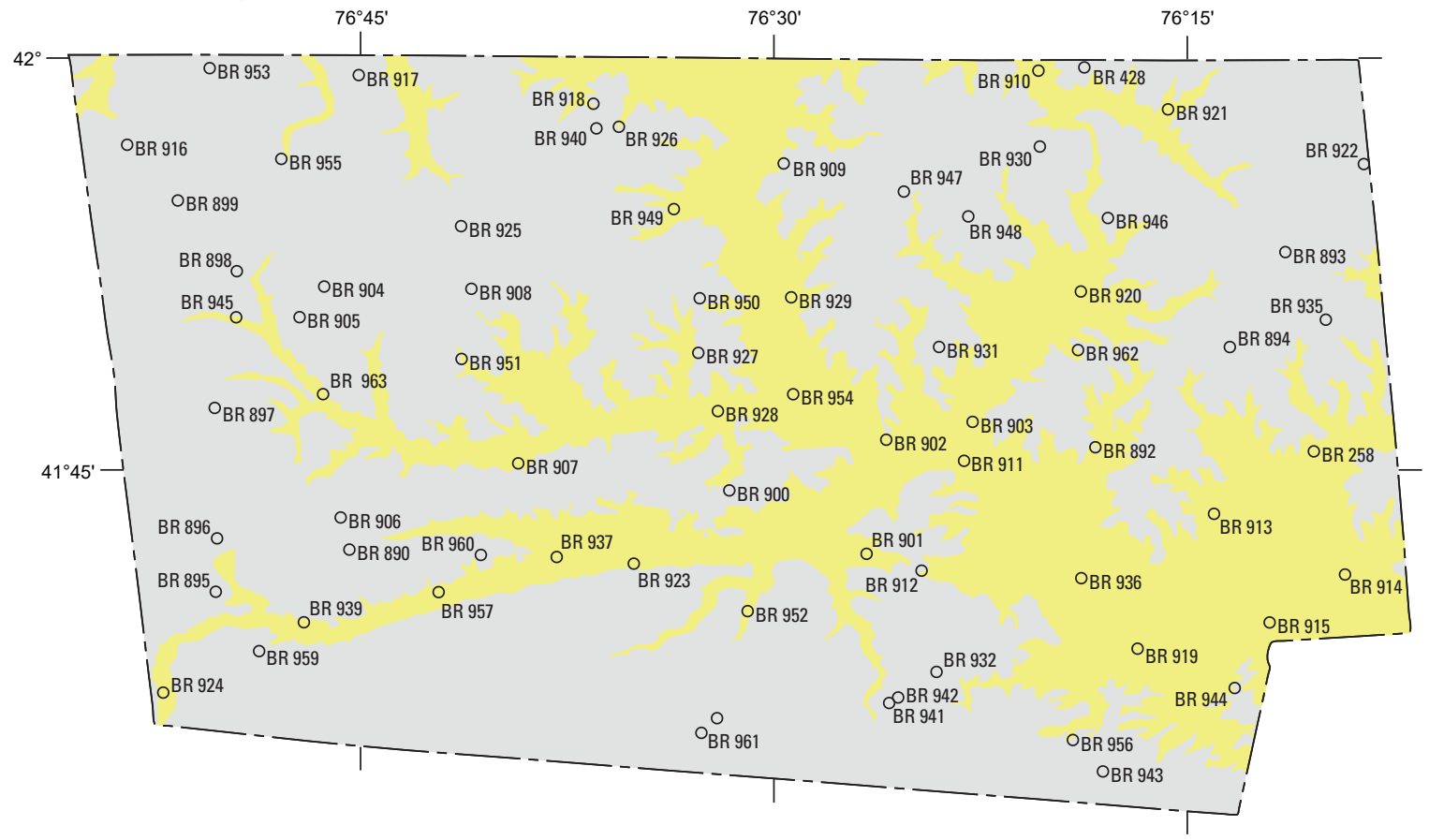

Base from U.S. Bureau of the Census digital data, 2000

1:100,000-scale Albers equal-area projection

Standard parallels $40^{\circ} \mathrm{N}$ and $42^{\circ} \mathrm{N}$, central meridian $-78^{\circ} \mathrm{W}$, latitude of origin $39^{\circ}$
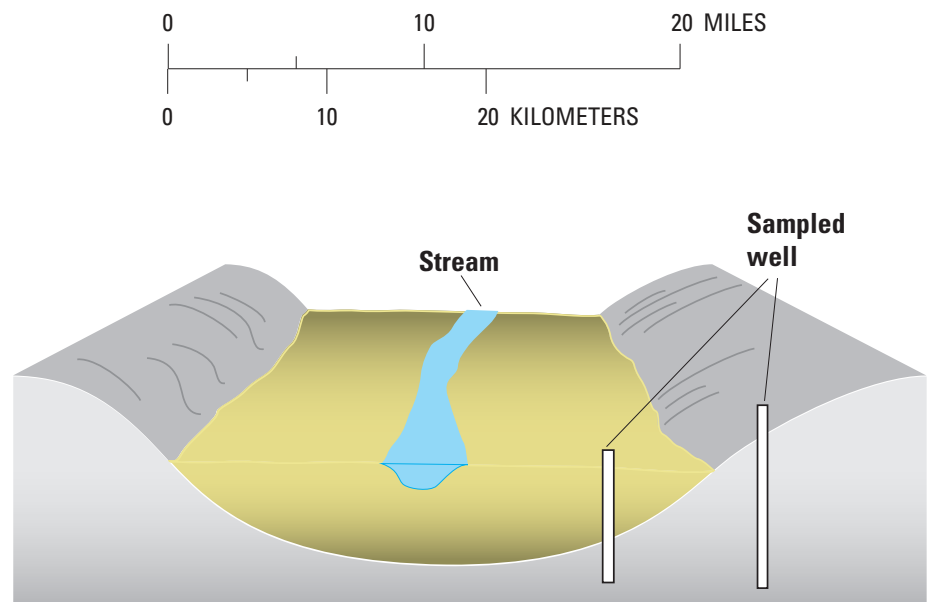

Not to scale

\section{Types of sampling locations}

Figure 1. A, bedrock geology, $B$, land use, and $C$, lithology and sampling sites in Bradford County, north-central Pennsylvania. Bradford County is located in a section of the glaciated region of the Appalachian Plateaus Physiographic Province.-Continued 


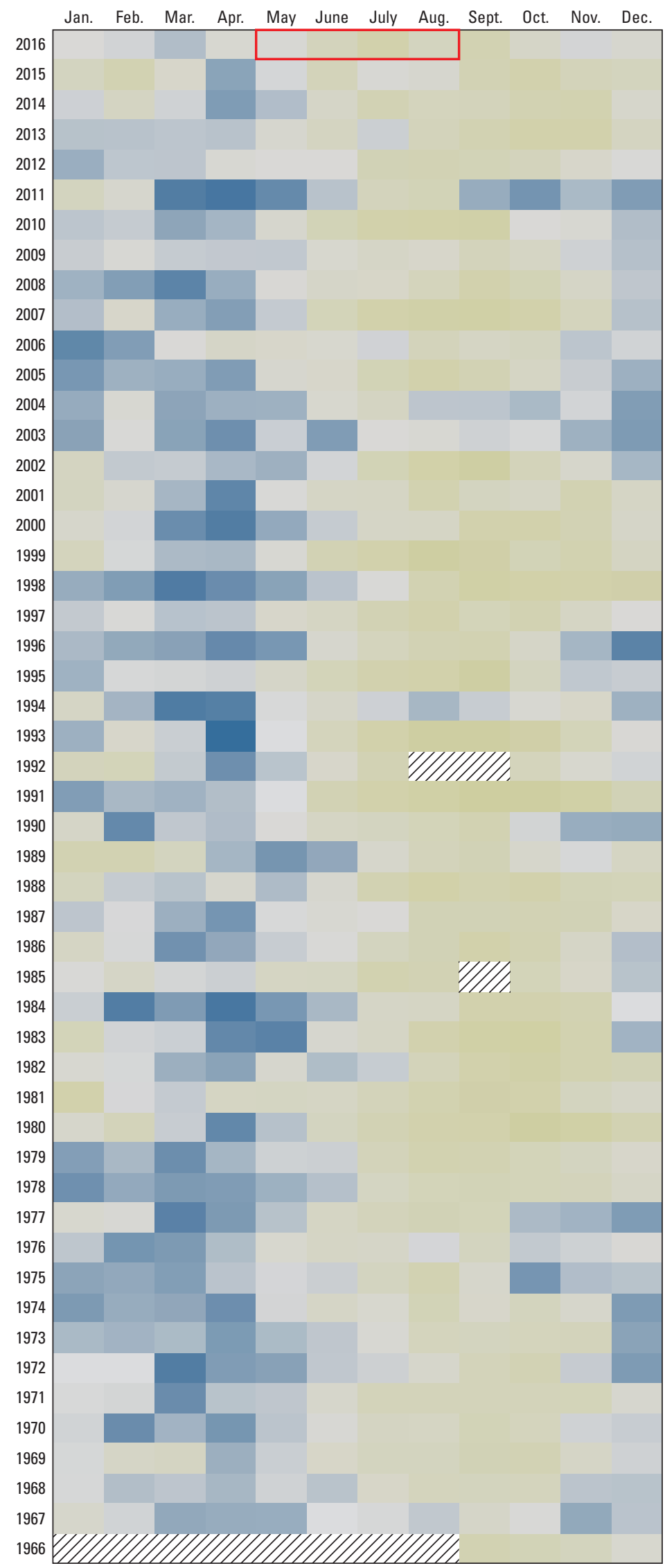

\section{EXPLANATION}

Groundwater levels - Water table

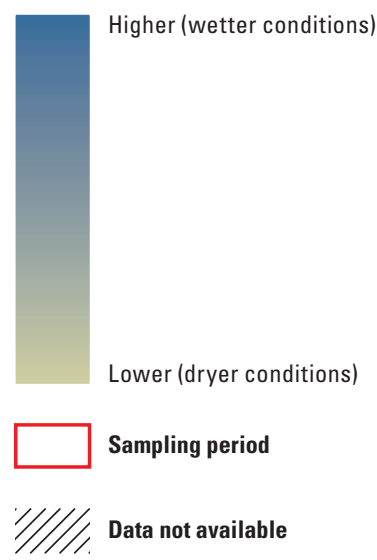

Figure 2. Groundwater levels for the period of record (1966-2016) for U.S. Geological Survey BR 92 Bradford County observation well (414330076280501). The water table in Bradford County fluctuates seasonally with increased precipitation/recharge (wetter conditions) in the spring (March to May) and declines during the summer (June to August) due to increased evapotranspiration (drier conditions). Groundwater samples were collected across Bradford County, Pennsylvania, from May to August 2016 (shown in red). 


\section{Study Methods}

To provide a characterization of groundwater quality in Bradford County, 72 private domestic-supply wells throughout the county (fig. 1) were selected for sampling during the summer months of 2016 (fig. 2). One sample from each well was analyzed for physical and chemical properties, including major ions, nutrients, trace elements, VOCs, ethylene and propylene glycol, gross-alpha/beta-particle activity, uranium, radon-222, and dissolved gases. A subset of samples was analyzed for radium isotopes (radium-226 and -228) and for the isotopic composition of methane. A list of wells sampled, ancillary data, and a National Water Information System (NWIS) website URL to water quality results used for analysis are provided by Clune and Cravotta (2018). Using the station identification numbers, the NWIS water quality results can also be retrieved with the dataRetrieval R package (Hirsh and DeCicco, 2015; Hirsh and others, 2015a, 2015b) or through user defined queries using USGS web services (U.S. Geological Survey, 2018). Additionally, quality assurance data results collected during sampling are available (Clune and Cravotta, 2018).

\section{Selection of Sampling Locations}

A computerized, stratified random site-selection approach was used to design a groundwater-quality network for sampling (Scott, 1990) by creating an equal-area grid of cells with a randomly placed population of potential sites across the county. The program arbitrarily specified one site from each cell as a primary sampling point and three additional sites as alternate sampling locations. The best available well record data within Bradford County were obtained from the Pennsylvania Groundwater Information System database (Pennsylvania Bureau of Topographic and Geologic Survey, 2014) and from local well drillers. Potential wells for sampling were selected from these data based on the criteria that the well was (1) used for domestic or stock purposes, (2) had an associated well drillers record, (3) was drilled after 1970, (4) was not hand dug, and (5) the top of the casing was above land surface. Wells located within 1 mile of one of the computer-generated random sampling points were identified, and well owners were contacted for permission to sample and to schedule a plumbing review to verify an untreated sample representative of the aquifer could be collected. Private domestic-supply wells meeting the criteria and well verification were prioritized for sampling.

\section{Collection and Analysis of Samples}

Sampling sites included 72 groundwater well locations throughout Bradford County (fig. 1C) drilled with an open hole interval usually in bedrock and sometimes unconsolidated deposits. Groundwater samples were collected from May through August 2016 (fig. 2) following protocols outlined in the USGS National Field Manual (U.S. Geological Survey, variously dated). Groundwater samples were collected using Teflon tubing attached to a sampling point (outside spigot, pressure tank, and so forth) prior to any water treatment. Wells were purged until field parameters $(\mathrm{pH}$, specific conductance, water temperature, turbidity, and dissolved oxygen) stabilized and the final readings were recorded. Samples were then collected for laboratory analysis.

The samples for total dissolved solids, major ion, trace metals, and nutrient chemistry were filtered onsite through a disposable filter with a 0.45 -micrometer pore size and analyzed by the National Water Quality Lab in Denver, Colorado. Samples for major ions were preserved with nitric acid if the $\mathrm{pH}$ was greater than 2 and analyzed at the lab by inductively coupled plasma atomic emission spectrometry, ion chromatography, or automated colorimetry (EPA Method 524). Nutrient analyses were performed by automated colorimetry with persulfate digestion when required (Fishman, 1993; Patton and Kryskalla, 2011).

Radiological samples for alpha/beta particles were preserved with nitric acid if $\mathrm{pH}$ was greater than 2 and analyzed at ALS Laboratories in Fort Collins, Colorado, by gas flow proportional counting (EPA Method 900.0). A subset of samples that had gross alpha greater than 5 picocuries per liter $(\mathrm{pCi} / \mathrm{L})$ were analyzed further for radium-226 and -228 by radon emanation, chemical separation, and gas flow proportional counting (EPA Method 903.1 and 904.0). Radon-222 samples were obtained through an inline septum with a gastight syringe to avoid atmospheric contact and analyzed at the lab by liquid scintillation (ASTM Method D 5072-16).

Dissolved gases sampling used a collection bag, and samples were analyzed at Isotech Laboratories, Champaign, Illinois, by gas compositional analysis to determine the concentration of fixed gases and light hydrocarbon gases dissolved in the sample (Dai and others, 2012). Samples containing a sufficient concentration of methane were further analyzed for stable carbon isotopes ${ }^{12} \mathrm{C}$ and ${ }^{13} \mathrm{C}$ and the stable hydrogen isotopes ${ }^{1} \mathrm{H}$ and ${ }^{2} \mathrm{H}$ (deuterium). The ${ }^{13} \mathrm{C} /{ }^{12} \mathrm{C}$ and ${ }^{2} \mathrm{H} /{ }^{1} \mathrm{H}$ ratios were determined by an isotope ratio mass spectrometric analysis that compared the sample to a reference standard. The carbon isotope ratio value in methane $\left(\delta^{13} \mathrm{C} \mathrm{CH}_{4}\right)$ is reported in terms of per mil (\%) notation with respect to the Vienna Peedee belemnite standard. The hydrogen isotope ratio value in methane $\left(\delta \mathrm{D} \mathrm{CH} \mathrm{CH}_{4}\right)$ is reported in terms of per mil notation with respect to the Vienna Standard Mean Ocean Water standard.

The remaining unfiltered samples were analyzed by Seewald Laboratories, Inc., in Williamsport, Pennsylvania. VOCs, dissolved gases, and glycol samples were collected onsite using a stainless-steel manifold fitting to fill vials to reduce turbulence. VOC vials were preserved with hydrochloric acid and analyzed by purge and trap gas chromatography/mass spectrometry (EPA Method 524.2). Dissolved gas samples for ethane and propane were determined by analyzing a portion of the headspace with a gas chromatograph equipped with a flame ionization detector (PA DEP Method 3686). Based upon 
the equilibrium gas concentration, a liquid sample concentration of these gases is calculated. Samples for glycols and alcohols were evaluated using the direct injection method, which uses a gas chromatograph equipped with a flame ionization detector that is chromatographically optimized for the separation and determination of the compounds of interest (EPA Method 8015 D). Oil and grease samples were preserved with hydrochloric acid and analyzed in the lab using hexane to determine the petroleum hydrocarbon fraction (EPA Method $1664 \mathrm{~B})$. After sterilizing and flaming the sampling point, the bacteria sample was collected and analyzed by the lab using the Colilert color method in which samples were enumerated to give counts of total coliform and Escherichia coli (Standard Method 9223).

\section{Graphical and Statistical Analyses}

Various graphical and statistical techniques were used in this report to compare water-quality data among different sites, to distinguish natural and anthropogenic sources of dissolved constituents, and to identify possible factors affecting the occurrence or transport of solutes in the aquifers in the study area. Techniques included bivariate scatter plots, mass ratios of chloride to bromide $(\mathrm{Cl} / \mathrm{Br})$, trilinear diagrams, nonparametric statistical approaches, correlation coefficients (Spearman's rho), principal components analysis (PCA), and aqueous speciation computations. The boxplots in the appendix considered pH (fig. 4.1), specific conductance (fig. 4.2), redox (fig. 4.3), bedrock formation (fig. 4.4), and topographic position index (fig. 4.5) to classify the data. The boxplots show a notched interval around the median that can be used by a reader to judge the significance of potential differences (Velleman and Hoaglin, 1981; Helsel and Hirsch, 2002). If the notched intervals around the medians for sample subsets do not overlap, the medians are statistically different at the 95-percent confidence interval. An extensive description of the graphical and statistical methods used in this report and the basis for classifications used in the appendix are described in Gross and Cravotta (2017).

\section{Quality Assurance and Quality Control}

For quality control (QC), filtered and unfiltered replicate samples were collected from wells BR 907, BR 910, BR 913, BR 939, and field blanks were processed onsite for BR 921, BR 923, and BR 940. All these samples were submitted to the laboratories for analysis. These results demonstrate that, for most constituents, field equipment or sampling methodology did not contaminate the sample. The results of the equipment blank indicate that sampling equipment may introduce low levels of two constituents into the samples at concentrations of as much as about 0.22 micrograms per liter $(\mu \mathrm{g} / \mathrm{L})$ for ammonia and $0.05 \mu \mathrm{g} / \mathrm{L}$ for molybdenum. The results of one of the three field blanks (BR 921) indicate that sampling methodology may introduce low levels of four constituents into samples at concentrations of as much as about 0.05 milligrams per liter ( $\mathrm{mg} / \mathrm{L})$ for calcium, $0.10 \mathrm{mg} / \mathrm{L}$ for sodium, $0.04 \mathrm{mg} / \mathrm{L}$ for chloride, and $0.03 \mathrm{mg} / \mathrm{L}$ for silica.

Results from three sets of replicate samples indicate combined sampling and analytical precision (reproducibility) was within 5 percent and (or) concentrations were less than two times the minimum reporting level for most constituents. Within two times the minimum reporting level, the concentrations were less precise but still within 20 percent, except for gross alpha and beta radioactivity. For the replicate sample collected from BR 939, calcium values were 77.3 and $66.6 \mathrm{mg} / \mathrm{L}$ with a difference of $10.7 \mathrm{mg} / \mathrm{L}$ ( 15 percent), and methane had low detected values of 0.0042 and $0.0037 \mathrm{mg} / \mathrm{L}$ with a difference of $0.0005 \mathrm{mg} / \mathrm{L}$ (13 percent). For the replicate sample collected from BR 910, bromide was 0.440 and $0.493 \mathrm{mg} / \mathrm{L}$ with a difference of $0.053 \mathrm{mg} / \mathrm{L}$ (11 percent difference). For the replicate sample at BR 907, ammonia was 0.29 and $0.33 \mathrm{mg} / \mathrm{L}$ with a difference of 0.04 (14 percent), and radon 222 was 324 and $349 \mathrm{pCi} / \mathrm{L}$ with a $25-\mathrm{pCi} / \mathrm{L}$ difference ( 7 percent). The two other replicate samples with values for calcium, methane, bromide, ammonia, and radon 222 had a less than 3-percent difference or were less than two times the minimum reporting level.

For quality assurance (QA) of the inorganic chemical analyses, intrasample characteristics were evaluated using standard procedures described by Hem (1985) and Fishman and Friedman (1989). Evaluations of accuracy and precision included comparison of field- and laboratory-measured values for $\mathrm{pH}$ and specific conductance for each sample. Additional QA/QC checks involved comparisons of the computed cation and anion equivalents concentrations and the corresponding ionic charge balance, comparisons of the ratios of cation or anion equivalents to specific conductance, and comparisons of total dissolved solids (TDS) computed as the sum of major ion concentrations to the measured residue on evaporation (ROE) at 180 degrees Celsius $\left({ }^{\circ} \mathrm{C}\right)$. Lastly, the measured specific conductance was compared to the computed specific conductance, which was estimated as the sum of ionic conductivities after accounting for aqueous speciation (McCleskey and others, 2012). The values of specific conductance measured in the field and laboratory were consistent with one another and with the computed values of specific conductance and TDS on the basis of measured solute concentrations. The measured TDS concentration as ROE by two different laboratories and the computed TDS as the sum of constituent concentrations were in close agreement.

\section{Groundwater Quality and Comparison to Drinking Water Health Standards}

Analytical results for the 72 groundwater samples collected in Bradford County are provided in the following sections and compared to EPA drinking-water standards and health advisories (U.S. Environmental Protection Agency, 
2012a). The EPA has established maximum contaminant levels (MCLs), secondary maximum contaminant levels (SMCLs), and action levels for some constituents in drinking water (table 1). MCLs generally are set because elevated concentrations of these constituents may cause adverse health effects. SMCLs generally are set for aesthetic reasons; elevated concentrations of these constituents may impart an undesirable taste or odor to water. These MCLs and SMCLs may be used as a guideline for private well owners but are only regulated for public drinking-water supplies.

\section{Physical and Chemical Properties}

Physical and chemical properties discussed in this section include temperature, $\mathrm{pH}$, specific conductance, dissolved oxygen, and turbidity. Most of these properties are unstable and are determined in the field at the time a water sample is collected.

The temperature of the groundwater samples ranged from 9.4 to $18.0^{\circ} \mathrm{C}$, with a median of $11.5^{\circ} \mathrm{C}$. These temperatures generally were less than the daytime air temperatures during sampling, which reflects the generally cool groundwater environment. Dissolved gases and carbonate minerals can dissolve to a greater extent in cooler water than in warmer water (Hem, 1985).

Dissolved oxygen (DO) concentrations ranged from 0.03 to $10.6 \mathrm{mg} / \mathrm{L}$; the median concentration was $1.21 \mathrm{mg} / \mathrm{L}$ (table 1). Generally, most of the groundwater had DO concentrations that were substantially less than saturation at the sample temperature, indicating the waters had been out of contact with the atmosphere. Low DO concentrations are associated with higher $\mathrm{pH}$ values; 21 of the 30 water samples with a DO concentration of less than $1 \mathrm{mg} / \mathrm{L}$ had a $\mathrm{pH}$ greater than 7.5. Low DO concentrations are related to chemical or biochemical reactions that consume oxygen and may be associated with chemically reducing conditions that promote the release of iron, manganese, and associated metals from rock and other solids in contact with water along its flow path. The chemical reactions that consume oxygen generally involve organic carbon that can be naturally occurring in soil or aquifer materials, or can be introduced from industrial, agricultural, or domestic wastes. Of the 72 well samples in this study, 36.1 percent were classified as "anoxic" (DO $<0.5 \mathrm{mg} / \mathrm{L}), 50.0$ percent as "oxic" (DO $\geq 0.5 \mathrm{mg} / \mathrm{L})$, and 13.9 percent as "mixed" (DO $>0.5 \mathrm{mg} / \mathrm{L}$ with manganese $(\mathrm{Mn})>0.05 \mathrm{mg} / \mathrm{L}$ or iron $(\mathrm{Fe})>0.1 \mathrm{mg} / \mathrm{L})$, based on criteria of McMahon and Chapelle (2008) (fig. 4.3). Of the 26 samples classified as anoxic, 12 had chemical characteristics consistent with MnIV and FeIII reducing conditions as defined by McMahon and Chapelle (2008) $(\mathrm{DO}<0.5 \mathrm{mg} / \mathrm{L}$, nitrate $\left(\mathrm{NO}_{3}\right)<0.5 \mathrm{mg} / \mathrm{L}, \mathrm{Mn}>0.05 \mathrm{mg} / \mathrm{L}, \mathrm{Fe}>0.1 \mathrm{mg} / \mathrm{L}$, and sulfate $\left.\left(\mathrm{SO}_{4}\right)>0.5 \mathrm{mg} / \mathrm{L}\right)$, and two were identified with more strongly reducing conditions necessary for methanogenesis $\left(\mathrm{DO}<0.5 \mathrm{mg} / \mathrm{L} ; \mathrm{NO}_{3}<0.5 \mathrm{mg} / \mathrm{L}, \mathrm{Mn}>0.05 \mathrm{mg} / \mathrm{L}, \mathrm{Fe}>0.1 \mathrm{mg} / \mathrm{L}\right.$, and $\left.\mathrm{SO}_{4}<0.5 \mathrm{mg} / \mathrm{L}\right)$. Both samples identified as methanogenic had elevated concentrations of methane; BR 910 and
BR 921 had methane concentrations of $77 \mathrm{mg} / \mathrm{L}$ and $26 \mathrm{mg} / \mathrm{L}$, respectively.

The $\mathrm{pH}$ is a measure of acidity and is related to the potential corrosivity of the water and its potential to leach metals, such as lead and copper, from pipes and plumbing. The standard $\mathrm{pH}$ scale is $0-14$, with 7 being neutral. Generally, $\mathrm{pH}$ values of 6.5 to 7.5 are considered near neutral, values less than 6.5 are considered acidic, and values greater than 7.5 are considered basic or alkaline. The field $\mathrm{pH}$ of water samples collected in Bradford County ranged from 6.18 to 9.31; the median $\mathrm{pH}$ was 7.47 . The $\mathrm{pH}$ of 6 of the 72 samples $(8.3$ percent) was outside the EPA SMCL range of 6.5-8.5 (U.S. Environmental Protection Agency, 2012a). Two samples had a $\mathrm{pH}$ less than 6.5 , and four samples had a $\mathrm{pH}$ greater than 8.5 (table 1 and fig. $3 C$ ). Generally, the $\mathrm{pH}$ varied widely within and among the three bedrock aquifers sampled; median $\mathrm{pH}$ values were greatest for the Lock Haven Formation (7.6), least for Huntley Mountain Formation (6.8), and intermediate for the Catskill Formation (7.4) (fig. 4.4).

Specific conductance (SC) is a gross measure of the ability of ions in water to conduct an electrical current and is expressed in units of microsiemens per centimeter $(\mu \mathrm{S} / \mathrm{cm})$. The higher the value for specific conductance, the higher the concentrations of total dissolved solids and associated ions in the water. The field-measured SC for the 72 groundwater samples ranged from 72.4 to $3,410 \mu \mathrm{S} / \mathrm{cm}$ at $25{ }^{\circ} \mathrm{C}$; the median specific conductance was $365 \mu \mathrm{S} / \mathrm{cm}$ (table 1). Values were similar for the laboratory-measured SC (table 1), which is useful because the laboratory measured SC avoids potential issues with air bubbles forming on the electrode surfaces when the sample is first drawn from the subsurface.

Turbidity is a measure of the amount of solid particles suspended in water that block the transmission of light through the sample. Turbidity is expressed in nephelometric turbidity ratio units (NTRU), which quantify the degree to which light is scattered by solid particles suspended in water. The higher the NTRU, the more turbid the water. Turbidity concentrations ranged from 0.12 to $247 \mathrm{NTRU}$; the median concentration was 1.19 NTRU (table 1). In general, samples with high turbidity could be expected to have total concentrations of constituents that include contributions from suspended particles, in addition to the dissolved concentration.

\section{Major lons}

Major ions are frequently derived from the dissolution of common minerals, including carbonates, silicates, oxides, sulfates, and sulfides, and can be influenced by ion-exchange, redox processes, and mixing of freshwater with residual brines that remain in the aquifer matrix or that could be mobilized from deep sources. The concentrations of major ions, TDS, salinity, and SC of groundwater are directly related, and generally expected to increase with, progressive evaporation or dissolution of minerals (Hem, 1985). The concentrations of trace elements in solution may increase with TDS or SC, not 
only because of the release of trace constituents with the major ions dissolved from minerals, but because of the potential for displacement of adsorbed or exchangeable trace ions from mineral surfaces by the major ions.

Major cations (positively charged ions such as calcium, magnesium, sodium, and potassium), major anions (negatively charged ions such as sulfate, chloride, fluoride, and bicarbonate), and nonionic solutes (uncharged solutes such as silica) typically are present in natural waters at concentrations greater than $1 \mathrm{mg} / \mathrm{L}$, whereas dissolved trace constituents (such as iron, manganese, zinc, lead, copper, nickel, vanadium, molybdenum, arsenic, selenium, radium, uranium, lithium, and bromide) typically are present at concentrations less than $1 \mathrm{mg} / \mathrm{L}$ (Hem, 1985). Concentrations of DO and nutrients (such as nitrogen and phosphorus compounds) commonly range from values less than $1 \mathrm{mg} / \mathrm{L}$ to values greater than $1 \mathrm{mg} / \mathrm{L}$ because of biological processes that involve the production or metabolism of organic carbon compounds that contain nitrogen and phosphorus. Although biological (biochemical) processes can affect the concentrations of nutrients and trace constituents in groundwater directly or indirectly because of changes to $\mathrm{pH}$ and redox, such processes generally have minor effects on major ion concentrations.

Analyses for major ions were conducted on filtered samples to represent dissolved concentrations. Major anions, in order of decreasing median dissolved concentration, were bicarbonate (alkalinity), sulfate, chloride, fluoride, and bromide; major cations were calcium, sodium, magnesium, and potassium. These cations and anions, plus uncharged silica, are the primary contributors to TDS and SC. Of the major ions, only fluoride has an established EPA MCL in drinking water $(4 \mathrm{mg} / \mathrm{L})$ or SMCL $(2 \mathrm{mg} / \mathrm{L})$. Fluoride concentrations ranged from 0.03 to $0.78 \mathrm{mg} / \mathrm{L}$, and none of the samples exceeded the EPA MCL or SMCL (table 1).

The concentration of TDS can be measured by evaporating a given volume of water and weighing the ROE or by summing the measured concentrations of the major ions in a filtered sample (TDScalc). The measured TDS concentrations ranged from 52.7 to $2,030 \mathrm{mg} / \mathrm{L}$; the median concentration was $218 \mathrm{mg} / \mathrm{L}$. The TDScalc was comparable in value to the $\mathrm{ROE}$ and ranged from 42.0 to $1,940 \mathrm{mg} / \mathrm{L}$. Out of 72 samples, 4 samples (5.6 percent) had TDS (ROE and TDScalc) that exceeded $500 \mathrm{mg} / \mathrm{L}$, which is the EPA SMCL for TDS in drinking water. One of these four samples had elevated concentrations of sulfate (BR 946) that may result from the oxidation of pyrite, whereas three samples (BR 913, BR 921, and BR 927) had elevated concentrations of sodium and chloride. Elevated sodium and chloride concentrations could be caused by connate water in the formations and a zone of restricted groundwater flow that limits flushing by fresh recharge; mixing of saline and freshwater; or by the introduction of mineral salts from near-surface contaminant sources such as road deicing compounds, water softener additives, effluent from septic systems, or animal waste (Mullaney and others, 2009).

Dissolved concentrations of sodium ranged from 2.18 to $760 \mathrm{mg} / \mathrm{L}$; the median concentration was $19.1 \mathrm{mg} / \mathrm{L}$.
The EPA has issued a taste threshold for sodium in public water supplies that recommends reducing sodium concentrations to between 30 and $60 \mathrm{mg} / \mathrm{L}$ (U.S. Environmental Protection Agency, 2003). This recommendation is a guideline and is not federally enforceable. Salt used for food, road deicing, and other applications is mainly composed of sodium chloride. Thus, high concentrations of sodium and chloride may result from the addition of salt to groundwater and may be related to land use as well as the bedrock and (or) unconsolidated lithology. Dissolved concentrations of chloride ranged from 0.77 to $1,020 \mathrm{mg} / \mathrm{L}$; the median concentration was $5.28 \mathrm{mg} / \mathrm{L}$ (table 1 , fig. $3 B$ ). Out of the 72 samples, only 1 sample (1.4 percent), BR 913, exceeded the EPA SMCL of $250 \mathrm{mg} / \mathrm{L}$ for chloride in drinking water.

The alkalinity of a solution indicates its capacity to neutralize acid and commonly results from dissolved carbonate and bicarbonate ions (Hem, 1985). Alkalinity, also referred to as the acid neutralizing capacity, generally increases with the $\mathrm{pH}$ of a water sample. Alkalinity ranged from 26.1 to $347 \mathrm{mg} / \mathrm{L}$ as calcium carbonate $\left(\mathrm{CaCO}_{3}\right)$; the median concentration was $178 \mathrm{mg} / \mathrm{L}$ (table 1). The highest alkalinities were from the Lock Haven Formation, and the lowest were from Huntley Mountain Formation (fig. 4.4).

The hardness of the 72 well-water samples ranged from 1.32 to $627 \mathrm{mg} / \mathrm{L}$ as $\mathrm{CaCO}_{3}$, with a median value of $134 \mathrm{mg} / \mathrm{L}$ as $\mathrm{CaCO}_{3}$. Hardness is directly related to the concentrations of calcium and magnesium (computed as the sum of calcium, in $\mathrm{mg} / \mathrm{L}$, multiplied by a factor of 2.5 plus magnesium, in $\mathrm{mg} / \mathrm{L}$, multiplied by a factor of 4.1) and generally is comparable to the alkalinity. The alkalinity and associated cations can be released into groundwater by the dissolution of calciumand magnesium-bearing minerals, such as calcite $\left(\mathrm{CaCO}_{3}\right)$ and dolomite $\left(\mathrm{CaMg}\left(\mathrm{CO}_{3}\right)_{2}\right)$, that generally are associated with limestone and other calcareous sedimentary rocks. Hard water decreases the lathering of soap and increases accumulation of mineral deposits in plumbing and cooking utensils. Using a common hardness classification (Durfor and Becker, 1964), the measured values indicate that 8 (11.1 percent) of the 72 water samples are soft (less than $60 \mathrm{mg} / \mathrm{L}$ as $\left.\mathrm{CaCO}_{3}\right), 19$ samples (26.4 percent) are moderately hard (61 to $120 \mathrm{mg} / \mathrm{L}$ as $\mathrm{CaCO}_{3}$ ), 27 samples (37.5 percent) are hard (121 to $180 \mathrm{mg} / \mathrm{L}$ as $\mathrm{CaCO}_{3}$ ), and 18 samples (25.0 percent) are classified as very hard (greater than $180 \mathrm{mg} / \mathrm{L}$ as $\mathrm{CaCO}_{3}$ ). Median hardness values were 52.1, 128, and $147 \mathrm{mg} / \mathrm{L}$ as $\mathrm{CaCO}_{3}$ for the Huntley Mountain, Catskill, and Lock Haven Formations, respectively. Hardness varied with $\mathrm{pH}$, with hardness generally greatest at near-neutral $\mathrm{pH}$ values (6.5 to 7.5). Water samples with low $\mathrm{pH}$ (less than 6.5) and very high $\mathrm{pH}$ (greater than 8.0 ) typically had hardness less than $60 \mathrm{mg} / \mathrm{L}$ and were considered soft. The high-pH waters with low hardness typically have elevated sodium, which may result from natural water-softening processes, which are described later. There are no health-related standards established specifically for hardness in drinking water.

Hard water has greater potential than soft water to form scale or encrustations on plumbing and fixtures. Water 
Table 1. Minimum, median, and maximum values of selected characteristics and constituents in groundwater samples collected in 72 domestic wells in Bradford County, Pennsylvania, May-August 2016. Available U.S. Environmental Protection Agency Maximum Contaminant Levels, Action Levels, and Secondary Maximum Contaminant Levels for analyzed constituents (2012 Edition of the Drinking Water Standards and Health Advisories [EPA 822-S-12-001])

[n, number of results; EPA, U.S. Environmental Protection Agency; MCL, Maximum Contaminant Level; SMCL, Secondary Maximum Contaminant Level; —, no MCL, Action Level, or SMCL established; ${ }^{\circ} \mathrm{C}$, degrees Celsius; $\mathrm{mg} / \mathrm{L}$, milligrams per liter; $\mu \mathrm{S} / \mathrm{cm}$, microsiemens per centimeter; $\mathrm{mV}$, millivolts; $\mathrm{CaCO}_{3}$, calcium carbonate; $\mathrm{SiO}_{2}$, silicon dioxide; $\mu \mathrm{g} / \mathrm{L}$, micrograms per liter; <, less than; MPN/100 mL, most probable number per 100 millimeters; >, greater than; $\mathrm{pCi} / \mathrm{L}$, picocuries per liter; 72 -hr, sample analyzed for gross alpha concentration at approximately 72 hours after sample collection as referenced to a detector calibrated using thorium-230 $\left({ }^{230} \mathrm{Th}\right) ; 30$-d, sample used for the 72 -hour gross alpha analysis is counted a second time approximately 30 days after the initial count as referenced to a detector calibrated using ${ }^{230} \mathrm{Th} ; \delta$, delta; mil, million]

\begin{tabular}{|c|c|c|c|c|c|c|c|c|c|c|c|}
\hline \multirow[t]{2}{*}{ Constituent (units) } & \multirow[t]{2}{*}{$\mathbf{n}$} & \multirow{2}{*}{ Minimum } & \multirow{2}{*}{ Median } & \multirow{2}{*}{ Maximum } & \multicolumn{2}{|c|}{$\begin{array}{l}\text { Results above the } \\
\text { reporting level }\end{array}$} & \multicolumn{3}{|c|}{ Results exceeding standard } & \multirow{2}{*}{$\begin{array}{l}\text { EPA } \\
\text { MCL }\end{array}$} & \multirow{2}{*}{$\begin{array}{l}\text { EPA } \\
\text { SMCL }\end{array}$} \\
\hline & & & & & Number & Percent & Number & Percent & Graph & & \\
\hline \multicolumn{12}{|c|}{ Well characteristics } \\
\hline Well depth (feet) & 72 & 42 & 183 & 600 & - & - & - & - & - & - & - \\
\hline \multicolumn{12}{|c|}{ Physical properties } \\
\hline Water temperature $\left({ }^{\circ} \mathrm{C}\right)$ & 72 & 9.40 & 11.5 & 18.0 & 72 & 100 & - & - & - & - & - \\
\hline Dissolved oxygen (mg/L) & 72 & 0.03 & 1.21 & 10.6 & 72 & 100 & - & - & - & - & - \\
\hline Specific conductance, field $(\mu \mathrm{S} / \mathrm{cm})$ & 72 & 72.4 & 365 & 3,410 & 72 & 100 & - & - & - & - & - \\
\hline pH, field (standard units) & 72 & 6.18 & 7.47 & 9.31 & 72 & 100 & 6 & 8.3 & 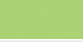 & - & $6.5-8.5$ \\
\hline Redox potential (mV) & 71 & -190 & 140 & 370 & 71 & 100 & - & - & - & - & - \\
\hline Turbidity (Nephelometric Turbidity Ratio Units) & 70 & 0.12 & 1.19 & 247 & 70 & 100 & - & - & - & - & - \\
\hline \multicolumn{12}{|c|}{ Laboratory analysis } \\
\hline Specific conductance, lab $(\mu \mathrm{S} / \mathrm{cm})$ & 72 & 79.3 & 369 & 3,800 & 72 & 100 & - & - & - & - & - \\
\hline pH, lab (standard units) & 72 & 6.96 & 8.09 & 9.16 & 72 & 100 & 3 & 4.2 & ta & - & $6.5-8.5$ \\
\hline Alkalinity, lab (mg/L CaCO$\left.)_{3}\right)$ & 72 & 26.1 & 178 & 347 & 72 & 100 & - & - & - & - & - \\
\hline Total dissolved solids (mg/L) & 72 & 52.7 & 218 & 2,030 & 72 & 100 & 4 & 5.6 & 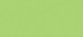 & - & 500 \\
\hline Suspended solids (mg/L) & 72 & 15.0 & 15.0 & 488 & 5 & 6.94 & - & - & - & - & - \\
\hline Hardness, total (mg/L CaCO $\left.{ }_{3}\right)$ & 71 & 1.32 & 134 & 627 & 71 & 100 & - & - & - & - & - \\
\hline \multicolumn{12}{|c|}{ Major ions } \\
\hline Calcium (mg/L) & 72 & 0.04 & 39.6 & 164 & 72 & 100 & - & - & - & - & - \\
\hline Magnesium (mg/L) & 72 & 0.01 & 8.37 & 51.6 & 72 & 100 & - & - & - & - & - \\
\hline Sodium (mg/L) & 72 & 2.18 & 19.1 & 760 & 72 & 100 & 35 & 48.6 & 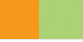 & - & $30-60$ \\
\hline Potassium (mg/L) & 72 & 0.22 & 1.40 & 4.89 & 72 & 100 & - & - & - & - & - \\
\hline Chloride (mg/L) & 72 & 0.77 & 5.28 & 1,020 & 72 & 100 & 1 & 1.4 & 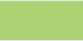 & - & 250 \\
\hline Fluoride (mg/L) & 72 & 0.03 & 0.12 & 0.78 & 71 & 98.6 & 0 & 0 & 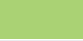 & 4 & 2 \\
\hline Sulfate $(\mathrm{mg} / \mathrm{L})$ & 72 & 0.06 & 13.5 & 418 & 71 & 98.6 & 0 & 0 & 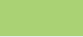 & - & 250 \\
\hline Silica $\left(\mathrm{mg} / \mathrm{L}\right.$ as $\left.\mathrm{SiO}_{2}\right)$ & 72 & 6.05 & 11.1 & 15.9 & 72 & 100 & - & - & - & - & - \\
\hline \multicolumn{12}{|c|}{ Trace elements } \\
\hline Aluminum $(\mu \mathrm{g} / \mathrm{L})$ & 72 & $<3.00$ & $<3.00$ & 771 & 10 & 13.9 & 1 & 1.4 & & - & 50 \\
\hline Antimony $(\mu \mathrm{g} / \mathrm{L})$ & 72 & $<0.02$ & $<0.02$ & 0.30 & 31 & 43.1 & 0 & 0 & & 6 & - \\
\hline Arsenic $(\mu \mathrm{g} / \mathrm{L})$ & 72 & $<0.05$ & 0.63 & 23.2 & 67 & 93.1 & 2 & 2.8 & & 10 & - \\
\hline Barium $(\mu \mathrm{g} / \mathrm{L})$ & 72 & $<0.25$ & 142 & 4,160 & 71 & 98.6 & 2 & 2.8 & - & 2,000 & - \\
\hline Beryllium ( $\mu \mathrm{g} / \mathrm{L})$ & 72 & $<0.01$ & 0.02 & 0.10 & 8 & 11.1 & 0 & 0 & & 4 & - \\
\hline Boron $(\mu \mathrm{g} / \mathrm{L})$ & 72 & $<5.00$ & 68.9 & 567 & 70 & 97.2 & - & - & - & - & - \\
\hline Bromide (mg/L) & 72 & $<0.01$ & 0.02 & 8.60 & 58 & 80.6 & - & - & - & - & - \\
\hline Cadmium $(\mu \mathrm{g} / \mathrm{L})$ & 72 & $<0.03$ & 0.03 & 0.31 & 2 & 2.78 & 0 & 0 & & 5 & - \\
\hline Chromium $(\mu \mathrm{g} / \mathrm{L})$ & 72 & $<0.30$ & $<0.30$ & 5.00 & 2 & 2.78 & 0 & 0 & . & 100 & - \\
\hline Cobalt $(\mu \mathrm{g} / \mathrm{L})$ & 72 & $<0.03$ & 0.05 & 0.75 & 17 & 23.6 & - & - & - & - & - \\
\hline Copper $(\mu \mathrm{g} / \mathrm{L})$ & 72 & $<0.20$ & 0.82 & 82.3 & 37 & 51.4 & 0 & 0 & . & - & 1,000 \\
\hline Iron $(\mu \mathrm{g} / \mathrm{L})$ & 72 & $<4.00$ & 11.34 & 1,830 & 44 & 61.1 & 8 & 11.1 & - & - & 300 \\
\hline Lead $(\mu \mathrm{g} / \mathrm{L})$ & 72 & $<0.02$ & 0.10 & 2.61 & 53 & 73.6 & 0 & 0 & . & 15 & - \\
\hline Lithium $(\mu \mathrm{g} / \mathrm{L})$ & 72 & 0.41 & 21.8 & 1,510 & 72 & 100 & - & - & - & - & - \\
\hline Manganese $(\mu \mathrm{g} / \mathrm{L})$ & 72 & $<0.40$ & 10.5 & 1,120 & 56 & 77.8 & 22 & 30.6 & 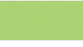 & - & 50 \\
\hline Molybdenum $(\mu \mathrm{g} / \mathrm{L})$ & 72 & $<0.05$ & 0.48 & 2.42 & 68 & 94.4 & - & - & - & - & - \\
\hline Nickel $(\mu \mathrm{g} / \mathrm{L})$ & 72 & $<0.20$ & $<0.20$ & 2.00 & 30 & 41.7 & - & - & - & - & - \\
\hline
\end{tabular}


Table 1. Minimum, median, and maximum values of selected characteristics and constituents in groundwater samples collected in 72 domestic wells in Bradford County, Pennsylvania, May-August 2016. - Continued Available U.S. Environmental Protection Agency Maximum Contaminant Levels, Action Levels, and Secondary Maximum Contaminant Levels for analyzed constituents (2012 Edition of the Drinking Water Standards and Health Advisories [EPA 822-S-12-001])

\begin{tabular}{|c|c|c|c|c|c|c|c|c|c|c|c|}
\hline \multirow[t]{2}{*}{ Constituent (units) } & \multirow[t]{2}{*}{$\mathbf{n}$} & \multirow[t]{2}{*}{ Minimum } & \multirow[t]{2}{*}{ Median } & \multirow[t]{2}{*}{ Maximum } & \multicolumn{2}{|c|}{$\begin{array}{l}\text { Results above the } \\
\text { reporting level }\end{array}$} & \multicolumn{3}{|c|}{ Results exceeding standard } & \multirow{2}{*}{$\begin{array}{l}\text { EPA } \\
\text { MCL }\end{array}$} & \multirow{2}{*}{$\begin{array}{l}\text { EPA } \\
\text { SMCL }\end{array}$} \\
\hline & & & & & Number & Percent & Number & Percent & Graph & & \\
\hline \multicolumn{12}{|c|}{ Trace elements-Continued } \\
\hline Selenium $(\mu \mathrm{g} / \mathrm{L})$ & 72 & $<0.05$ & $<0.05$ & 0.56 & 30 & 41.7 & 0 & 0 & & 50 & - \\
\hline Silver $(\mu \mathrm{g} / \mathrm{L})$ & 72 & $<0.02$ & $<0.02$ & 10.0 & 1 & 1.39 & 0 & 0 & & - & 100 \\
\hline Strontium $(\mu \mathrm{g} / \mathrm{L})$ & 72 & $<0.80$ & 539 & 3,860 & 71 & 98.6 & - & - & - & - & - \\
\hline $\operatorname{Zinc}(\mu \mathrm{g} / \mathrm{L})$ & 72 & $<2.00$ & 3.23 & 981 & 47 & 65.3 & 0 & 0 & & - & 5,000 \\
\hline \multicolumn{12}{|c|}{ Nutrients } \\
\hline Kjeldahl nitrogen, total ( $\mathrm{mg} / \mathrm{L}$ as Nitrogen) & 72 & $<0.07$ & 0.07 & 1.71 & 33 & 45.8 & - & - & - & - & - \\
\hline Ammonia (mg/L as Nitrogen) & 72 & $<0.01$ & $<0.01$ & 1.60 & 42 & 58.3 & - & - & - & - & - \\
\hline Nitrite ( $\mathrm{mg} / \mathrm{L}$ as Nitrogen) & 72 & $<0.001$ & $<0.001$ & 0.23 & 17 & 23.6 & 0 & 0 & 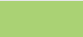 & 1 & - \\
\hline Nitrate + Nitrite $(\mathrm{mg} / \mathrm{L}$ as Nitrogen $)$ & 72 & $<0.04$ & $<0.04$ & 7.01 & 36 & 50.0 & 0 & 0 & . & 10 & - \\
\hline Orthophosphate (mg/L as Phosphorus) & 72 & $<0.004$ & 0.01 & 0.16 & 63 & 87.5 & - & - & - & - & - \\
\hline \multicolumn{12}{|c|}{ Bacteria } \\
\hline Total coliform (MPN/100 mL) & 71 & $<1$ & $<1$ & $>2,420$ & 35 & 49.3 & 35 & 49.3 & 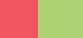 & 0 & - \\
\hline Escherichia Coli (MPN/100 mL) & 71 & $<1$ & $<1$ & $>2,420$ & 6 & 8.5 & 6 & 8.5 & & 0 & - \\
\hline \multicolumn{12}{|c|}{ Radiochemicals } \\
\hline Gross alpha radioactivity, $30-\mathrm{d}(\mathrm{pCi} / \mathrm{L})$ & 72 & -0.89 & 1.00 & 8.3 & 39 & 54.2 & 0 & 0.0 & & 15 & - \\
\hline Gross alpha radioactivity, $72-\mathrm{hr}(\mathrm{pCi} / \mathrm{L})$ & 72 & -0.33 & 1.70 & 11.2 & 52 & 72.2 & 0 & 0.0 & & 15 & - \\
\hline Gross beta radioactivity, $30-\mathrm{d}(\mathrm{pCi} / \mathrm{L})$ & 72 & -0.50 & 2.34 & 8.1 & 57 & 79.2 & 12 & 16.7 & & 4 & - \\
\hline Gross beta radioactivity, $72-\mathrm{hr}(\mathrm{pCi} / \mathrm{L})$ & 72 & 0.03 & 2.40 & 5.89 & 64 & 88.9 & 12 & 16.7 & & 4 & - \\
\hline Radon-222 (pCi/L) & 71 & 34.4 & 614 & 8,540 & 71 & 100 & 50 & 70.4 & & ${ }^{1} 300$ & - \\
\hline Uranium (pCi/L) & 72 & $<0.01$ & 0.26 & 10.4 & 62 & 86.1 & 0 & 0.0 & & 30 & - \\
\hline Radium-226 (pCi/L) & 8 & 0.16 & 1.08 & 2.08 & 8 & 100 & 0 & 0.0 & & 5 & - \\
\hline Radium-228 (pCi/L) & 8 & -0.02 & 0.31 & 1.54 & 4 & 50.0 & 0 & 0.0 & & 5 & - \\
\hline \multicolumn{12}{|c|}{ Glycols, alcohols and oil/grease } \\
\hline Ethanol $(\mathrm{mg} / \mathrm{L})$ & 72 & $<5.00$ & $<5.00$ & $<5.00$ & 0 & 0 & - & - & - & - & - \\
\hline Ethylene glycol (mg/L) & 72 & $<5.00$ & $<5.00$ & $<5.00$ & 0 & 0 & 0 & 0.0 & 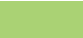 & 14 & - \\
\hline Isobutyl alcohol (mg/L) & 72 & $<5.00$ & $<5.00$ & $<5.00$ & 0 & 0 & - & - & - & - & - \\
\hline Isopropyl alcohol (mg/L) & 72 & $<5.00$ & $<5.00$ & $<5.00$ & 0 & 0 & - & - & - & - & - \\
\hline Methanol (mg/L) & 72 & $<5.00$ & $<5.00$ & $<5.00$ & 0 & 0 & - & - & - & - & - \\
\hline$n$-Butanol (mg/L) & 72 & $<5.00$ & $<5.00$ & $<5.00$ & 0 & 0 & - & - & - & - & - \\
\hline$n$-Propanol (1-Propanol) (mg/L) & 72 & $<5.00$ & $<5.00$ & $<5.00$ & 0 & 0 & - & - & - & - & - \\
\hline Propylene glycol (mg/L) & 72 & $<5.00$ & $<5.00$ & $<5.00$ & 0 & 0 & - & - & - & - & - \\
\hline Oil and grease $(\mathrm{mg} / \mathrm{L})$ & 72 & $<5.00$ & $<5.40$ & $<6.00$ & 0 & 0 & - & - & - & - & - \\
\hline \multicolumn{12}{|c|}{ Volatile organic compounds ${ }^{2}$} \\
\hline Bromodichloromethane $(\mathrm{CHBrCl} 2)(\mu \mathrm{g} / \mathrm{L})$ & 72 & $<0.50$ & $<0.50$ & 1.04 & 1 & 1.39 & 0 & 0 & & 600 & - \\
\hline Dibromochloromethane $(\mu \mathrm{g} / \mathrm{L})$ & 72 & $<0.50$ & $<0.50$ & 1.58 & 1 & 1.39 & 0 & 0 & & 60 & - \\
\hline Tribromomethane $(\mu \mathrm{g} / \mathrm{L})$ & 72 & $<0.50$ & $<0.50$ & 0.81 & 1 & 1.39 & 0 & 0 & & 80 & - \\
\hline \multicolumn{12}{|c|}{ Dissolved gases } \\
\hline Methane (mg/L) & 72 & $<0.0002$ & 0.006 & 77 & 64 & 88.9 & 9 & 12.5 & . & $3>7$ & - \\
\hline Ethane $(\mathrm{mg} / \mathrm{L})$ & 72 & $<0.0002$ & 0.0002 & 0.13 & 15 & 20.8 & - & - & - & - & - \\
\hline Propane (mg/L) & 72 & $<0.0002$ & 0.0002 & 0.001 & 2 & 2.78 & - & - & - & - & - \\
\hline$\delta^{13} \mathrm{C}\left(\right.$ per mil in $\left.\mathrm{CH}_{4}\right)$ & 6 & -65.05 & -44.37 & -38.23 & 6 & 100 & - & - & - & - & - \\
\hline$\delta \mathrm{D}\left(\right.$ per mil in $\left.\mathrm{CH}_{4}\right)$ & 6 & -257.2 & -203.65 & -191.2 & 6 & 100 & - & - & - & - & - \\
\hline
\end{tabular}

'Proposed EPA MCL for states without an enhanced indoor air program.

${ }^{2}$ Volatile organic compounds (VOCs) with detections are shown. A full listing of all VOCs sampled but not detected are shown in Appendix 4.

${ }^{3}$ Pennsylvania action level for methane.

\section{EXPLANATION}


resources engineers commonly identify the potential for encrustation or corrosion on the basis of the Langelier Index (LI), which provides an indication for the potential for lead and copper to enter water supplies from pipes and plumbing (Snoeyink and Jenkins, 1981). The LI, which is the difference between the measured $\mathrm{pH}$ and the $\mathrm{pH}$ at equilibrium with calcium carbonate $\left(\mathrm{CaCO}_{3}\right)$, is equal in value to the calcite saturation index $\left(\mathrm{SI}_{\mathrm{CAL}}\right)$, evaluated in more detail later. If the LI or $\mathrm{SI}_{\mathrm{CAL}}$ is positive, the $\mathrm{pH}$ is greater than that at equilibrium with
$\mathrm{CaCO}_{3}$ and the water will tend to deposit $\mathrm{CaCO}_{3}$ coatings or scale that can insulate pipes, boilers, and other components of a system from contact with water; however, if negative, then the water is undersaturated with $\mathrm{CaCO}_{3}$ and will tend to be corrosive in the distribution system. An optimum condition is desired whereby the LI or $\mathrm{SI}_{\mathrm{CAL}}$ is close to zero, whereby the water will neither be strongly corrosive nor scale forming. For the 72 groundwater samples, $\mathrm{SI}_{\mathrm{CAL}}$ ranged from -2.6 to 0.8 . Of the 72 samples, 11 (2.8 percent) had $\mathrm{SI}_{\mathrm{CAL}}$ values that were
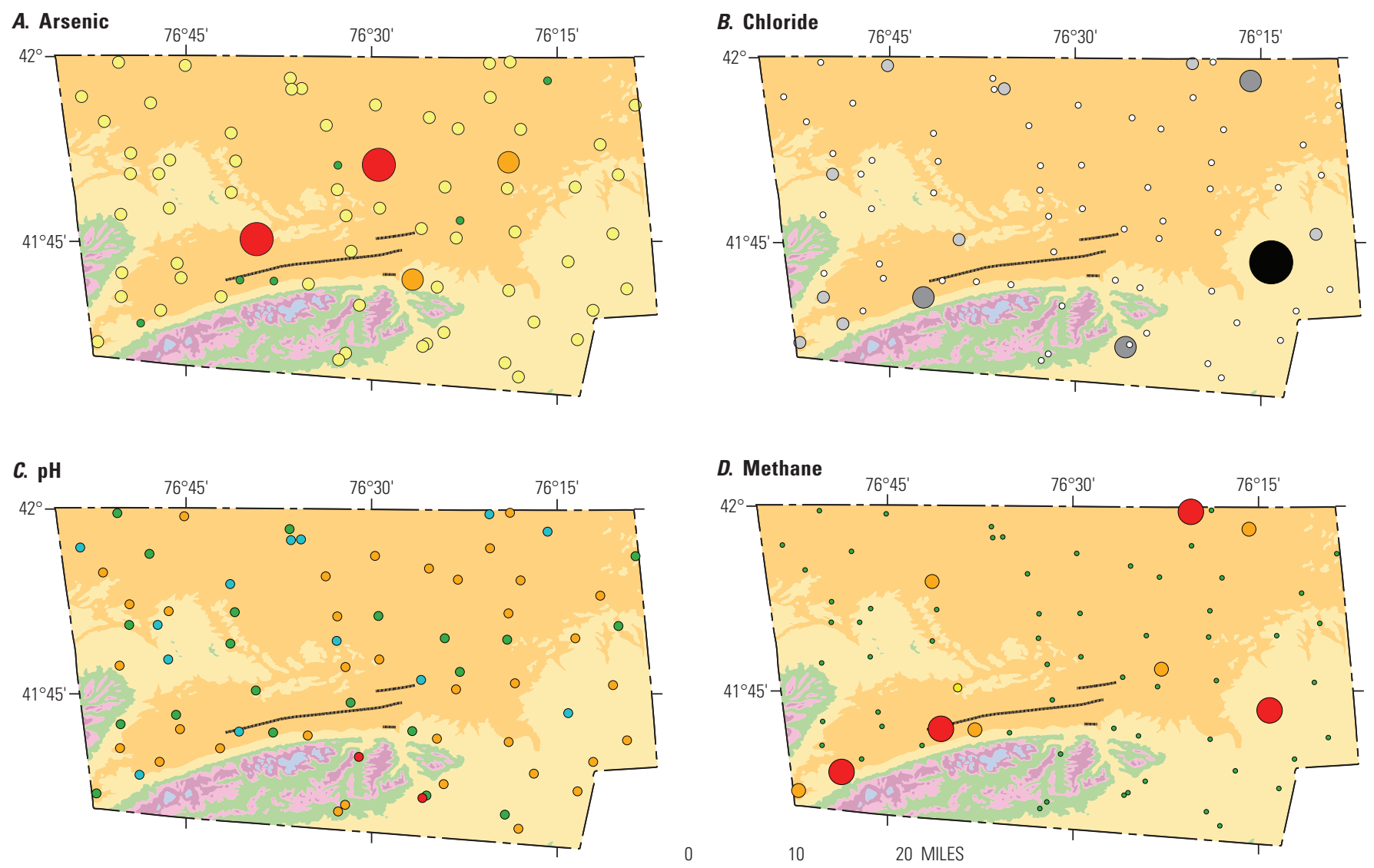

Base from U.S. Bureau of the Census digital data, 2000

1:100,000-scale Albers equal-area projection

Standard parallels $40^{\circ} \mathrm{N}$ and $42^{\circ} \mathrm{N}$,

central meridian $-78^{\circ} \mathrm{W}$, latitude of origin $39^{\circ}$

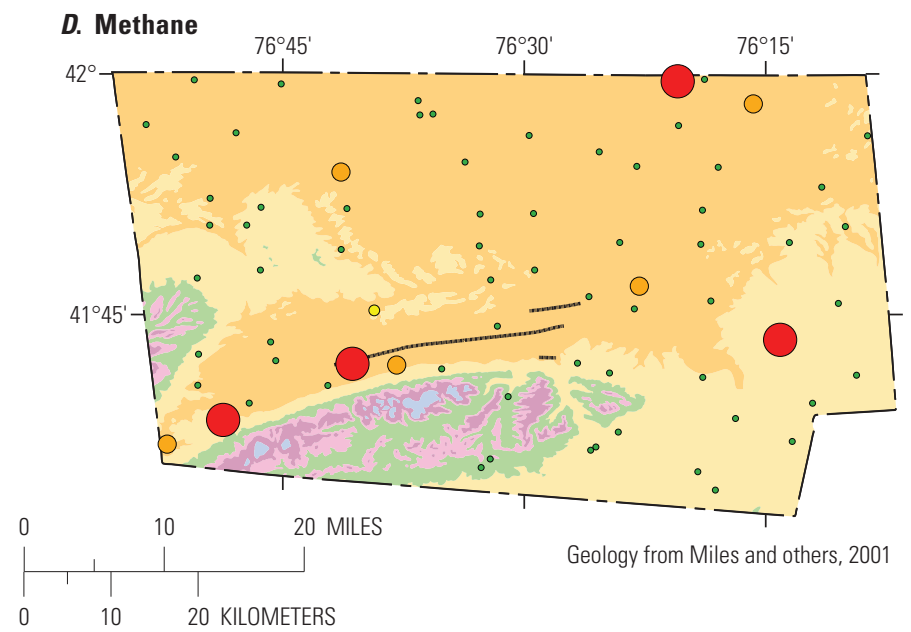

EXPLANATION

\begin{tabular}{l} 
Allegheny and Pottsville Formation \\
Pottsville Formation \\
Burgoon Sandstone \\
\hline Fault \\
Chloride concentration, in \\
milligrams per liter \\
$\quad \leq 25$ \\
$0 \quad>25$ to 100 \\
$>100$ to 500 \\
$>500$
\end{tabular}

Huntley Mountain Formation

Catskill Formation

Lock Haven Formation

$\mathrm{pH}$, in standard units

- $\leq 6.50$

○> 6.50 to 7.50

- $>7.50$ to 8.00

$\bigcirc>8.00$
Methane concentration, in milligrams per liter

- $\leq 2.0$

- $>2.0$ to 10

$>10$ to 26

Figure 3. Spatial distribution of $A$, arsenic concentrations, $B$, chloride concentrations, $C, \mathrm{pH}$ values, and $D$, methane concentrations in 72 wells sampled and bedrock geology in Bradford County, Pennsylvania. ( $\leq$, less than or equal to; $>$, greater than) 
less than -0.5 , indicating potentially strongly corrosive characteristics; the remaining samples would be considered neither strongly corrosive nor scale forming. Two samples characterized as strongly corrosive, BR 893 and BR 952, had SI ${ }_{\mathrm{CAL}}$ less than -1.0 and hardness less than $30 \mathrm{mg} / \mathrm{L}$ as $\mathrm{CaCO}_{3}$, but $\mathrm{pH}$ values were 8.1 and 7.0 , respectively.

\section{Nutrients}

Nutrients include nitrogen and phosphorous species. Nitrogen is found in water principally as nitrate $\left(\mathrm{NO}_{3}\right)$, nitrite $\left(\mathrm{NO}_{2}\right)$, and ammonia $\left(\mathrm{NH}_{4}\right)$, whereas phosphorus is present mainly as orthophosphate $\left(\mathrm{PO}_{4}\right)$. Nutrients are essential for plant growth; however, the presence of elevated nutrient concentrations generally is an indicator of anthropogenic sources, which might include fertilizers, storm runoff, animal wastes, and effluent from septic systems. Summary statistics for nutrients are given in table 1. Nitrate is the most prevalent nitrogen species in oxic groundwater, but all nitrate concentrations were still less than the EPA MCL of $10 \mathrm{mg} / \mathrm{L}$ for nitrate as $\mathrm{N}$ (table 1). Excessive nitrate in drinking water causes a health risk, especially in infants, because it disrupts oxygen flow in blood. Infants below the age of six months who drink water containing nitrate in excess of the MCL could become seriously ill, develop symptoms including shortness of breath, and blue-baby syndrome. Because concentrations of nitrite are so low, nitrate plus nitrite concentrations essentially represent nitrate concentrations. The concentration of nitrate in samples ranged from less than 0.04 to $7.01 \mathrm{mg} / \mathrm{L}$ as $\mathrm{N}$; the median concentration was less than $0.04 \mathrm{mg} / \mathrm{L}$ as N. The concentration of orthophosphate ranged from less than 0.004 to $0.16 \mathrm{mg} / \mathrm{L}$ with a median of $0.01 \mathrm{mg} / \mathrm{L}$.

\section{Bacteria}

All samples were analyzed for total coliform and Escherichia coli (E. coli) bacteria to determine the associated human-health risk of potentially pathogenic (disease-causing) waterborne microorganisms. Coliform bacteria are ubiquitous in the environment and are not always pathogenic. Some strains of E. coli, such as O157:H7, can cause severe illness, and the presence of $E$. coli may indicate general fecal contamination because of the strong association of $E$. coli with the intestines of warm-blooded animals. Any detection of $E$. coli in public drinking-water supplies is considered cause for concern and a violation of health standards (U.S. Environmental Protection Agency, 2001), and these EPA criteria can be used to provide a reference point for domestic wells.

Total coliform bacteria were detected in 35 of the 71 groundwater samples (48.6 percent) (table 1). Total coliform concentrations ranged from less than 1 colony (reported as most probable number [MPN] per 100 milliliters [mL]) to greater than $2,420 \mathrm{MPN} / 100 \mathrm{~mL}$. Of the 35 samples that had total coliform bacteria, 6 samples had detectable $E$. coli, with concentrations ranging from 1 to greater than 2,420
MPN/100mL. The most probable number could not be determined for BR 890, but a present/absent test with the lab determined this sample was absent of total coliform and E. coli bacteria, and for the summary table (table 1) this sample was represented as less than 1 MPN per 100 milliliters. No value was available for BR 928.

\section{Metals and Trace Elements}

Metals and other trace elements typically are present in concentrations less than $1 \mathrm{mg} / \mathrm{L}$ in natural waters (Hem, 1985). Most metals and trace elements in groundwater are leached from soil or dissolved from underlying bedrock in minute quantities by groundwater. Some are present in precipitation. Summary statistics for metals and trace elements are listed in table 1.

The EPA has established MCLs and SMCLs for various metals and trace elements in drinking water (table 1). Two samples (2.8 percent) exceeded the EPA MCL for arsenic and barium, 8 samples (11.1 percent) exceeded the SMCL for dissolved iron, 22 samples (30.6 percent) exceeded the SMCL for dissolved manganese, and 1 sample exceeded the SMCL for aluminum with a value of $770.0 \mu \mathrm{g} / \mathrm{L}$. None of the samples exceeded the EPA MCL or SMCL for antimony, beryllium, cadmium, chromium, copper, lead, selenium, silver, or zinc (table 1).

Arsenic concentrations ranged from less than 0.05 to $23.2 \mu \mathrm{g} / \mathrm{L}$ with a median concentration of $0.63 \mu \mathrm{g} / \mathrm{L}$ (table 1 ). Arsenic concentrations were locally elevated and were not consistently related to $\mathrm{pH}$ (fig. 4A). Two samples, BR 907 and BR 929, had arsenic concentrations exceeding the EPA MCL of $10 \mu \mathrm{g} / \mathrm{L}$ and had a $\mathrm{pH}$ of 7.7 and 8.0, respectively. Possible health effects associated with ingestion of drinking water with arsenic in excess of the MCL for many years include skin damage, circulatory system problems, and increased cancer risk. Arsenate $\left(\mathrm{As}^{\mathrm{V}} \mathrm{O}_{4}{ }^{3-}\right)$ and arsenite $\left(\mathrm{As}^{\mathrm{III}} \mathrm{O}_{3}{ }^{3-}\right)$, which are the predominant forms of arsenic in groundwater, tend to adsorb to a variety of aquifer materials, including iron oxides, aluminum oxides, and clay minerals, at mildly acidic to neutral $\mathrm{pH}$, but not at alkaline $\mathrm{pH}$ conditions (Dzombak and Morel, 1990; Smedley and Kinniburgh, 2002). Furthermore, under strongly acidic or reducing conditions, oxide minerals may become unstable and dissolve, releasing arsenic to solution. More detailed explanation of the effects of $\mathrm{pH}$ on sorption processes and the possible relations between redox state and arsenic concentration are presented in a later section of this report.

Although the highest concentrations of bromide $(8.60 \mathrm{mg} / \mathrm{L})$ and sodium $(760 \mathrm{mg} / \mathrm{L})$ were found in samples having $\mathrm{pH}$ greater than 7.5 (fig. 4), most high $\mathrm{pH}$ samples did not have elevated concentrations of these constituents. In contrast, the highest concentrations of lead $(2.61 \mu \mathrm{g} / \mathrm{L})$, copper $(82.3 \mu \mathrm{g} / \mathrm{L})$, zinc $(981 \mu \mathrm{g} / \mathrm{L})$, and other trace metals were found in samples that had $\mathrm{pH}$ less than 7.5 (fig. $4 G-H$ ). Generally, decreased concentrations of trace metals with increased $\mathrm{pH}$ may be explained by their strong adsorption at alkaline $\mathrm{pH}$ 
A

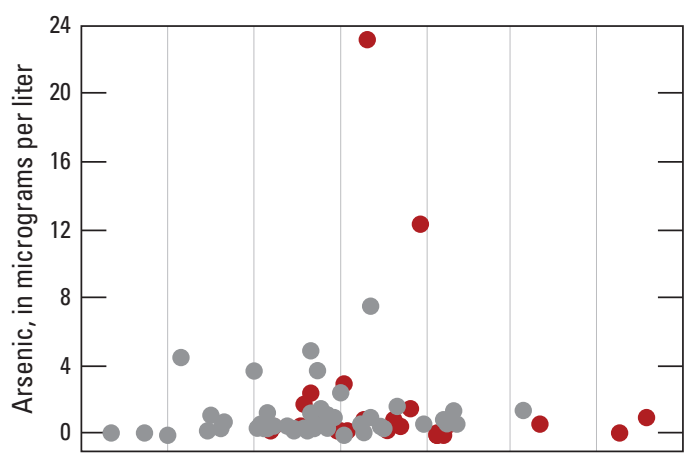

C

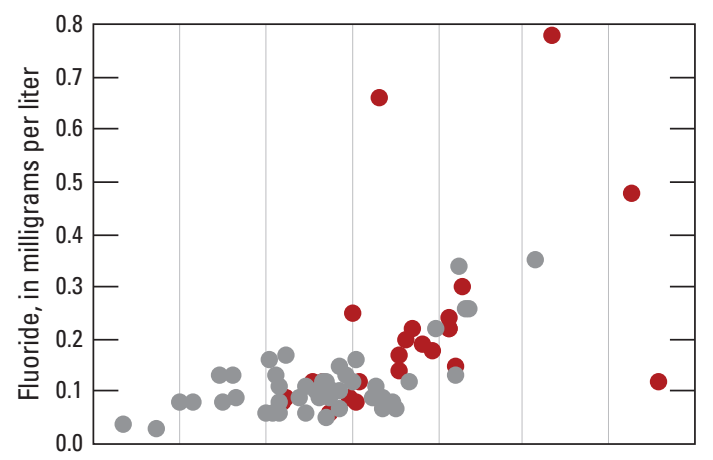

$E$

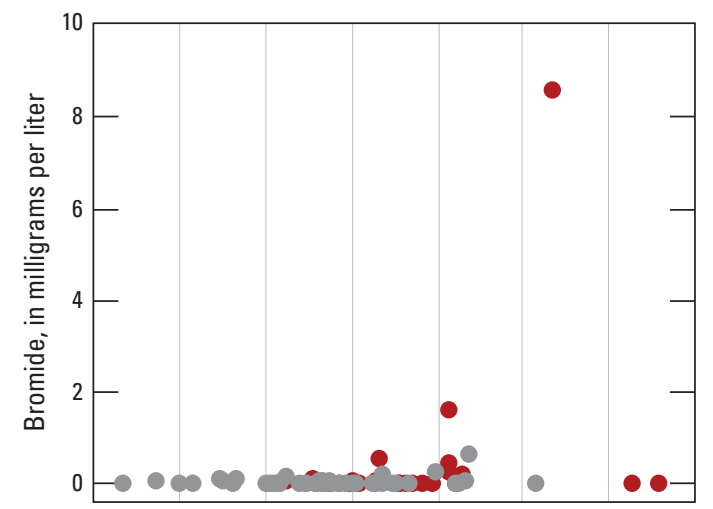

$\boldsymbol{G}$

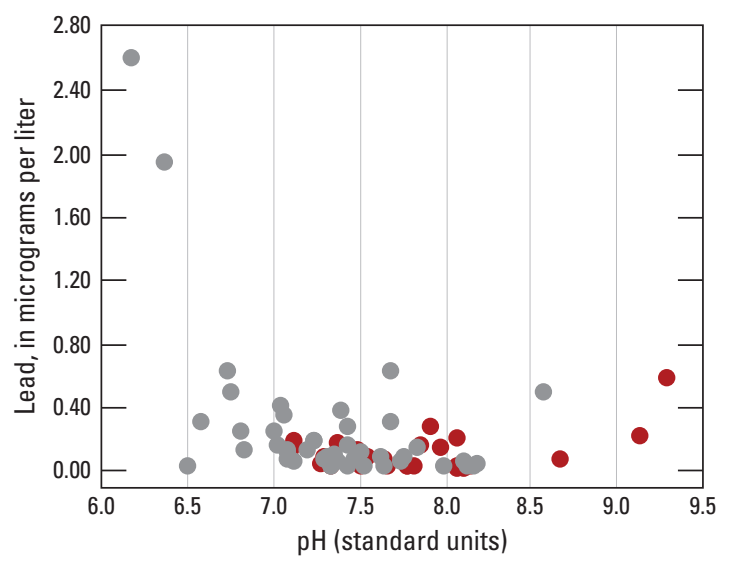

B

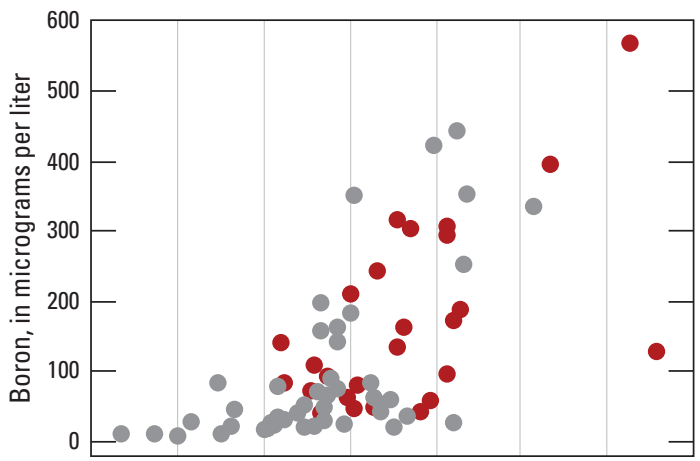

D

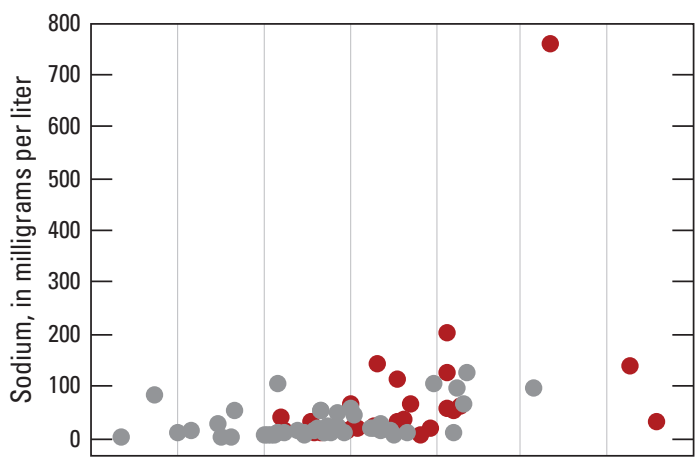

$\boldsymbol{F}$

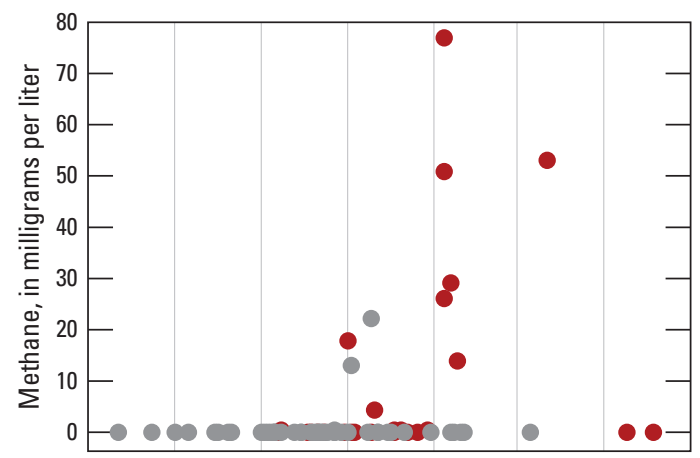

H

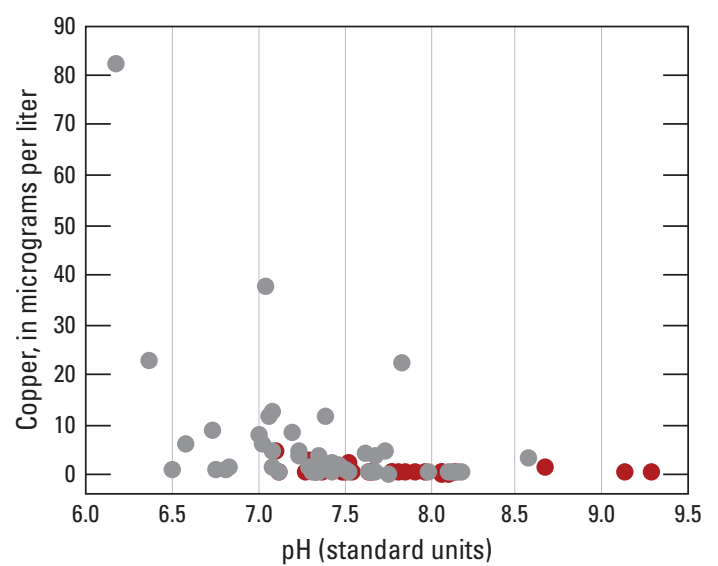

\section{EXPLANATION}

Groundwater-Dissolved oxygen $\geq 0.5 \mathrm{mg} / \mathrm{L}$

- Groundwater-Dissolved oxygen $<0.5 \mathrm{mg} / \mathrm{L}$

Figure 4. Relation between $\mathrm{pH}$ and selected constituents for 72 groundwater samples in Bradford County, Pennsylvania. $\geq$, greater than or equal to; $<$, less than) 
by iron oxides, whereas the increased concentrations of trace anions, such as arsenic or boron (present as borate), may result from their desorption at alkaline $\mathrm{pH}$, explained in more detail further on. The increased concentrations of sodium could result from cation-exchange reactions, also explained further on.

Elevated concentrations of iron and manganese in water may impart a bitter taste and stain laundry and plumbing fixtures with a yellowish or brownish-orange color. Concentrations of dissolved iron ranged from less than 4.0 to $1,830 \mu \mathrm{g} / \mathrm{L}$ with a median of $11.34 \mu \mathrm{g} / \mathrm{L}$. Eight of these samples (11.1 percent) exceeded the EPA SMCL of $300 \mu \mathrm{g} / \mathrm{L}$ for iron. Concentrations of dissolved manganese in water ranged from less than 0.40 to $1,120 \mu \mathrm{g} / \mathrm{L}$; the median concentration was $10.5 \mu \mathrm{g} / \mathrm{L}$. Water samples from 22 of the 72 sampled wells (30.6 percent) exceeded the EPA SMCL of $50 \mu \mathrm{g} / \mathrm{L}$.

Although none of the groundwater samples had concentrations of lead or copper in excess of the respective MCL and SMCL values of 15 and $1,000 \mu \mathrm{g} / \mathrm{L}$, corrosive water, as previously described by the LI or $\mathrm{SI}_{\mathrm{CAL}}$ less than 0.5 , could acquire metals from lead or copper pipes in the household water system. Long-term exposure to excess copper can cause liver and kidney damage, whereas lead can cause neurological problems, especially in young children. Depending on water treatment, the corrosive characteristics may or may not be mitigated. Sampling at the tap would be advisable to evaluate the actual concentrations of constituents in household drinking water and to evaluate the potential effects of water treatment. Lead and copper levels could be much higher if samples were collected before the plumbing system is flushed, as in this study.

\section{Radionuclides}

Radionuclides naturally present in rocks and soils may be dissolved or leached into groundwater. Analyses for radioactivity and radionuclides include gross alpha radioactivity, gross beta radioactivity, and dissolved radon-222 (radon gas). Uranium, a radioactive element, also was analyzed in the dissolved form. Naturally occurring radioactivity is described in detail in previous reports on groundwater quality in Wayne, Pike, and Lycoming Counties in Pennsylvania (Senior and others, 2017; Senior and Cravotta, 2017; and Gross and Cravotta, 2017, respectively). Summary statistics for radioactive constituents in groundwater samples from Bradford County as well as the established or proposed EPA MCLs for some of these constituents are given in table 1 .

The activities of radon-222 in water from the 72 sampled wells ranged from 34.4 to $8,540 \mathrm{pCi} / \mathrm{L}$ with a median activity of $614 \mathrm{pCi} / \mathrm{L}$ (table 1). The EPA does not regulate radon-222 in drinking water. However, under the framework specified by the 1999 Notice for the Proposed Radon in Drinking Water Rule (Federal Register, 1999), the EPA proposed an alternative maximum contaminant level (AMCL) of $4,000 \mathrm{pCi} / \mathrm{L}$ for radon-222 for community water systems that use groundwater for all or some of the supply in states with an enhanced indoor air radon program. For states without an enhanced indoor air program, the EPA proposed an MCL of $300 \mathrm{pCi} / \mathrm{L}$ for radon222. Water samples from 50 of the 72 wells sampled (70.4 percent) exceeded the proposed EPA MCL of $300 \mathrm{pCi} / \mathrm{L}$, and only one sample exceeded the proposed EPA AMCL of $4,000 \mathrm{pCi} / \mathrm{L}$ for radon-222.

The gross alpha-particle radioactivity (72-hour count) in water from the 72 sampled wells ranged from -0.33 to $11.2 \mathrm{pCi} / \mathrm{L}$; the median activity level was $1.7 \mathrm{pCi} / \mathrm{L}$ (table 1 ). Alpha particles are counted at 72 hours and 30 days because some alpha-particle emitters, such as radium-224 (half-life of 3.6 days), would not be present in the 30-day count. Gross alpha-particle activity in the 30-day count was similar to or slightly less than the activity in the 72-hour count. No water sample exceeded the EPA MCL of $15 \mathrm{pCi} / \mathrm{L}$ for gross alphaparticle activity (table 1).

The gross-beta particle radioactivity (72-hour count) ranged from 0.03 to $5.89 \mathrm{pCi} / \mathrm{L}$. Gross beta-particle activity in the 30-day count was similar to or slightly less than the activity in the 72-hour count in about half of the samples (table 1). The concentration of uranium ranged from less than 0.01 to $10.4 \mu \mathrm{g} / \mathrm{L}$. The median concentration was $0.26 \mu \mathrm{g} / \mathrm{L}$. No water samples exceeded the EPA MCL of $30 \mu \mathrm{g} / \mathrm{L}$ for uranium.

A subset of eight samples, which had the highest grossalpha and gross-beta activities of the 72 wells sampled, was analyzed for radium-226 and radium-228. Concentrations (activities) of radium-226 ranged from 0.16 to $2.08 \mathrm{pCi} / \mathrm{L}$ in water samples, and activities of radium-228 ranged from -0.02 to 1.54 from the eight wells. None of the samples exceeded the EPA MCL of $5 \mathrm{pCi} / \mathrm{L}$ for combined radium-226 and radium-228. The highest activities of radium-226 and radium-228 were measured for the same sample, BR 921. This sample also had the highest 30-day gross alpha and 30-day gross beta activities of the 72 samples reported herein.

\section{Dissolved Methane and Other Naturally Occurring Hydrocarbon Gases}

Water sampled from the 72 wells had concentrations of dissolved methane ranging from less than 0.0002 to $77 \mathrm{mg} / \mathrm{L}$, plus lower to nondetectable concentrations of other, more complex hydrocarbon gases, including ethane, ethylene, propane, propylene, iso-butane, and N-butane (in order of increasing number of hydrocarbon chains) (table 1). None of the samples had detectable concentrations of the dissolved hydrocarbons iso-pentane, N-pentane, and hexane.

Detectable levels of methane were found in water from 64 of the 72 wells sampled (88.9 percent) for which concentrations were greater than the reporting level of $0.0002 \mathrm{mg} / \mathrm{L}$ (table 1). Nine of the samples had dissolved methane concentrations that exceeded the Pennsylvania action level of greater than $7 \mathrm{mg} / \mathrm{L}$ (Commonwealth of Pennsylvania, 2014). Most samples with detectable concentrations of methane had alkaline $\mathrm{pH}$; the 10 water samples with highest methane 
concentrations had $\mathrm{pH}$ greater than 7.5 (fig. $4 F$ ). Eight of these 10 samples also had low concentrations of dissolved oxygen (less than $0.5 \mathrm{mg} / \mathrm{L}$ ) (fig. $4 F$ ).

\section{Man-Made Organic Compounds}

All samples were analyzed for selected man-made organic compounds, including VOCs, glycols and alcohols, and oil and grease (table 1). Out of these man-made organic compounds, only three were measured in detectable concentrations in 1 of the 72 wells sampled.

VOCs include a wide range of natural and synthetic carbon-based compounds that have high vapor pressure and relatively low solubility in water. VOCs are used in industrial, commercial, and domestic applications and can enter the groundwater as liquid through spills and leaks or by atmospheric deposition. VOCs typically found in groundwater include industrial solvents, fuel hydrocarbons and oxidizers, fumigants, organic synthesis compounds, refrigerants, and disinfection byproducts (trihalomethanes) (Carter and others, 2010). VOCs are the most commonly found contaminant class associated with industrial and commercial sites, dumps, landfills, hazardous waste facilities, and military bases (Zogorski and others, 2006) and are widespread where other human activities occur in developed countries. Historically, many waste chemicals were disposed of indiscriminately, and the widespread use of VOCs has resulted in a considerable mass of material released to the environment. Localized releases of VOCs occur from many sources, including leakage from storage tanks, direct application of pesticides containing VOCs (Barbash and Resek, 1996), septic systems, and leaking sewer systems. VOCs also are released to the atmosphere through engine exhausts, aerosol sprays, leakage of refrigerants, and application of fumigants and pesticides. Trihalomethanes are frequently associated with chlorinated water and chlorinated cleaning products.

The reporting level for VOCs was $0.5 \mathrm{mg} / \mathrm{L}$, which is lower than drinking-water MCLs established for 14 of the 68 analyzed VOCs (U.S. Environmental Protection Agency, 2012a). However, drinking-water MCLs for two compounds1,2-dibromo-3-chloropropane (DBCP) and 1,2-dibromoethane (EDB) - are less than the method reporting level as performed by the laboratory, so for these compounds, a more sensitive method is needed to determine if they are present in concentrations greater than the MCLs (although below the reporting level of $0.5 \mathrm{mg} / \mathrm{L}$ ). Therefore, with the exception of these two compounds, if VOCs are present in groundwater at concentrations less than the reporting level of $0.5 \mathrm{mg} / \mathrm{L}$, those concentrations would not exceed an established drinking-water standard. MCLs have not been established for VOCs where data are insufficient to evaluate human-health effects.

Of the 68 VOCs analyzed in the 72 groundwater samples, only 3 trihalomethanes were detected above the reporting level: bromodichloromethane $(1.04 \mu \mathrm{g} / \mathrm{L})$, dibromochloromethane $(1.58 \mu \mathrm{g} / \mathrm{L})$, and tribromomethane $(0.81 \mu \mathrm{g} / \mathrm{L})$. All three VOCs were detected in samples from the same well, BR 913, but concentrations of the detected VOCs did not exceed any established EPA drinking-water standard. Although the measured VOC concentrations do not pose any established health risk, the detection of these man-made compounds in groundwater indicates a possible low level of groundwater contamination by human activities.

Glycols and alcohols, and oil and grease, were less than the $5 \mathrm{mg} / \mathrm{L}$ reporting level. Although lower concentrations of these constituents may be present in the sampled groundwater, at the time of this study, a more sensitive approved method with lower reporting levels was not available. As of 2018, there are no established EPA drinking-water standards for oil and grease.

\section{Chemical Analysis and Relations Among Constituents in Groundwater}

Dissolved constituents in groundwater may be derived from atmospheric, geologic, biologic, and anthropogenic sources as the aqueous solution interacts with various materials along transport pathways. Solute concentrations can range widely depending on the presence of constituent elements in the source(s), the extent of contact between water and the source, the aqueous solubility and interactions among the dissolved elements, plus geochemical conditions such as $\mathrm{pH}$ and oxidation-reduction (redox) state that affect element form, mobility, and transport in the aqueous environment.

\section{Conceptual Hydrogeochemical Model}

A conceptual hydrogeochemical model that indicates spatial relations among the salinity, water types, redox, and methane sources associated with shallow, intermediate, and deep groundwater flow systems for Bradford and surrounding counties is described in Gross and Cravotta (2017). Most wells constructed for domestic use are completed within the local freshwater-flow system. The wells sampled in Bradford County for this study were completed to depths ranging from 42 to 600 feet, with half of the wells drilled to depths of 175 to 400 feet. Several wells with depths ranging from 165 to 300 feet exhibited characteristics of brine-influenced waters with characteristics of elevated SC and TDS dominated by sodium and chloride, plus elevated concentrations of bromide, lithium, and methane, as explained in more detail below.

\section{Relations Among pH, Specific Conductance, and Constituent Concentrations}

Evaluation of the correlations among chemical constituents and environmental variables provides insight on hydrochemical processes affecting groundwater chemistry in the study area. The $\mathrm{pH}, \mathrm{SC}$, and associated chemical compositions 
of the 72 groundwater samples collected in Bradford County during 2016 ranged widely, as indicated by $\mathrm{pH}$ values from 6.18 to 9.31 and SC from 72.4 to $3,410 \mu \mathrm{S} / \mathrm{cm}$ (fig. 5). Most of the samples had $\mathrm{pH}$ values from 7 to 8.2 and $\mathrm{SC}$ from 300 to $550 \mu \mathrm{S} / \mathrm{cm}$. Only 3 of the 72 well-water samples in this study had SC values greater than $1,000 \mu \mathrm{S} / \mathrm{cm}$. In addition to figure 5 , which shows the relations among $\mathrm{pH}, \mathrm{SC}$, and multiple inorganic constituents, boxplots are included in the appendix that show the overall relations among individual constituents with pH class interval (fig. 4.1) and SC class interval (fig. 4.2). Additional boxplots show the same constituents as a function of redox class interval (fig. 4.3), bedrock formation (fig. 4.4), and topographic position index (fig. 4.5).

The $\mathrm{pH}$ for the 72 groundwater samples was positively correlated with SC and other measures of ionic strength, including TDS (TDScalc and ROE), whereas the $\mathrm{pH}$ and SC were negatively correlated with the concentration of DO. With increased $\mathrm{pH}$, the concentrations of several major and trace constituents generally increased, including alkalinity, sodium $(\mathrm{Na})$, lithium (Li), boron (B), fluoride (F), and arsenic (As) (fig. 5, fig. 4.1). Despite positive correlations between $\mathrm{SC}$ and the concentrations of chloride ( $\mathrm{Cl}$ ) and bromide (Br), (fig. 5), those constituents were not positively correlated with $\mathrm{pH}$ over the entire range of measured values (table 3.2). Concentrations of potassium (K) positively correlated with SC, but were not correlated with $\mathrm{pH}$ (table 3.2).

The relation between the $\mathrm{pH}$ and concentrations of $\mathrm{Ca}$, $\mathrm{Mg}$, and hardness changes from positive to negative at about $\mathrm{pH} 7.5$ (fig. 5). For $\mathrm{pH}$ values less than 7.5, the concentrations of hardness, $\mathrm{Ca}, \mathrm{Mg}$, and other cations, including $\mathrm{Sr}, \mathrm{Na}$, $\mathrm{K}, \mathrm{Li}$, and alkalinity, generally increased with $\mathrm{pH}$. However, for $\mathrm{pH}$ values greater than 7.5, the concentrations of hardness, $\mathrm{Ca}, \mathrm{Mg}, \mathrm{Sr}$, and $\mathrm{Ba}$ decreased with increased $\mathrm{pH}$, while $\mathrm{SC}$ and concentrations of Na, alkalinity, and TDS continued to increase. Such patterns in $\mathrm{pH}, \mathrm{SC}$, and constituent concentrations are consistent with the dissolution of calcite $\left(\mathrm{CaCO}_{3}+\mathrm{H}^{+}=\mathrm{Ca}^{2+}+\mathrm{HCO}_{3}^{-}\right)$over the range of $\mathrm{pH}$, combined with cation exchange at $\mathrm{pH}$ greater than 7.5. Through cationexchange reactions, the hardness is removed, or softened, as $\mathrm{Ca}, \mathrm{Ba}, \mathrm{Mg}$, and $\mathrm{Sr}$ ions displace $\mathrm{Na}$ ions from exchange sites on clay minerals $\left(0.5 \mathrm{Ca}^{2+}+\mathrm{NaX}=0.5 \mathrm{CaX}_{2}+\mathrm{Na}^{+}\right)$(Appelo and Postma, 2005), as explained in more detail below.

Boxplots show the constituent concentrations for four different $\mathrm{pH}$ class intervals (fig. 4.1). As indicated by the scatter plots that showed constituent concentrations as a function of $\mathrm{pH}$ for all 72 groundwater samples (fig. 5), most constituent concentrations increased with $\mathrm{pH}$ to values of 7.4 (acidic to neutral range). The most alkaline $\mathrm{pH}$ class $(8.0<\mathrm{pH}<9.4)$ had higher median values for SC, TDS, Na, K, alkalinity, $\mathrm{Cl}, \mathrm{Br}, \mathrm{F}$, $\mathrm{B}, \mathrm{Li}$, and methane than the acidic $(6.1<\mathrm{pH}<6.4)$ or near-neutral $(6.5<\mathrm{pH}<7.4) \mathrm{pH}$ classes. In contrast, the most alkaline $\mathrm{pH}$ class had lower or equal medians for hardness, $\mathrm{Ca}, \mathrm{Mg}$, nitrate $\left(\mathrm{NO}_{3}\right)$, dissolved oxygen, $\mathrm{Zn}, \mathrm{U}$, and $\mathrm{Rn}-222$ compared to the acidic to near-neutral $\mathrm{pH}$ classes.

Boxplots also show constituent concentrations for four different SC class intervals (fig. 4.2). As indicated by the scatter plots that showed constituent concentrations as a function of SC for all 72 groundwater samples (fig. 6), most constituent concentrations increased with $\mathrm{SC}$ to values approaching $700 \mu \mathrm{S} / \mathrm{cm}$. The highest SC class $(600<\mathrm{SC}<3,800 \mu \mathrm{S} / \mathrm{cm})$ had higher median values for $\mathrm{pH}, \mathrm{SC}$, TDS, $\mathrm{Na}, \mathrm{K}$, alkalinity, $\mathrm{Cl}, \mathrm{Br}, \mathrm{F}, \mathrm{B}, \mathrm{Li}$, methane, ammonia $\left(\mathrm{NH}_{3}\right)$, and phosphate $\left(\mathrm{PO}_{4}\right)$, and lower median values for uranium and $\mathrm{Rn}-222$ than the lower SC classes. Constituents that did not vary with SC and (or) $\mathrm{pH}$ may be controlled by other factors, such as redox state.

The constituent concentrations for anoxic, mixed, and oxic redox class intervals also are illustrated as boxplots (fig. 4.3). Although most constituent concentrations did not vary with redox, the medians for several constituents were significantly different between the anoxic and oxic redox classes. The anoxic samples had higher median values for $\mathrm{pH}, \mathrm{Na}, \mathrm{Ba}$, $\mathrm{Sr}, \mathrm{Br}, \mathrm{F}, \mathrm{B}, \mathrm{NH}_{3}, \mathrm{Mo}, \mathrm{Fe}, \mathrm{Mn}$, and methane, and lower median values for $\mathrm{DO}, \mathrm{NO}_{3}, \mathrm{U}$, and $\mathrm{Rn}-222$ than the oxic samples.

\section{Ionic Contributions to Conductivity and Total Dissolved Solids}

Despite general correlations between SC and major ion concentrations, the predominance of various ionic contributions to the $\mathrm{SC}$ varied widely for samples with comparable values of SC (fig. 6). Calcium and bicarbonate were the predominant ions in most samples, considering the ionic contributions to conductivity or considering the ion concentrations in units of mass, moles, or equivalents. Although sodium and chloride were subordinate in most samples, these constituents were the predominant ions in a few samples, particularly those with SC greater than $1,000 \mu \mathrm{S} / \mathrm{cm}$, but also in several samples with relatively low values of SC. Sulfate was subordinate to bicarbonate and chloride as a source of conductivity in the samples; its importance generally decreased with increased SC. Contributions by magnesium were comparable to sulfate; however, contributions by nitrate, potassium, hydrogen, hydroxyl, or carbonate ions were relatively minor.

Variations in the major ion species contributions to the laboratory measured SC values for six selected groundwater samples are illustrated in figure 7 . To provide context, the samples illustrated in figure 7 are identified among all 72 samples in figure 6. Calcium, bicarbonate, magnesium, sodium, sulfate, and chloride ions are the predominant sources of conductivity for the average of the 72 samples and most other samples having low to moderate $\mathrm{SC}$ values (fig. $7 A$, $\mathrm{BR} 952$; fig. $7 B$, BR 963), with minor contributions from nitrate, potassium, and hydrogen ions. Samples with elevated SC values generally have increased contributions from sodium, bicarbonate, and chloride (fig. 7D, BR 941; fig. 7F, BR 913) and, in some cases, sulfate (fig $7 E$, BR 946).

Sample BR 952 (fig. 7A) is representative of minimally evolved groundwaters with a relatively low $\mathrm{SC}$ value of $72 \mu \mathrm{S} / \mathrm{cm}$. The conductivity of this sample can be produced as the result of rainwater evaporation plus dissolution of various 

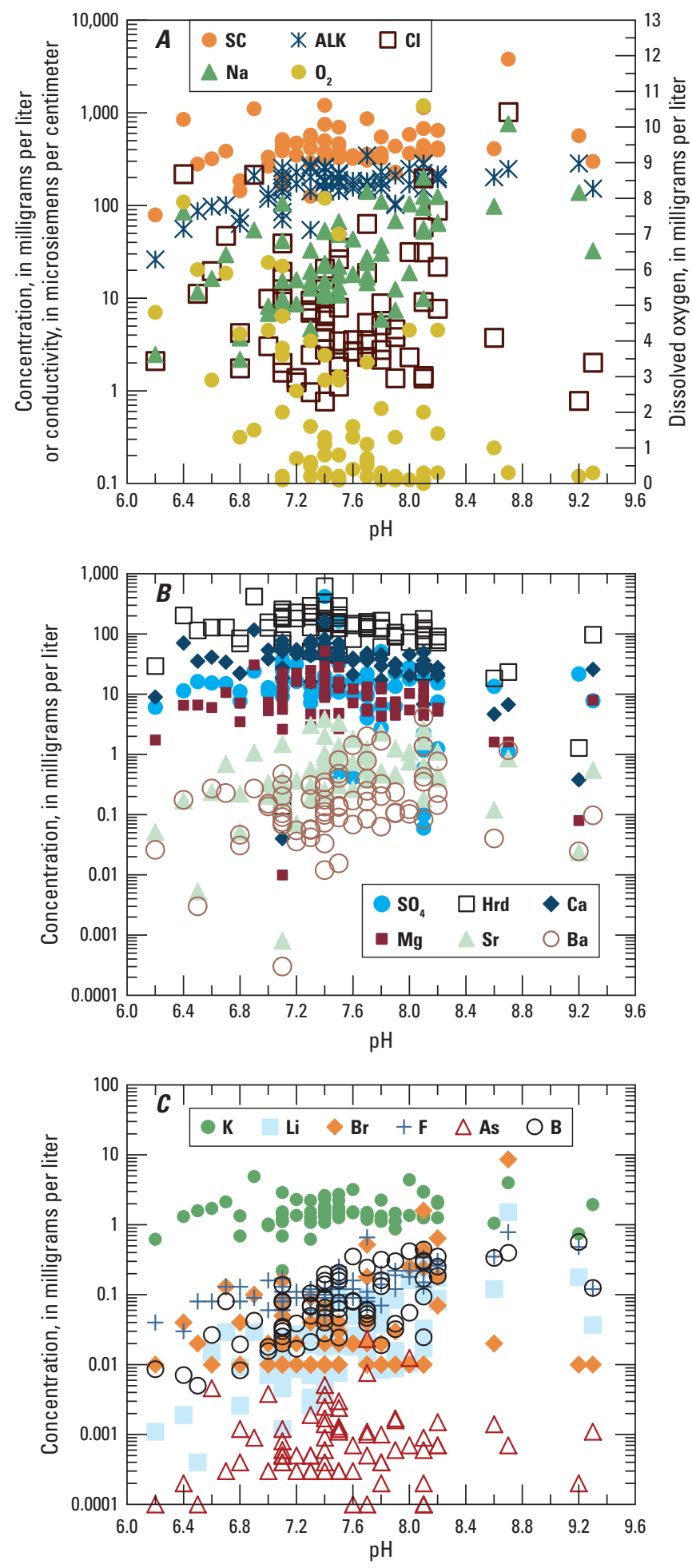

Figure 5. Concentrations of selected constituents in groundwater from Bradford County, Pennsylvania, 2016, compared to: $A-C, \mathrm{pH}$; or $D-F$, specific conductance measured in the laboratory. The detection limits for bromide and arsenic were 0.01 and 0.05 micrograms per liter, respectively; symbols plotted at those values were below detection.
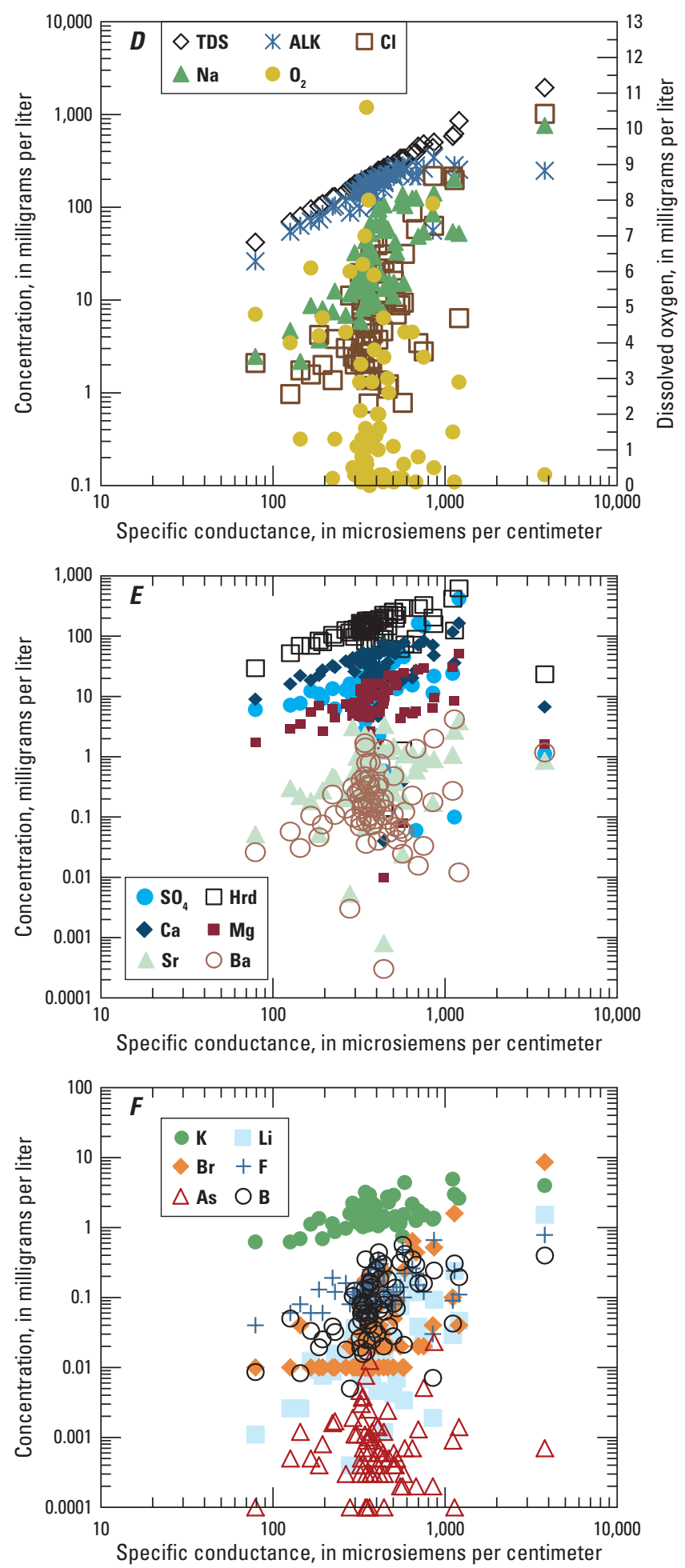

\section{EXPLANATION}

\begin{tabular}{|c|c|c|}
\hline SC-Specific conductance & $\mathbf{S O}_{4}$-Sulfate & - $\mathbf{K}$-Potassium \\
\hline * ALK_Alkalinity & $\square$ Hrd-Hardness & Li-Lithium \\
\hline$\square$ Cl-Chloride & $\diamond \mathrm{Ca}-$ Calcium & $\mathrm{Br}$-Bromide \\
\hline$\Delta \mathrm{Na}-$ Sodium & - $\mathbf{M g}$-Magnesium & $+\mathbf{F}$-Fluoride \\
\hline $\mathbf{O}_{2}-0 x y g e n$ & $\Delta \mathbf{S r}-$ Strontium & $\triangle$ As-Arsenic \\
\hline $\begin{array}{l}\diamond \text { TDS_-Total dissolved solids, } \\
\text { computed as sum } \\
\text { of constituents }\end{array}$ & O Ba-Barium & O B-Boron \\
\hline
\end{tabular}


A

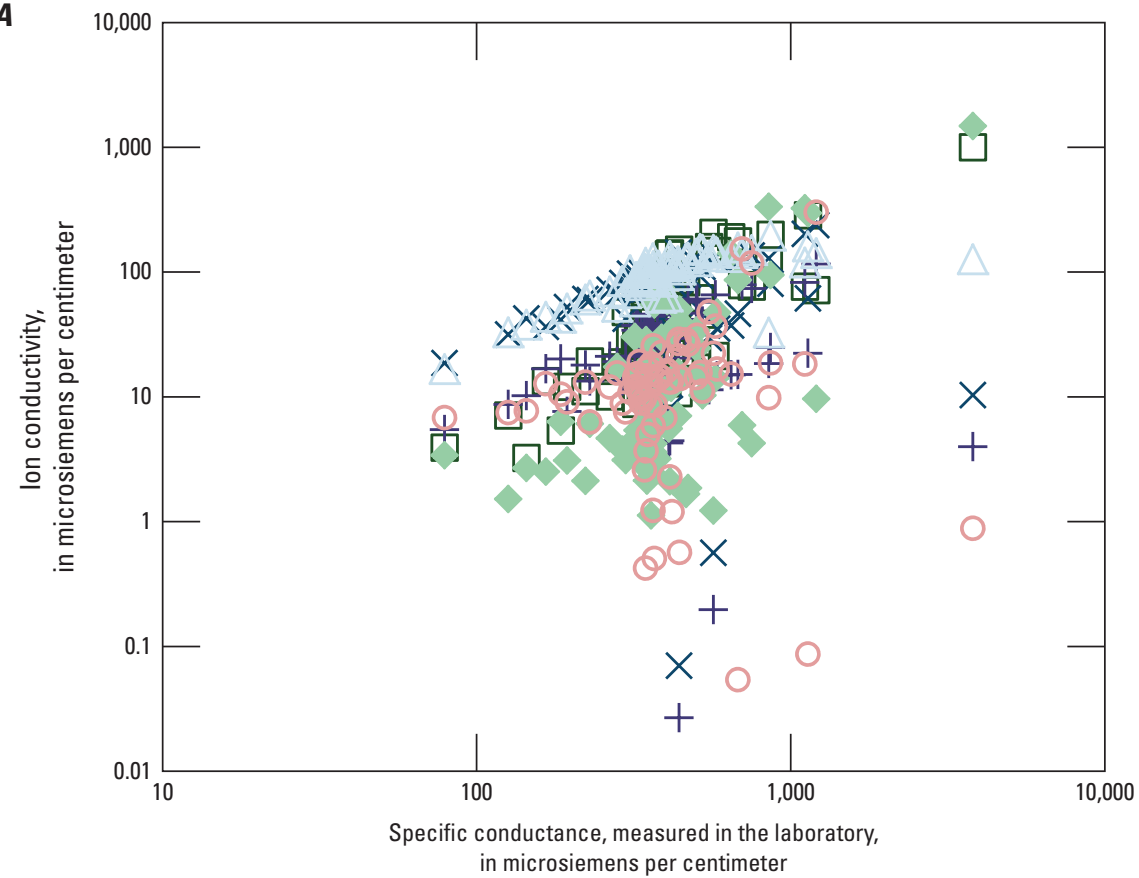

EXPLANATION

$\square$ Sodium

$\times$ Calcium

+ Magnesium

$\checkmark$ Chloride

Bicarbonate

Sulfate

B

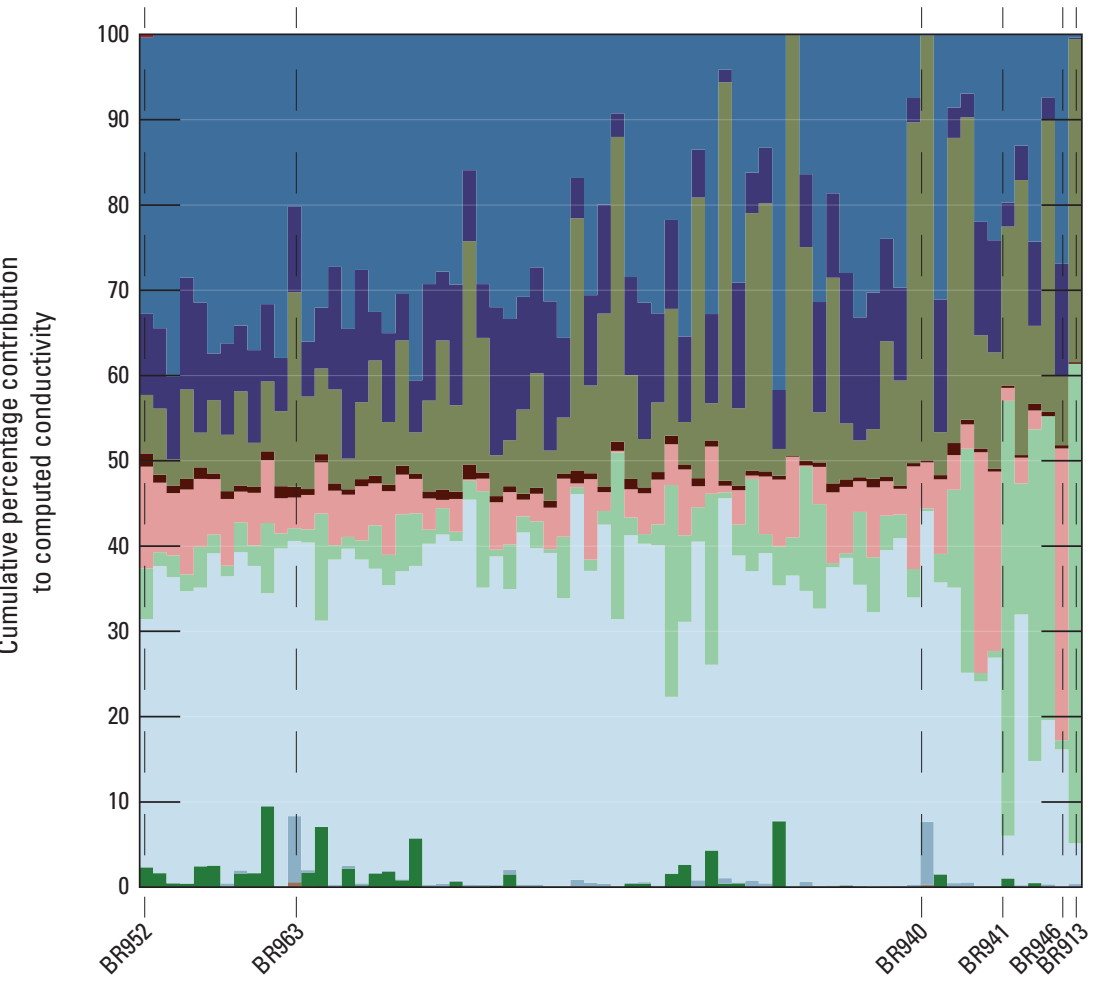

EXPLANATION

Major ions

- $\mathrm{H}^{+}$(Hydrogen)

- $\mathrm{Ca}^{+2}$ (Calcium)

- $\mathrm{Mg}^{+2}$ (Magnesium)

- $\mathrm{Na}^{+}$(Sodium)

- $\mathrm{K}^{+}$(Potassium)

- $\mathrm{SO}_{4}{ }^{-2}$ (Sulfate)

- $\mathrm{Cl}^{-}$(Chloride)

- $\mathrm{HCO}_{3}^{-}$(Bicarbonate)

- $\mathrm{CO}_{3}^{-2}$ (Carbonate)

- $\mathrm{NO}_{3}^{-}$(Nitrate)

- $\mathrm{OH}^{-}$(Hydroxide)

Well Samples

Figure 6. Major ion contributions to specific conductance (SC) for 72 groundwater samples from Bradford County, Pennsylvania, 2016: $A$, comparison of estimated ionic conductivity contributions by sodium, calcium, magnesium, chloride, bicarbonate, and sulfate to measured SC for all 72 samples; and $B$, relative contributions by major ion species to computed SC and selected groundwater samples of representative water types, expressed in percent, in order of increasing SC. Individual ion conductivities estimated from dissolved constituent concentrations as the transport number (the relative contribution of a given ion to the overall conductivity, using the methods of McCleskey and others [2012]) after aqueous speciation calculations with PHREEQC (Parkhurst and Appelo, 2013). 


\section{A. Calcium/bicarbonate type} BR952

$\mathrm{SC}=72 \mu \mathrm{S} / \mathrm{cm}$

$\mathrm{pH}=6.2$

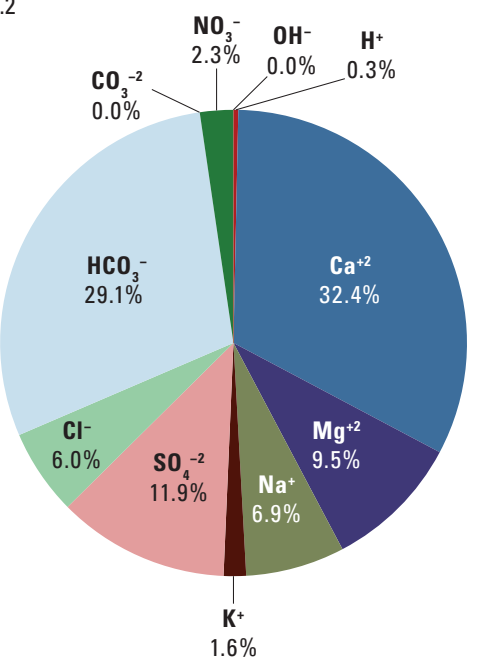

\section{Sodium-calcium/chloride type}

\section{BR941}

$\mathrm{SC}=850 \mu \mathrm{S} / \mathrm{cm}$

$\mathrm{pH}=6.4$

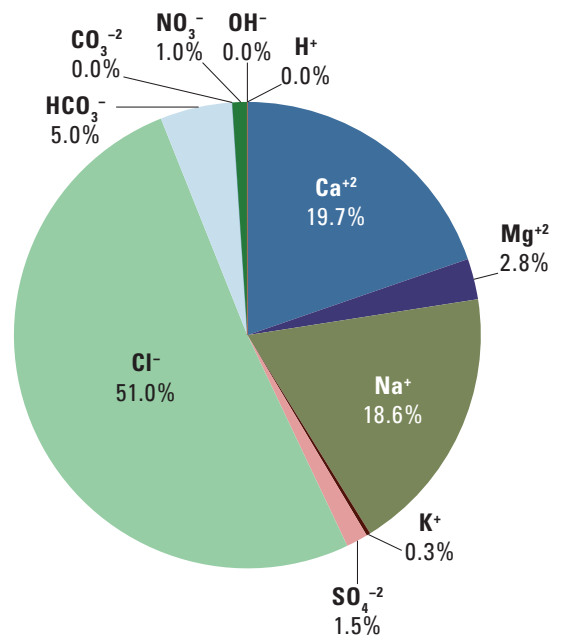

\section{B. Calcium-sodium/bicarbonate type} BR963

$\mathrm{SC}=298 \mu \mathrm{S} / \mathrm{cm}$

$\mathrm{pH}=9.3$

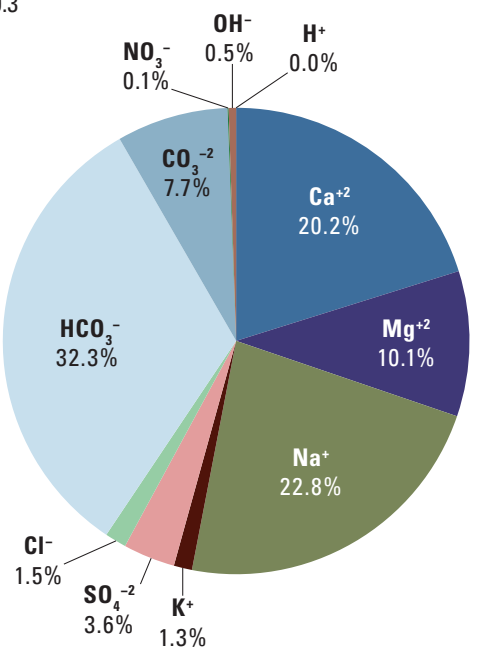

\section{E. Calcium/sulfate-bicarbonate type}

BR946

$\mathrm{SC}=1,210 \mu \mathrm{S} / \mathrm{cm}$

$\mathrm{pH}=7.4$

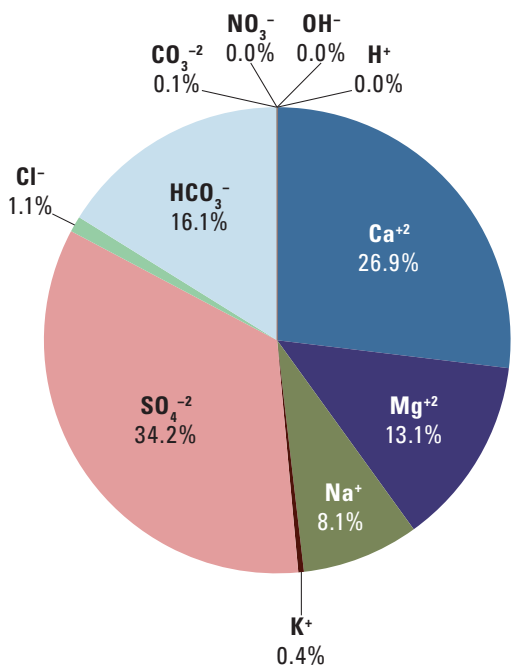

\section{Sodium/bicarbonate type}

BR940

$\mathrm{SC}=567 \mu \mathrm{S} / \mathrm{cm}$

$\mathrm{pH}=9.2$

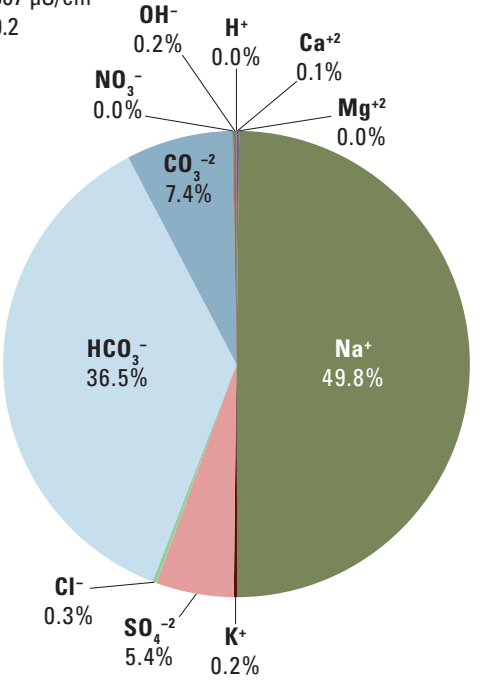

\section{F. Sodium-chloride type}

BR913

$\mathrm{SC}=3,800 \mu \mathrm{S} / \mathrm{cm}$

$\mathrm{pH}=8.7$

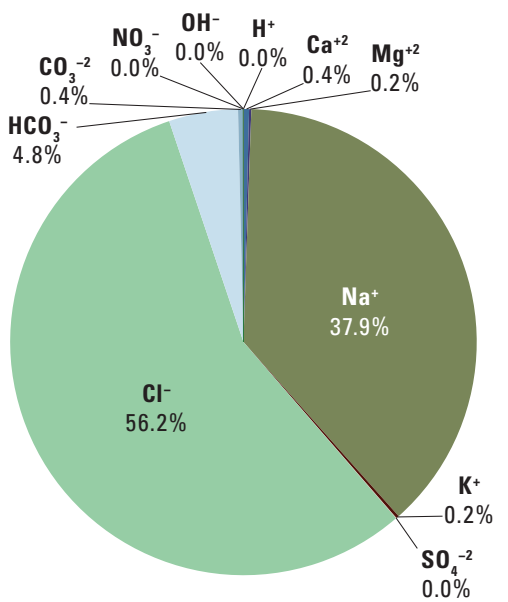

\section{EXPLANATION}

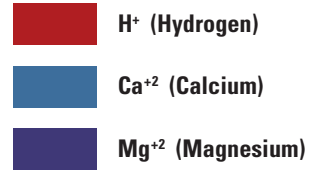

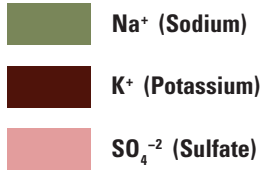

$\mathrm{Cl}^{-}$(Chloride)

$\mathrm{HCO}_{3}^{-}$(Bicarbonate)

$\mathrm{CO}_{3}^{-2}$ (Carbonate)

Figure 7. Water types and ionic contributions to specific conductance (SC) computed for selected groundwater samples from Bradford County, Pennsylvania, 2016. Individual ionic conductivities estimated from dissolved constituent concentrations as the transport number (the relative contribution of a given ion to the overall conductivity, using the methods of McCleskey and others [2012]) after aqueous speciation calculations with PHREEOC (Parkhurst and Appelo, 2013). ( $\mu$ S/cm, microsiemens per centimeter) 
minerals and salts. Sample BR 963 (fig. 7B) had higher $\mathrm{pH}$ of 9.3 and SC of $298 \mu \mathrm{S} / \mathrm{cm}$ compared to BR 952 ; this would be classified as a mixed calcium-sodium/bicarbonate water type. In contrast, sample BR 940 (fig. 7C), which had pH of 9.2 and $\mathrm{SC}$ of $567 \mu \mathrm{S} / \mathrm{cm}$, had predominant ionic conductivity contributions by sodium and bicarbonate, with lesser contributions by chloride, carbonate, and sulfate ions. The mixed calciumsodium/bicarbonate and the sodium/bicarbonate waters can be produced as a result of cation exchange processes, where sodium ions in the mineral are exchanged for calcium ions from the water. Chloride is the dominant anion in sample BR 941 (fig. 7D), whereas sulfate predominates in BR 946 (fig. $7 E$ ). These samples have relatively high $\mathrm{SC}$ values resulting from the addition of these ions to the groundwater. In the extreme, sample BR 913 (fig. 7F), which had pH of 8.7 and the highest measured SC of $3,800 \mu \mathrm{S} / \mathrm{cm}$, had predominant contributions by sodium and chloride, which indicate a salt source. The predominance of sodium and chloride ionic conductivities was exhibited mainly by those samples with elevated $\mathrm{SC}$ but also by a few samples with intermediate $\mathrm{SC}$ values, such as sample BR 926, which had pH of 8.2 and SC of $646 \mu \mathrm{S} / \mathrm{cm}$.

\section{Correlations Among Major and Trace Constituents in Groundwater}

Constituents that were correlated with one another were "loaded" together and separated from other constituents in a principal component analysis (PCA) model (appendix 3). Six principal components ( $\mathrm{pH}$, redox, hardness, chloridebromide, strontium-barium, molybdenum-arsenic) explain nearly 78.3 percent of the variance in the groundwater dataset and consist of 26 routinely detected constituents (table 3.1). The association of $\mathrm{pH}$ with total dissolved solids, alkalinity, sodium, and other parameters is consistent with the progressive reaction of groundwater with minerals along flow paths, where the older water farther from recharge areas becomes more mineralized, alkaline, and softened. The association of redox-sensitive constituents, including dissolved oxygen, nitrate, iron, manganese, and methane, is interpreted to indicate isolation from the atmosphere, the depletion of oxygen, and the development of reducing conditions that may be attributed to the microbial decomposition of organic matter in the aquifer or introduced to the groundwater. The association of hardness, calcium, magnesium, alkalinity, and sulfate results from the dissolution of calcite, dolomite, gypsum, and, possibly, pyrite or other sulfide minerals. Chloride-bromide associations could indicate the influence of Appalachian Basin brine or other sources of salinity, such as sewage, fertilizer, and (or) road-deicing salt, on the groundwater of associated samples. Lastly, associations of the strontium-barium cations and silica could indicate a common origin or geochemical control, such as silicate or carbonate minerals. Associations of molybdenum-arsenic and lack of correlation with other constituents indicates that variations in the trace-element concentrations are independent of variations in the major ions, $\mathrm{SC}, \mathrm{pH}$, or redox.

\section{Relations Between Groundwater Quality, Geology, and Topographic Setting}

Groundwater acquires solutes through natural and anthropogenic loading of constituents in the recharge area from precipitation, weathering reactions of minerals in the soil and aquifer materials, and constituents applied by human activities at or near the land surface. Additional solutes may be acquired as groundwater flows through the aquifer. Groundwater supplying most domestic wells completed in shallow fracturedbedrock aquifers is derived principally from local recharge and will be influenced by land use and geology near the well.

The bedrock underlying Bradford County mainly consists of clastic sedimentary lithologies that include shale, siltstone, and sandstone. Such clastic rocks are mainly composed of silicate and aluminosilicate minerals, including quartz, feldspar, chlorite, muscovite, and illite, plus minor carbonate, sulfate, sulfide, and oxide minerals that occur as clasts, fracture filling, and cements. Although mineralogy is expected to vary locally, the carbonate, sulfate, and sulfide minerals in the siliciclastic bedrock are prone to weathering in near-surface environments where they will affect $\mathrm{pH}$, hardness, alkalinity, sulfate, and associated solutes. Likewise, the chlorite, muscovite, illite, and various other clay minerals readily accommodate ionic substitutions and are widely recognized to be involved in cation-exchange and sorption processes (Hem, 1985; Appelo and Postma, 2005). The glacial lacustrine and alluvial deposits are generally made up of the same constituents as the local bedrock but also may include some minerals from distant locations.

The $\mathrm{pH}, \mathrm{SC}$, and associated constituent concentrations for the Bradford County groundwater samples, classified by the source aquifer bedrock formations, are illustrated as boxplots (fig. 4.4). Of the 72 samples, 47 were obtained from the Lock Haven Formation, 22 from the Catskill Formation, and 3 from the Huntley Mountain Formation. Generally, the medians for $\mathrm{pH}, \mathrm{SC}$, TDS, hardness, and most other constituents were significantly lower and the median dissolved oxygen was significantly higher for the waters from the Huntley Mountain Formation compared to those from the Catskill and Lock Haven Formations. Although median values for $\mathrm{pH}, \mathrm{SC}$, TDS, hardness, $\mathrm{Ca}, \mathrm{Cl}$, and $\mathrm{Br}$ were equivalent for the Catskill and Lock Haven Formations, samples from the Lock Haven Formation had slightly higher median values than the Catskill Formation for $\mathrm{Mg}, \mathrm{Na}$, alkalinity, $\mathrm{SO}_{4}, \mathrm{~F}, \mathrm{~B}, \mathrm{NH}_{3}, \mathrm{Fe}, \mathrm{Mn}$, and methane, and lower median values for $\mathrm{NO}_{3}, \mathrm{U}$, and $\mathrm{Rn}-222$.

\section{Major Ion Compositions Indicated by Trilinear Diagrams}

Trilinear diagrams indicate the percentage contributions of the major cations and anions, in equivalents (molar 
concentration multiplied by ionic charge), relative to the total equivalents for cations and anions in a sample. Although similar in concept to the pie graphs showing ionic conductivity contributions to the SC (fig. 7), the factors used to compute the equivalents do not consider ion size and mobility, which are incorporated with the transport numbers for ionic conductivity (giving somewhat different ionic proportions). The corresponding water type is identified on the basis of the predominant (greater than 50 percent) cation(s) and anion(s) shown on the trilinear diagrams (fig. 8). For the Bradford County well-water samples, calcium/bicarbonate type waters were most abundant, with others classified as sodium/bicarbonate or mixed water types, including calcium-sodium/bicarbonate, calcium-sodium/bicarbonate-chloride, calcium/sulfate-bicarbonate, sodium/bicarbonate-chloride, sodium/bicarbonatesulfate, or sodium/chloride types (fig. 8).

Representative samples are identified on the trilinear diagram in figure 8 in order to explain possible origins of the observed water types. Well-water sample BR 952 has near-neutral $\mathrm{pH}$ and is classified as calcium/bicarbonate type (fig. 8A). A majority of the Bradford County groundwater samples could be classified as calcium/bicarbonate type, which can be produced by the dissolution of calcite $\left(\mathrm{CaCO}_{3}\right)$ by rainwater (recharge) or groundwater. Sample BR 963 is a mixed calcium-sodium/bicarbonate type, whereas sample BR 940 is a sodium/bicarbonate type. Such water types are somewhat common in Bradford County and likely to form by the dissolution of calcite and (or) dolomite combined with cation exchange; these processes are described in more detail below. Samples plotting away from an apex or within the center part of the diagram are classified as mixed hydrochemical types. For example, sample BR 946 is classified as a mixed calcium/sulfate-bicarbonate type, BR 941 is classified as a mixed sodium-calcium/chloride type, and sample BR 921 is a mixed calcium-sodium/chloride-bicarbonate type. The mixed water types imply that multiple sources of constituents or processes may be important. Finally, sample BR 913 was a sodium-chloride type, which is unlike any other sample. The predominance of chloride implies a substantial contribution of salt $\left(\mathrm{NaCl}, \mathrm{CaCl}_{2}\right)$ from road-deicing compounds, sewage or animal waste, or possibly brine of geological origin. Predominant water types or hydrochemical facies (Back, 1966) and evolution pathways for mixing of dilute calcium/bicarbonate $\left(\mathrm{Ca} / \mathrm{HCO}_{3}\right)$ groundwater with road salt, brine, brine combined with cation exchange, or with brine plus calcite dissolution are presented in detail in Gross and Cravotta (2017).

\section{Chloride, Bromide, and Sodium in Groundwater}

The concentrations of chloride $(0.77-1,020 \mathrm{mg} / \mathrm{L})$, bromide (less than $0.01-8.60 \mathrm{mg} / \mathrm{L}$ ), and sodium $(2.18-760 \mathrm{mg} / \mathrm{L})$ for the 72 groundwater samples collected for this study ranged widely and were positively correlated with one another. In Bradford County, chloride concentrations greater than a few milligrams per liter in shallow groundwater may result from human activities or contributions from naturally occurring deeper, more saline groundwater of regional extent. The elevated chloride concentrations may be associated with elevated concentrations of sodium and, in some cases, nitrate and sulfate, which could indicate influence from human or animal waste. Elevated concentrations of chloride also may be associated with elevated (but two or more orders of magnitude smaller) concentrations of bromide, which could indicate influence from residual brine of geologic origin.

Chloride/bromide ratios can be useful to distinguish different sources of chloride (Davis and others, 1998; Whittemore, 2007; Mullaney and others, 2009). Bromide, like chloride, is a soluble anion that exhibits conservative transport properties and can be used as a tracer, if present at detectable concentrations. Some sources of chloride introduced by human activities into the environment, such as salt (sodium chloride) used for road deicing or present in septic effluent, typically have low amounts of bromide and relatively high chloride/ bromide mass ratios. Recent studies of groundwater quality in nearby Susquehanna County in northeastern Pennsylvania (Warner and others, 2012; Llewellyn, 2014; Siegel and others, 2015) have reported groundwater that has concentrations of chloride and chloride/bromide ratios that indicate possible mixing with higher salinity or brine-type waters; these brinetype waters are postulated to be discharging at the surface from undetermined depths below the freshwater aquifer and mixing with shallow, more dilute groundwater.

The chloride/bromide mass ratios for the 72 Bradford County well-water samples are shown in relation to chloride concentrations in figure 9, which also shows curves representing compositions resulting from mixing of different proportions of dilute groundwater with (1) relatively pure sodium chloride $(\mathrm{NaCl})$ salt containing only a trace of bromide (such as salt used for road deicing, in water softeners, and present in human and animal waste), or (2) bromide-rich oil and gas well brines (such as those from wells producing gas from the Marcellus Shale). The majority of Bradford County wellwater samples, including the samples (BR 941 and BR 957) with the second and third highest chloride concentrations (218 and $215 \mathrm{mg} / \mathrm{L}$, respectively) plot on or near the mixing curve for deicing salt, which may enter groundwater from road runoff or septic effluent. However, several well-water samples (BR 913, BR 921, BR 926, BR 907, and BR 910) had relatively high bromide concentrations (greater than $0.4 \mathrm{mg} / \mathrm{L}$ ) with low chloride/bromide mass ratios compared to their chloride concentrations of $50 \mathrm{mg} / \mathrm{L}$ and higher (fig. 9). These wellwater samples plot near or on the mixing curve for oil and gas well brines (fig. 9), suggesting a possible small contribution of chloride from a brine-like source ( 0.02 percent or less). Also plotting along the brine mixing curve is Salt Spring, a naturally occurring saline spring in Susquehanna County.

Of the 8 water samples that had chloride concentrations greater than $20 \mathrm{mg} / \mathrm{L}$ and methane concentrations less than $0.5 \mathrm{mg} / \mathrm{L}, 2$ samples had chloride/bromide ratios that plotted on the road-deicing salt mixing curve, and 4 samples had chloride/bromide ratios that plotted on the brine mixing curve 


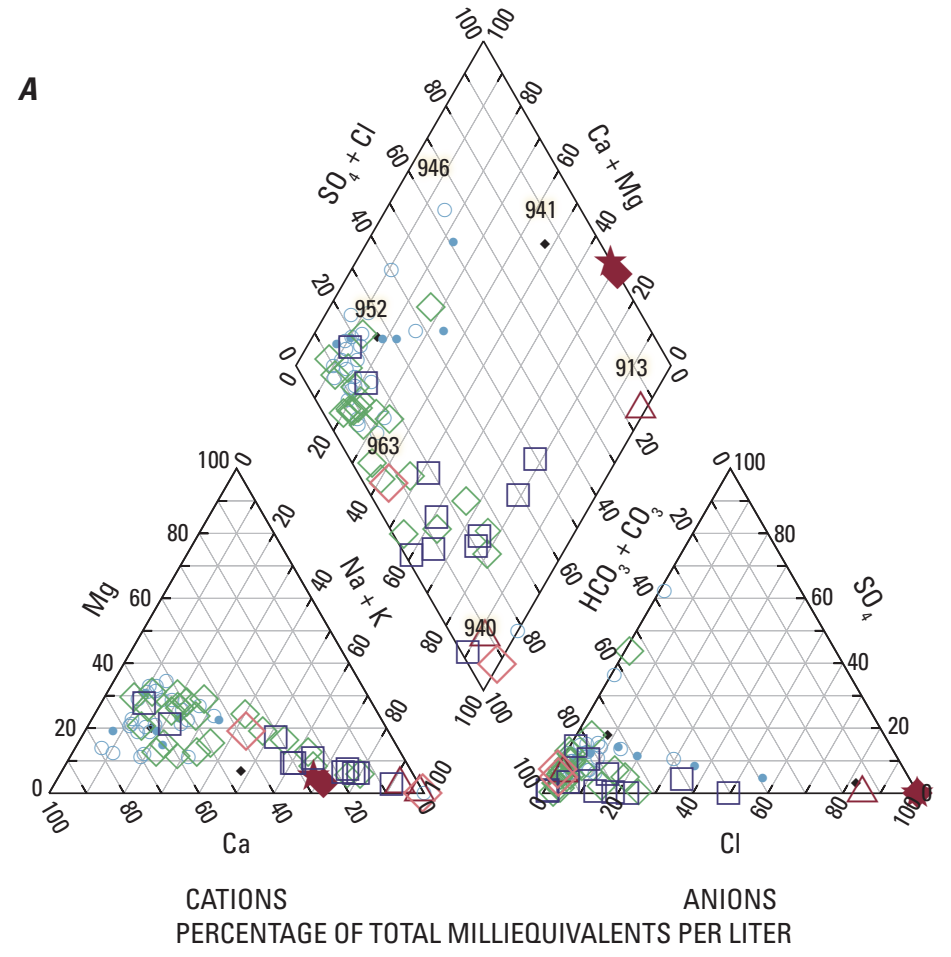

\section{EXPLANATION}

$\mathrm{pH}$

- $6.0<\mathrm{pH}<6.5$

- $6.5<\mathrm{pH}<7.0$

$7.0<\mathrm{pH}<7.5$

$7.5<\mathrm{pH}<8.0$

$\square \quad 8.0<\mathrm{pH}<8.5$

$\triangle 8.5<\mathrm{pH}<9.0$

$>9.0<\mathrm{pH}<9.5$

Chloride $\mathrm{Cl}$

Sodium Na

Sulfate $\mathrm{SO}_{4}$

Magnesium $\mathrm{Mg}$

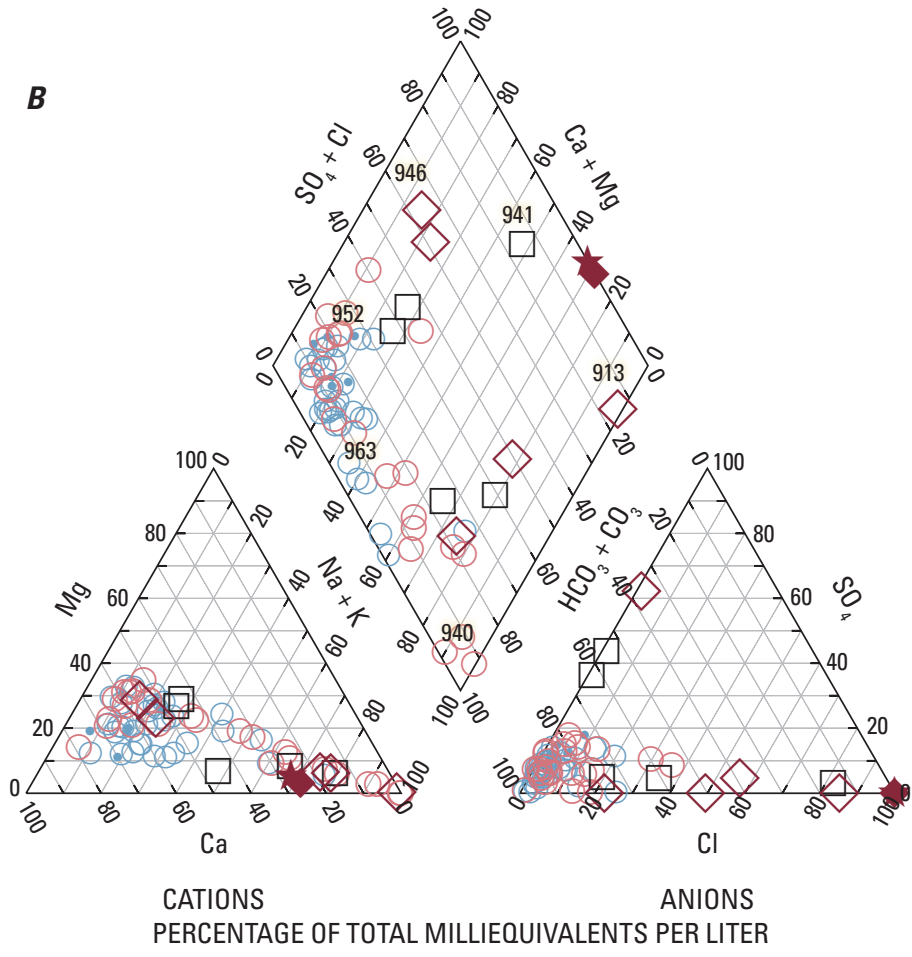

\section{EXPLANATION}

\section{Specific conductance (SC)}

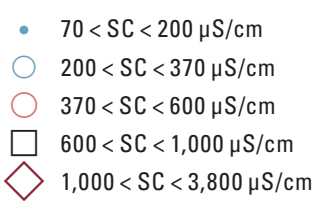

$\star \quad$ Oil and gas brine

Flowback water

41 Domestic well

Carbonate CO

Potassium K

\section{Chemical abbreviations}

Bicarbonate $\mathrm{HCO}_{3}$

Calcium $\mathrm{Ca}$

Carbonate $\mathrm{CO}_{3}$

Chloride $\quad \mathrm{Cl}$

Sodium Na

Sulfate $\mathrm{SO}_{4}$

Magnesium $\mathrm{Mg}$

Potassium K

Figure 8. A, Predominant water types or hydrochemical facies, and $B$, data for 72 groundwater samples from Bradford County, Pennsylvania, 2016, plus median composition of brine from oil and gas wells in western Pennsylvania (Dresel and Rose, 2010) and flowback water from Marcellus Shale gas wells (Hayes, 2009). Symbols for groundwater scaled by $A, \mathrm{pH}$ and $B$, specific conductance (SC). $(<$, less than; $\mu \mathrm{S} / \mathrm{cm}$, microsiemens per centimeter) 
$\boldsymbol{A}$

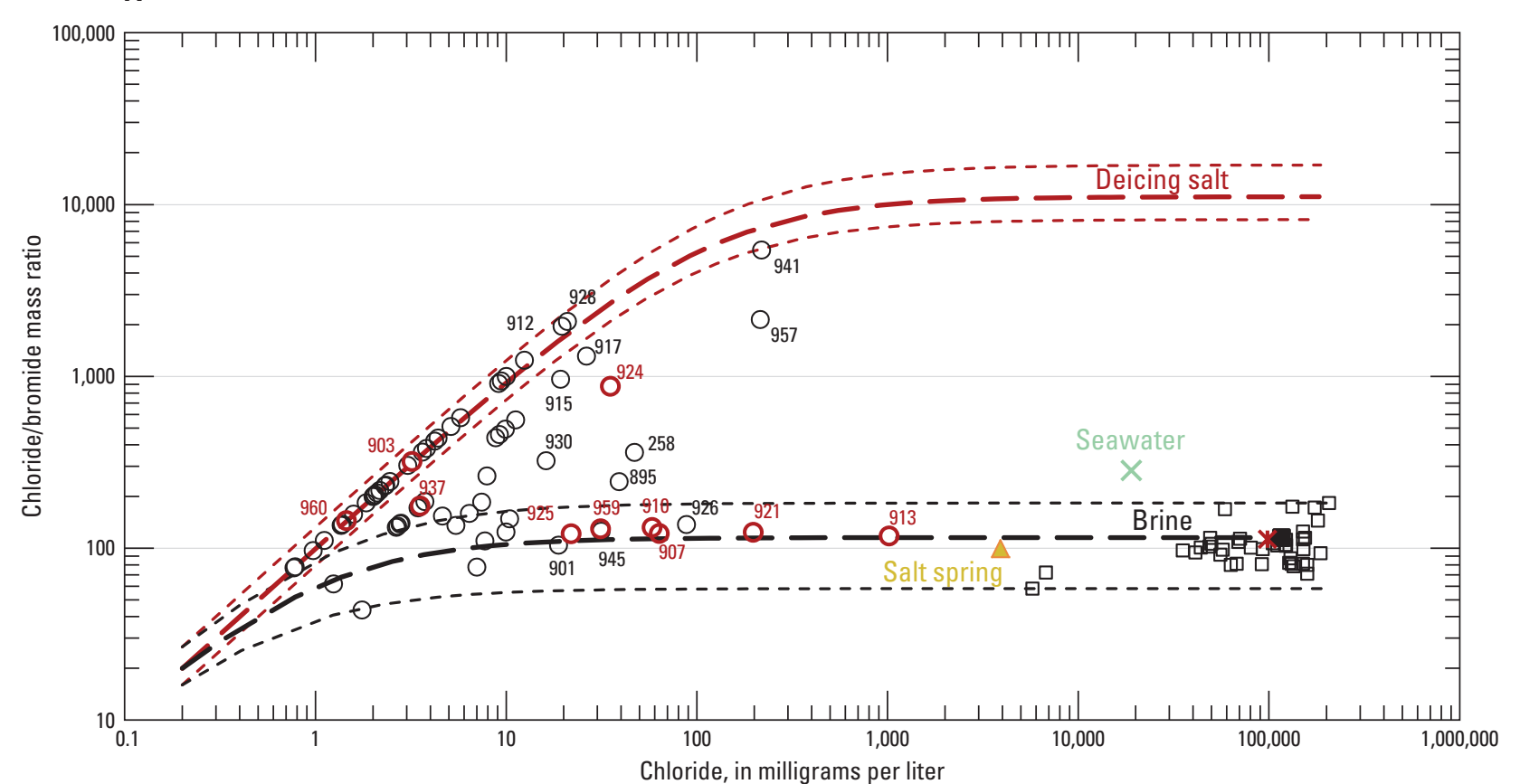

EXPLANATION

\section{- - - Deicing salt mixing curve, upper and lower bounds - Deicing salt mixing curve, median - - Oil and gas brine mixing curve, upper and lower bounds - 0 il and gas brine mixing curve, median}

$\square \quad$ Oil and gas brine (Dresel and Rose, 2010)

- Oil and gas brine, median (Dresel and Rose, 2010)

* Marcellus Shale flowback, median (Hayes, 2009)

$\times \quad$ Seawater, average (Hem, 1985)

$\triangle \quad$ Salt spring (Llewellyn, 2014) $\bigcirc$ Bradford groundwater (methane $<0.5 \mathrm{mg} / \mathrm{L}$ )

Bradford groundwater (methane $\geq 0.5 \mathrm{mg} / \mathrm{L}$ )

Bradford groundwater $(6.0 \leq \mathrm{pH} \leq 6.4)$

O Bradford groundwater $(6.5 \leq \mathrm{pH} \leq 7.4)$

Bradford groundwater $(7.5 \leq \mathrm{pH} \leq 7.9)$

Figure 9. Chloride concentrations compared to $A$, chloride/bromide mass ratios, $\mathrm{B}$, bromide concentrations, and $\mathrm{C}$, sodium concentrations for groundwater from Bradford County, Pennsylvania, 2016, plus median values for Salt Spring (Llewellyn, 2014), flowback waters from Marcellus Shale gas wells (Hayes, 2009), and oil and gas well brines from western Pennsylvania (Dresel and Rose, 2010). Mixing curves computed for initial freshwater with chloride of 0.5 to 0.6 milligrams per liter (mg/L) and bromide of 0.025 to $0.035 \mathrm{mg} / \mathrm{L}$ mixed with road deicing salt having a composition of $\mathrm{NaCl}_{0.99996} \mathrm{Br}_{0.00004}$ (upper curve, after Llewellyn [2014]) or with a median composition of oil and gas well brine (lower curve, after Dresel and Rose [2010]). Selected (numbered) samples have methane concentrations greater than $0.5 \mathrm{mg} / \mathrm{L}$, alkaline $\mathrm{pH}$, or other chemical characteristics that illustrate the variable compositions of the samples from Bradford County. $(<$, less than; $\geq$, greater than or equal to; $\leq$, less than or equal to) 


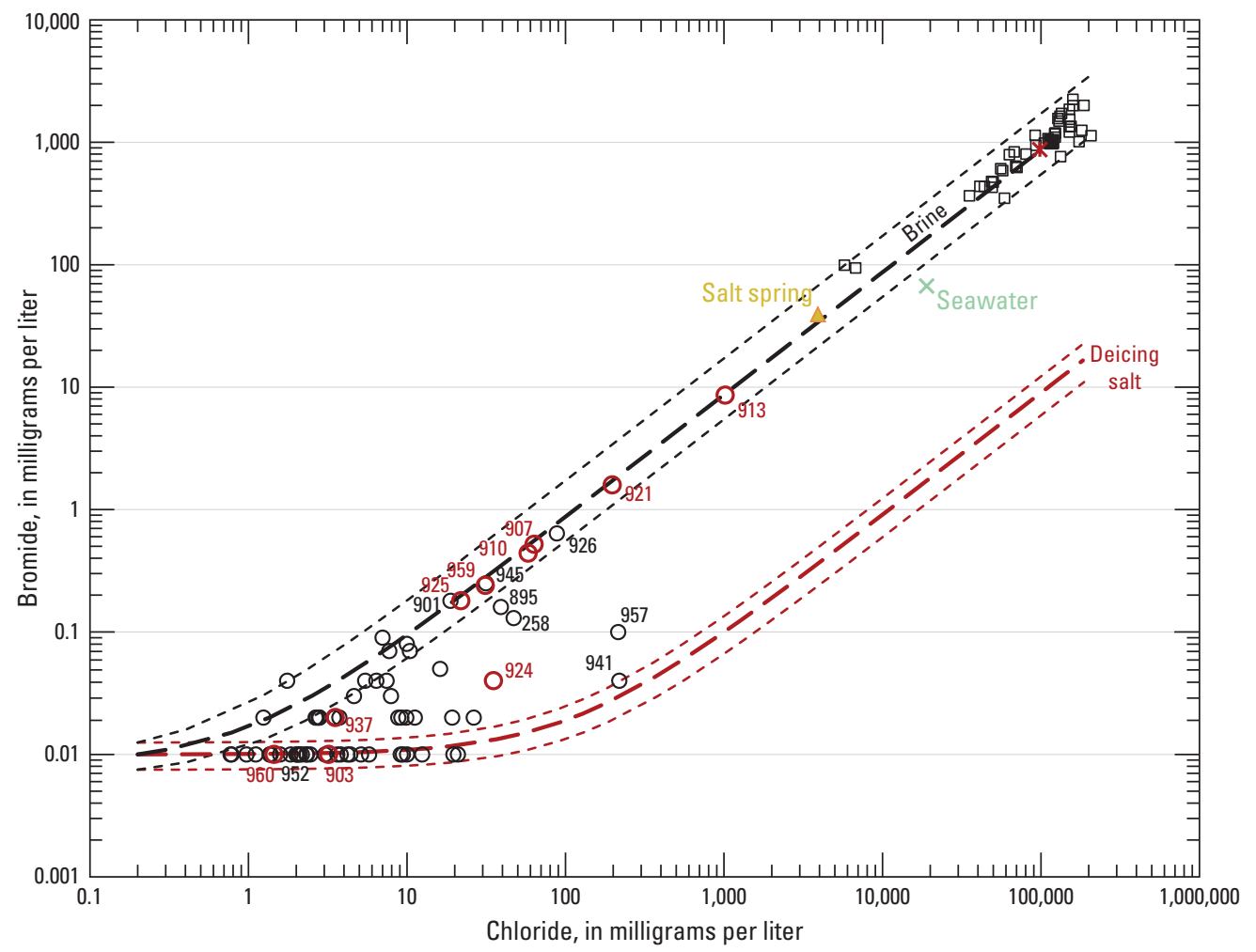

EXPLANATION

\section{- - Deicing salt mixing curve, upper and lower bounds - - Deicing salt mixing curve, median \\ - - Oil and gas brine mixing curve, upper and lower bounds \\ - -0 il and gas brine mixing curve, median}

口 Oil and gas brine (Dresel and Rose, 2010)

- Oil and gas brine, median (Dresel and Rose, 2010)

* Marcellus Shale flowback, median (Hayes, 2009)

$\times$ Seawater, average (Hem, 1985)

$\triangle \quad$ Salt spring (Llewellyn, 2014) $\bigcirc$ Bradford groundwater (methane $<0.5 \mathrm{mg} / \mathrm{L}$ )

○ Bradford groundwater (methane $\geq 0.5 \mathrm{mg} / \mathrm{L}$ )

B Bradford groundwater $(6.0 \leq \mathrm{pH} \leq 6.4)$

Bradford groundwater $(6.5 \leq \mathrm{pH} \leq 7.4)$

Bradford groundwater $(7.5 \leq \mathrm{pH} \leq 7.9)$

Bradford groundwater $(8.0 \leq \mathrm{pH} \leq 9.4)$

Figure 9. Chloride concentrations compared to $A$, chloride/bromide mass ratios, B, bromide concentrations, and C, sodium concentrations for groundwater from Bradford County, Pennsylvania, 2016, plus median values for Salt Spring (Llewellyn, 2014), flowback waters from Marcellus Shale gas wells (Hayes, 2009), and oil and gas well brines from western Pennsylvania (Dresel and Rose, 2010). Mixing curves computed for initial freshwater with chloride of 0.5 to 0.6 milligrams per liter (mg/L) and bromide of 0.025 to $0.035 \mathrm{mg} / \mathrm{L}$ mixed with road deicing salt having a composition of $\mathrm{NaCl}_{0.99996} \mathrm{Br}_{0.00004}$ (upper curve, after Llewellyn [2014]) or with a median composition of oil and gas well brine (lower curve, after Dresel and Rose [2010]). Selected (numbered) samples have methane concentrations greater than $0.5 \mathrm{mg} / \mathrm{L}$, alkaline $\mathrm{pH}$, or other chemical characteristics that illustrate the variable compositions of the samples from Bradford County. $(<$, less than; $\geq$, greater than or equal to; $\leq$, less than or equal to $)-C o n t i n u e d$ 


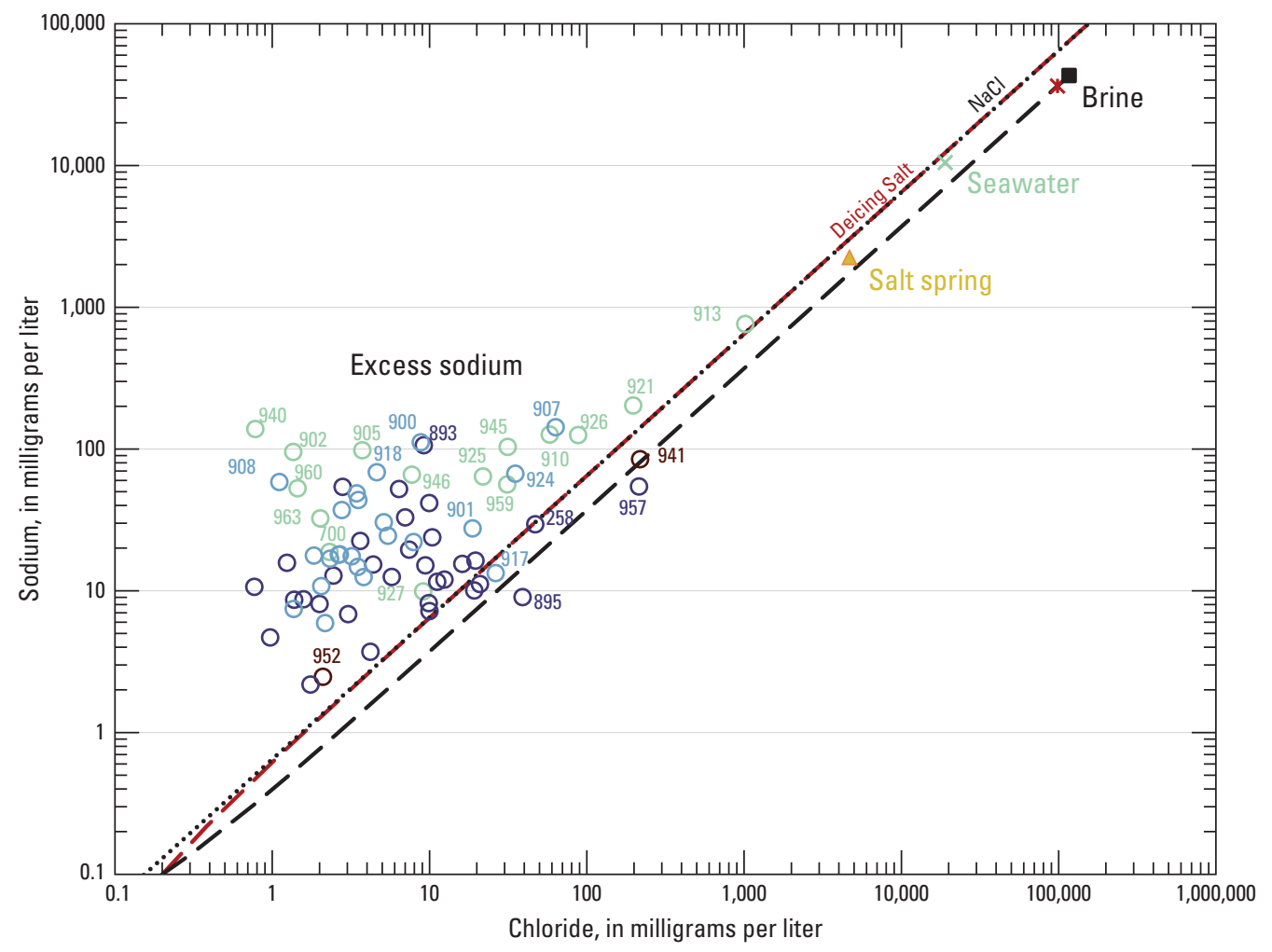

EXPLANATION

\section{- - - Deicing salt mixing curve, upper and lower bounds \\ - Deicing salt mixing curve, median \\ - - - Oil and gas brine mixing curve, upper and lower bounds \\ - -0 il and gas brine mixing curve, median}

Figure 9. Chloride concentrations compared to $A$, chloride/bromide mass ratios, B, bromide concentrations, and C, sodium concentrations for groundwater from Bradford County, Pennsylvania, 2016, plus median values for Salt Spring (Llewellyn, 2014), flowback waters from Marcellus Shale gas wells (Hayes, 2009), and oil and gas well brines from western Pennsylvania (Dresel and Rose, 2010). Mixing curves computed for initial freshwater with chloride of 0.5 to 0.6 milligrams per liter ( $\mathrm{mg} / \mathrm{L}$ ) and bromide of 0.025 to $0.035 \mathrm{mg} / \mathrm{L}$ mixed with road deicing salt having a composition of $\mathrm{NaCl}_{0.99996} \mathrm{Br}_{0.00004}$ (upper curve, after Llewellyn [2014]) or with a median composition of oil and gas well brine (lower curve, after Dresel and Rose [2010]). Selected (numbered) samples have methane concentrations greater than $0.5 \mathrm{mg} / \mathrm{L}$, alkaline $\mathrm{pH}$, or other chemical characteristics that illustrate the variable compositions of the samples from Bradford County. $(<$, less than; $\geq$, greater than or equal to; $\leq$, less than or equal to $)$ Continued 
(fig. 9A-C). Most of these wells were located in valley or valley sides and had depths ranging from 42 to 250 feet below land surface. Nevertheless, many other wells in the valley settings did not exhibit elevated methane concentrations.

Many of the Bradford County groundwater samples had chloride/bromide ratios that plot along the mixing curve for road-deicing salt - which is essentially common salt $(\mathrm{NaCl})$ that is also present in human and animal waste. For example, the water sample from well BR 941, which had the second highest concentration of chloride $(218 \mathrm{mg} / \mathrm{L}=6.15$ millimoles per liter $(\mathrm{mmol} / \mathrm{L}))$, had a similar molar concentration of sodium $(84.6 \mathrm{mg} / \mathrm{L}=3.68 \mathrm{mmol} / \mathrm{L})$, which could result from the dissolution of pure $\mathrm{NaCl}$ by groundwater or recharge water (fig. 9C). Nevertheless, compared to the stoichiometry for $\mathrm{NaCl}$, many other samples that had chloride and bromide compositions plotting on the chloride/bromide mixing curve for salt also contained excess sodium (greater than 1:1 [Na]:[Cl] molar ratio) (fig. 9C). The excess sodium, derived from mineral sources that do not contain chloride, may result from the release of residual sodium on cation-exchange sites on clay minerals to the groundwater.

The chloride/bromide relations for the Bradford County well-water samples collected for this study generally are similar to those for 20 groundwater samples collected in 2012 in Sullivan County (Sloto, 2013), 95 groundwater samples collected in 2012 and 2015 in Pike County (Senior, 2014; Senior and Cravotta, 2017), and 121 groundwater samples collected in 2013-14 in Wayne County (Senior and others, 2017). Although drilling to access natural gas in the Marcellus Shale produces methane and brine, gas wells have not been drilled to this formation in Pike or Wayne Counties, which are within the Delaware River Basin. The regional, localized occurrence of groundwater throughout northeastern and north-central Pennsylvania that has elevated chloride and chloride/bromide ratios that plot along the mixing curve for brine implies a natural origin for most of the observed chloride, bromide, methane, and associated constituents. However, in rare instances, such as well BR 913, the presence of VOCs and other man-made constituents could indicate an anthropogenic source of the observed methane and brine constituents or connectivity with a deep fracture.

\section{Isotopic Composition, Origin, and Spatial Distribution of Methane}

Only the six samples (BR 910, BR 921, BR 903, BR 924, BR 925, and BR 937) with the highest concentrations of methane $(13-77 \mathrm{mg} / \mathrm{L})$ were analyzed for the stable isotopic compositions of methane and the associated concentrations of higher-chain hydrocarbon gases (fig. 10). Ethane $\left(\mathrm{C}_{2} \mathrm{H}_{6}\right)$ was detected at concentrations ranging from 0.01 to $0.13 \mathrm{mg} / \mathrm{L}$ in 4 of 6 samples, but no detectable amount of propane $\left(\mathrm{C}_{3} \mathrm{H}_{8}\right)$ or butane $\left(\mathrm{C}_{4} \mathrm{H}_{10}\right)$ was detected in any samples.

The methane in 5 of these 6 groundwater samples (BR 924, BR 903, BR 910, BR 937, and BR 921) had $\delta^{13} \mathrm{C}_{\mathrm{CH} 4}$ values ranging from -38.23 to -65.05 per mil and $\delta \mathrm{D}_{\mathrm{CH} 4}$ values ranging from -191.2 to -257.2 per mil, which are consistent with the isotopic compositions reported by Baldasarre and others (2014) for mud-gas logging samples from these geologic units. However, the methane in these groundwater samples has lower (lighter) $\delta^{13} \mathrm{C}_{\mathrm{CH} 4}$ values than those reported by Reese and others (2014) for Marcellus Shale methane gas samples from Lycoming County. The sixth sample (BR 925) had a methane concentration of $14 \mathrm{mg} / \mathrm{L}$, with an isotopic composition, $\delta^{13} \mathrm{C}_{\mathrm{CH} 4}$ of -65.1 and $\delta \mathrm{D}_{\mathrm{CH} 4}$ of -257.2 per mil, and is associated with the higher-chain hydrocarbon ethane that is consistent with methane of microbial origin. The ratios of methane to ethane plus higher-chain hydrocarbons $(\mathrm{C} 1 / \mathrm{C} 2+)$ for the other five samples are intermediate between values identified by Reese and others (2014) for microbial or thermogenic methane (fig. 10B). These characteristics could indicate a mixed thermogenic and microbial source (carbondioxide reduction process) of methane and also could imply that methane of microbial origin has been oxidized (becoming isotopically heavier).

Groundwater with relatively elevated methane concentrations (near or above $1 \mathrm{mg} / \mathrm{L}$ ) also had a chemical composition that differed in some respects $(\mathrm{pH}$, selected major ions, and inorganic trace constituents) from groundwater with low methane concentrations. The five groundwater samples with the highest methane concentrations also had among the highest $\mathrm{pH}$ values (greater than 8.2) and elevated concentrations of sodium, lithium, boron, fluoride, arsenic, and bromide. Relatively elevated concentrations of some other constituents, such as barium, strontium, and chloride, commonly were present in, but not limited to, those well-water samples with elevated methane.

Although Llewellyn (2014) described possible associations between elevated methane or brine signatures with deep faulting and associated structural features, the available data for this study indicate that no one physical factor, such as the topographic setting, well depth, or altitude at the bottom of the well, was particularly useful for predicting those well locations with an elevated dissolved concentration of methane. A few parameters stand out as related to topographic position index (TPI) (fig. 4.5); in particular, chloride, bromide, and methane generally increase in the downslope direction. Also, PC4 (table 3.1, Cl-Br) has progressively higher scores in the downslope direction. These general trends are displayed in the conceptual model that indicates discharge of older, deeper groundwater into the valleys (Gross and Cravotta, 2017). 
$\boldsymbol{A}$

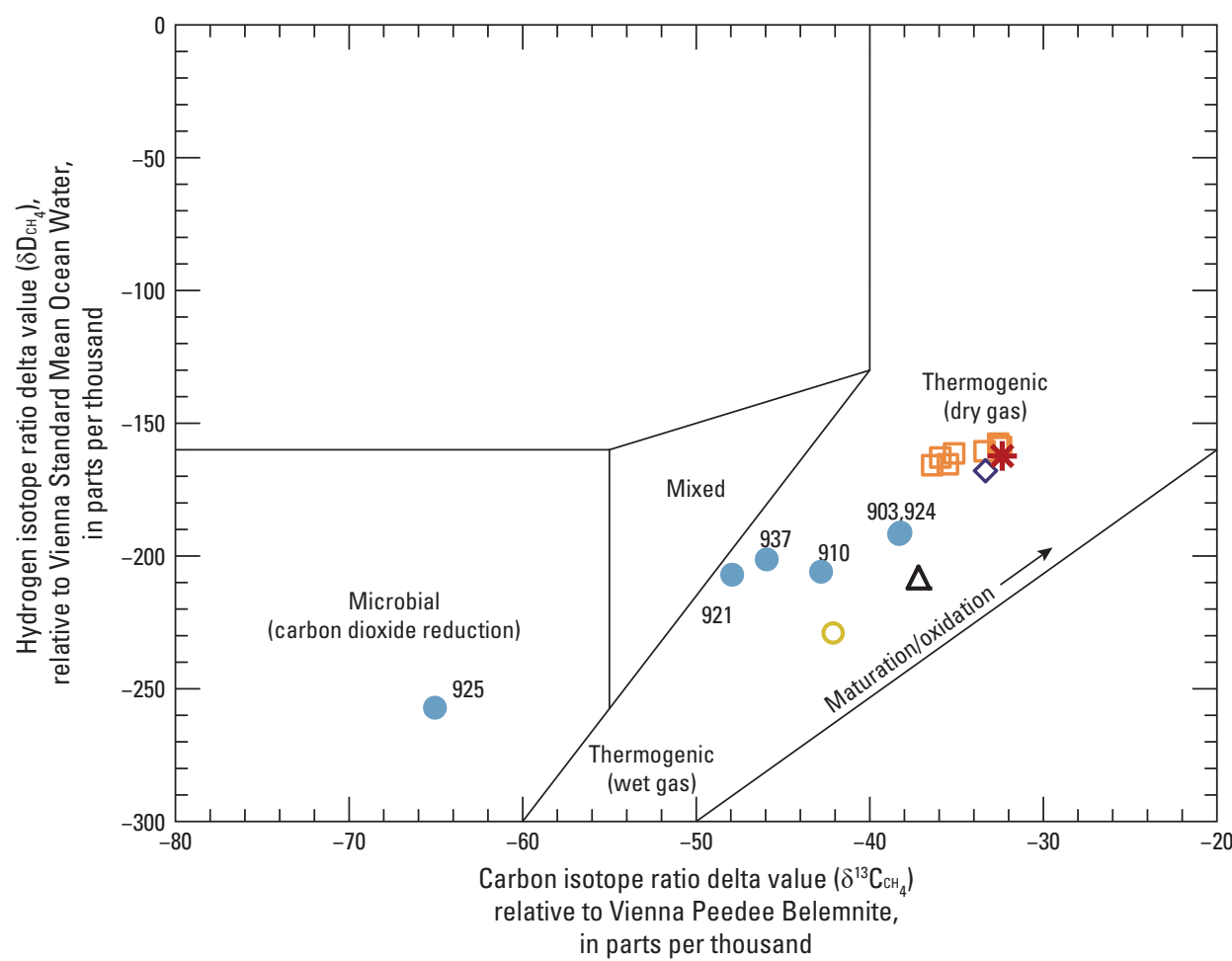

EXPLANATION

Isotopic composition of methane gas in groundwater

Bradford County groundwater, 2016

Mean isotopic composition of methane gas from mud-gas logging of shale-gas wells in Pennsylvania (Baldassare and others, 2014)

Catskill/Lock Haven Formation

A Brallier Formation

$\checkmark$ Hamilton Group

\% Marcellus Shale

Isotopic composition of methane gas Bradford County (Reese and others, 2014)

Marcellus Shale

B

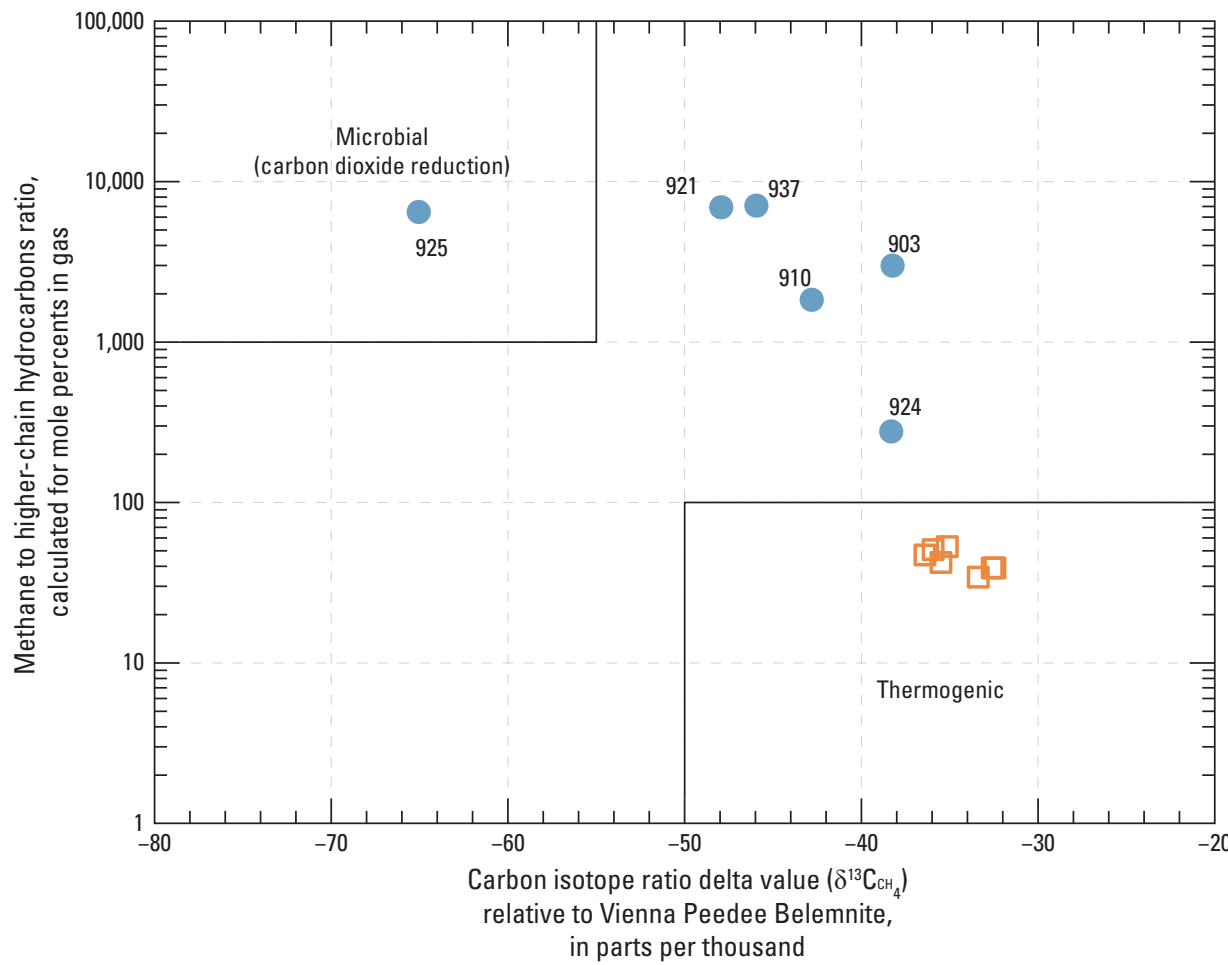

EXPLANATION

Isotopic composition of carbon in methane gas and ratio of methane to higher chain hydrocarbons (C1/C2+)

Bradford County groundwater, 2016

Bradford County Marcellus Shale methane gas samples (Reese and others, 2014)

Figure 10. A, Isotopic composition of methane in groundwater samples collected from five wells in Bradford County, Pennsylvania, 2016; and $B$, ratio of methane to higher-chain hydrocarbons $(C 1 / C 2+)$ in relation to carbon isotopic composition for methane in these samples. Boundaries for microbial and thermogenic gas types and compositional shift related to gas maturation/oxidation, shown by arrow, from Reese and others (2014, fig. 30, p. 38). Marcellus Shale methane gas data for Bradford County from Reese and others (2014, table 7) and mud-gas logging data by geologic formation from Baldassare and others (2014, table 1). 


\section{Summary and Conclusions}

In Bradford County, more than half of the residents use groundwater from private domestic-supply wells as their primary drinking source. The quality of private well water is influenced by the regional and local settings, including the surrounding soil, geology, land use, household plumbing, and well construction. The groundwater used for domestic water supply in Bradford County is obtained primarily from shallow bedrock and from unconsolidated (glacial) deposits that overlie bedrock. Historical land use has been predominately forested, agricultural, and residential, but more recently unconventional oil/gas development has also been distributed throughout the various land uses in the county. Despite legislative attempts, Pennsylvania is one of two states in the Nation without statewide water-well construction standards

To assess the quality of groundwater used for drinking water supplies in Bradford County, samples were collected from 72 domestic wells from May through August 2016 and analyzed for a wide range of constituents that could be evaluated in relation to drinking water health standards, geology, land use, and other environmental factors. Groundwater samples were analyzed for physical and chemical properties, including major ions, nutrients, bacteria, trace elements, volatile organic compounds, ethylene and propylene glycol, alcohols, gross-alpha/beta-particle activity, uranium, radon222 , and dissolved gases. A subset of samples was analyzed for radium isotopes (radium-226 and -228) and for the isotopic composition of methane.

Results of the 2016 study showed that groundwater quality generally met most drinking-water standards. A subset of samples exceeded primary maximum contaminant levels for total coliform bacteria (49.3 percent), Escherichia coli ( 8.5 percent), and arsenic ( 2.8 percent); and secondary maximum contaminant levels for sodium (48.6 percent), manganese ( 30.6 percent), gross alpha and beta activity (16.7 percent), $\mathrm{pH}$ (8.3 percent), iron (11.1 percent), total dissolved solids (5.6 percent), chloride (1.4 percent), and aluminum (1.4 percent). Generally, samples that had elevated TDS, chloride, or arsenic concentrations had high $\mathrm{pH}$. Radon-222 activities exceeded the proposed drinking-water standard of $300 \mathrm{pCi} / \mathrm{L}$ in 70.4 percent of the samples.

The $\mathrm{pH}$ of the groundwater had a large influence on chemical characteristics and ranged from 6.18 to 9.31. Generally, the higher $\mathrm{pH}$ samples had higher potential for elevated concentrations of several constituents, including total dissolved solids, sodium, lithium, chloride, fluoride, boron, arsenic, and methane. For the Bradford County well-water samples, calcium/bicarbonate type waters were the most abundant, with others classified as sodium/bicarbonate or mixed water types, including calcium-sodium/bicarbonate, calciumsodium/bicarbonate-chloride, sodium/bicarbonate-chloride, sodium/bicarbonate-sulfate, or sodium/chloride types. Six principal components $(\mathrm{pH}$, redox, hardness, chloride-bromide, strontium-barium, and molybdenum-arsenic) explain nearly 78.3 percent of the variance in the groundwater dataset.
Groundwater from 12.5 percent of the wells had concentrations of methane greater than the Pennsylvania action level of $7 \mathrm{mg} / \mathrm{L}$; detectable methane concentrations ranged from 0.01 to $77 \mathrm{mg} / \mathrm{L}$. Low levels of ethane (as much as $0.13 \mathrm{mg} / \mathrm{L}$ ) were present in seven samples with the highest methane concentrations. The isotopic composition of methane in five of these groundwater samples was consistent with the isotopic compositions reported for mud-gas logging samples from these geologic units and a thermogenic source of the methane, and a sixth sample suggests the methane in that sample may be of microbial origin. Well-water samples with the higher methane concentrations also had among the higher $\mathrm{pH}$ values and elevated concentrations of sodium, lithium, boron, fluoride, arsenic, and bromide. Relatively elevated concentrations of some other constituents, such as barium and chloride, commonly were present in, but not limited to, those well-water samples with elevated methane.

Four of six groundwater samples with some of the highest methane concentrations had chloride/bromide ratios that indicated mixing with a small amount of brine $(0.02$ percent or less) similar in composition to those reported for gas and oil well brines in Pennsylvania. In several more eastern Pennsylvania counties where gas drilling is absent, groundwater with comparable chloride/bromide ratios and chloride concentrations have been reported, implying a potential natural source of brine. Most of Bradford County well-water samples have chloride concentrations less than $20 \mathrm{mg} / \mathrm{L}$, and those with higher chloride concentrations have chloride/bromide ratios that indicate anthropogenic sources (such as road-deicing salt and septic effluent) or brine. Brines that are naturally present may originate from deeper parts of the aquifer system, whereas anthropogenic sources are more likely to affect shallow groundwater because they occur on or near the land surface.

The available data for this study indicate that no one physical factor such as the topographic setting, well depth, or altitude at the bottom of the well was particularly useful for predicting those well locations with an elevated dissolved concentration of methane. The 2016 assessment of groundwater quality in Bradford County shows groundwater is generally of good quality, but methane and some constituents that occur in high concentration in naturally occurring brine and produced waters from gas and oil wells may be present at low to moderate concentrations in groundwater in various parts of the aquifer. 


\section{References Cited}

American Petroleum Institute/America's Natural Gas Alliance, 2013, Bradford and Susquehanna County, Pennsylvania retrospective case study characterization report: Prepared by Battelle for American Petroleum Institute (API) and America's Natural Gas Alliance (ANGA), Technical Memo $4,77 \mathrm{p}$.

Appelo, C.A.J., and Postma, D., 2005, Geochemistry, groundwater and pollution: Leiden, The Netherlands, A.A. Balkema Publishers, 649 p.

Back, W., 1966, Hydrochemical facies and ground-water flow patterns in northern part of Atlantic Coastal Plain: U.S. Geological Survey Professional Paper 498A, 42 p., accessed October 2017 at https://doi.org/10.3133/pp498A.

Baldassare, F.J., McCaffrey, M.A., and Harper, J.A., 2014, A geochemical context for stray gas investigations in the northern Appalachian Basin-Implications of analyses of natural gases from Neogene-through Devonian-age strata: AAPG Bulletin, v. 98, no. 2, p. 341-372.

Barbash, J.E., and Resek, E.A., 1996, Pesticides in ground water-Distribution, trends, and governing factors: Chelsea Mich., Ann Arbor Press, 598 p.

Belitz, K., Jurgens, B.C., and Johnson, T.D., 2016, Potential corrosivity of untreated groundwater in the United States: U.S. Geological Survey Scientific Investigations Report 2016-5092, 16 p., accessed October 2017 at https://doi. org/10.3133/sir20165092.

Botner, E.C., Townsend-Small, A., Nash, D.B., Xu, X., Schimmelmann, A., and Miller, J.H., 2018, Monitoring concentration and isotopic composition of methane in groundwater in the Utica Shale hydraulic fracturing region of Ohio: Environmental Monitoring and Assessment, v. 190, no. $6,15 \mathrm{p}$.

Boyer, E.W., Swistock, B.R., Clark, J., Madden, M., and Rizzo, D.E., 2012, The impact of Marcellus gas drilling on rural drinking water supplies: The Center for Rural Pennsylvania, 26 p., accessed November 2018 at http://www.rural. palegislature.us/documents/reports/marcellus_and_drinking_water_2012.pdf.

Brown, M.J., and Margolis, S., 2012, Lead in drinking water and human blood lead levels in the United States: U.S. Department of Health and Human Services, Centers for Disease Control and Prevention, accessed November 2018 at https://www.cdc.gov/mmwR/preview/mmwrhtml/su6104a1. htm.
Carter, J.M., Kingsbury, J.A., Hopple, J.A., and Delzer, G.C., 2010 , Concentration data for anthropogenic organic compounds in groundwater, surface water, and finished water of selected community water systems in the United States, 2002-10: U.S. Geological Survey Data Series 544, 36 p. [Also available at https://doi.org/10.3133/ds544.]

Clune, J.W., and Cravotta, C.A. III, 2018, Compilation of data not available in the National Water Information System for domestic wells sampled by the U.S. Geological Survey in Bradford County, Pennsylvania, May-August 2016: U.S. Geological Survey data release, https://doi.org/10.5066/ P9VRV6US.

Commonwealth of Pennsylvania, 2014, The Pennsylvania code chapter 78: Oil and gas wells, accessed October 2016 at http://www.pacode.com/secure/data/025/chapter78/chap78toc.html.

Dai, J., Xia, X., Li, Z., Coleman, D.D., Dias, R.F., Gao, L., Li, J., Deev, A., Li, J., Dessort, D., Duclerc, D., Li, L., Liu, J., Schloemer, S., Zhang, W., Ni, Y., Hu, G., Wang, X., and Tang, Y., 2012, Inter-laboratory calibration of natural gas round robins for $\delta^{2} \mathrm{H}$ and $\delta^{13} \mathrm{C}$ using off-line and on-line techniques: Chemical Geology, v. 310-311, p. 49-55.

Davis, S.N., Whittemore, D.O., and Fabryka-Martin, J., 1998, Uses of chloride/bromide ratios in studies of potable water: Ground Water, v. 36, no. 2, p. 338-350.

DeSimone, L.A., 2009, Quality of water from domestic wells in principal aquifers of the United States, 1991-2004: U. S. Geological Survey Scientific Investigations Report 20085227, 139 p., accessed October 2017 at http://pubs.usgs. gov/sir/2008/5227.

Dresel, P.E., and Rose, A.W., 2010, Chemistry and origin of oil and gas well brines in western Pennsylvania: Pennsylvania Geological Survey, 4th ser, Open-File Report OFOG 10-01.0, 48 p.

Durfor, C.N., and Becker, E., 1964, Public water supplies of the 100 largest cities of the United States, 1962: U.S. Geological Survey Water-Supply Paper 1812, 364 p. [Also available at https://doi.org/10.3133/wsp1812.]

Dzombak, D.A., and Morel, F.M.M., 1990, Surface complexation modeling: hydrous ferric oxide: New York, Wiley Interscience, $393 \mathrm{p}$.

Federal Register, 1999, National primary drinking water regulations; radon-222: Federal Register, v. 64, no. 211, p. 59245-59294, accessed February 2019 at https://www. govinfo.gov/content/pkg/FR-1999-11-02/pdf/99-27741.pdf. 
Fishman, M.J., 1993, Methods of analysis by the U.S. Geological Survey National Water Quality Laboratory; determination of inorganic and organic constituents in water and fluvial sediments: U.S. Geological Survey Open-File Report 93-125, 217 p. [Also available at https://doi.org/10.3133/ ofr93125.]

Fishman, M.J., and Friedman, L.C., 1989, Methods for determination of inorganic substances in water and fluvial sediments: U.S. Geological Survey Techniques of WaterResources Investigations, book 5, chap. A1, 545 p. [Also available at https://doi.org/10.3133/twri05A1.]

Giddings, T., 2014, Water-well construction standards-Past is prologue?: Pennsylvania Council of Professional Geologist (PCPG) Newsletter, no. 3, 3 p., accessed October 2017 at http://www.pcpg.org/Resources/Documents/Newsletters/2014/PCPG\%20Newsletter\%20Q3-2014\%201.pdf.

Gross, E.L., and Cravotta, C.A., 2017, Groundwater quality for 75 domestic wells in Lycoming County, Pennsylvania, 2014: U.S. Geological Survey Scientific Investigations Report 2016-5143, 74 p., accessed October 2017 at https:// doi.org/10.3133/sir20165143.

Hayes, T., 2009, Sampling and analysis of water streams associated with the development of Marcellus Shale gas: Des Plaines, Ill., Report by Gas Technology Institute, for the Marcellus Shale Coalition, accessed October 2017 at http:// www.bucknell.edu/script/environmentalcenter/marcellus/ default.aspx?articleid=14.

Heilweil, V.M., Grieve, P.L., Hynek, S.A., Brantley, S.L., Solomon, D.K., and Risser, D.W., 2015, Stream measurements locate thermogenic methane fluxes in groundwater discharge in an area of shale-gas development: Environmental Science \& Technology, v. 49, no. 7, p. 4057-4065.

Heisig, P.M., and Scott, T.-M., 2013, Occurrence of methane in groundwater of south-central New York State, 2012Systematic evaluation of a glaciated region by hydrogeologic setting: U.S. Geological Survey Scientific Investigations Report 2013-5190, 32 p., accessed February 2019 at https://doi.org/10.3133/sir20135190.

Helsel, D.R., and Hirsch, R.M., 2002, Statistical methods in water resources: U.S. Geological Survey Techniques of Water-Resources Investigations, book 4, chap. A3, 510 p., accessed February 2019 at https://doi.org/10.3133/ twri04A3.

Hem, J.D., 1985, Study and interpretation of the chemical characteristics of natural water: U.S. Geological Survey Water-Supply Paper 2254, 263 p. [Also available at https:// doi.org/10.3133/wsp2254.]
Hirsch, R.M., DeCicco, L., and Lorenz, D., 2015a, dataRetrieval: Retrieval Functions for USGS and EPA hydrologic and water quality data, accessed March 13, 2019, at https://rdrr.io/cran/dataRetrieval/.

Hirsch, R.M., DeCicco, L., and Lorenz, D., 2015b, dataRetrieval reference manual, accessed March 13, 2019, at https://cran.r-project.org/web/packages/dataRetrieval/ dataRetrieval.pdf.

Hirsch, R.M., and De Cicco, L.A., 2015, User guide to Exploration and Graphics for RivEr Trends (EGRET) and dataRetrieval: R packages for hydrologic data (version 2.0, February 2015): U.S. Geological Survey Techniques and Methods, book 4, chap. A10, 93 p. [Also available at https:// doi.org/10.3133/tm4A10.]

Hopke, P.K., Borak, T.B., Doull, J., Cleaver, J.E., Eckerman, K.F., Gundersen, L.C.S., Harley, N.H., Hess, C.T., Kinner, N.E., Kopecky, K.J., McKone, T.E., Sextro, R.G., and Simon, S.L., 2000, Health risks due to radon in drinking water: Environmental Science \& Technology, v. 34, no. 6, p. 921-926.

Jackson, R.B., Vengosh, A., Darrah, T.H., Warner, N.R., Down, A., Poreda, R.J., Osborn, S.G., Zhao, K., and Karr, J.D., 2013, Increased stray gas abundance in a subset of drinking water wells near Marcellus shale gas extraction: Proceedings of the National Academy of Sciences, v. 110, no. 28, p. 11250-11255.

Johnson, T., and Belitz, K., 2017, Domestic well locations and populations served in the contiguous U.S.-1990: Science of the Total Environment, v. 607-608, p. 658-668.

LaRegina, J., 2013, Testimony of the Pennsylvania Council of Professional Geologists before the Committee on Environmental Resources and Energy: Pennsylvania House of Representatives Informational Hearing in House Bill 343, [n.p.], accessed August 31, 2017, at http://wallaby.telicon. com/pa/library/2013/20130417TX.pdf.

Llewellyn, G.T., 2014, Evidence and mechanisms for Appalachian Basin brine migration into shallow aquifers in $\mathrm{NE}$ Pennsylvania, USA: Hydrogeology Journal, v. 22, no. 5, p. 1055-1066.

Llewellyn, G.T., Dorman, F., Westland, J.L., Yoxtheimer, D., Grieve, P., Sowers, T., Humston-Fulmer, E., and Brantley, S.L., 2015, Evaluating a groundwater supply contamination incident attributed to Marcellus Shale gas development: Proceedings of the National Academy of Sciences, v. 112, no. 20, p. 6325-6330.

Lohman, S.W., 1939, Groundwater of north-central Pennsylvania: Pennsylvania Geological Survey, no. 6, 219 p. 
Low, D.J., and Chichester, D.C., 2006, Ground-water-quality data in Pennsylvania-A compilation of computerized [electronic] databases, 1979-2004: U.S. Geological Survey Data Series 150, 22 p., accessed October 2017 at https://doi. org/10.3133/ds 150 .

McCleskey, R.B., Nordstrom, D.K., Ryan, J.N., and Ball, J.W., 2012, A new method of calculating electrical conductivity with applications to natural waters: Geochimica et Cosmochimica Acta, v. 77, p. 369-382.

McMahon, P.B., and Chapelle, F.H., 2008, Redox processes and water quality of selected principal aquifer systems: Groundwater, v. 46, no. 2, p. 259-271, accessed October 16, 2017, at http://onlinelibrary.wiley.com/doi/10.1111/j.17456584.2007.00385.x/abstract.

Miles, C. E., and Whitfield, T. G., comps., 2001, Bedrock geology of Pennsylvania: Pennsylvania Geological Survey, 4th ser., dataset, scale 1:250,000.

Molofsky, L.J., Connor, J.A., Farhat, S.K., Wylie, A.S., Jr., and Wagner, T., 2011, Methane in Pennsylvania water wells unrelated to Marcellus shale fracturing: Oil and Gas Journal, v. 109, no. 49, accessed October 2017 at https:// www.ogj.com/1/vol-109/issue-49/exploration-development/ methane-in-pennsylvania-water-full.html.

Molofsky, L.J., Connor, J.A., Wylie, A.S., Wagner, T., and Farhat, S.K., 2013, Evaluation of methane sources in groundwater in northeastern Pennsylvania: Groundwater, v. 51 , no. 3 , p. 333-349.

Mullaney, J.R., Lorenz, D.L., and Arntson, A.D., 2009, Chloride in groundwater and surface water in areas underlain by the glacial aquifer system, northern United States: U.S. Geological Survey Scientific Investigations Report 2009-5086, 41 p., accessed February 2019 at https://doi. org/10.3133/sir20095086.

Multi-Resolution Land Characteristics Consortium, 2014, National Land Cover Database 2011 land cover: Multi-Resolution Land Characteristics Consortium database, accessed October 15, 2017, at http://www.mrlc.gov.

Murphy, H.M., Thomas, M.K., Schmidt, P.J., Medeiros, D.T., McFadyen, S., and Pintar, K.D.M., 2016, Estimating the burden of acute gastrointestinal illness due to Giardia, Cryptosporidium, Campylobacter, E. coli O157 and norovirus associated with private wells and small water systems in Canada: Epidemiology and Infection, v. 144, no. 07, p. 1355-1370.

National Agriculture Imagery Program (NAIP), 2016, U.S. Department of Agriculture (USDA) Farm Serve Agency (FSA), Salt Lake City Utah, accessed February 2019 at https://gis.apfo.usda.gov/arcgis/rest/services/NAIP.
National Research Council, 1998, Volatile organic compounds (VOCs) - Risk assessment of mixtures of potentially carcinogenic chemicals, chap. 6 in Drinking Water and Health, Selected Issues in Risk Assessment, volume 9: Washington D.C., National Academies Press, p. 162-167.

National Research Council, 1999, Arsenic in drinking water: Washington, D.C., National Academy Press, 273 p.

Osborn, S.G., Vengosh, A., Warner, N.R., and Jackson, R.B., 2011, Methane contamination of drinking water accompanying gas-well drilling and hydraulic fracturing: Proceedings of the National Academy of Sciences, v. 108, no. 20, p. 8172-8176.

Otton, J.K., 1992, The geology of radon: U.S. Geological Survey General Interest Publication, 29 p., accessed October 2017 at https://doi.org/10.3133/7000018.

Parkhurst, D.L., and Appelo, C.A.J., 2013, Description of input and examples for PHREEQC version 3-A computer program for speciation, batch-reaction, one-dimensional transport, and inverse geochemical calculations: U.S. Geological Survey Techniques and Methods, book 6, chap. A43, 497 p., accessed October 2017 at https://doi.org/10.3133/ tm6A43.

Patton, C.J., and Kryskalla, J.R., 2011, Colorimetric determination of nitrate plus nitrite in water by enzymatic reduction, automated discrete analyzer methods: U.S. Geological Survey Techniques and Methods, book 5, chap. B8, 34 p., accessed October 2017 at https://doi.org/10.3133/tm5B8.

Penn State Extension, 2017, Iron and manganese in private water systems: Pennsylvania State University web page, accessed October 23, 2017, at https://extension.psu.edu/ iron-and-manganese-in-private-water-systems.

Pennsylvania Bureau of Topographic and Geologic Survey, 1998, Preliminary landform subdivisions of Pennsylvania, 1998: Pennsylvania Bureau of Topographic and Geologic Survey, Department of Conservation and Natural Resources, digital data, accessed June 23, 2008, at http://www.pasda. psu.edu.

Pennsylvania Bureau of Topographic and Geologic Survey, 2014, Pennsylvania groundwater information system (PaGWIS) documentation: Pennsylvania Bureau of Topographicand Geologic Survey, 4th ser., SQL database, accessed October 2017 from http://www.denr.state.pa.us/ topogeo/groundwater/pagwis/index.htm.

Pennsylvania Department of Environmental Protection, 2017, DEP Office of Oil and Gas Management SPUD data: Pennsylvania Department of Environmental Protection digital database, accessed May 31, 2018, at http://www.depreportingservices.state.pa.us/ReportServer/Pages/ReportViewer. aspx?/Oil_Gas/Spud_External_Data. 
Pennsylvania General Assembly, 2015a, An act amending Title 27 (Environmental Resources) of the Pennsylvania Consolidated Statutes, in conservation and natural resources, providing for water well construction standards: Pennsylvania General Assembly House Bill 81, 8 p., accessed August 31, 2017, at http://www.legis.state.pa.us/cfdocs/billinfo/billinfo. $\mathrm{cfm}$ ? syear $=2015 \& \sin d=0 \&$ body $=$ H\&type $=\mathrm{B} \& b n=0081$.

Pennsylvania General Assembly, 2015b, Proposed well construction and decommissioning standards bill: Pennsylvania General Assembly Senate Bill 653, 7 p., accessed August 31, 2017, at http://www.legis.state.pa.us/cfdocs/ billinfo/BillInfo.cfm?syear=2015\&sind=0\&body=S\&type= $\mathrm{B} \& b n=653$.

Pennsylvania General Assembly, 2016, An act amending Title 27 (Environmental Resources) of the Pennsylvania Consolidated Statutes, in conservation and natural resources, providing for water well construction standards: Pennsylvania General Assembly House Bill 48, 8 p., accessed August 31, 2017, at https:/www.legis.state.pa.us/cfdocs/ billinfo/billinfo.cfm? syear $=2015 \&$ sind $=0 \&$ body $=$ H\&type $=$ $\mathrm{B} \& b n=0048$.

PRISM Group at Oregon State University, 2006a, United States average monthly or annual maximum temperature, 1971-2000: PRISM Group at Oregon State University digital data, accessed March 13, 2019, at http://www.prism. oregonstate.edu/.

PRISM Group at Oregon State University, 2006b, United States average monthly or annual minimum temperature, 1971-2000: PRISM Group at Oregon State University digital data, accessed March 13, 2019, at http://www.prism. oregonstate.edu/.

Raina, P.S., Pollari, F.L., Teare, G.F., and Goss, M.J., 1999, The relationship between E. coli indicator bacteria in wellwater and gastrointestinal illness in rural families: Canadian Journal of Public Health, v. 90, no. 3, p. 172.

Reese, S.O., Neboga, V.V., Pelepko, S., Kosmer, W.J., and Beattie, S., 2014, Groundwater and petroleum resources of Sullivan County, Pennsylvania: Pennsylvania Geological Survey, Water Resource Report 71, 27 p., accessed February 2019 at http://maps.denr.pa.gov/publications/Default. aspx?id=799.

Reilly, D., Singer, D., Jefferson, A., and Eckstein, Y., 2015, Identification of local groundwater pollution in northeastern Pennsylvania-Marcellus flowback or not?: Environmental Earth Sciences, v. 73, no. 12, p. 8097-8109.

Risser, D.W., Thompson, R.E., and Stuckey, M.H., 2008, Regression method for estimating long-term mean annual ground-water recharge rates from base flow in Pennsylvania: U.S. Geological Survey Scientific Investigations Report 2008-5185, 23 p., accessed October 2017 at https://pubs. er.usgs.gov/publication/sir20085185.
SAS Institute, Inc., 2012, SAS 9.4 for Windows: Cary, N.C., USA, SAS Institute, Inc.

Scott, J.C., 1990, Computerized stratified random site-selection approaches for design of a ground-water-quality sampling network: U.S. Geological Survey Water-Resources Investigations Report 90-4101, 109 p., accessed January 1, 1994, at https://doi.org/10.3133/wri904101.

Senior, L.A., 2009, Groundwater-quality assessment, Pike County, Pennsylvania, 2007: U.S. Geological Survey Scientific Investigations Report 2009-5129, 53 p., accessed October 2017 at https://doi.org/10.3133/sir20095129.

Senior, L.A., 2014, A reconnaissance spatial and temporal baseline assessment of methane and inorganic constituents in groundwater in bedrock aquifers, Pike County, Pennsylvania, 2012-2013: U.S. Geological Survey Scientific Investigations Report 2014-5117, 91 p., accessed November 9, 2018, at https://pubs.er.usgs.gov/publication/sir20145117.

Senior, L.A., Cravotta, C.A., and Sloto, R.A., 2017, Baseline assessment of groundwater quality in Wayne County, Pennsylvania, 2014: U.S. Geological Survey Scientific Investigations Report 2016-5073, 152 p., accessed November 2018 at https://doi.org/10.3133/sir20165073.

Senior, L.A., and Cravotta III, C.A., 2017, Baseline assessment of groundwater quality in Pike County, Pennsylvania, 2015: U.S. Geological Survey Scientific Investigations Report 2017-5110, accessed November 9, 2018, at https:// doi.org/10.3133/sir20175110.

Sevon, W.D., and Braun D.D., 1997, Glacial Deposits of Pennsylvania, Map 59: Pennsylvania Bureau of Topographic and Geologic Survey, Department of Conservation and Natural Resources, accessed November 11, 2011, at https://www. nrc.gov/docs/ML0707/ML070720645.pdf.

Sevon, W. D., 2000, Physiographic provinces of Pennsylvania: Pennsylvania Geological Survey, 4th ser., Map 13, scale $1: 2,000,000$.

Siegel, D.I., Smith, B., Perry, E., Bothun, R., and Hollingsworth, M., 2015, Pre-drilling water-quality data of groundwater prior to shale gas drilling in the Appalachian BasinAnalysis of the Chesapeake Energy Corporation dataset: Applied Geochemistry, v. 63, no. C, p. 37-57.

Simpson, H., 2004, Promoting the management and protection of private water wells: Journal of Toxicology and Environmental Health, part A, v. 67, no. 20-22, p. 1679-1704.

Sloto, R.A., 2013, Baseline groundwater quality from 20 domestic wells in Sullivan County, Pennsylvania, 2012: U.S. Geological Survey Scientific Investigations Report 2013-5085, 30 p., accessed October 2017 at https://doi. org/10.3133/sir20135085. 
Smedley, P., and Kinniburgh, D., 2002, A review of the source, behaviour and distribution of arsenic in natural waters: Applied Geochemistry, v. 17, no. 5, p. 517-568.

Snoeyink, V.L., and Jenkins, D., 1981, Water chemistry: New York, John Wiley \& Sons, $463 \mathrm{p}$.

Soeder, D.J., 2017, Unconventional-The development of natural gas from the Marcellus Shale: The Geological Society of America Special Paper 527, 143 p.

Swistock, B.R., 2012, Statement of the senior water resources extension associate of the School of Forest Resources, College of Agricultural Sciences, The Pennsylvania State University before the Consumer Affairs Committee: Pennsylvania House of Representatives Informational Hearing in House Bill 1855, 4 p., accessed August 31, 2017, at http:// wallaby.telicon.com/pa/library/2012/20120110tq.pdf.

Swistock, B.R., Clemens, S., and Sharpe, W.E., 2009, Drinking water quality in rural Pennsylvania and the effects of management practices: The Center for Rural Pennsylvania, $23 \mathrm{p}$.

Swistock, B.R., and Sharpe, W.E., 2005, The influence of well construction on bacterial contamination of private water wells in Pennsylvania: Journal of Environmental Health, v. 68 , no. 2 , p. 17.

Swistock, B.R., and Sharpe, W.E., 2006, Methane gas and its removal from wells in Pennsylvania: Pennsylvania State University Cooperative Extension Water Facts \#24, accessed November 2018 at https://extension.psu.edu/methane-gas-and-its-removal-from-water-wells.

Swistock, B.R., Sharpe, W.E., and Robillard, P.D., 1993, A survey of lead, nitrate and radon contamination of private individual water systems in Pennsylvania: Journal of Environmental Health, v. 55, no. 5, p. 6.

Taylor, L.E., Werkheiser, W.H., and Kriz, M.L., 1983, Groundwater resources of the West Branch Susquehanna River Basin, Pennsylvania: Pennsylvania Geological Survey, 4th ser, $143 \mathrm{p}$.

U.S. Census Bureau, 2011, Census 2000 \& 2010 redistricting data (Public Law 94-171) summary file: Prepared by The Pennsylvania State Data Center, Middletown, Pa., accessed October 15, 2017, at http://pasdc.hbg.psu.edu/sdc/pasdc files/census2010/032011_release/MCD_PA_PL-2010-2000_ Total-Population.xls.

U.S. Environmental Protection Agency, 2001, Total coliform rule-A quick reference guide: U.S. Environmental Protection Agency, Office of Water, EPA 816-F-010035, 2 p.

U.S. Environmental Protection Agency, 2003, Drinking water advisory - Consumer acceptability advice and health effects analysis on sodium: U.S. Environmental Protection Agency EPA 822-R-03-006, 29 p.
U.S. Environmental Protection Agency, 2012a, Edition of the drinking water standards and health advisories: U.S. Environmental Protection Agency EPA 822-S-12-001, accessed October 15, 2017, at http://water.epa.gov/action/advisories/ drinking/upload/dwstandards2012.pdf.

U.S. Environmental Protection Agency, 2012b, Study of the potential impacts of hydraulic fracturing on drinking water resources-Progress report: U.S. Environmental Protection Agency, EPA/601/R-12/011, 262 p.

U.S. Geological Survey, variously dated, National field manual for the collection of water-quality data: U.S. Geological Survey Techniques of Water-Resources Investigations, book 9, chaps. A1-A7, accessed October 2017 at http:// pubs.water.usgs.gov/twri9A.

U.S. Geological Survey, 2009, 1-Arc second National Elevation Dataset: U.S. Geological Survey, SDE raster digital data, accessed October 2017 at https://lta.cr.usgs.gov/NED.

U.S. Geological Survey, 2014, NLCD 2011 land cover (2011 edition): U.S. Geological Survey, remote-sensing image, accessed October 2018 at http://www.mrlc.gov.

U.S. Geological Survey, 2018, Water Quality Service: U.S. Geological Survey Water Services web page, accessed November 9, 2018, at https://waterservices.usgs.gov/.

Velleman, P.F., and Hoaglin, D., 1981, Applications, basics, and computing of exploratory data analysis: Boston, Mass., Duxbury Press, 354 p.

Vidic, R.D., Brantley, S.L., Vandenbossche, J.M., Yoxtheimer, D., and Abad, J.D., 2013, Impact of shale gas development on regional water quality: Science, v. 340, no. 6134, 10 p.

Wagner, D.R., 2012, Testimony of the Pennsylvania Council of Professional Geologists chair of Government Affairs Committee before the Consumer Affairs Committee: Pennsylvania House of Representatives Informational Hearing in House Bill 1855, [n.p.], accessed August 31, 2017, at http://www.pcpg.org/Resources/Documents/PCPG\%20 HB1855\%20Testimony\%20with\%20Recommendations\%20 2012-1-5.pdf.

Warner, K.L., and Ayotte, J.D., 2015, The quality of our Nation's waters - Water quality in the glacial aquifer system, northern United States, 1993-2009: U.S. Geological Survey Circular 1352, 128 p., accessed October 2017 at https://doi.org/10.3133/cir1352.

Warner, N.R., Jackson, R.B., Darrah, T.H., Osborn, S.G., Down, A., Zhao, K., White, A., and Vengosh, A., 2012, Geochemical evidence for possible natural migration of Marcellus Formation brine to shallow aquifers in Pennsylvania: Proceedings of the National Academy of Sciences, v. 109 , no. 30, p. 11961-11966. 
Wen, T., Niu, X., Gonzales, M., Zheng, G., Li, Z., and Brantley, S.L., 2018, Big groundwater data sets reveal possible rare contamination amid otherwise improved water quality for some analytes in a region of Marcellus Shale development: Environmental Science \& Technology, v. 52, no. 12 , p. 7149-7159.

Weston Solutions, 2012, Evaluation of geology and water well data associated with the EPA hydraulic fracturing retrospective case study Bradford County, Pennsylvania: Prepared by Weston Solutions for Chesapeake Energy, 360 p., accessed October 2017 at https://www.chk.com/news/documents/20120529_chk_westonstudy_bradfordpa.pdf.

Whittemore, D.O., 2007, Fate and identification of oil-brine contamination in different hydrogeologic settings: Applied Geochemistry, v. 22, no. 10, p. 2099-2114.

Williams, J.H., Taylor, L.E., and Low, D.J., 1998, Hydrogeology and groundwater quality of the glaciated valleys of Bradford, Tioga, and Potter Counties, Pennsylvania: Pennsylvania Geological Survey, Water Resource Report $68,89 \mathrm{p}$.

Yager, R.M., McCoy, K.J., Voss, C.I., Sanford, W.E., and Winston, R.B., 2017, The role of uplift and erosion in the persistence of saline groundwater in the shallow subsurface: Geophysical Research Letters, v. 44, no. 8, p. 3672-3681.

Zimmerman, T.M., Zimmerman, M.L., and Lindsey, B.D., 2001, Relation between selected well-construction characteristics and occurrence of bacteria in private householdsupply wells, south-central and southeastern Pennsylvania: U.S. Geological Survey Water-Resources Investigations Report 2001-4206, 22 p., accessed October 2018 at https:// doi.org/10.3133/wri014206.

Zogorski, J.S., Carter, J.M., Ivahnenko, T., Lapham, W.W., Moran, M.J., Rowe, B.L., Squillace, P.J., and Toccalino, P.L., 2006, Volatile organic compounds in the nation's ground water and drinking-water supply wells: U.S. Geological Survey Circular 1292, 101 p., accessed October 2018 at https://pubs.er.usgs.gov/publication/cir1292. 

Appendixes 1-4 



\section{Appendix 1.}

Table 1.1. Compilation of data not available in the National Water Information System, including station name, station identification number, lithologic class, and topographic position index for domestic wells sampled by the U.S. Geological Survey in Bradford County, Pennsylvania, May-August 2016 (available online as an Excel file at https://doi.org/10.5066/P9VRV6US).

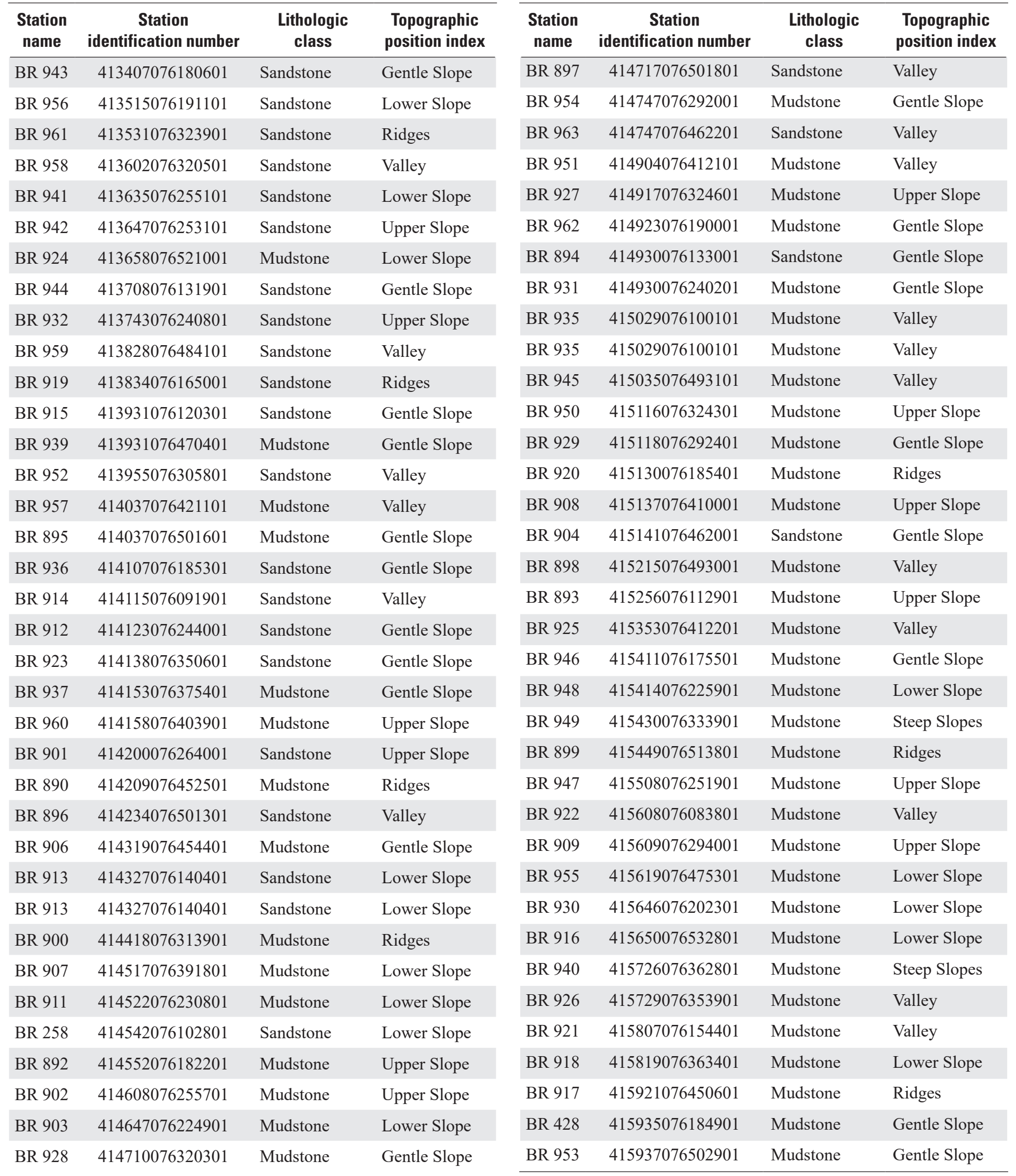




\section{Appendix 2.}

Table 2.1. Minimum, median, and maximum values of selected characteristics and constituents in groundwater samples collected from 72 domestic wells in Bradford County, Pennsylvania, May-August 2016. Available U.S. Environmental Protection Agency Maximum Contaminant Levels, Action Levels, and Secondary Maximum Contaminant Levels for analyzed constituents (2012 Edition of the Drinking Water Standards and Health Advisories [EPA 822-S-12-001]).

[n, number of results; EPA, U.S. Environmental Protection Agency; MCL, Maximum Contaminant Level; SMCL, Secondary Maximum Contaminant Level; $\mu \mathrm{g} / \mathrm{L}$, micrograms per liter; $<$, less than; - , no MCL, Action Level, or SMCL established]

\begin{tabular}{|c|c|c|c|c|c|c|c|c|c|c|c|}
\hline \multirow{2}{*}{ Constituent (units) } & \multirow{2}{*}{$\mathbf{n}$} & \multirow{2}{*}{ Minimum } & \multirow{2}{*}{ Median } & \multirow{2}{*}{ Maximum } & \multicolumn{2}{|c|}{$\begin{array}{l}\text { Results above the } \\
\text { reporting level }\end{array}$} & \multicolumn{3}{|c|}{ Results exceeding standard } & \multirow{2}{*}{$\begin{array}{l}\text { EPA } \\
\text { MCL }\end{array}$} & \multirow{2}{*}{$\begin{array}{l}\text { EPA } \\
\text { SMCL }\end{array}$} \\
\hline & & & & & Number & Percent & Number & Percent & Graph & & \\
\hline \multicolumn{12}{|c|}{ Volatile Organic Compounds } \\
\hline 1,1,1,2-Tetrachloroethane $(\mu \mathrm{g} / \mathrm{L})$ & 72 & $<0.5$ & $<0.5$ & $<0.5$ & 0 & 0.00 & - & - & - & - & - \\
\hline 1,1,1-Trichloroethane $(\mu \mathrm{g} / \mathrm{L})$ & 72 & $<0.5$ & $<0.5$ & $<0.5$ & 0 & 0.00 & 0 & 0 & & 200 & - \\
\hline 1,1,2-Trichloroethane $(\mu \mathrm{g} / \mathrm{L})$ & 72 & $<0.5$ & $<0.5$ & $<0.5$ & 0 & 0.00 & 0 & 0 & & 5 & - \\
\hline 1,1-Dichloroethane $(\mu \mathrm{g} / \mathrm{L})$ & 72 & $<0.5$ & $<0.5$ & $<0.5$ & 0 & 0.00 & - & - & - & - & - \\
\hline 1,1-Dichloroethene (1,1-Dichloroethylene) ( $\mu \mathrm{g} / \mathrm{L})$ & 72 & $<0.5$ & $<0.5$ & $<0.5$ & 0 & 0.00 & 0 & 0 & 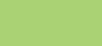 & 7 & - \\
\hline 1,1-Dichloropropene $(\mu \mathrm{g} / \mathrm{L})$ & 72 & $<0.5$ & $<0.5$ & $<0.5$ & 0 & 0.00 & - & - & - & - & - \\
\hline 1,2,4-Trichlorobenzene $(\mu \mathrm{g} / \mathrm{L})$ & 72 & $<0.5$ & $<0.5$ & $<0.5$ & 0 & 0.00 & 0 & 0 & . & 70 & - \\
\hline $1,2,4$-Trimethylbenzene $(\mu \mathrm{g} / \mathrm{L})$ & 72 & $<0.5$ & $<0.5$ & $<0.5$ & 0 & 0.00 & - & - & - & - & - \\
\hline 1,2-Dichlorobenzene (o-Dichlorobenzene) $(\mu \mathrm{g} / \mathrm{L}$ ) & 72 & $<0.5$ & $<0.5$ & $<0.5$ & 0 & 0.00 & 0 & 0 & 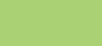 & 600 & - \\
\hline 1,2-Dichloroethane $(\mu \mathrm{g} / \mathrm{L})$ & 72 & $<0.5$ & $<0.5$ & $<0.5$ & 0 & 0.00 & 0 & 0 & 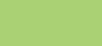 & 5 & - \\
\hline 1,2-Dichloropropane $(\mu \mathrm{g} / \mathrm{L})$ & 72 & $<0.5$ & $<0.5$ & $<0.5$ & 0 & 0.00 & 0 & 0 & & 5 & - \\
\hline $1,3,5$-Trimethylbenzene $(\mu \mathrm{g} / \mathrm{L})$ & 72 & $<0.5$ & $<0.5$ & $<0.5$ & 0 & 0.00 & - & - & - & - & - \\
\hline 1,3-Dichlorobenzene (m-Dichlorobenzene) $(\mu \mathrm{g} / \mathrm{L})$ & 72 & $<0.5$ & $<0.5$ & $<0.5$ & 0 & 0.00 & - & - & - & - & - \\
\hline 1,3-Dichloropropane $(\mu \mathrm{g} / \mathrm{L})$ & 72 & $<0.5$ & $<0.5$ & $<0.5$ & 0 & 0.00 & - & - & - & - & - \\
\hline Acetone $(\mu \mathrm{g} / \mathrm{L})$ & 72 & $<1.0$ & $<1.0$ & $<1.0$ & 0 & 0.00 & - & - & - & - & - \\
\hline Benzene $(\mu \mathrm{g} / \mathrm{L})$ & 72 & $<0.5$ & $<0.5$ & $<0.5$ & 0 & 0.00 & 0 & 0 & 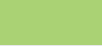 & 5 & - \\
\hline Bromobenzene $(\mu \mathrm{g} / \mathrm{L})$ & 72 & $<0.5$ & $<0.5$ & $<0.5$ & 0 & 0.00 & - & - & - & - & - \\
\hline Bromochloromethane $(\mu \mathrm{g} / \mathrm{L})$ & 72 & $<0.5$ & $<0.5$ & $<0.5$ & 0 & 0.00 & - & - & - & - & - \\
\hline Bromomethane (Methyl bromide) $(\mu \mathrm{g} / \mathrm{L})$ & 72 & $<0.5$ & $<0.5$ & $<0.5$ & 0 & 0.00 & - & - & - & - & - \\
\hline Carbon Disulfide $(\mu \mathrm{g} / \mathrm{L})$ & 72 & $<0.5$ & $<0.5$ & $<0.5$ & 0 & 0.00 & - & - & - & - & - \\
\hline Chlorobenzene $(\mu \mathrm{g} / \mathrm{L})$ & 72 & $<0.5$ & $<0.5$ & $<0.5$ & 0 & 0.00 & 0 & 0 & & 100 & - \\
\hline $\mathrm{CHBrCl} 2, \mathrm{wu}, \mu \mathrm{g} / \mathrm{L}$ (32101) & 72 & $<0.5$ & $<0.5$ & 1.04 & 1 & 1.39 & 0 & 0 & . & - & 80 \\
\hline Chloroethane ( $\mu \mathrm{g} / \mathrm{L})$ & 72 & $<0.5$ & $<0.5$ & $<0.5$ & 0 & 0.00 & - & - & - & - & - \\
\hline
\end{tabular}


Table 2.1. Minimum, median, and maximum values of selected characteristics and constituents in groundwater samples collected from 72 domestic wells in Bradford County, Pennsylvania, May-August 2016. Available U.S. Environmental Protection Agency Maximum Contaminant Levels, Action Levels, and Secondary Maximum Contaminant Levels for analyzed constituents (2012 Edition of the Drinking Water Standards and Health Advisories [EPA 822-S-12-001]).-Continued

\begin{tabular}{|c|c|c|c|c|c|c|c|c|c|c|c|}
\hline \multirow{2}{*}{ Constituent (units) } & \multirow{2}{*}{$\mathbf{n}$} & \multirow{2}{*}{ Minimum } & \multirow{2}{*}{ Median } & \multirow{2}{*}{ Maximum } & \multicolumn{2}{|c|}{$\begin{array}{l}\text { Results above the } \\
\text { reporting level }\end{array}$} & \multicolumn{3}{|c|}{ Results exceeding standard } & \multirow{2}{*}{$\begin{array}{l}\text { EPA } \\
\text { MCL }\end{array}$} & \multirow{2}{*}{$\begin{array}{l}\text { EPA } \\
\text { SMCL }\end{array}$} \\
\hline & & & & & Number & Percent & Number & Percent & Graph & & \\
\hline \multicolumn{12}{|c|}{ Volatile Organic Compounds-Continued } \\
\hline Chloromethane, wu, $\mu \mathrm{g} / \mathrm{L}$ (34418) & 72 & $<0.5$ & $<0.5$ & $<0.5$ & 0 & 0.00 & - & - & - & - & - \\
\hline cis-1,2-dichloroethene $(\mu \mathrm{g} / \mathrm{L})$ & 72 & $<0.5$ & $<0.5$ & $<0.5$ & 0 & 0.00 & 0 & 0 & & 70 & - \\
\hline cis-1,3-dichloropropene ( $\mu \mathrm{g} / \mathrm{L})$ & 72 & $<0.5$ & $<0.5$ & $<0.5$ & 0 & 0.00 & - & - & - & - & - \\
\hline Dibromochloromethane $(\mu \mathrm{g} / \mathrm{L})$ & 72 & $<0.5$ & $<0.5$ & 1.58 & 1 & 1.39 & 0 & 0 & & 80 & - \\
\hline Dibromomethane $(\mu \mathrm{g} / \mathrm{L})$ & 72 & $<0.5$ & $<0.5$ & $<0.5$ & 0 & 0.00 & - & - & - & - & - \\
\hline Ethylbenzene $(\mu \mathrm{g} / \mathrm{L})$ & 72 & $<0.5$ & $<0.5$ & $<0.5$ & 0 & 0.00 & 0 & 0 & & 700 & - \\
\hline Hexachlorobutadiene (1,3-Hexachlorobutadiene) $(\mu \mathrm{g} / \mathrm{L})$ & 72 & $<0.5$ & $<0.5$ & $<0.5$ & 0 & 0.00 & - & - & - & - & - \\
\hline Iodomethane (Methyl iodide) $(\mu \mathrm{g} / \mathrm{L})$ & 72 & $<0.5$ & $<0.5$ & $<0.5$ & 0 & 0.00 & - & - & - & - & - \\
\hline Isopropylbenzene (Cumene) $(\mu \mathrm{g} / \mathrm{L})$ & 72 & $<0.5$ & $<0.5$ & $<0.5$ & 0 & 0.00 & - & - & - & - & - \\
\hline$m$-Xylene plus $p$-xylene $(\mu \mathrm{g} / \mathrm{L})$ & 71 & $<1.0$ & $<1.0$ & $<1.0$ & 0 & 0.00 & 0 & 0 & & 10,000 & - \\
\hline Methyl chloride (Chloromethane) $(\mu \mathrm{g} / \mathrm{L})$ & 72 & $<0.5$ & $<0.5$ & $<0.5$ & 0 & 0.00 & - & - & - & - & - \\
\hline Methyl tert-butyl ether (MTBE) $(\mu \mathrm{g} / \mathrm{L})$ & 72 & $<0.5$ & $<0.5$ & $<0.5$ & 0 & 0.00 & - & - & - & - & - \\
\hline Naphthalene $(\mu \mathrm{g} / \mathrm{L})$ & 72 & 0.5 & $<0.5$ & $<0.5$ & 0 & 0.00 & - & - & - & - & - \\
\hline$n$-Butylbenzene $(\mu \mathrm{g} / \mathrm{L})$ & 72 & $<0.5$ & $<0.5$ & $<0.5$ & 0 & 0.00 & - & - & - & - & - \\
\hline$n$-Butyl methyl ketone $(\mu \mathrm{g} / \mathrm{L})$ & 72 & $<1.0$ & $<1.0$ & $<1.0$ & 0 & 0.00 & - & - & - & - & - \\
\hline$n$-Propylbenzene $(\mu \mathrm{g} / \mathrm{L})$ & 72 & $<0.5$ & $<0.5$ & $<0.5$ & 0 & 0.00 & - & - & - & - & - \\
\hline$o$-Xylene $(\mu \mathrm{g} / \mathrm{L})$ & 72 & $<0.5$ & $<0.5$ & $<0.5$ & 0 & 0.00 & 0 & 0 & & 10,000 & - \\
\hline$p$-Isopropyltoluene (4-Isopropyltoluene) $(\mu \mathrm{g} / \mathrm{L})$ & 72 & $<0.5$ & $<0.5$ & $<0.5$ & 0 & 0.00 & - & - & - & - & - \\
\hline $\sec$-Butylbenzene $(\mu \mathrm{g} / \mathrm{L})$ & 72 & $<0.5$ & $<0.5$ & $<0.5$ & 0 & 0.00 & - & - & - & - & - \\
\hline Styrene $(\mu \mathrm{g} / \mathrm{L})$ & 72 & $<0.5$ & $<0.5$ & $<0.5$ & 0 & 0.00 & 0 & 0 & & 100 & - \\
\hline tert-Butylbenzene $(\mu \mathrm{g} / \mathrm{L})$ & 72 & $<0.5$ & $<0.5$ & $<0.5$ & 0 & 0.00 & - & - & - & - & - \\
\hline Tetrachloroethene (Perchloroethylene, PCE) ( $\mu \mathrm{g} / \mathrm{L})$ & 72 & $<0.5$ & $<0.5$ & $<0.5$ & 0 & 0.00 & 0 & 0 & & 5 & - \\
\hline Tetrachloromethane $(\mu \mathrm{g} / \mathrm{L})$ & 72 & $<0.5$ & $<0.5$ & $<0.5$ & 0 & 0.00 & - & - & - & - & - \\
\hline Toluene $(\mu \mathrm{g} / \mathrm{L})$ & 72 & $<0.5$ & $<0.5$ & $<0.5$ & 0 & 0.00 & 0 & 0 & & 1,000 & - \\
\hline trans-1,2-Dichloroethene $(\mu \mathrm{g} / \mathrm{L})$ & 72 & $<0.5$ & $<0.5$ & $<0.5$ & 0 & 0.00 & 0 & 0 & & 100 & - \\
\hline trans-1,3-Dichloropropene $(\mu \mathrm{g} / \mathrm{L})$ & 72 & $<0.5$ & $<0.5$ & $<0.5$ & 0 & 0.00 & - & - & - & - & - \\
\hline Tribromomethane $(\mu \mathrm{g} / \mathrm{L})$ & 72 & $<0.5$ & $<0.5$ & 0.81 & 1 & 1.39 & 0 & 0 & & 60 & - \\
\hline Trichloroethene (TCE, Trichloroethylene) $(\mu \mathrm{g} / \mathrm{L})$ & 72 & $<0.5$ & $<0.5$ & $<0.5$ & 0 & 0.00 & 0 & 0 & & 5 & - \\
\hline Trichloromethane $(\mu \mathrm{g} / \mathrm{L})$ & 72 & $<0.5$ & $<0.5$ & $<0.5$ & 0 & 0.00 & 0 & 0 & & 80 & - \\
\hline Trihalomethanes (THMs), summation $(\mu \mathrm{g} / \mathrm{L})$ & 72 & $<2.0$ & $<2.0$ & 3.43 & 1 & 1.39 & - & - & - & - & - \\
\hline Vinyl Acetate $(\mu \mathrm{g} / \mathrm{L})$ & 72 & $<0.5$ & $<0.5$ & $<0.5$ & 0 & 0.00 & - & - & - & - & - \\
\hline Vinyl Chloride (Chloroethene) ( $\mu \mathrm{g} / \mathrm{L})$ & 72 & $<0.5$ & $<0.5$ & $<0.5$ & 0 & 0.00 & 0 & 0 & & 2 & - \\
\hline Xylene $(\mu \mathrm{g} / \mathrm{L})$ & 72 & $<1.5$ & $<1.5$ & $<1.5$ & 0 & 0.00 & - & - & - & - & - \\
\hline
\end{tabular}

\section{EXPLANATION}




\section{Appendix 3.}

Principal component analysis (PCA) provides insight on hydrochemical processes affecting groundwater chemistry in the study area by indicating intercorrelations among chemical constituents and environmental variables. Six principal components (PCs) explain nearly 78.3 percent of the variance in the groundwater dataset and consist of 26 routinely detected constituent loadings (table 3.1). As explained by Thyne and others (2004), the loading value is comparable to the correlation coefficient between a constituent and the PC, whereas the communality and eigenvalues indicate the degree to which the PCA explains the total variability of the elements considered in the analysis. Associations of additional chemical and physical variables excluded from the model are indicated by the Spearman-rank coefficient of correlation of these variables with the PC scores (table 3.2); significant correlations are listed below the six PCs included in table 3.1, which are identified as principal component 1 (PC1) through principal component 6 (PC6).

PC1 $(\mathrm{pH})$, which explains 36.1 percent of the variance in the data, has positive loadings by boron, lithium, sodium, alkalinity, $\mathrm{pH}$ (field), fluoride, specific conductance (SC) (laboratory), ammonia, and methane, with negative loading by radon-222, nitrate, calcium, and uranium (table 3.2). Scores on PC1 are positively correlated with total dissolved solids (TDS; table 3.2). The negative associations of PC1 with calcium and radon 222 and positive associations with $\mathrm{pH}$, alkalinity, lithium, and sodium are consistent with the progressive reaction of groundwater with minerals along flow paths, where the older water further from recharge areas becomes more mineralized, alkaline, and "softened." Samples classified as anoxic generally had higher, positive scores on PC1 compared to those classified as mixed or oxic, plus PC1 scores were generally greatest for samples from the Lock Haven formation and least for Huntley Mountain, as shown by Appendix figures 4.3 and 4.4. High positive scores on PC1 generally may be attributed to the progressive weathering of calcite and dolomite (carbonate minerals) combined with cation-exchange processes. The naturally occurring cation-exchange reactions liberate sodium and other alkali earth cations, such as lithium, while removing calcium and magnesium (hardness), much like a water-softening treatment system (Hem, 1985; Poth, 1962). The removal of calcium and magnesium from solution leads to undersaturation of the groundwater with respect to calcite and dolomite, thus promoting additional dissolution of the carbonate minerals and progressive increases in $\mathrm{pH}$ and alkalinity along the flow path. The resultant sodium-bicarbonate waters tend to have alkaline $\mathrm{pH}(>8)$ values and high positive scores on PC1 (fig. 3.2). Scores on PC1 also increased with SC class interval (fig. 4.2).

PC2 (Redox), which explains 13.5 percent of the variance in the data, has positive loadings by manganese, iron, ammonia, and methane, and negative loadings by dissolved oxygen, nitrate, and radon-222 (table 3.2). Scores on PC2 are negatively correlated with selenium, copper, and redox potential (Eh). High scores on PC2 are interpreted to indicate isolation from the atmosphere, the depletion of oxygen, and the development of reducing conditions that may be attributed to the microbial decomposition of organic matter in the aquifer or introduced to the groundwater. Samples classified as having anoxic or mixed redox characteristics generally had positive scores on PC2, whereas samples classified as oxic generally had negative scores (fig. 4.3). The negative correlations of copper and selenium with PC2 indicate decreased mobility of these constituents where concentrations of dissolved iron and manganese are elevated, possibly because of adsorption by hydrous ferric oxide (HFO) minerals, including goethite $(\mathrm{FeOOH})$ or ferrihydrite $\left(\mathrm{Fe}(\mathrm{OH})_{3}\right)$, that could be present along with the dissolved iron.

PC3 (Hardness), which explains 8.7 percent of the variance in the data, has positive loadings by sulfate, magnesium, calcium, SC (laboratory), silica, and alkalinity. Scores on PC3 are positively correlated with hardness, nickel, and TDS. High scores on PC3 can be attributed to the dissolution of calcite, dolomite, gypsum, and, possibly, pyrite or other sulfide minerals, without the cation-exchange softening effects (alkaline $\mathrm{pH}$ ) indicated by high scores on PC1. Median PC3 scores decreased in the order of Lock Haven, Catskill, and Huntley Mountain Formations, as shown by Appendix figure 4.4.

PC4 (Chloride-Bromide), which explains 7.2 percent of the variance in the data, has positive loadings by chloride, bromide, SC (laboratory), and sodium and is positively correlated with TDS (table 3.2). The positive association between chloride, bromide, and sodium could indicate the influence of Appalachian Basin brine or other sources of salinity, such as sewage, fertilizer, and (or) road-deicing salt, on the groundwater of associated samples. Scores on PC4 increased progressively with SC class interval (fig. 4.2) and generally were greater for samples from the Catskill Formation than those from the Lock Haven or Huntley Mountain bedrock formations (fig. 4.4).

PC5 (Strontium-Barium), which explains 6.7 percent of the variance in the data, has positive loadings by strontium, potassium, barium, silica, and lithium (table 3.2) and was positively correlated with gross beta activity. The positive associations of the cations and silica could indicate a common origin or geochemical control, such as silicate or carbonate minerals. For example, potassium, barium, and strontium commonly substitute for calcium in carbonate (aragonite) and for one another in sulfate (barite-celestine) minerals, which could be possible sources or sinks of the cations (Hanor, 1968; Hanshaw and Back, 1979). Additionally, these cations may be retained by clay minerals involved in cation exchange (Appelo and Postma, 2005) and, thus, may be released as the exchange sites become depleted in sodium and enriched in calcium and magnesium.

PC6 (Molybdenum-Arsenic), which explains 6.0 percent of the variance in the data, has positive loadings by molybdenum, arsenic, uranium, and radon-222. The positive 
associations of these trace elements and lack of correlation with other constituents, indicates that variations in the traceelement concentrations are independent of variations in the major ions, $\mathrm{SC}, \mathrm{pH}$, or redox.

\section{References Cited}

Appelo, C.A.J., and Postma, D., 2005, Geochemistry, groundwater and pollution: Leiden, The Netherlands, A.A. Balkema Publishers, 649 p.

Hanor, J.S., 1968, Frequency distribution of compositions in the barite-celestite series: American Mineralogist, v. 53, p. $1215-1222$.

Hanshaw, B.B., and Back, W., 1979, Major geochemical processes in the evolution of carbonate-Aquifer systems: Contemporary Hydrogeology-The George Burke Maxey Memorial Volume, v. 43, no. 1, p. 287-312.

Hem, J.D., 1985, Study and interpretation of the chemical characteristics of natural water: U.S. Geological Survey Water-Supply Paper 2254, 263 p., [Also available at https:// doi.org/10.3133/wsp2254.]

Poth, C.W., 1962, The occurrence of brine in western Pennsylvania: Pennsylvania Geological Survey 4th ser., Mineral Resources Report 47, 53 p.

SAS Institute, Inc., 2012, SAS 9.4 for Windows: Cary, N.C., USA, SAS Institute, Inc.

Thyne, G., Guler, C., and Poeter, E., 2004, Sequential analysis of hydrochemical data for watershed characterization: Ground Water, v. 42, no. 5, p. 711-723. 
Table 3.1. Principal components analysis model of major factors controlling the chemistry of groundwater and statistically significant Spearman rank correlations of other variables with those factors determined from chemical properties of and dissolved concentrations of constituents in groundwater samples from 72 wells, Bradford County, Pennsylvania, 2016.

[PC1-PC6, principal components 1 through 6 with major factor indicated in parentheses; Varimax rotation pattern for rank-transformed data (SAS, 2012); minimum eigenvalue $>1$; loading values for constituents included in model and Spearman correlation coefficients for constituents excluded from model multiplied by 100 and rounded; *, indicates highly significant loading or correlation $(\mathrm{p}<0.001)$; + indicates significant loading or correlation $(\mathrm{p}<0.01)$; -, indicates insignificant correlation or not applicable]

\begin{tabular}{|c|c|c|c|c|c|c|c|}
\hline Principal component & $\begin{array}{l}\text { PC1 } \\
\text { (pH) }\end{array}$ & $\begin{array}{c}\text { PC2 } \\
\text { (Redox) }\end{array}$ & $\begin{array}{c}\text { PC3 } \\
\text { (Hardness) }\end{array}$ & $\begin{array}{c}\text { PC4 } \\
\text { (Chloride- } \\
\text { Bromide) }\end{array}$ & $\begin{array}{c}\text { PC5 } \\
\text { (Strontium- } \\
\text { Barium) }\end{array}$ & $\begin{array}{c}\text { PC6 } \\
\text { (Molybdenum- } \\
\text { Arsenic) }\end{array}$ & Communality \\
\hline \multicolumn{8}{|c|}{ Constituent loadings } \\
\hline Boron (B) & $87 *$ & $26+$ & -7 & 9 & $26+$ & 1 & 0.912 \\
\hline Lithium (Li) & $84 *$ & 9 & -13 & 16 & $36 *$ & 3 & 0.891 \\
\hline Sodium $(\mathrm{Na})$ & $79 *$ & $25+$ & -4 & $39 *$ & 10 & 2 & 0.855 \\
\hline pH, Field (pHF) & $69 *$ & $30+$ & $-32+$ & -13 & 10 & 20 & 0.735 \\
\hline Fluoride (F) & $68 *$ & 22 & $-35+$ & 11 & 3 & -10 & 0.660 \\
\hline Specific conductance, lab (SCL) & $56 *$ & 21 & $50 *$ & $53 *$ & 0 & -11 & 0.907 \\
\hline Radon 222 (Rn222) & $-68 *$ & $-37 *$ & -11 & 4 & 3 & $37 *$ & 0.756 \\
\hline Methane (Methane) & $41 *$ & $67 *$ & -16 & 26 & 24 & -9 & 0.781 \\
\hline Nitrate $\left(\mathrm{NO}_{3} \mathrm{~N}\right)$ & $-46^{*}$ & $-66 *$ & 2 & 8 & -16 & 5 & 0.681 \\
\hline Dissolved oxygen (DOX) & -22 & $-74 *$ & 13 & 1 & -16 & -18 & 0.679 \\
\hline Sulfate $\left(\mathrm{SO}_{4}\right)$ & 7 & -9 & $84 *$ & -2 & -22 & 7 & 0.779 \\
\hline Magnesium (Mg) & -18 & 9 & $80 *$ & 1 & 28 & 2 & 0.767 \\
\hline Calcium (Ca) & $-42 *$ & 5 & $73 *$ & 20 & 22 & 21 & 0.853 \\
\hline Chloride $(\mathrm{Cl})$ & 0 & 14 & 3 & $89 *$ & 10 & -6 & 0.833 \\
\hline Bromide (Br) & $31+$ & 14 & -1 & $81 *$ & 10 & -1 & 0.779 \\
\hline Strontium (Sr) & $29+$ & $32+$ & 1 & 6 & $77 *$ & 10 & 0.793 \\
\hline Uranium (U) & $-53 *$ & $-31+$ & 11 & -2 & -15 & $65 *$ & 0.832 \\
\hline \multicolumn{8}{|c|}{ Eigenvalue } \\
\hline Eigenvalue & 9.40 & 3.52 & 2.27 & 1.88 & 1.74 & 1.56 & - \\
\hline Percent Variance Explained & 36.1 & 13.5 & 8.7 & 7.2 & 6.7 & 6.0 & - \\
\hline Cumulative Percent Variance Explained & 36.1 & 49.7 & 58.4 & 65.7 & 72.3 & 78.3 & - \\
\hline \multicolumn{8}{|c|}{ Significant Spearman correlations $(p<0.001)$} \\
\hline Total dissolved solids (TDS) & 57 & - & 46 & 48 & - & - & - \\
\hline Phosphate $\left(\mathrm{PO}_{4} \mathrm{P}\right)$ & 38 & - & - & - & - & - & - \\
\hline Ethane (Ethane) & 37 & 43 & -33 & - & - & - & - \\
\hline Selenium (Se) & - & -48 & - & 36 & - & - & - \\
\hline Copper $(\mathrm{Cu})$ & -44 & -57 & - & - & - & - & - \\
\hline
\end{tabular}


Table 3.1. Principal components analysis model of major factors controlling the chemistry of groundwater and statistically significant Spearman rank correlations of other variables with those factors determined from chemical properties of and dissolved concentrations of constituents in groundwater samples from 72 wells, Bradford County, Pennsylvania, 2016. - Continued

\begin{tabular}{|c|c|c|c|c|c|c|c|}
\hline Principal component & $\begin{array}{l}\mathrm{PC1} \\
(\mathrm{pH})\end{array}$ & $\begin{array}{c}\text { PC2 } \\
\text { (Redox) }\end{array}$ & $\begin{array}{c}\text { PC3 } \\
\text { (Hardness) }\end{array}$ & $\begin{array}{c}\text { PC4 } \\
\text { (Chloride- } \\
\text { Bromide) }\end{array}$ & $\begin{array}{c}\text { PC5 } \\
\text { (Strontium- } \\
\text { Barium) }\end{array}$ & $\begin{array}{c}\text { PC6 } \\
\text { (Molybdenum- } \\
\text { Arsenic) }\end{array}$ & Communality \\
\hline \multicolumn{8}{|c|}{ Significant Spearman correlations $(\mathrm{p}<0.001)$-Continued } \\
\hline Redox potential (EHmv) & - & -64 & - & - & - & - & - \\
\hline Hardness (HRDcalc) & - & - & 79 & - & - & - & - \\
\hline Nickel (Ni) & - & - & 48 & - & - & - & - \\
\hline $\begin{array}{l}\text { Gross beta radioactivity, 30-day count, water, filtered, } \\
\text { Cesium-137 curve, picocuries per liter (BetaCs30) }\end{array}$ & - & - & - & - & 51 & - & - \\
\hline $\begin{array}{l}\text { Gross beta radioactivity, } 72 \text {-hour count, water, filtered, } \\
\text { Cesium-137 curve, picocuries per liter (BetaCs72) }\end{array}$ & - & 30 & - & - & 47 & - & - \\
\hline $\begin{array}{l}\text { Gross alpha radioactivity, } 30 \text {-day count, water, filtered, } \\
\text { Th-230 curve, picocuries per liter (AlphaTh30) }\end{array}$ & - & - & - & - & 38 & - & - \\
\hline $\begin{array}{l}\text { Gross alpha radioactivity, } 72 \text {-hour count, water, filtered, } \\
\text { Th-230 curve, picocuries per liter (AlphaTh72) }\end{array}$ & - & - & - & - & - & 34 & - \\
\hline Cadmium (Cd) & -32 & - & - & - & - & - & - \\
\hline Lead $(\mathrm{Pb})$ & -32 & -31 & - & - & - & - & - \\
\hline Antimony (Sb) & - & -31 & - & - & - & - & \\
\hline Well depth (WELLZ) & 31 & -32 & - & - & - & - & - \\
\hline $\mathrm{pH}, \mathrm{Lab}(\mathrm{pHL})$ & 69 & - & -33 & - & - & - & - \\
\hline Residue on evaporation 180C (ROE180) & 58 & - & 43 & 48 & - & - & - \\
\hline Specific conductance, field (SCF) & 53 & - & 47 & 53 & - & - & - \\
\hline Aluminum (Al) & - & - & - & - & - & - & - \\
\hline Beryllium (Be) & - & - & - & - & - & - & - \\
\hline Cobalt (Co) & - & - & - & - & - & - & - \\
\hline Land-surface altitude (LSALT) & - & - & - & - & - & - & - \\
\hline Propane (Propane) & - & - & - & - & - & - & - \\
\hline Total coliform (Tcoli) & - & - & - & - & - & - & - \\
\hline Temperature (TEMPC) & - & - & - & - & - & - & - \\
\hline Depth to water (WATRZ) & - & - & - & - & - & - & - \\
\hline Well bottom altitude (WELLZALT) & - & - & - & - & - & - & - \\
\hline Zinc (Zn) & - & - & - & - & - & - & - \\
\hline
\end{tabular}


[r-values multiplied by 100 and rounded; only values significant at $\mathrm{p}<0.01$ shown; PC, principle component; -, not significant; abbreviations are defined in table 3.1]

\begin{tabular}{|c|c|c|c|c|c|c|c|c|c|c|c|c|c|c|c|c|c|c|c|c|c|c|c|c|c|c|c|c|c|c|c|c|c|c|c|c|c|c|c|c|c|c|c|c|c|c|c|c|c|c|c|c|}
\hline Param & $\begin{array}{c}\text { Parameter } \\
\text { abbreviation }\end{array}$ & $\bar{\Xi}$ & & & & & 道 & & & & & 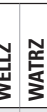 & & & & & & ex & & & & & & & & & & & & & $\bar{\varepsilon}$ & & ® $\overline{0}$ & 8 & & & & & & $\bar{\Sigma}$ s & $\Sigma$ & के & & & 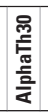 & & & & & & 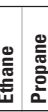 & \\
\hline $\begin{array}{l}\text { Principal } \\
\text { component } 1\end{array}$ & $\mathrm{PC} 1$ & 100 & - & . & - - & - & - & - & - & - & -3 & $31-$ & 69 & 69 & 55 & 535 & 5857 & & -35 & - & $\begin{array}{ll}40 & 79\end{array}$ & $\begin{array}{ll}79 & 72\end{array}$ & 32 & - & 66 - & - . & 55 & & 38 & & - & - - & - -32 & & -44 & & 285 & - & & - 39 & & - & & 89 - & - & & & & $6-52$ & 433 & 37 & \\
\hline $\begin{array}{l}\text { Principal } \\
\text { component } 2\end{array}$ & 2 & -16 & 00 - & & - & - & - & -75 & & & $-{ }^{-3}$ & 32 - & 30 & - & - & - & $-\cdot-$ &.- & - & - & $-3 c$ & $30 \quad 32$ & - & $\cdot$ & $\cdot-$ &.- & 70 & -66 & - & - & - & $-\cdot$ & $\cdot-$ & $\cdot-$ & -5776 & $76-31$ & & 87 & $\cdot-$ & - 33 & & -31 & -34 & $30-48$ & 8 - & - & - 30 & & $8-34$ & 714 & 43 & \\
\hline $\begin{array}{l}\text { Principal } \\
\text { component } 3\end{array}$ & 3 & - & & 00 - & -- & - - & - & - & - & & - & - - - & -33 & -33 & 46 & 474 & 4346 & 679 & 72 & 80 & $\cdot \cdot$ & - 36 & - & $\cdot$ & -4 & $\begin{array}{ll}4585 \\
\end{array}$ & $5-$ & - & - & $\cdot$ & $\cdot-$ & -44 & - & - & $\cdot-$ & $-\cdot$ & - & - & -48 & $48-$ & $\cdot$ & - & -- & $-\cdot$ & - & - & - & - & - & . & & \\
\hline $\begin{array}{l}\text { Principal } \\
\text { component } 4\end{array}$ & 54 & & 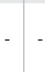 & -10 & & $\cdot$ & - & - & - & $\cdot$ & $\cdot-$ & $-\quad-$ & - & - & 51 & \begin{tabular}{ll|l}
53 & 4
\end{tabular} & $48 \quad 48$ & $8-$ & - & $\cdot$ & -37 & \begin{tabular}{l|l}
37 & -
\end{tabular} & 83 & 89 & $\cdot-$ & - & $\cdot$ & - & - & - & - & 31 - & $-\cdot$ & $\cdot$ & $\cdot-$ & $-\cdot$ & - & - & $-\cdot$ & $-\cdot$ & $\cdot$ & - & $\cdot-$ & -36 & - & - & - & - & - & - & & \\
\hline $\begin{array}{l}\text { Principal } \\
\text { component } 5\end{array}$ & 5 & - & - - & & -100 & & - & - & - & - & - - & - - - & - & - & - & - & - - & - - & - & - & 71 - & - - & - & - & -55 & 59. & $\cdot-$ & - & - & - & - & 58 - & $\cdot-$ & - & - - & - - & 42 & - & $-\cdot$ & - 77 & $7 \cdot$ & - & -33 & 33 - & 38 & -5 & $\begin{array}{ll}51 & 47\end{array}$ & & - & 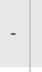 & & \\
\hline $\begin{array}{l}\text { Principal } \\
\text { component } 6\end{array}$ & C6 & - & - - & & & 10 & & - & - & $\cdot$ & - - & - - - & - & - & - & - & - - & . & - & - & - . & -.- & - & $\cdot$ & - - & - - &. & - & - & - & - & - - - & \begin{tabular}{l|l}
- & - \\
&
\end{tabular} & $\cdot$ & & - - & - & -9 & 93 - & - - & - & -7 & $\begin{array}{ll}77- \\
-\end{array}$ & -. & - & 34 & & 36 & 65 & - & - & \\
\hline Temperature & TEMPC & - & - - & &.- & & 100 & & - & $-36-$ & -35 & - & - & - & $\cdot$ & - & - - & - & - & - & - & $\cdot-$ & - & $\cdot$ &.- & - &. & - & - & 32 & - & $-\cdot$ & - & $\cdot$ & $\cdot-$ & $\cdot-$ & - & - & - - & - - & 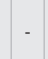 & - & $-\quad-$ & - - & - & $\cdot$ & - & - & - & - & - & \\
\hline Dissolved oxygen & DOX & -1 & -75 - & - . & - - - &.- & - & 100 & & - &. & & & $8-43$ & - & - & - - &. & - & - & & $-38-38$ & - & - & -36. &.- & & 55 & - & & - & $-31-$ & & -5 & & 42 - & & -56 & & - -40 & & - & & -4536 & - & & & & & -5 & & \\
\hline Redox potential & EHmv & 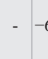 & 64 & $\cdot$ & - - & $-\cdot$ & - & 35 & 100 & & - & - & - & - & - & - & - - & - & - & - & $-\cdot$ & $-\cdot$ & - & - & $\cdot-$ & $-\cdot$ & & 42 & - & & - & $\cdot-$ & - & 4 & $40-6$ & 65 - & & -57 & & - & & - & - & 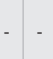 & - & - & & 32 & 39 & -52 & & \\
\hline $\begin{array}{l}\text { Land-sur } \\
\text { altitude }\end{array}$ & LSALT & - & - & - . & - - & - & -36 & & & 100 & 923 & $30-$ & - & - & - & - & - - &.- & - & - & - & $-\cdot-$ & - & . & . & - - & - & & - & - & - & $-36-$ & - & - & - & - & - & - & & - & 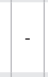 & - & - & - & - & - & & - & - & - & & \\
\hline $\begin{array}{l}\text { Well botto } \\
\text { altitude }\end{array}$ & LLZZALT & - & & & - - & - & -35 & & - & 921 & $100-$ & - & - & - & - & - & - - & - - & - & - & - & - $-\cdot$ & - & - & - & - - - & - - & & - & - & - & $-34-$ & - & - & - & - & - & - & & & 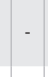 & - & - & 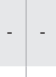 & $\cdot-$ & - & & - & - & - & & \\
\hline Well depth & WELLZ & 31 & $-32-$ & .. & - - - &.- & - & - & . & 30 & -10 & 0063 & - & - & - & - & - - &. & - & - & & - - & - & - &. & - . &. & & - & - & - & - . & & - & & .. & & -38 & & - & ( & - & - & . & - & - & & & - & - & & \\
\hline Depth to water & WATRZ & - & . . & - . & - . & - & - & - & - & $\cdot$ & -6 & 63100 & & - & - & - & - - & - & $\cdot$ & - & $-\cdot$ & $\cdot-$ & - & - & $-\cdot$ &. & - & - & - & & - & $-58-$ &.- & - & & -51 - & - & - & & & & - & - - & & - & - & - & & & 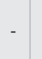 & - & \\
\hline pH, Field & p & $\begin{array}{ll}693 \\
3\end{array}$ & $30-3$ & $33-$ &. &.- & - & -48 & & - &. & - - - & & 088 & - & - & & & 7-41 & - & & \begin{tabular}{l|l}
54 & 45 \\
\end{tabular} & . &. & $\begin{array}{ll}64 & -\end{array}$ & - . & 50 & -50 & & - & - & 32 - &. & & & $31-35$ & 566 & . & & 3936 & & - & & 66 & - & & & & & $\begin{array}{ll}44 & \end{array}$ & 41 & \\
\hline $\mathrm{pH}, \mathrm{Lab}$ & $\mathrm{p}$ & 69 & & $33-$ & - . & - & - & -43 & & - &. & - . & 88 & 100 & & - & - . & -4 & $1-42$ & - & -60 & \begin{tabular}{l|l}
60 & 44
\end{tabular} & - & . & 66 - & - . & 50 & -48 & & 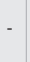 & 31 & 38 & & -33 & -50 . & & 766 & - & & 3536 & -32 & 2 & -7 & 71 & - & - & & & $3-37$ & 47 & 44 & \\
\hline $\begin{array}{l}\text { Specific conduc- } \\
\text { tance, lab }\end{array}$ & $\mathrm{s}$ & 55 & & $\begin{array}{ll}46 & 51\end{array}$ & $51-$ & - & - & - & - & - &.- & - - - & - & - & 100 & & & & - & 31 & \begin{tabular}{l|l}
32 & 70
\end{tabular} & $\begin{array}{ll}70 & 83\end{array}$ & 58 & 50 & \begin{tabular}{|l|l}
41 & -
\end{tabular} & - 37 & 756 & & 41 & & - & - . & - . & . & & 40 & 48 & 44 & & - - & & - & -5 & 54 & - & - & & & $5-37$ & 42 & & \\
\hline $\begin{array}{l}\text { Specific } \\
\text { tance, fi }\end{array}$ & $\mathrm{S}$ & 53 & & $47 \quad 53$ & $53-$ & - & - & - & - & - &.- & - - - & - & - & 98 & 1009 & 9598 & $\begin{array}{ll}833 \\
\end{array}$ & - & 32 & & 6781 & 58 & 52 & 41. & -40 & 053 & & 40 & & - & - - & & - & -4241 & 41 & 46 & 43 & & - - - & & - & -5 & 52 & - & - & & & 3-34 & 38 & & \\
\hline $\begin{array}{l}\text { Residue on } \\
\text { evaporation 180C }\end{array}$ & E180 & 58 & & $43 \quad 48$ & 48 & - & - & - & - & . &. & - - - & - & - & 96 & 951 & & & - & - & 3572 & 7281 & 56 & 46 & \begin{tabular}{|l|l}
46 & - \\
\end{tabular} & - 37 & 753 & & 42 & - & - &.- & - . & . & & 38 & 49 & 43 & & - . & - & - & .5 & 56 - & - & - & & & $4-37$ & 43 & & \\
\hline $\begin{array}{l}\text { Total dissolved } \\
\text { solids }\end{array}$ & TDS & 57 & & $\begin{array}{ll}46 & 48\end{array}$ & 18 & - & - & - & - & . &. & - . & - &. & 100 & 989 & $97 \quad 10$ & & - & - & 3271 & 7184 & 56 & 48 & 43. & - 38 & 858 & & 42 & - & - & - - & & - & -4443 & 43 & 49 & 45 & & - - & - & - & & 55 - & - & - & - & & -39 & 45 & & \\
\hline Hardness & HRDcalc & - & & 79 & - & - & - & - & - & - & - . & - . & & $7-41$ & 31 & 33 & & & $\begin{array}{lll}0 & 98\end{array}$ & 88 & - $\cdot$ & $-\cdot$ & - &. & -334 & \begin{tabular}{l|l}
4153 \\
1
\end{tabular} & 3 & $\cdot$ & - & - & - & $-\cdot$ & - - & - & $-\cdot$ & - & - & - & & $46-$ & $\cdot$ & . & & -31 - & - & - & & & 34 & - & - & \\
\hline Calcium & c & -35 & & 72 & - - - & - & - & - & - & - & $-\cdot$ & & -41 & $1-42$ & & - & - - - & -98 & & 77 & - . & - - - & - & & -3638 & 3851 & 1 & - & - & - & - & - - & \begin{tabular}{l|l}
- & -
\end{tabular} & - & $\cdot-$ & . & -33 & - & & 46 - & $\cdot$ & - & -3 & & - & - & & & 39 & & - & \\
\hline Magnesium & $\mathrm{Mg}$ & & & 30 & - & 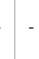 & & - & - & - & - & - & - & - & 31 & 32 & & 88 & 77 & 100 & & - 31 & & - & & 1449 & 9 & & & - & & - & - & 30 & $\cdot-$ & - & 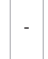 & 37 & & 36 - & 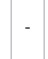 & - &. &. & - & - & & & - & - & - & \\
\hline
\end{tabular}


Table 3.2. Spearman rank correlation coefficient ( $r$ ) matrix for ground-water chemical data Bradford County, Pennsylvania, 2016. - Continued

\begin{tabular}{|c|c|c|c|c|c|c|c|c|c|c|c|c|c|c|c|c|c|c|c|c|c|c|c|c|c|c|c|c|c|c|c|c|c|c|c|c|c|c|c|c|c|c|c|c|c|c|c|c|c|c|c|c|}
\hline Parameter name & $\begin{array}{c}\text { Parameter } \\
\text { abbreviation }\end{array}$ & $\bar{\Xi}$ & & & & & & & & & 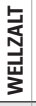 & & & & & & & & 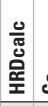 & & & & & & & & & & & & $\bar{\alpha}$ & & 중 & 8 & ¿: & & ב & & & $\bar{z}$ is & $\pi$ & के & & & & 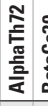 & & & & 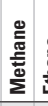 & & \\
\hline Potassium & K & 40 & & - & -7 & 71 . & - & - & - - & - & - & - & - & - . & -3 & 32. & 35 & 32 & - & -3 & $\begin{array}{ll}1100 \\
100\end{array}$ & 38 & 32. & - . & - & 31 & -3 & 5. & . & . & -3 & 31. & - & . & . . & .. & 52 & - & - . & - 58 & & - & - 4 & $13=$ & - & -4 & \begin{tabular}{l|l}
44 & 58
\end{tabular} & & - & 34 & & \\
\hline Sodium & $\mathrm{Na}$ & 79 & 30 & & \begin{tabular}{l|l}
37. &
\end{tabular} & - . &. & -3 & $88-$ & - & - & - & -5 & \begin{tabular}{l|l}
54 & 6
\end{tabular} & \begin{tabular}{l|l}
60 & 7
\end{tabular} & $\begin{array}{ll}70 & 6\end{array}$ & 772 & 71 & - &. & -38 & 100 & \begin{tabular}{l|l}
69 & 6
\end{tabular} & $\begin{array}{lll}60 & 36\end{array}$ & 56 & - & - 6 & $8-5$ & 156 & - &. & - . & - & - & -474 & & 478 & 34 & - & - 41 & - & - & -8 & $\begin{array}{ll}34 & -\end{array}$ & - &.- & - 32 & & & \begin{tabular}{l|l}
65 & 4
\end{tabular} & 46 & \\
\hline Alkalinity & ALK & 72 & 32 & 36 & . & - . & . & -3 & 88 & - & - & - & -4 & \begin{tabular}{l|l}
45 & 4
\end{tabular} & $\begin{array}{ll}448 \\
8\end{array}$ & $\begin{array}{ll}83 & 81\end{array}$ & 181 & 84 & - & - 31 & 132 & 691 & 1004 & 41 - & 49 & - & -6 & $3-5$ & 444 & - & - & - . & -31 & 1. & -534 & & 1061 & 45 & - & - 34 & & - & - 6 & 99 - & - & - & - . & & -31 & 463 & 36 & \\
\hline Bromide & $\mathrm{Br}$ & 32 & - & $\begin{array}{ll}- & 8\end{array}$ & 83. & - . &. & . & - & - & - & - & 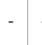 &.- & - 5 & \begin{tabular}{|l|l}
58 & 58
\end{tabular} & \begin{tabular}{l|l}
856 \\
\end{tabular} & 56 & - & - . &.- & 60 & \begin{tabular}{l|l}
41 & 10
\end{tabular} & 0070 & - & - & - 4 & 1. & 32 &. &. & - . & - &. & -3632 & & 45 & - & - & - . & - & - & - 4 & 13 . & - &.- & . . . & - & - & \begin{tabular}{|l|l}
46 & 3 \\
\end{tabular} & 32 & \\
\hline Chloride & $\mathrm{Cl}$ & - & - & -8 & 89. & - . & . & & . & - & - & - & - &. & -5 & $\begin{array}{ll}50 & 52\end{array}$ & 246 & 48 & - & - . & - & 36 & & $\begin{array}{lll}70 & 100\end{array}$ & & - & 3 & 3 & - & - & -3 & 38 - & - & $\cdot$ & - &.- & - & - & - & - . & - & - &.- & - . & - &. & - 31 & & - & 33 & & \\
\hline Fluoride & F & 66 & - & - & - . & - . &. & -3 & 36. & - & - & - & -6 & \begin{tabular}{l|l}
64 & 6
\end{tabular} & \begin{tabular}{l|l}
66 & 4
\end{tabular} & \begin{tabular}{l|l}
41 & 4
\end{tabular} & $\begin{array}{l}146 \\
14\end{array}$ & 43 & $-33-$ & -36 . & - . & 56 & $\begin{array}{ll}49 & .\end{array}$ & - . & 100 & & - 5 & \begin{tabular}{ll|l}
8 & -38 \\
\end{tabular} & 842 & - &.- & - . & -33 & 3. & -5736 & & 1060 & - & - & - . & - & - & -6 & $54-$ & - & - & - . & -63 & & 444 & 46 & \\
\hline Silica & $\mathrm{SiO}_{2}$ & $\cdot$ & - & 45 & - 5 & 99. & .. & & - . & - & - & - & - & - . & - . & - . & .. & - & $\begin{array}{ll}41 & 3\end{array}$ & $\begin{array}{ll}38 & 44\end{array}$ & $\begin{array}{ll}431 \\
4\end{array}$ & - & . . & - . & . & 100 & 31. & - . & - & - &. & - . & - & - & - . & - . & . & - & - & - 42 & - & - & - . & - . & - & - & - . & - & - & - & & \\
\hline Sulfate & $\mathrm{SO}_{4}$ & - & - & 85. &.- & - - - &. & &. & - & - & - & - &.- & -3 & 374 & $\begin{array}{ll}0 & 37\end{array}$ & 38 & 535 & \begin{tabular}{l|l}
51 & 49
\end{tabular} & 9 - & - &. & - . & - & 31 & 100. &. & - & - & -- & -52. & - & - &. &. & - & - & -4 & 43 - & - & - &. &. & -32 & - & - . & - &. & - & -39. & \\
\hline Ammonia & $\mathrm{NH}_{3} \mathrm{~N}$ & 55 & 70 & - & - . & - . & . & & $99-51$ & 1 - & - & - & -5 & \begin{tabular}{l|l}
50 & 5
\end{tabular} & \begin{tabular}{|l|l}
50 & 5
\end{tabular} & \begin{tabular}{l|l}
56 & 53
\end{tabular} & 353 & 58 & - & - . & -35 & 68 & \begin{tabular}{l|l}
63 & 4
\end{tabular} & 4133 & 358 & - & -10 & $00-72$ & 238 & - & -3 & 31. & - & - & -6771 & & 1361 & 62 & . . & - 56 & & -36 & -7 & 72 - & - & -3 & \begin{tabular}{l|l}
30 & 49
\end{tabular} & & & 835 & 54 & \\
\hline Nitrate & $\mathrm{NO}_{3} \mathrm{~N}$ & -48 & -66 &.- &. & - - - &. & & $\begin{array}{ll}5 & 42\end{array}$ & - & - & $\cdot$ & -- & $-50-4$ & $-48-3$ & $36-3$ & $32-33$ & -37 & - & - . & - & -51 & -54. & - . & -38 & 8. & --7 & 72100 & 0 - & - &.- & - . & & - & $64-5$ & 5239 & $9-48$ & -55 & - & - -42 & 2. & 38 & --6 & 6345 & - &.- & - . & 46 & 47 & & -41. & \\
\hline Phosphate & $\mathrm{PO}_{4} \mathrm{P}$ & 38 & - & $\cdot$ & - . & - . & - & & - . & - & - & - & - & - & - 4 & $414 c$ & 042 & 42 & - & - . &.- & 56 & 443 & 32 - & 42 & - & -3 & 8 - & 100 & - & - & - . & - & - & -36 & 36. &. & - & - . & - . & - & - & -3 & $38-$ & - &.- & - . & -33 & - & 344 & 43 & \\
\hline Total coliform & Tcoli & - & &.- & - . & & - 32 & & . & & - & - & & - & - . & - . & - & - & - & - . & - - & - &. & - . & & - &. & - & - & 100 & - & - . & & - &.- & &. & - &. & - - & - & - & - & $\cdot$. & - &.- &. & - & - & - & - & \\
\hline Aluminum & $\mathrm{Al}$ & - & - &.- & - . &.- & - - & &. & - & - & - & - & -3 & 31. & - . &. & - & - & - . & - . & - &. & - . & - & - &. & - & - & -1 & 100 & - 35 & 5 - & - & - . & - . &. & - & - & - . & - & 31 & - & - . & - &. &. & - & - & - & - & \\
\hline Barium & $\mathrm{Ba}$ & - & - & -443 & & 58. &.- & & $31-$ & -36 & -34 & $\cdot$ & -583 & $32 \quad 3$ & 38. &.- & - & - & - & - . - & - 31 & - &. & - 38 & 8. & - & -523 & 1 - & - & - & -11 & $100-$ & & - &.- &.- & 36 & - & - & - 53 & - & - & - &.- & 40 & \begin{tabular}{l|l}
- & 4
\end{tabular} & \begin{tabular}{l|l}
45 & 4 \\
\end{tabular} & & - & 40 & 48 & \\
\hline Beryllium & $\mathrm{Be}$ & - & - & . & -. & - . & - . & & . & - & - & - & $\cdot$ &.- & - . & - . & - & - & - &.- & - . & - & - . & - . & - & - &. & - . & - & - & 35 & - 10 & 0 - & 32 & . . & - . &. & $\cdot$ & - & - . & - & - & - & - . & - &. &. & - & - & - & - & \\
\hline Cadmium & $\mathrm{Cd}$ & -32 & - & - &.- &.- & - . & &. & - & - & - & - & --3 & -31. & - . & - & - & - &.- &.- & - & -31. & & -33 & 3. & - & - & - & - &.- & - . & 100 & 0. &. & - . & -32 & - & - & - - & 33 & - & - & 33 - &. & - &.- & & - & - & - & \\
\hline Cobalt & Co & - & . & - &.- & - . & - . & - & . & - & . & - & - & --3 & -33. & . . . & . & - & - & -3 & $30-$ & - & - & - & - & - & - & . & - & - &.- & - 32 & & 100 & &.- &. & - & - & 50 & - & - & - & - . & - & 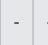 & - & - & - & - & - & \\
\hline Copper & $\mathrm{Cu}$ & -44 & & & & & - & 55 & \begin{tabular}{c|c}
5 & 40
\end{tabular} & & - & - & - & $-57-4$ & $-50-4$ & & $42-41$ & -44 & & & & $\mid-47-$ & & & -57 & 7 & $--r$ & 6764 & 4 & . & - & . & . & & $100-5$ & 5354 & & -55 & & - -40 & & 41 & & 5539 & & & & 256 & 50 & & -39 & \\
\hline Iron & $\mathrm{Fe}$ & - & 76 & &.- & & - . & & $42-65$ & & . & - & -513 & 31 & -4 & $\begin{array}{ll}40 & 41\end{array}$ & 138 & 43 & - & . & - & 46 & $\begin{array}{ll}40 & 3 \\
\end{array}$ & 32 - & 36 & 5 & - & $\begin{array}{lll}1 & -52\end{array}$ & 236 & - & - & . . &. & . & -5310 & & 1032 & 70 & - & - 36 & & . & - & 11 & - & . & 4 & -55 & & 623 & 39 & \\
\hline Lead & $\mathrm{Pb}$ & -32 & & & - & & - . . & & . & - & - & - & -- & $-35-1$ & & - & & - & . & & & $-34-$ & -40. & & -40 & 0 & & 4339 & & . & - & & - & & & & $00-32$ & -38 & & - & - & 46 & & 3835 & & & -3 & 136 & 34 & & -34 & \\
\hline Lithium & $\mathrm{Li}$ & 85 & & - & & 42. & - & -3 & 34 & - & - & - & -6 & \begin{tabular}{l|l}
66 & 6
\end{tabular} & \begin{tabular}{l|l}
66 & 4
\end{tabular} & $\begin{array}{ll}48 \quad 4 c \\
\end{array}$ & 649 & 49 & & -33 & - 52 & 78 & $\begin{array}{ll}61 & 4\end{array}$ & 45 & 60 & - & & $51-48$ & & . & -3 & 36 & -32 & & -4432 & & 32100 & & - & - 56 & & - & - & 0 & - & . & 33 & & -47 & 55 & 46 & \\
\hline Manganese & $\mathrm{Mn}$ & - & 87 & &.- & & - & & $56-57$ & & & -38 & & - & - 4 & $44 \quad 4$. & 343 & 45 & - & & 37. & 34 & 45 & & - & - & & $2-5$ & & - & - & - & - & - & -557 & $70-38$ & 8 & 100 & & - 34 & & - & - & $11-46$ & & - & 4 & -37 & 7 & 61 & 30 & \\
\hline Molybdenum & Mo & - & - & & & - 93 & 3 & & - . & & & - & - & - & - & - & & & - & - & & - & & . & & & & & & - & & & & & -1 & - . & & & 100 & & . & & 63 & - & & 36 & . & & 55 & - & - & \\
\hline
\end{tabular}




\begin{tabular}{|c|c|c|c|c|c|c|c|c|c|c|c|c|c|c|c|c|c|c|c|c|c|c|c|c|c|c|c|c|c|c|c|c|c|c|c|c|c|c|c|c|c|c|c|c|c|c|c|c|}
\hline Parameter name & $\begin{array}{c}\text { Parameter } \\
\text { abbreviation }\end{array}$ & हू & & & & & & & & & & & & & & & & & 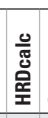 & & & & & & & & $0_{2}^{z}$ & 定 & $\bar{\alpha}$ & Ф & ¿ & & ڤ & & $\Xi \Sigma$ & & $\bar{z}$ is & $\bar{N}$ & & & ळ & 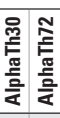 & 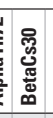 & & & ح. & 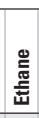 & \\
\hline Nickel & $\mathrm{Ni}$ & - & & 48 & & - & - & - &.- & - - & - & - & - & $-39-$ & -35 & . & - &. & 46 & $\begin{array}{lll}463 \\
\end{array}$ & 6. &. & - &. & - 4 & 43. & . & . - &. & . . & -5 & 50 . &. &. &. & & 100 - & - & 43. & - . & - &. & . & & & . & - & \\
\hline Strontium & Sr & 39 & 33 & - & - & 77 & - & --4 & 40. & $-\cdot-$ & - & - & - & 36 & 36 & - &.- & - . & - &. & 58 & \begin{tabular}{|l|l|}
41 & 34 \\
\end{tabular} & $\cdot$ & - - & 42 & - 56 & -42 &. &. & $53-$ & - & - -40 & 1036 & -5 & $\begin{array}{ll}66 & 34\end{array}$ & - & -100 & 0 - & . . & - 56 & -3 & \begin{tabular}{l|l}
37 & -
\end{tabular} & 61 & \begin{tabular}{|l|l}
58 &.
\end{tabular} & & 3149 &.- & \\
\hline Zinc & $\mathrm{Zn}$ & - & $\cdot$ & - & - & - & - &.- &.- & - . & - & - & - & - & -32 & $\cdot$ & - & . . & . &. &. &. & . & . - &. & . - & . & . - &. & . . & 33 & . - &. &. &. & . & . . & 100 & . . & . . & - &. & . &.- & & - & - & - \\
\hline Antimony & Sb & $\cdot$ & -31 & - & - & - & - & &.- & - . & - & - & - & & - & - & - &. & . & &. &. & . &.- &. & -36 & 638 &. & 31 &. &.- & - 41 & 1. & \begin{tabular}{|l|l}
46 & -
\end{tabular} &.- & - 4 & 43 - & - & 10033 & 3. & 32 &. &. &. & &.- & - & - \\
\hline Arsenic & As & - & - & - & - &. & 77 & -. &.- & - . & - & - & - & - & - & - & - & . . & . &. & $\cdot$ &. & . &. &. &. & . & . - &. & . . &. & . - &. &. &.- & 63 & - . & - & 3310 & 00 - & . &. & - & - & -3 & $3=$ & - & - \\
\hline Boron & B & 89 & 30 & & . & 33 & - & -1 & 45. & - . & & - & - & 66 & $71:$ & 545 & \begin{tabular}{l|l}
52 & 5 \\
\end{tabular} & 5655 & -31 & -35 & 43 & \begin{tabular}{|l|l|}
84 & 69
\end{tabular} & 43 & - 64 & 4. & - 72 & -63 & 38 - & $\cdot$ &. & -33. & & 541 . & -389 & \begin{tabular}{l|l|}
30 & 31
\end{tabular} & & - 56 & 6. & - . & -100 & - &. & . & $35-6$ & $63-$ & 5662 & 48 & - \\
\hline Selenium & Se & - & -48 & - & 36 & - & - & -3 & 36. & .. & - & - & - & - & - & - &. & - . & - &. & - &. & . & . - &. & . . & 45 & . - &. & . - &. & - 39 & . & 35 & -46 & 6. & . . & - & 32. & . . & 100 &. & . & -3 & $\begin{array}{ll}34 . \\
.\end{array}$ & . . & - & - \\
\hline $\begin{array}{l}\text { Gross alpha } \\
\text { radioactivity, 30- } \\
\text { day count, water, } \\
\text { filtered, Th-230 } \\
\text { curve, picocuries } \\
\text { per liter }\end{array}$ & AlphaTh30 & - & - & & - & 38 & - & &.- &.- & & - & - & & - & - & - & - - - & - & & - & $-\cdot-$ & - & $\cdot-$ & - - & -32. & - & $\cdot-$ & - & 40 - & - & - - - & - &.- & - - & & - 37 & 7. & - - &. & -1 & $\begin{array}{ll}10066 \\
6\end{array}$ & 551 & 35 . & &.- & - & - \\
\hline $\begin{array}{l}\text { Gross alpha } \\
\text { radioactivity, } 72- \\
\text { hour count, water, } \\
\text { filtered, Th-230 } \\
\text { curve, picocuries } \\
\text { per liter }\end{array}$ & AlphaTh72 & - & - & - & - & -3 & 34 & - &.- & - . & - & - & - & - & - & - & - & - - & - & -. & $-\cdot-$ & - - - & - & - - & $\cdot$ & - - & - & - - & - & - - & - & - - & - & - - & - - & 36 & - - & - & - - & - - & -6 & & 050 & 51 & & - - - & - & - \\
\hline $\begin{array}{l}\text { Gross beta } \\
\text { radioactivity, 30- } \\
\text { day count, water, } \\
\text { filtered, Cs-137 } \\
\text { curve, picocuries } \\
\text { per liter }\end{array}$ & BetaCs30 & - & - & & - & 51 & - & - &.- & -. & - & - & - & - & - & - & -. & - - - & - & .. & 44 & $-\cdot-$ & - & - - & -. & - 30 & - & - - & - & 45 - & - & - - & - &. & -. & - & - 61 & 1. & - . & -.- & -5 & \begin{tabular}{l|l}
51 & 50
\end{tabular} & & 59 & &.- & - & - \\
\hline $\begin{array}{l}\text { Gross beta } \\
\text { radioactivity, } 72- \\
\text { hour count, water, } \\
\text { filtered, Cs-137 } \\
\text { curve, picocuries } \\
\text { per liter }\end{array}$ & BetaCs72 & - & 30 & & - & 47 & & - & - & -. & - & - & - & - & - & - & - . & - - & - & - & - 58 & $32-$ & -3 & 31 - & - & - 49 & & - - & - & 46 - & - & & 243 & -313 & $35 \quad 40$ & - & - 58 & & - - & - 35 & -3 & \begin{tabular}{l|l}
35 & 51
\end{tabular} & 59 & 100 & & 44 & - & - \\
\hline Radon 222 & Rn222 & -66 & & & - & -3 & 36 &.- & -3 & 32. & - & - & - & -54 & $-53-$ & $-55-$ & $-53-5$ & $-54-58$ & &. &. & $-59-55$ & 5. & --63 & 3. & - -66 & $646-$ & $-33=$ &. & . . & - & - 56 & & $36-5$ & $51-37$ & . & - & - & - . & & 34 & . & . & & & & $1-45$ & \\
\hline Uranium & U & -52 & & & - & & 65 & - & -3 & 39 & - & $\cdot$ & - & & & & & & 34 & 39. & $\cdot$ & $-49-31$ & & --58 & 8. & - -62 & 247 &. & - & . . & . & - 50 & & $34-4$ & 47 - & 55 & --31 & & -33 & $3-56$ & & & - & .7 & & & 6-54 & \\
\hline Methane & Methane & 43 & 71 & & - & - & - & & $59-5$ & 52 & . & - & - & 44 & 474 & \begin{tabular}{l|l}
42 & 3
\end{tabular} & $\begin{array}{ll}38 & 4\end{array}$ & 4345 & . & . & -34 & \begin{tabular}{|l|l|}
65 & 46 \\
\end{tabular} & 46 & $\begin{array}{ll}33 & 44\end{array}$ & $4 \cdot-$. & . 83 & $3-62$ & \begin{tabular}{|c|c}
34 & -
\end{tabular} & - & 40 - & - & & 862 . & -405 & 5561 & - & - 49 & 9 & $\cdot$. & $\begin{array}{l}-62 \\
-62\end{array}$ & . & . & . & 44 & & 56100 & 67 & - \\
\hline Ethane & Ethane & 37 & 43 & & & - & - & --4 & $46-3$ & 36. & - & - & - & 41 & 44 & & $\cdot$ & - - & $\cdot$ & . & - & $\begin{array}{ll}46 & 36\end{array}$ & 32 & - 46 & . & -3954 & $4-41$ & 43. & - & 48 & - & & $939-$ & & 4630 & - & - & - & - & - 48 & - & - & - & -- & & 5467 & & 34 \\
\hline Propane & Propane & - & - & & - & - & & & - & - & & & & & & - & & - & & & \begin{tabular}{ll|}
- & - \\
\end{tabular} & $-\cdot$ & - & - & - & \begin{tabular}{l|l}
- & -
\end{tabular} & - & - & - & - - & - & - - & - &.- & - - & & - & - & & - & - & \begin{tabular}{l|l}
- & -
\end{tabular} & - & $-I_{-1}^{-}$ &.- &.- & 34 & \\
\hline
\end{tabular}




\section{Appendix 4.}

Distributions of continuous variables were compared among different sample classifications using notched boxplots (Velleman and Hoaglin, 1981; Helsel and Hirsch, 2002). Where the median for a group is greater than the common reporting limit, it is displayed as a horizontal line within the box that is defined by the 25 th and 75 th percentiles for that group; otherwise, the median is displayed at the reporting limit. If the notched intervals around the medians for sample subsets do not overlap, the medians are statistically different at the 95-percent confidence interval.

\section{References Cited}

Helsel, D.R., and Hirsch, R.M., 2002, Statistical methods in water resources: U.S. Geological Survey Techniques of Water-Resources Investigations, book 4, chap. A3, 510 p., accessed February 2019 at https://doi.org/10.3133/ twri04A3.

McMahon, P.B., and Chapelle, F.H., 2008, Redox processes and water quality of selected principal aquifer systems: Groundwater, v. 46, no. 2, p. 259-271, accessed October 16, 2017, at http://onlinelibrary.wiley.com/doi/10.1111/j.17456584.2007.00385.x/abstract.

Pennsylvania Bureau of Topographic and Geologic Survey, 2001, Bedrock geology of Pennsylvania: Pennsylvania Bureau of Topographic and Geologic Survey, Department of Conservation and Natural Resources, digital data, accessed October 2017 at http://www.denr.state.pa.us/topogeo/map1/ bedmap.aspx.

Velleman, P.F., and Hoaglin, D., 1981, Applications, basics, and computing of exploratory data analysis: Boston, Mass., Duxbury Press, $354 \mathrm{p}$. 

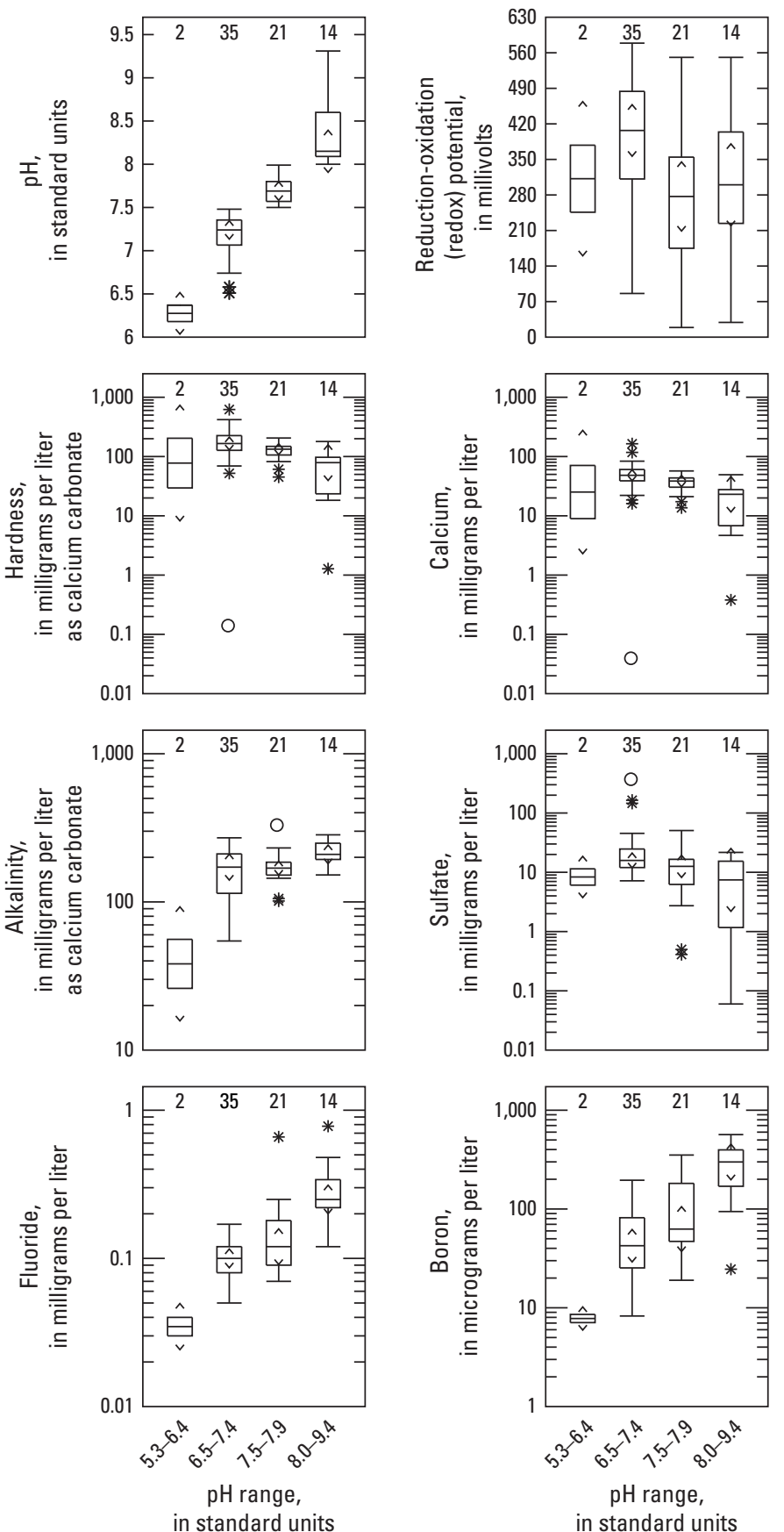
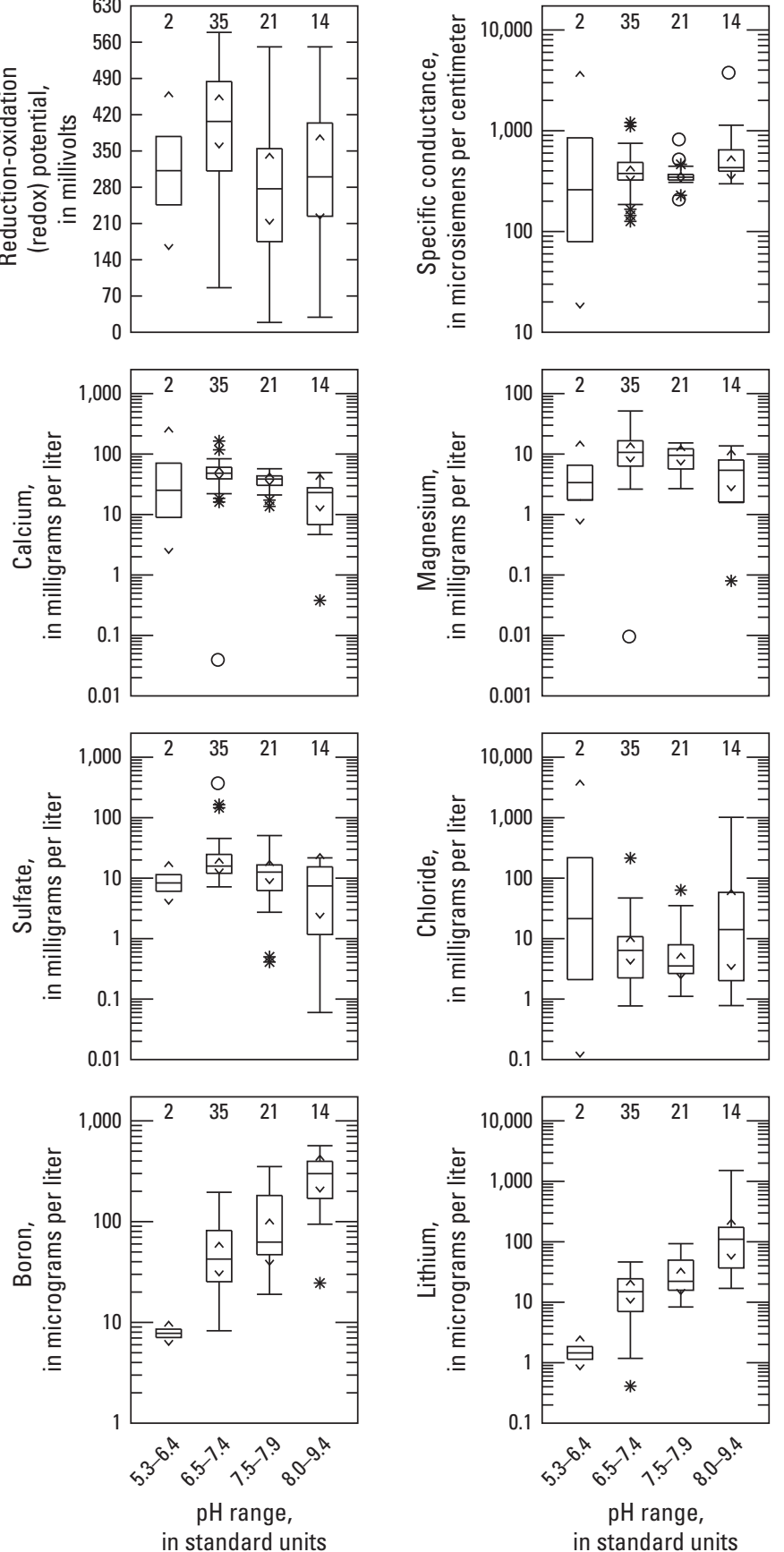

$\mathrm{pH}$ range, in standard units
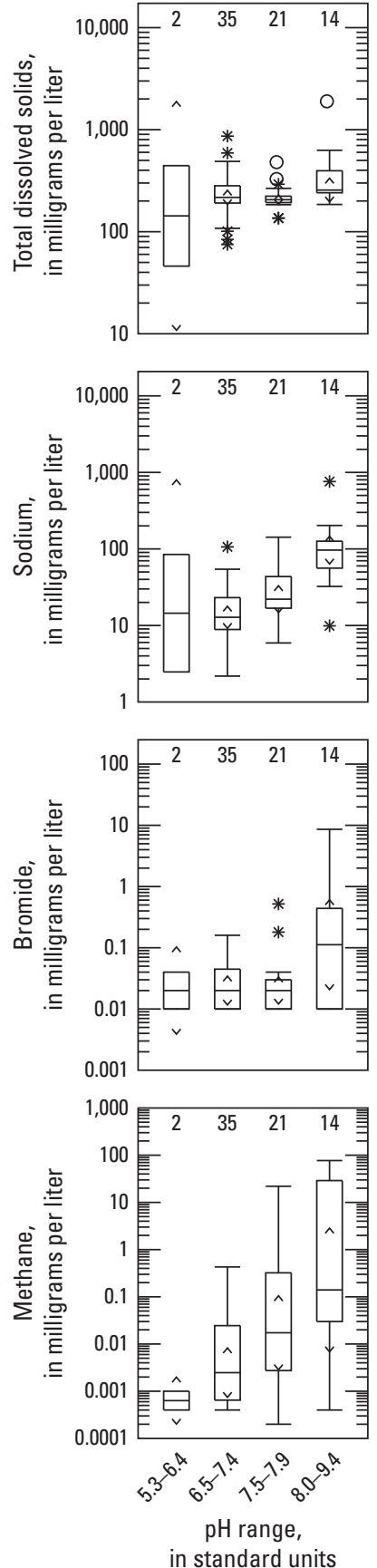

EXPLANATION

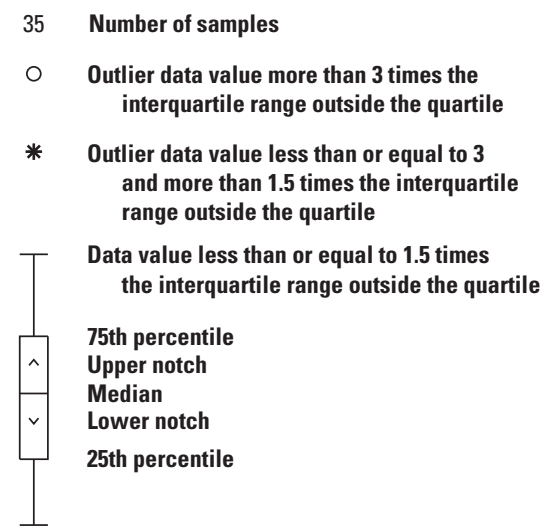

Figure 4.1. Composition of 72 groundwater samples from Bradford County, Pennsylvania, 2016, classified by $\mathrm{pH}$ class interval. The $\mathrm{pH}$ range is indicated on the $\mathrm{x}$-axis $(5.3<\mathrm{pH}<6.4$, $\mathrm{n}=2 ; 6.5<\mathrm{pH}<7.4, \mathrm{n}=35 ; 7.5<\mathrm{pH}<7.9, \mathrm{n}=21 ; 8.0<\mathrm{pH}<9.4$, $\mathrm{n}=14)$. $(<$, less than; $\mathrm{n}$, number of samples; see table 3.1 for descriptions of principal components) 

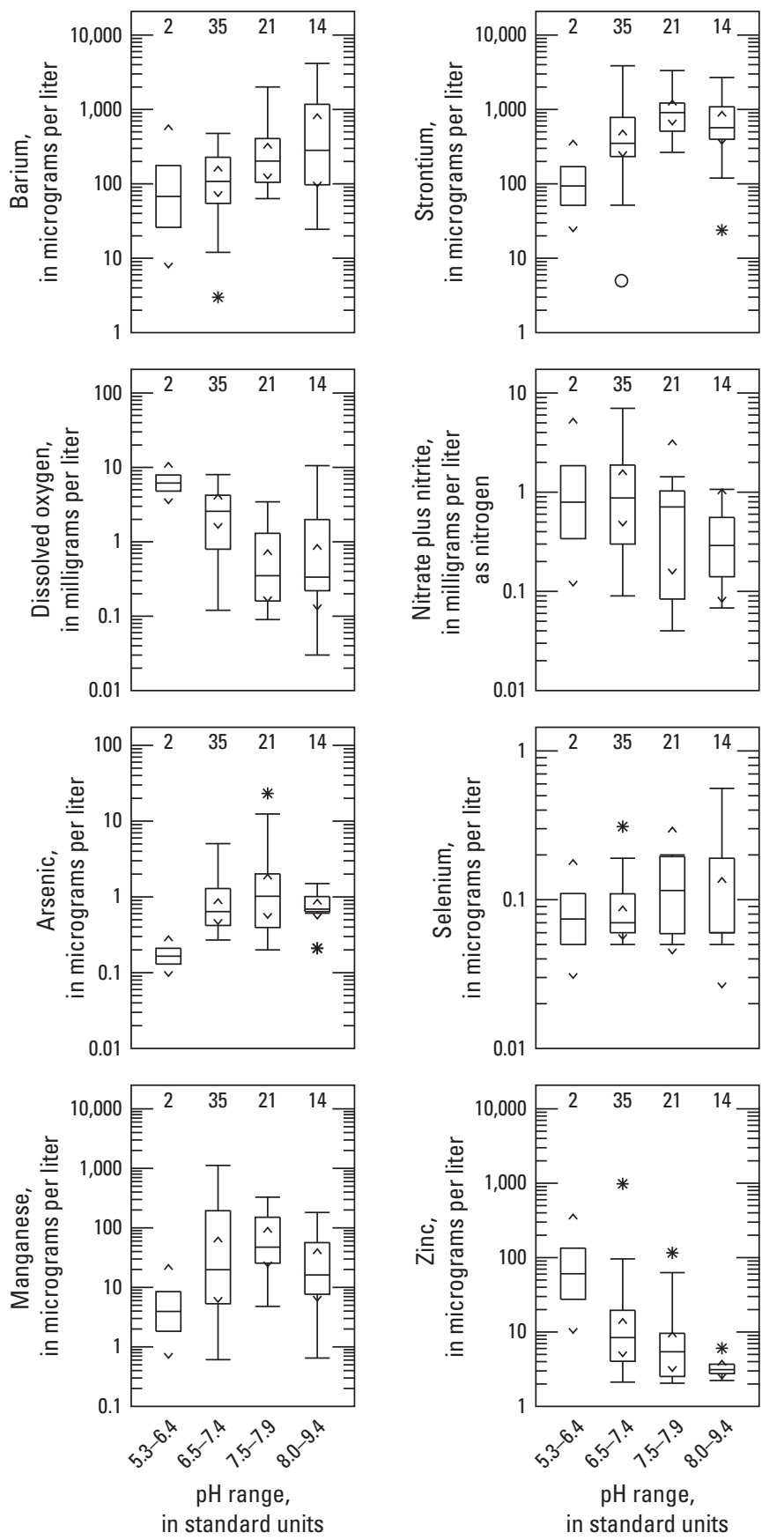

Figure 4.1. Composition of 72 groundwater samples from Bradford County, Pennsylvania, 2016, classified by pH class interval. The $\mathrm{pH}$ range is indicated on the $\mathrm{x}$-axis $(5.3<\mathrm{pH}<6.4$, $\mathrm{n}=2 ; 6.5<\mathrm{pH}<7.4, \mathrm{n}=35 ; 7.5<\mathrm{pH}<7.9, \mathrm{n}=21 ; 8.0<\mathrm{pH}<9.4$, $n=14) .(<$, less than; $n$, number of samples; see table 3.1 for descriptions of principal components)—Continued
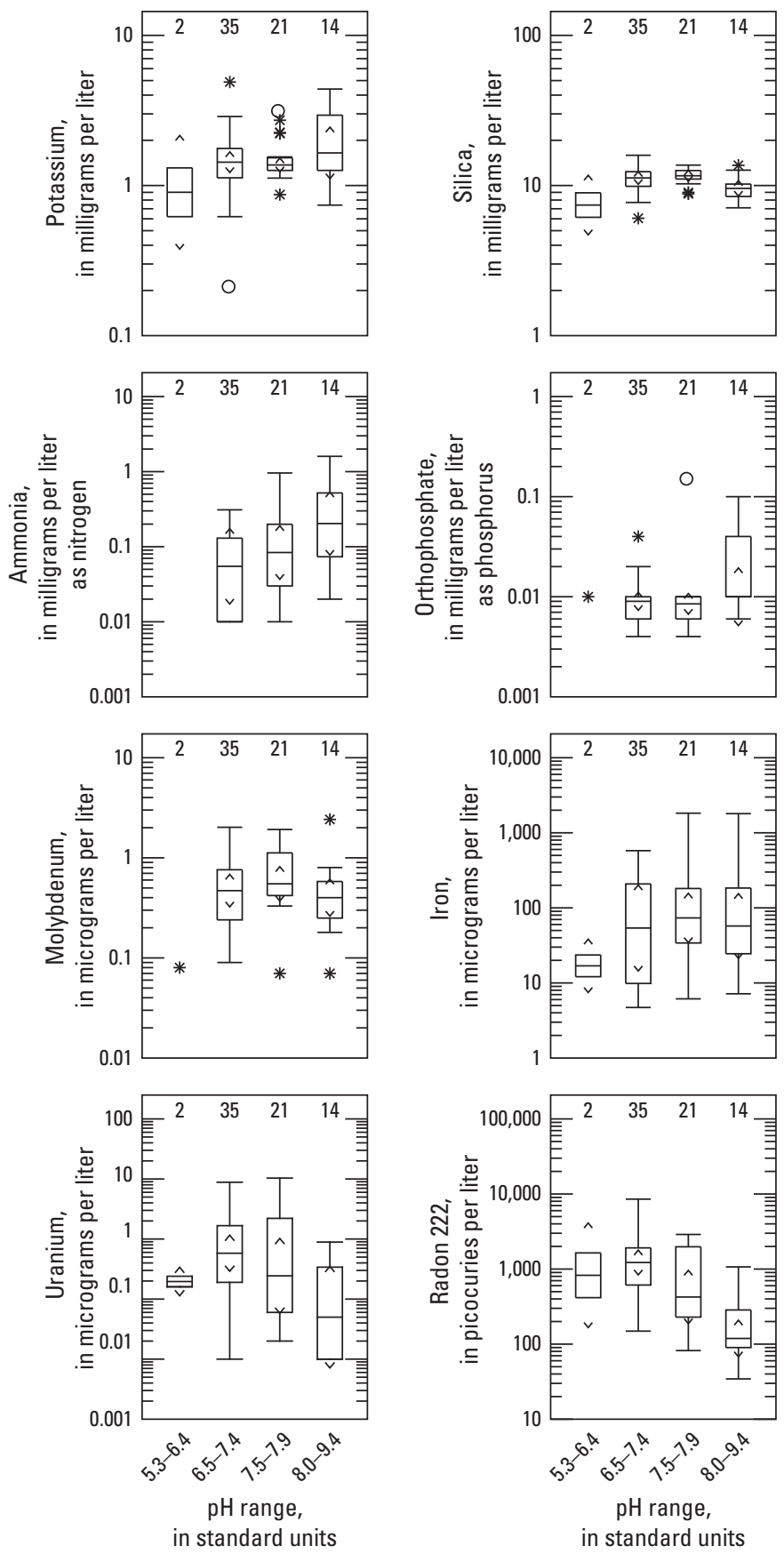

EXPLANATION

$35 \quad$ Number of samples

O Outlier data value more than $\mathbf{3}$ times the interquartile range outside the quartile

* Outlier data value less than or equal to 3 and more than 1.5 times the interquartile range outside the quartile

Data value less than or equal to $\mathbf{1 . 5}$ times the interquartile range outside the quartile

75th percentile

Upper notch

Median

Lower notch

25th percentile 

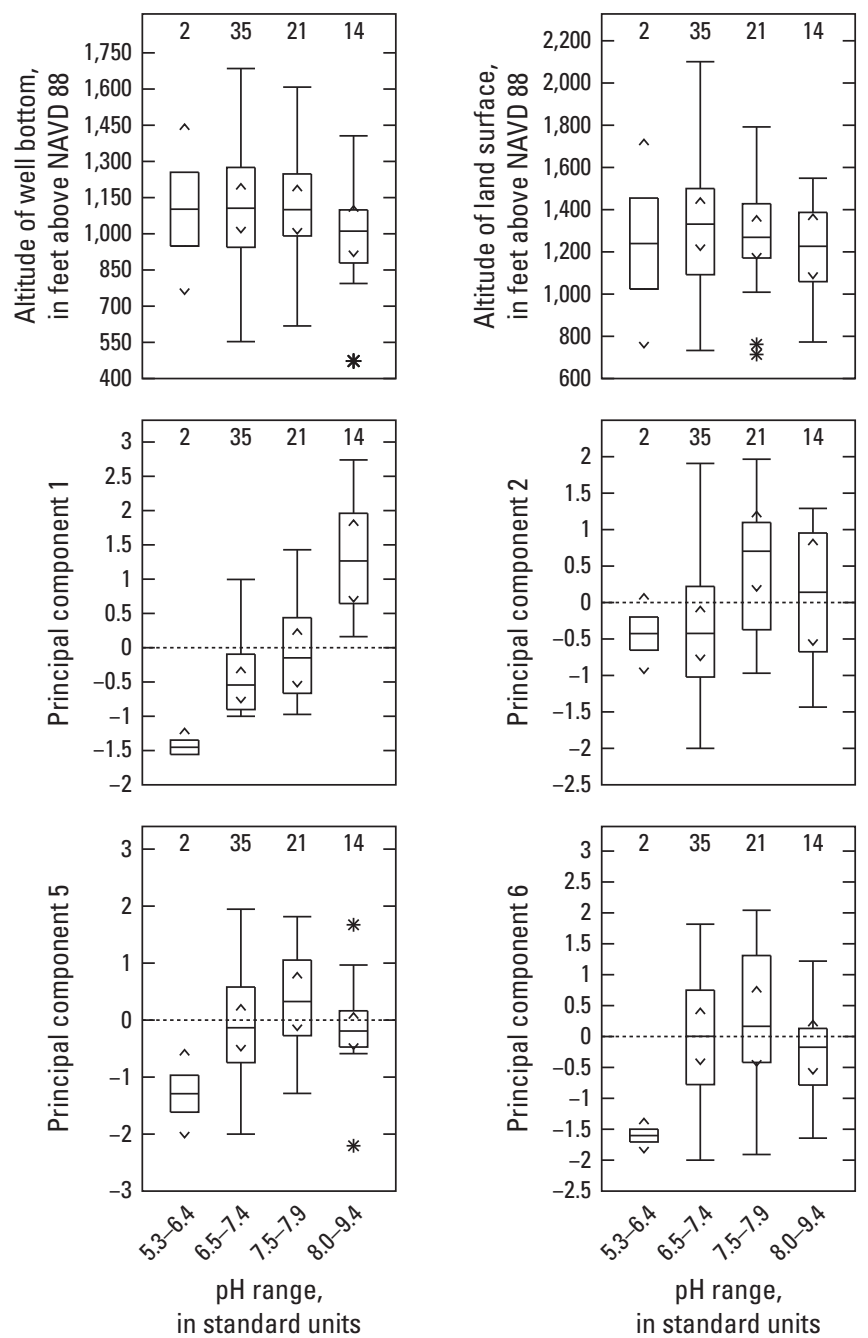

Figure 4.1. Composition of 72 groundwater samples from Bradford County, Pennsylvania, 2016, classified by $\mathrm{pH}$ class interval. The $\mathrm{pH}$ range is indicated on the $\mathrm{x}$-axis $(5.3<\mathrm{pH}<6.4$, $\mathrm{n}=2 ; 6.5<\mathrm{pH}<7.4, \mathrm{n}=35 ; 7.5<\mathrm{pH}<7.9, \mathrm{n}=21 ; 8.0<\mathrm{pH}<9.4$, $\mathrm{n}=14)$. $(<$, less than; $\mathrm{n}$, number of samples; see table 3.1 for descriptions of principal components)—Continued

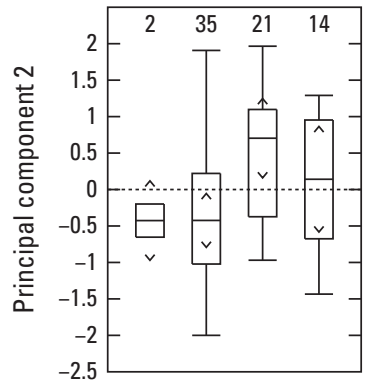

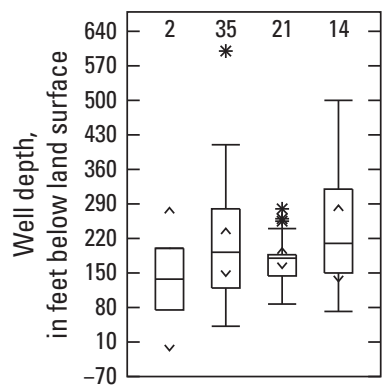
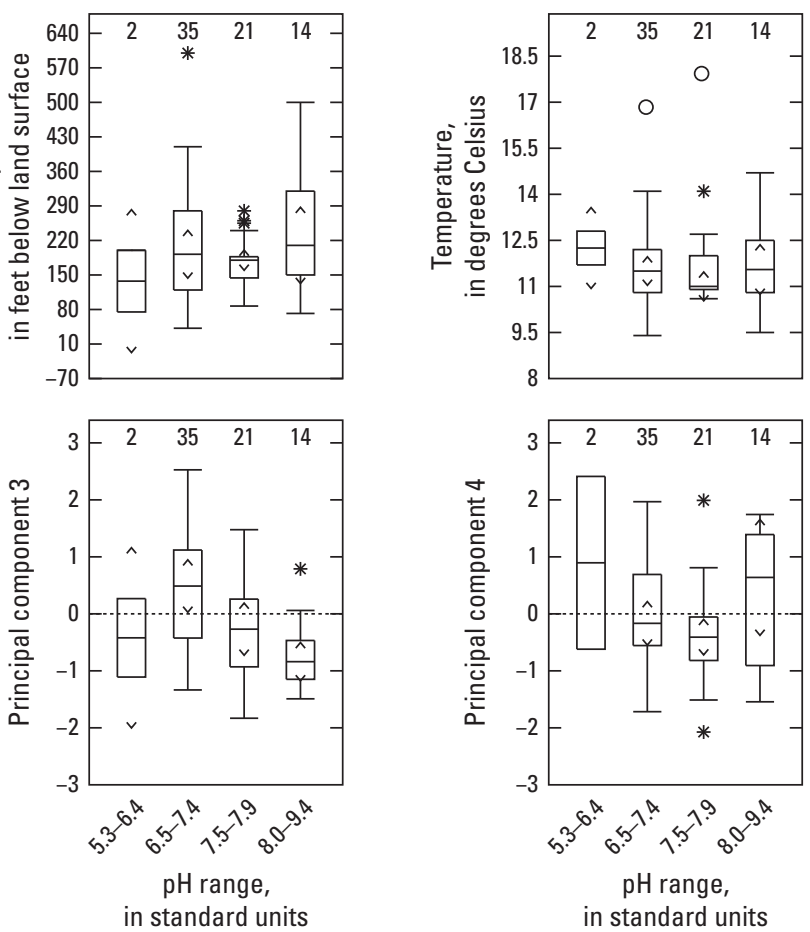

EXPLANATION

Outlier data value more than 3 times the interquartile range outside the quartile

* Outlier data value less than or equal to 3 and more than 1.5 times the interquartile range outside the quartile

Data value less than or equal to 1.5 times the interquartile range outside the quartile

75th percentile

Upper notch

Median

Lower notch

25th percentile 

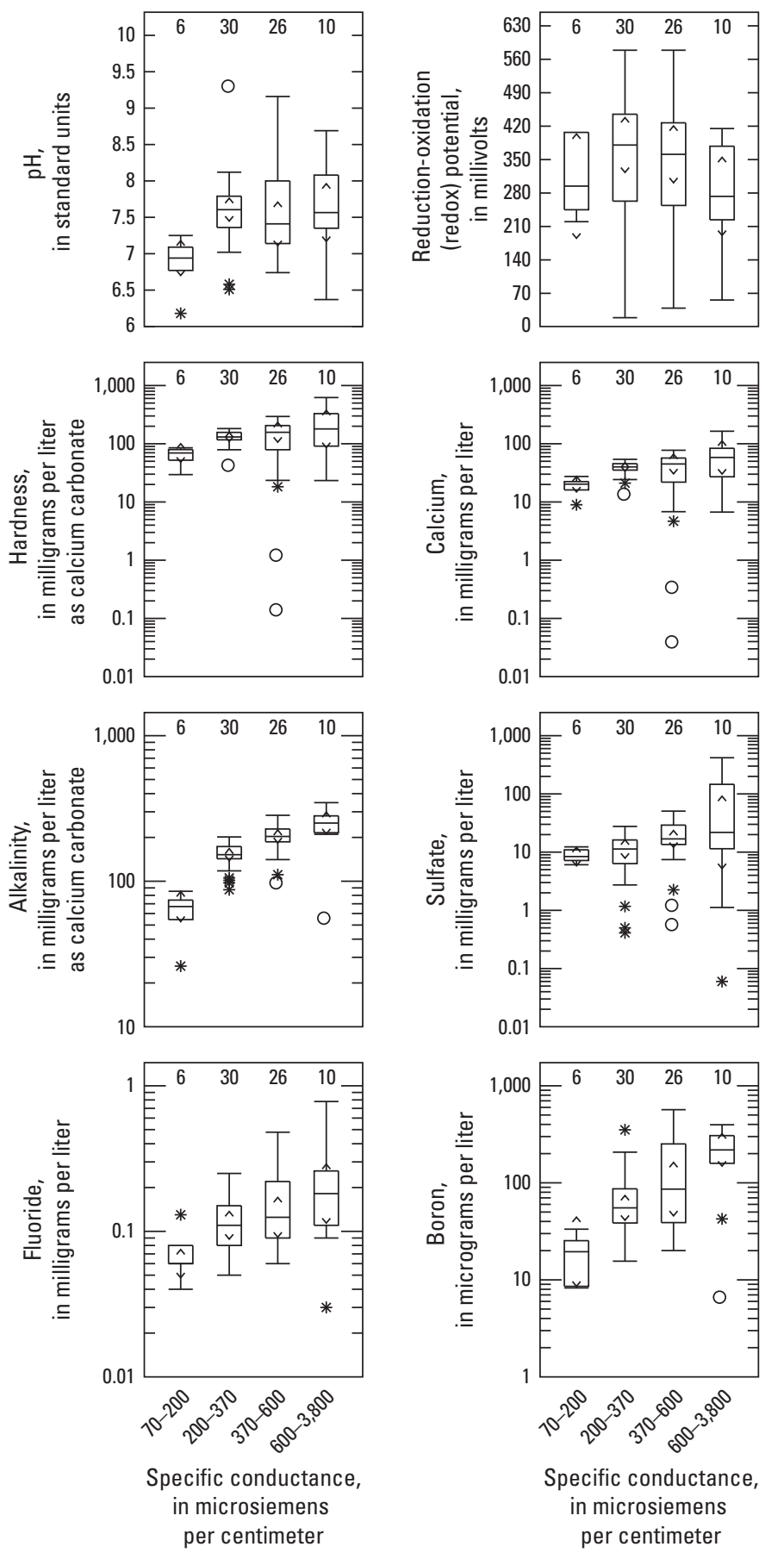

Figure 4.2. Composition of 72 groundwater samples from Bradford County, Pennsylvania, 2016, classified by specific conductance (SC) class interval. The SC range is indicated on the $\mathrm{x}$-axis $(70<\mathrm{SC}<200 \mu \mathrm{S} / \mathrm{cm}, \mathrm{n}=6 ; 200<\mathrm{SC}<370 \mu \mathrm{S} / \mathrm{cm}, \mathrm{n}=30$; $370<\mathrm{SC}<600 \mu \mathrm{S} / \mathrm{cm}, \mathrm{n}=26 ; 600<\mathrm{SC}<3,800 \mu \mathrm{S} / \mathrm{cm}, \mathrm{n}=10)$. $(<$, less than; $\mu \mathrm{S} / \mathrm{cm}$, microsiemens per centimeter; $\mathrm{n}$, number of samples; see table 3.1 for descriptions of principal components)
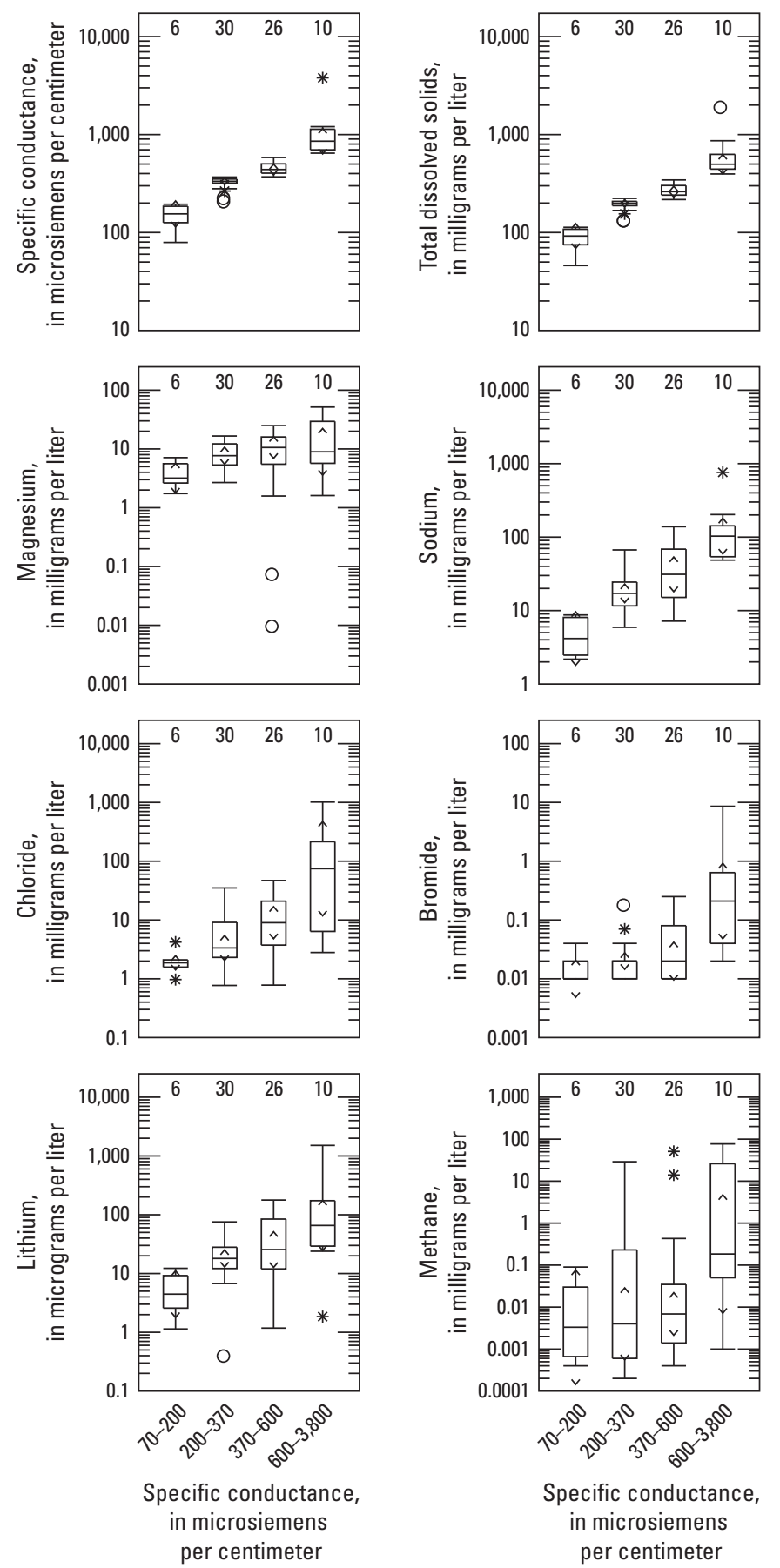

EXPLANATION

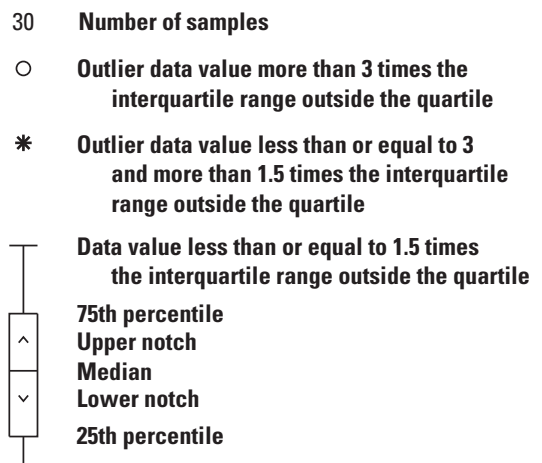



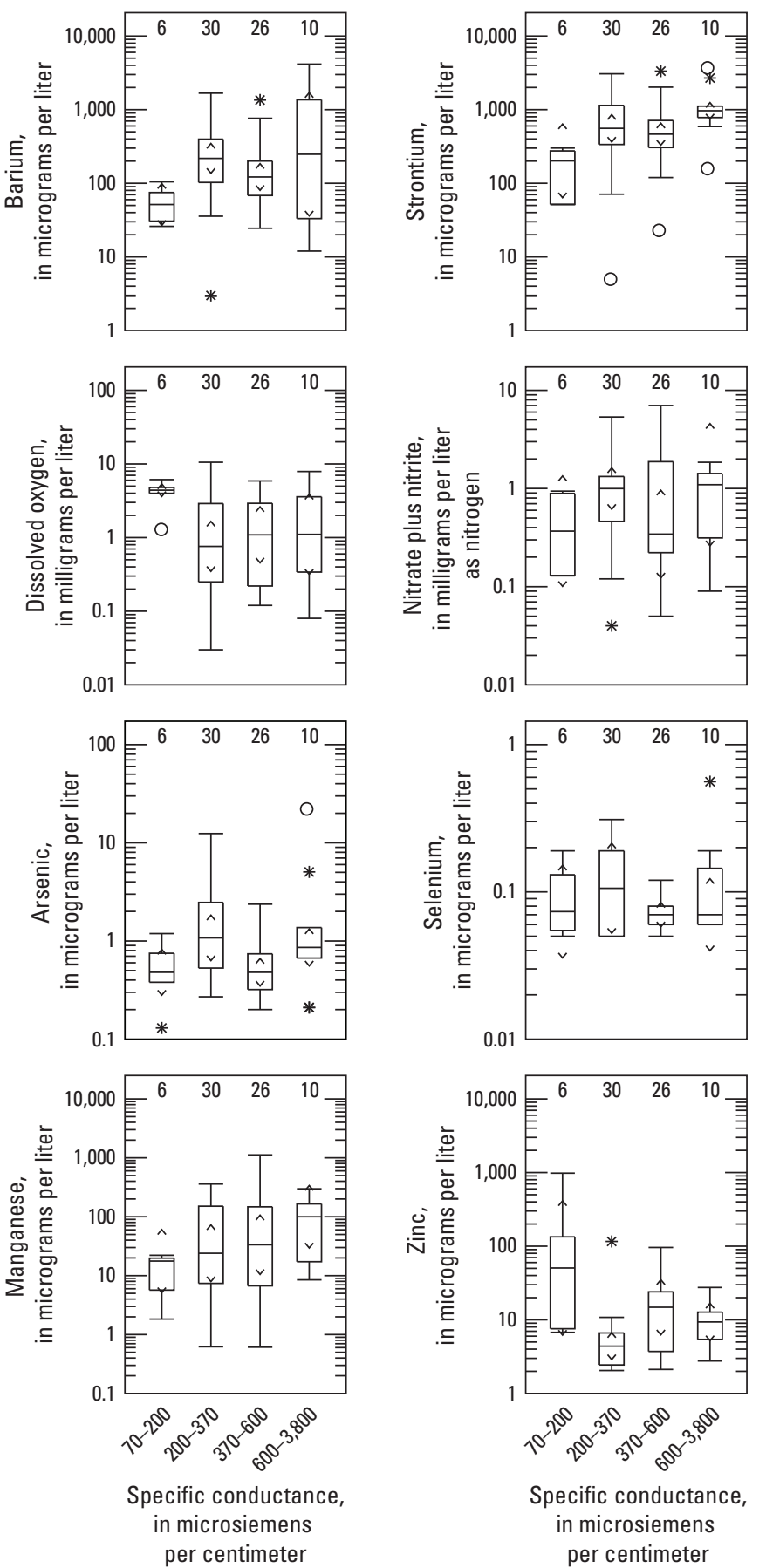

Figure 4.2. Composition of 72 groundwater samples from Bradford County, Pennsylvania, 2016, classified by specific conductance (SC) class interval. The SC range is indicated on the $\mathrm{x}$-axis $(70<\mathrm{SC}<200 \mu \mathrm{S} / \mathrm{cm}, \mathrm{n}=6 ; 200<\mathrm{SC}<370 \mu \mathrm{S} / \mathrm{cm}, \mathrm{n}=30$; $370<\mathrm{SC}<600 \mu \mathrm{S} / \mathrm{cm}, \mathrm{n}=26 ; 600<\mathrm{SC}<3,800 \mu \mathrm{S} / \mathrm{cm}, \mathrm{n}=10$ ). $(<$, less than; $\mu S / c m$, microsiemens per centimeter; $n$, number of samples; see table 3.1 for descriptions of principal components) Continued
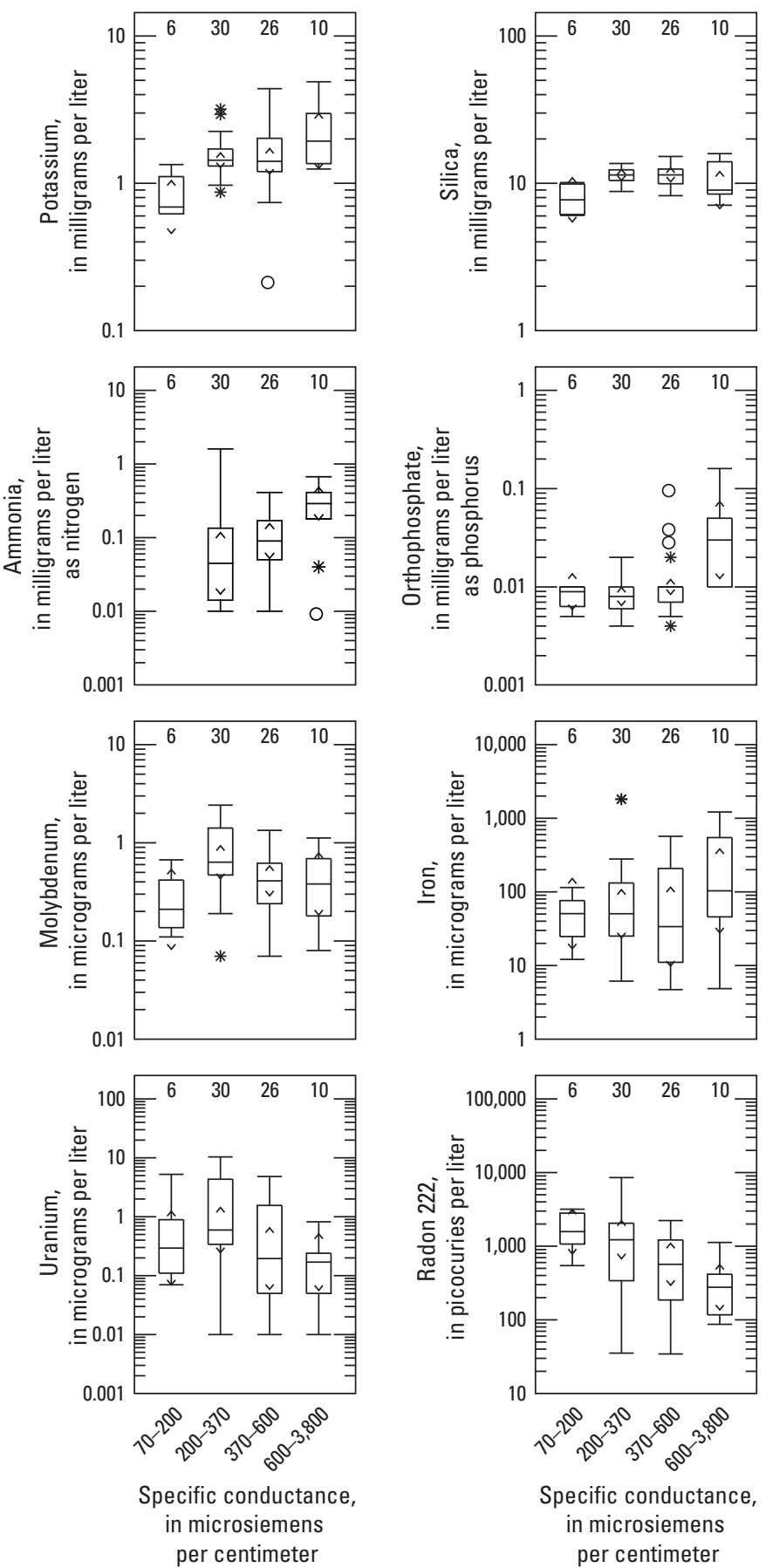

EXPLANATION

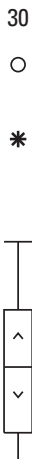

Number of samples

Outlier data value more than $\mathbf{3}$ times the interquartile range outside the quartile

* Outlier data value less than or equal to 3 and more than 1.5 times the interquartile range outside the quartile

\begin{tabular}{|l} 
Data value less than or equal to 1.5 times \\
the interquartile range outside the quartile \\
75th percentile \\
$\begin{array}{l}\text { Upper notch } \\
\text { Median } \\
\text { Lower notch } \\
\text { 25th percentile }\end{array}$
\end{tabular}



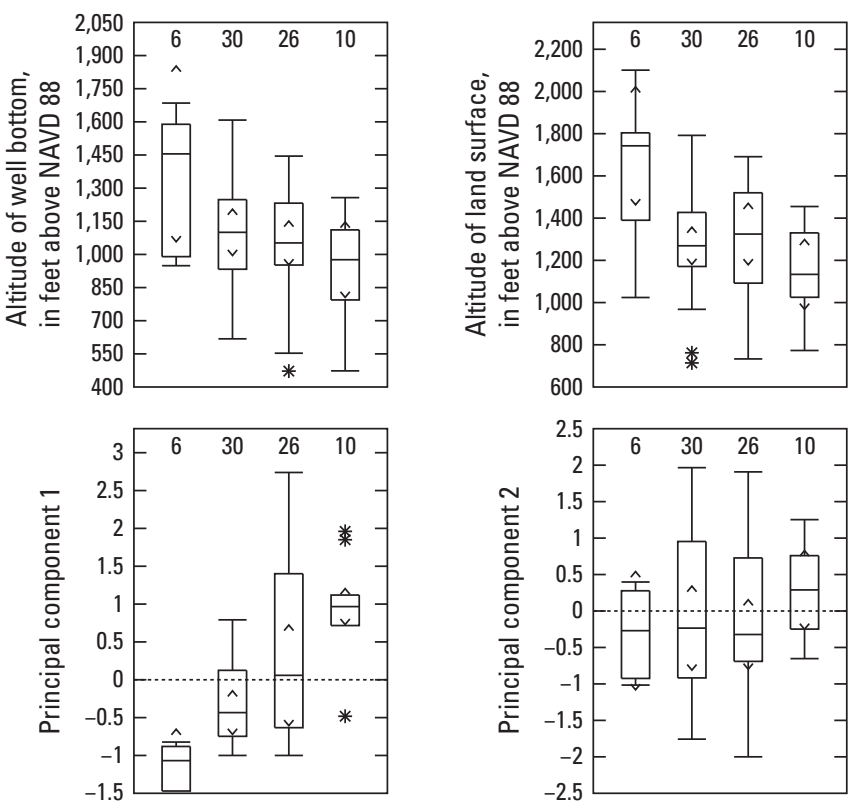

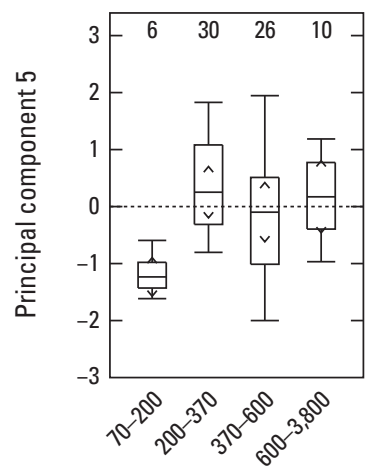

Specific conductance, in microsiemens per centimeter

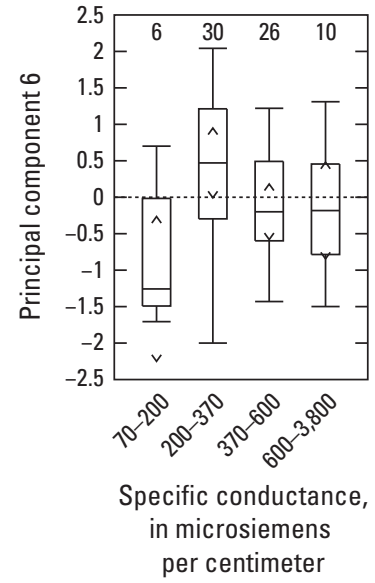

Figure 4.2. Composition of 72 groundwater samples from Bradford County, Pennsylvania, 2016, classified by specific conductance $(\mathrm{SC})$ class interval. The $\mathrm{SC}$ range is indicated on the $\mathrm{x}$-axis $(70<\mathrm{SC}<200 \mu \mathrm{S} / \mathrm{cm}, \mathrm{n}=6 ; 200<\mathrm{SC}<370 \mu \mathrm{S} / \mathrm{cm}, \mathrm{n}=30$; $370<\mathrm{SC}<600 \mu \mathrm{S} / \mathrm{cm}, \mathrm{n}=26 ; 600<\mathrm{SC}<3,800 \mu \mathrm{S} / \mathrm{cm}, \mathrm{n}=10$ ). $(<$, less than; $\mu \mathrm{S} / \mathrm{cm}$, microsiemens per centimeter; $\mathrm{n}$, number of samples; see table 3.1 for descriptions of principal components)Continued
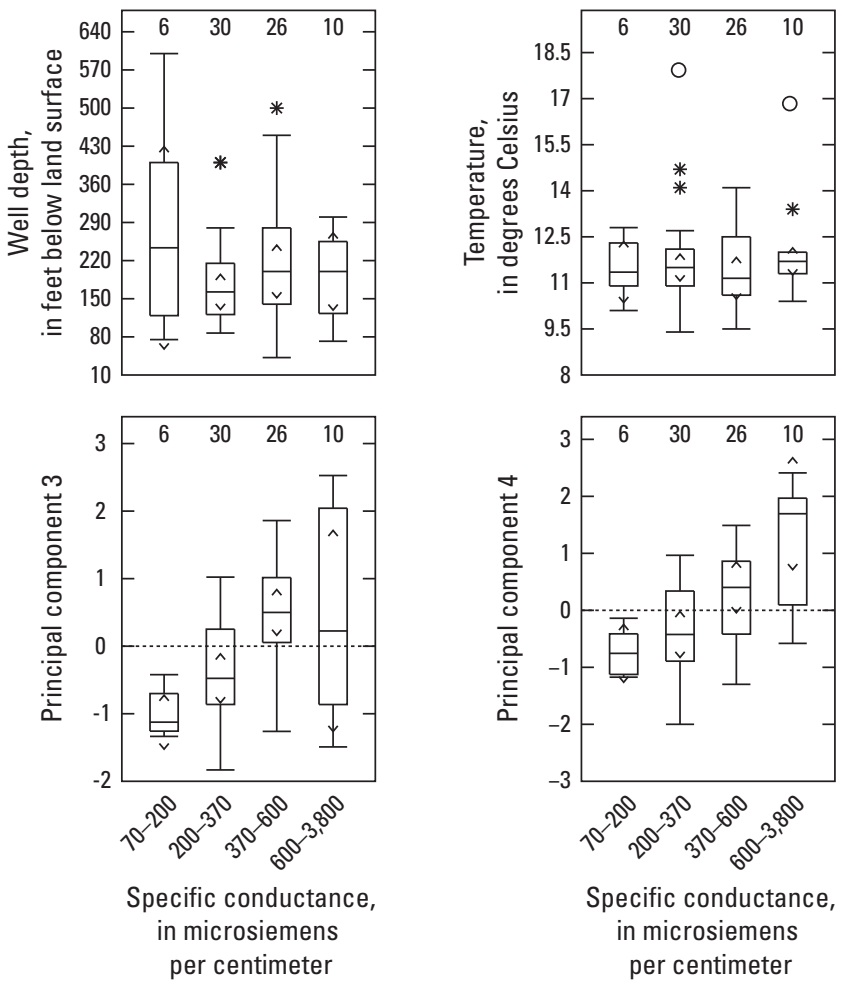

\section{EXPLANATION}

30 Number of samples

O Outlier data value more than 3 times the interquartile range outside the quartile

* Outlier data value less than or equal to 3 and more than 1.5 times the interquartile range outside the quartile

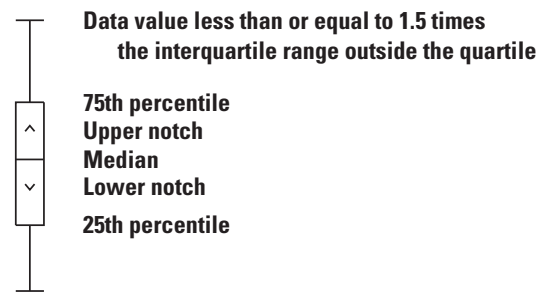



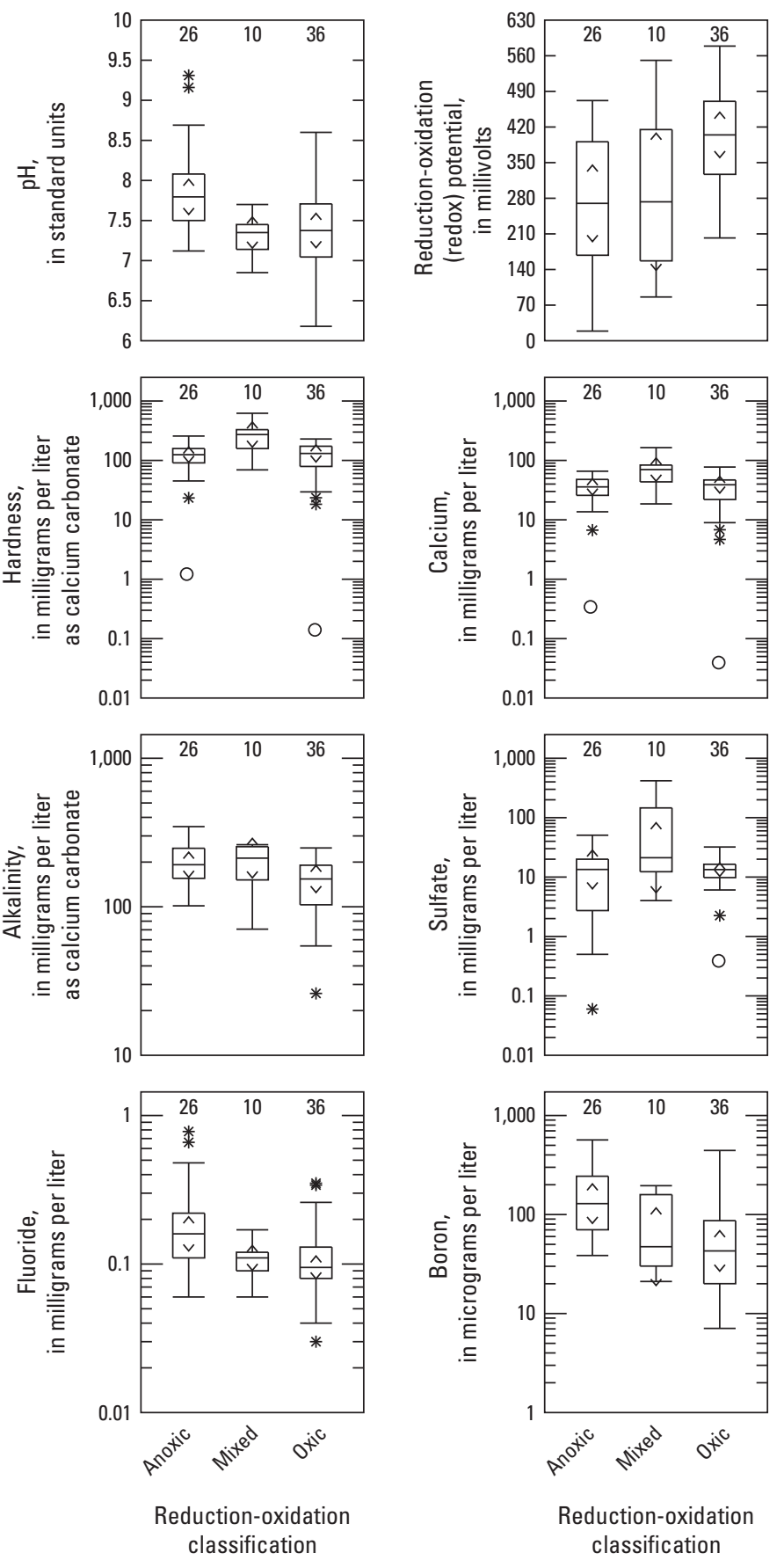

Reduction-oxidation classification
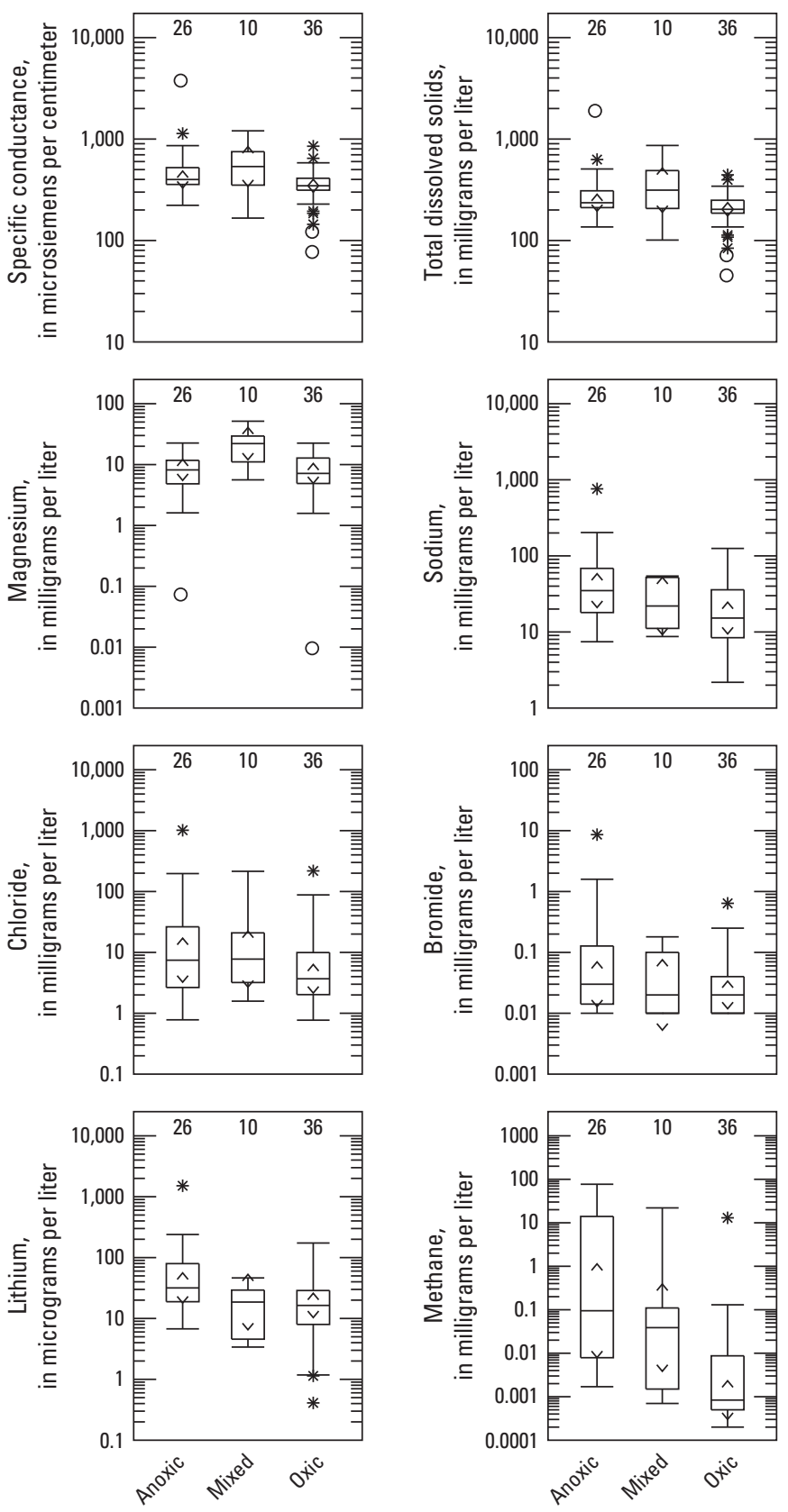

Reduction-oxidation classification

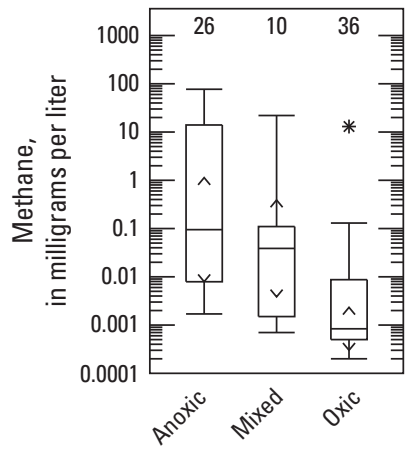

Reduction-oxidation classification
Figure 4.3. Composition of 72 groundwater samples from Bradford County, Pennsylvania, 2016, classified as anoxic $(n=26)$, mixed $(n=10)$, and oxic $(n=36)$ on the basis of dissolved oxygen concentration and other water-quality criteria of McMahon and Chapelle (2008). (n, number of samples; see table 3.1 for descriptions of principal components) 

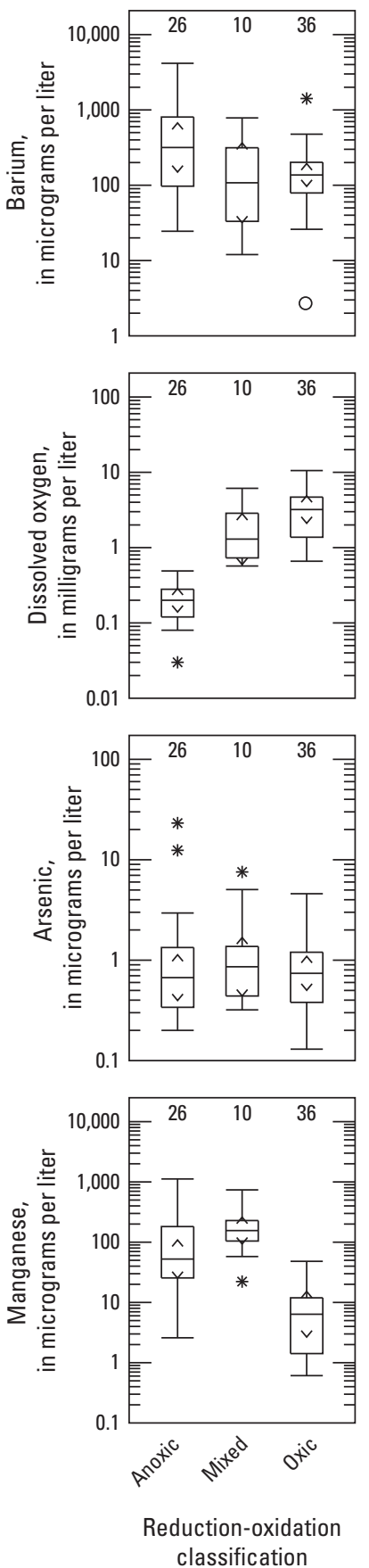
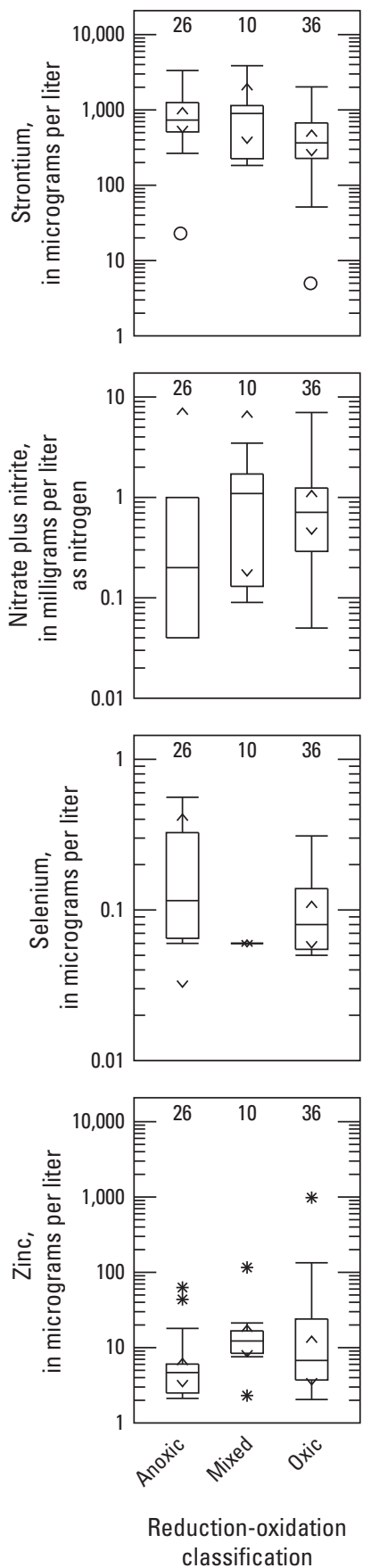

Figure 4.3. Composition of 72 groundwater samples from Bradford County, Pennsylvania, 2016, classified as anoxic $(n=26)$, mixed $(n=10)$, and oxic $(n=36)$ on the basis of dissolved oxygen concentration and other water-quality criteria of McMahon and Chapelle (2008). (n, number of samples; see table 3.1 for descriptions of principal components)-Continued
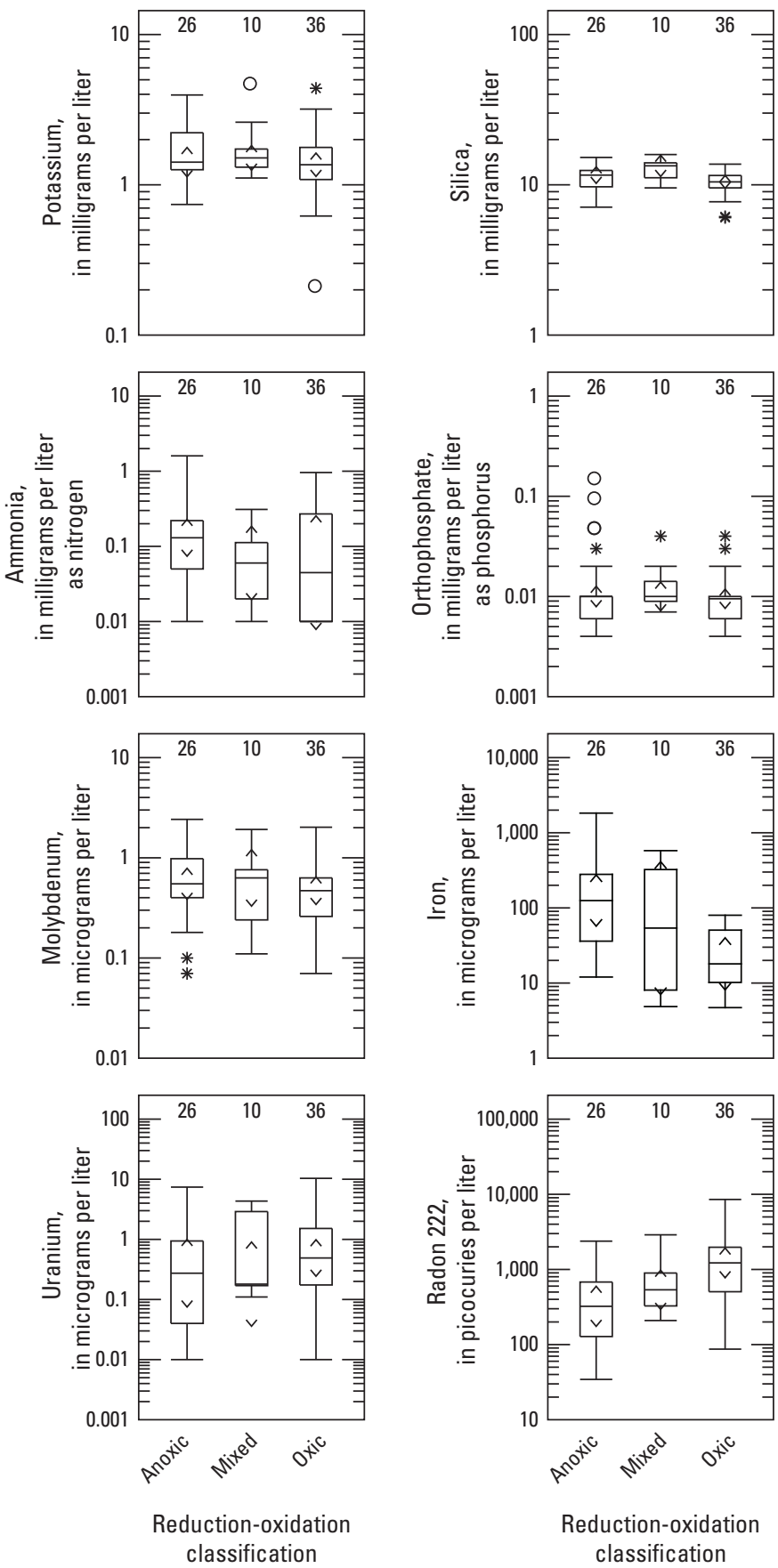

Reduction-oxidation classification

EXPLANATION

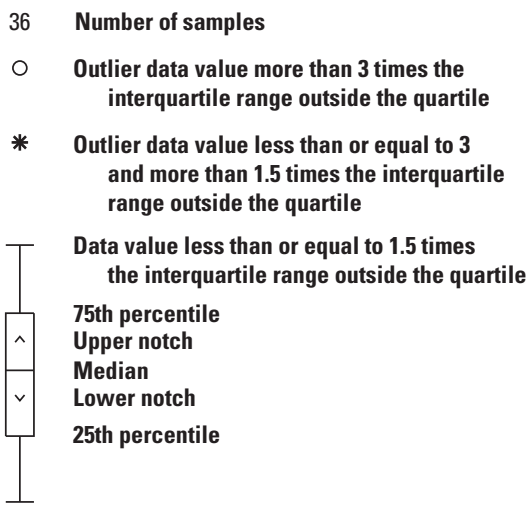



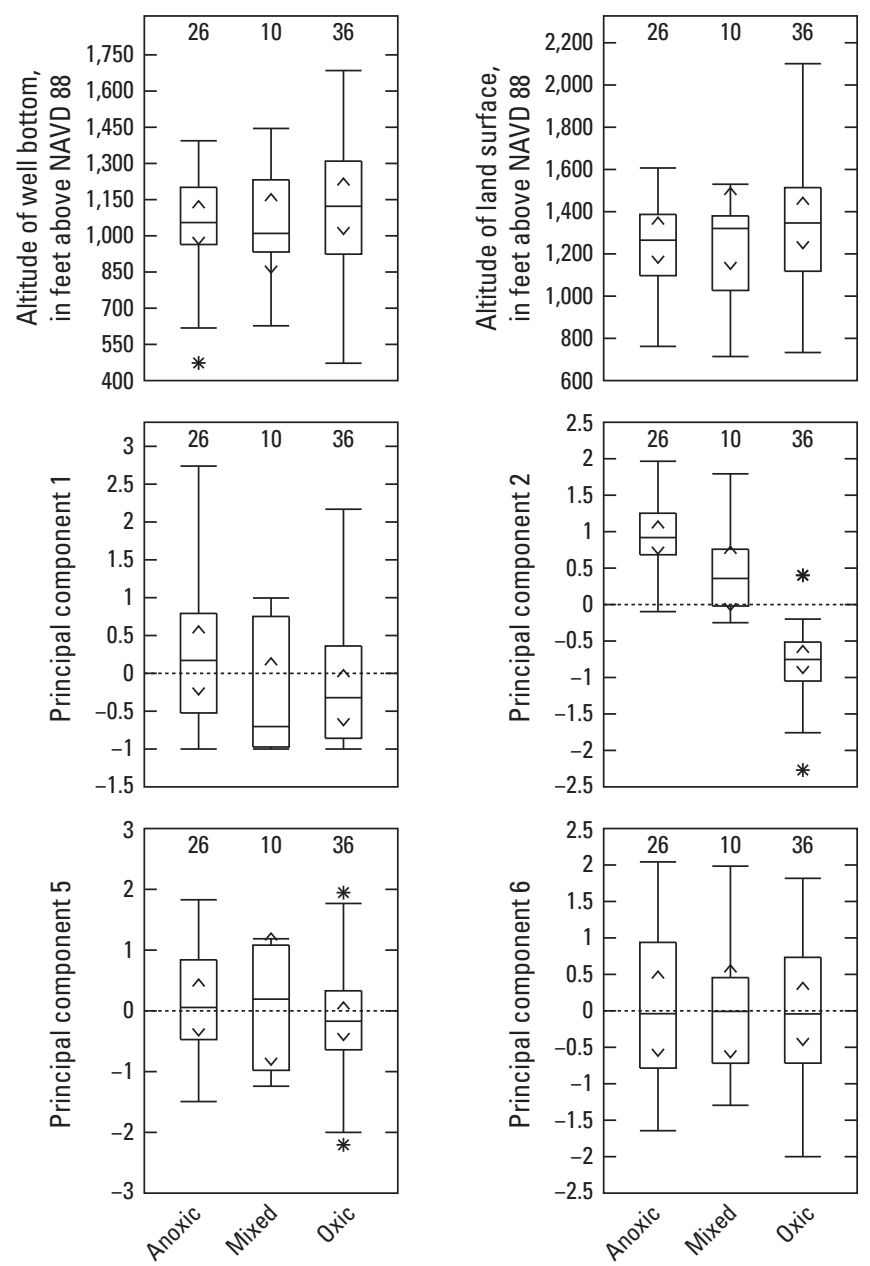

Reduction-oxidation classification
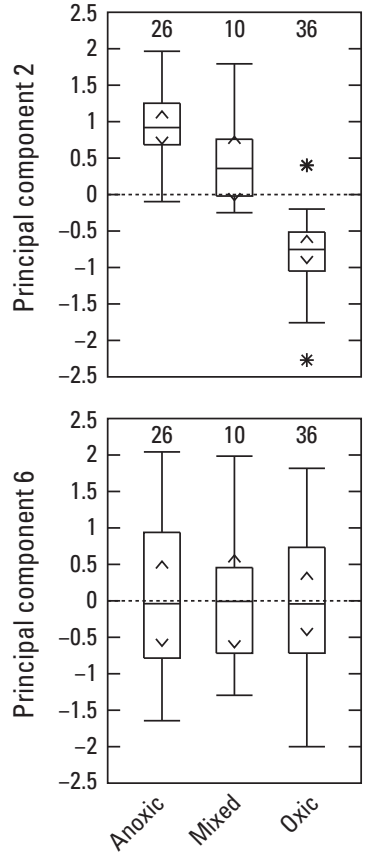

Reduction-oxidation classification

Figure 4.3. Composition of 72 groundwater samples from Bradford County, Pennsylvania, 2016, classified as anoxic $(n=26)$, mixed $(n=10)$, and oxic $(n=36)$ on the basis of dissolved oxygen concentration and other water-quality criteria of McMahon and Chapelle (2008). (n, number of samples; see table 3.1 for descriptions of principal components)-Continued
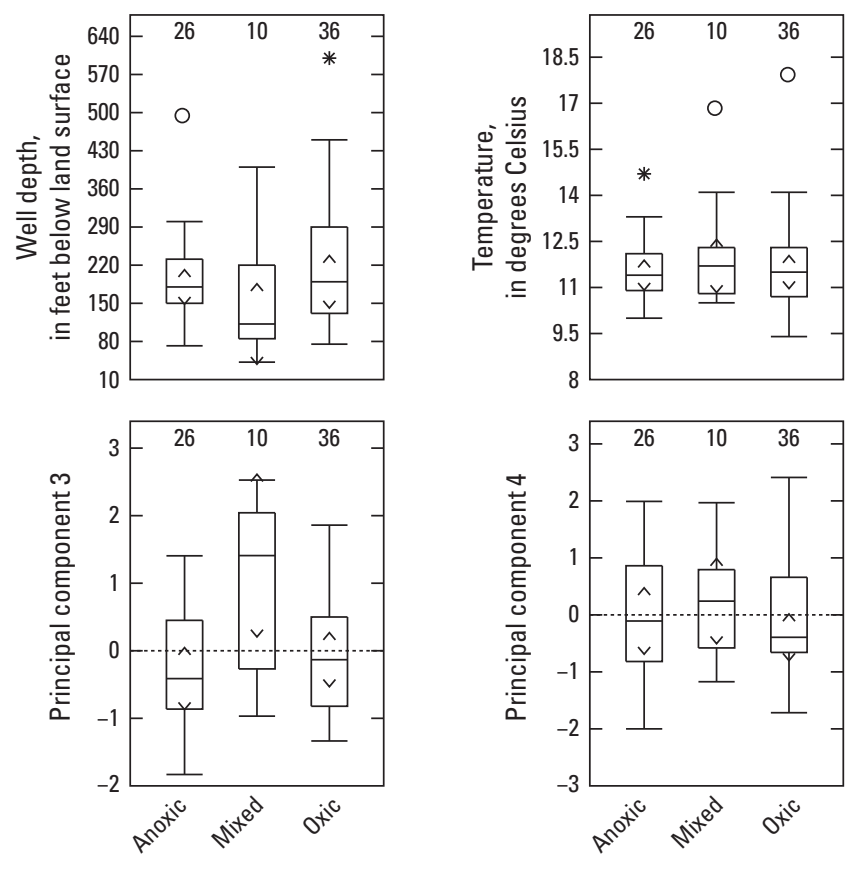

Reduction-oxidation classification
Reduction-oxidation classification

\section{EXPLANATION}

$36 \quad$ Number of samples

O Outlier data value more than 3 times the interquartile range outside the quartile

* Outlier data value less than or equal to 3 and more than 1.5 times the interquartile range outside the quartile

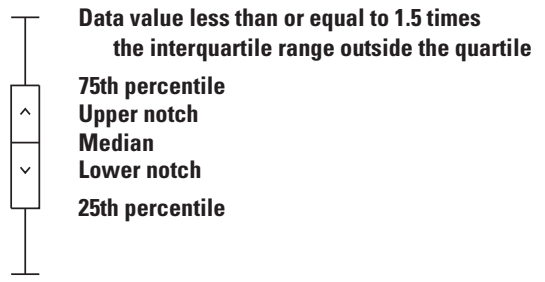


Appendix 4.
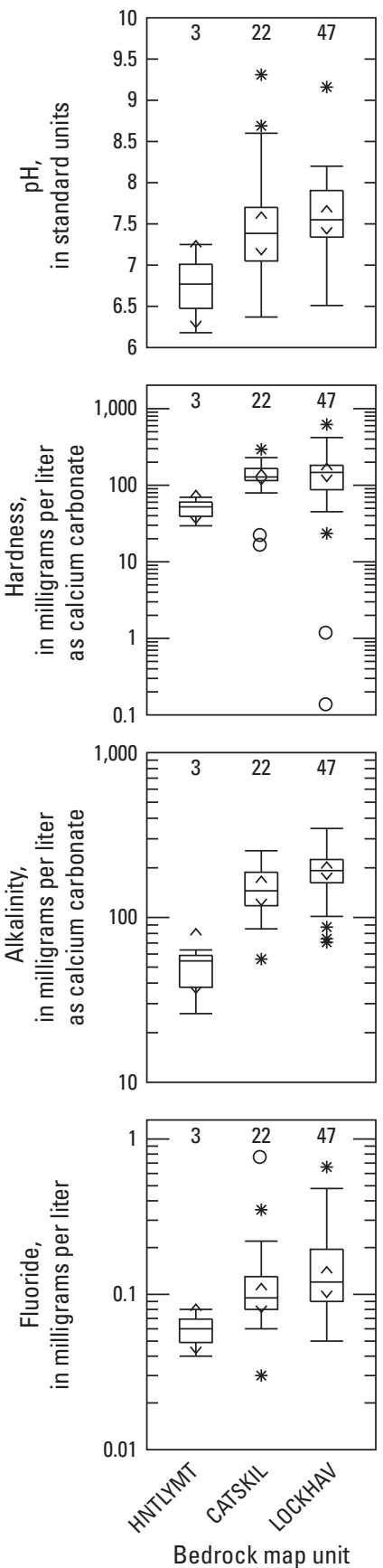
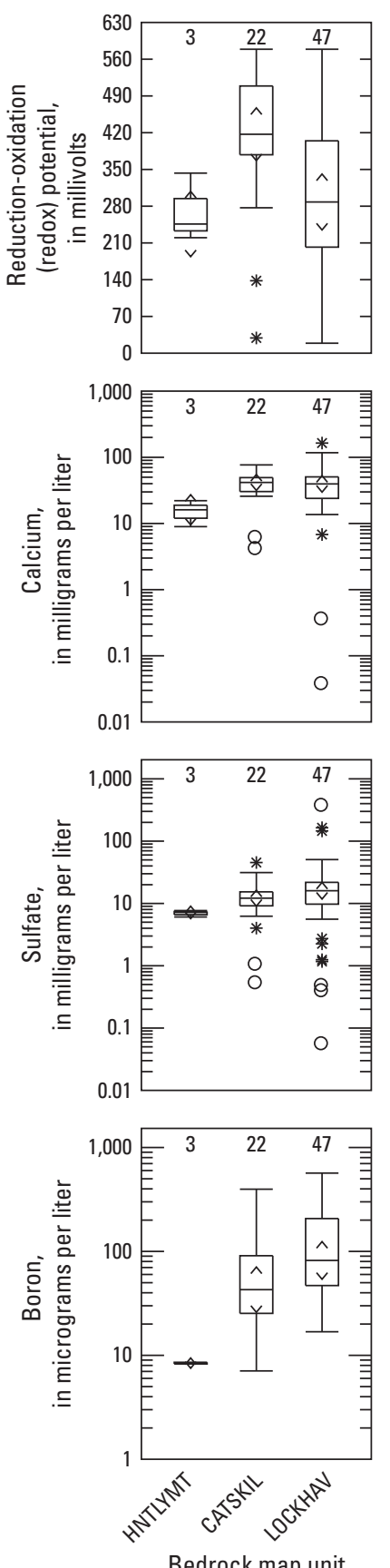

Bedrock map unit
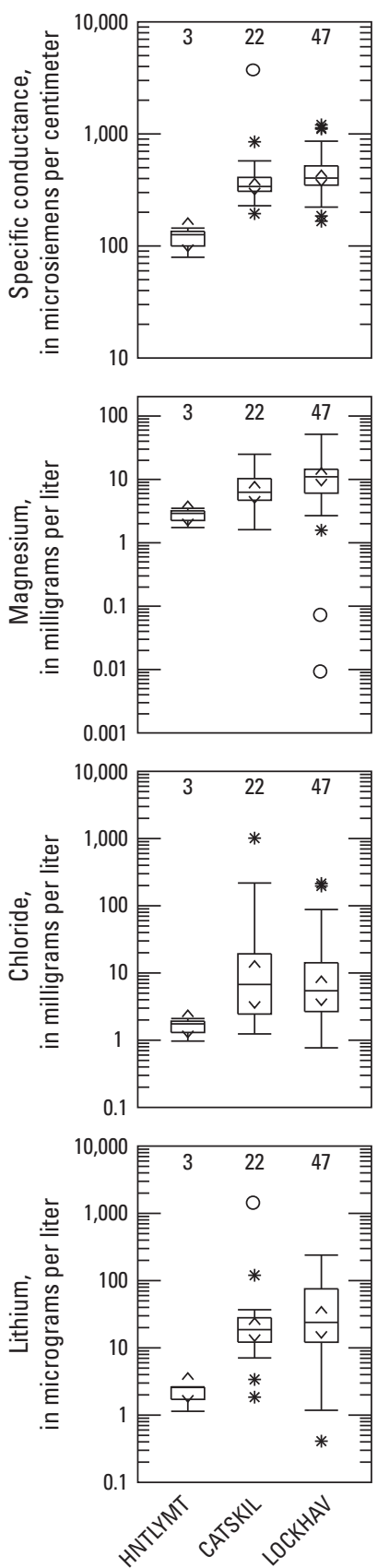

Bedrock map unit
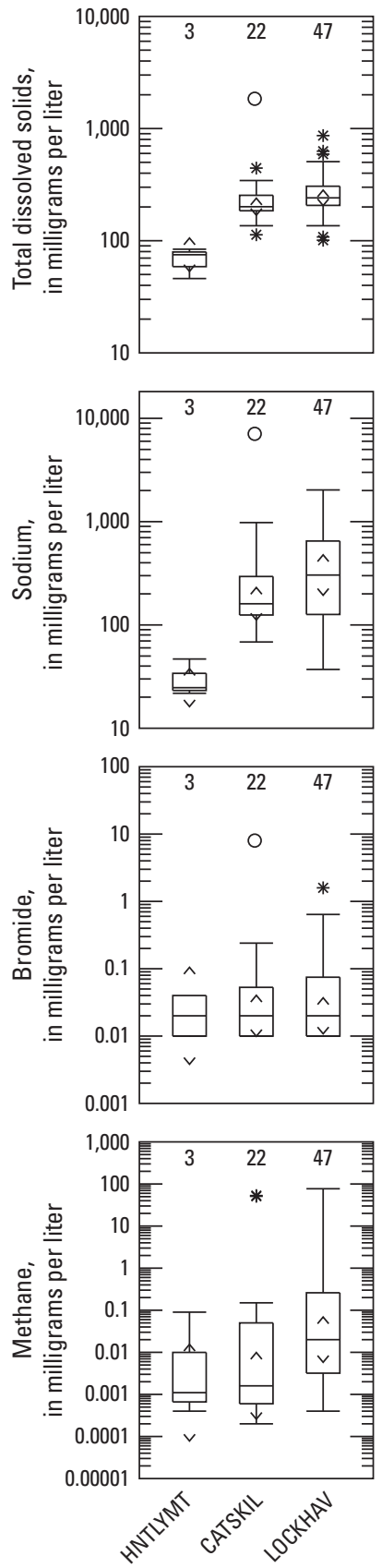

Bedrock map unit

EXPLANATION

Figure 4.4. Composition of 72 groundwater samples from Bradford County, Pennsylvania, 2016, classified on the basis of bedrock map unit identified by Pennsylvania Bureau of Topographic and Geologic Survey (2001) as Huntley Mountain Formation (HNTLYMT; $n=3$ ), Catskill Formation (CATSKIL; $n=22$ ), and Lock Haven Formation (LOCKHAV; $n=47)$. ( $n$, number of samples; see table 3.1 for descriptions of principal components)

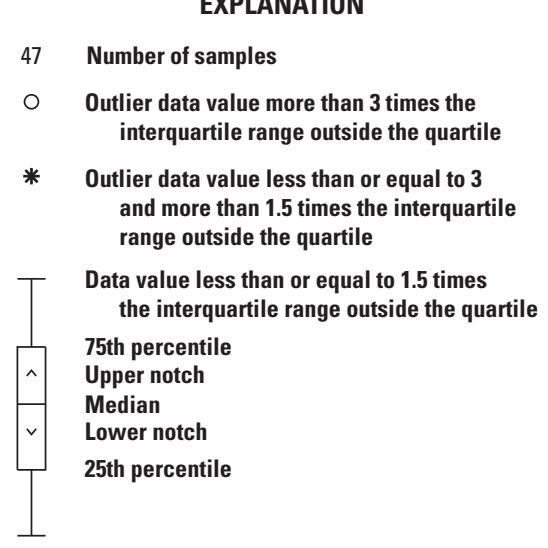



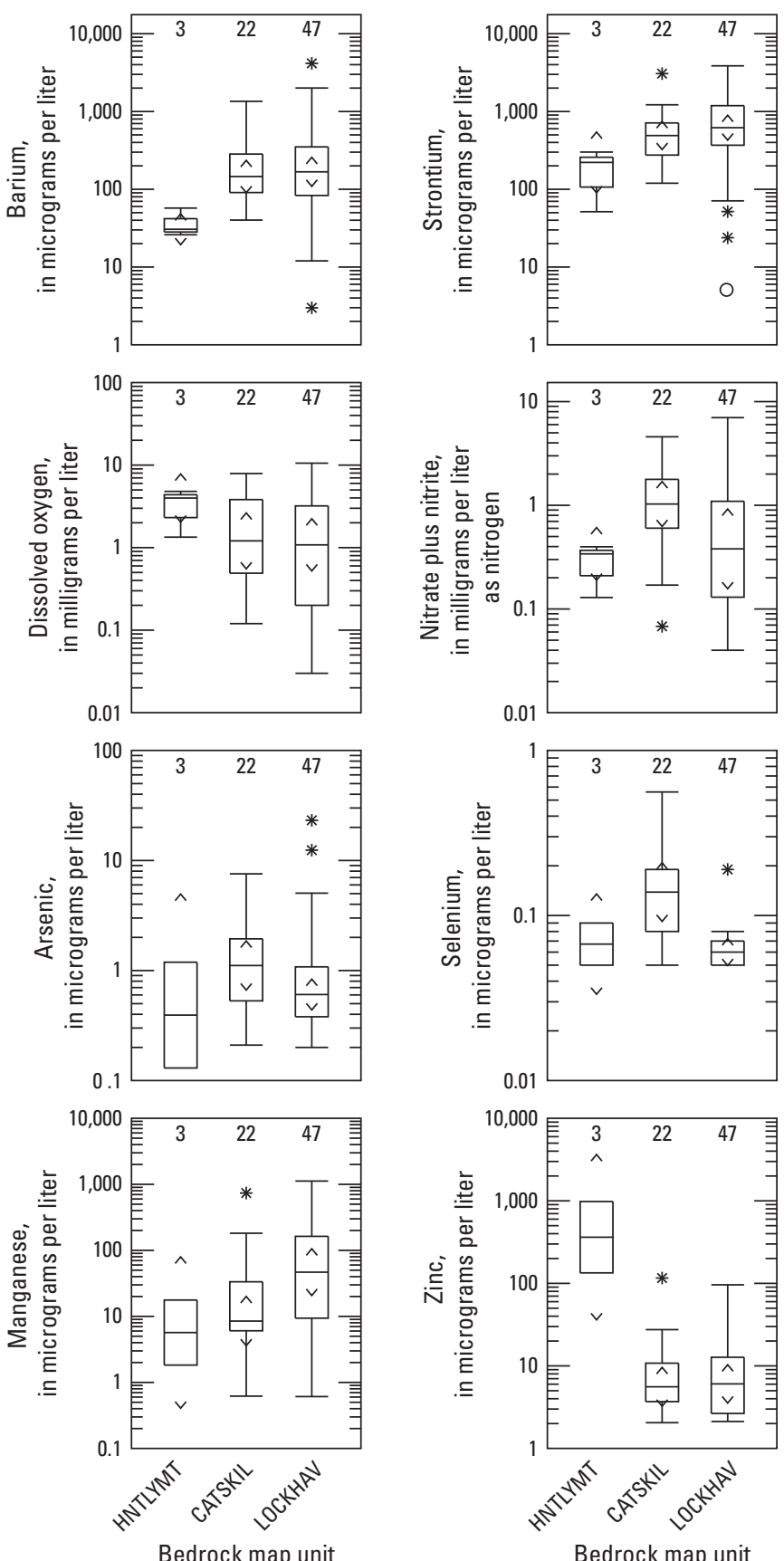

Figure 4.4. Composition of 72 groundwater samples from Bradford County, Pennsylvania, 2016, classified on the basis of bedrock map unit identified by Pennsylvania Bureau of Topographic and Geologic Survey (2001) as Huntley Mountain Formation (HNTLYMT; $n=3$ ), Catskill Formation (CATSKIL; $n=22$ ), and Lock Haven Formation (LOCKHAV; $n=47$ ). (n, number of samples; see table 3.1 for descriptions of principal components)Continued

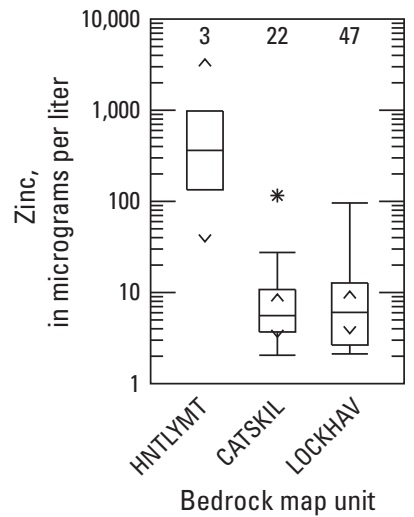

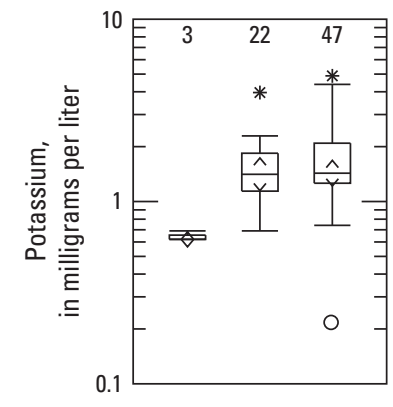
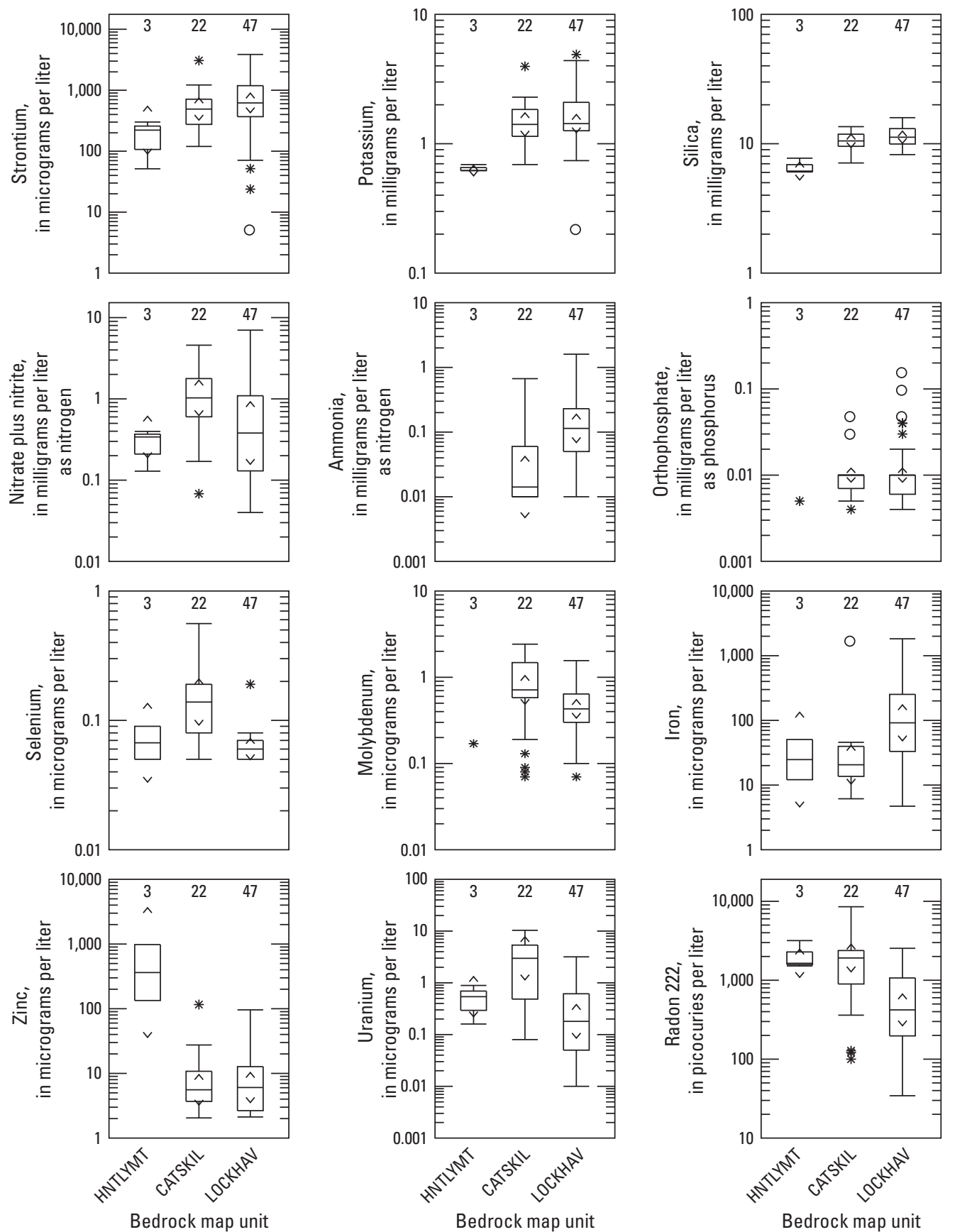

Bedrock map unit

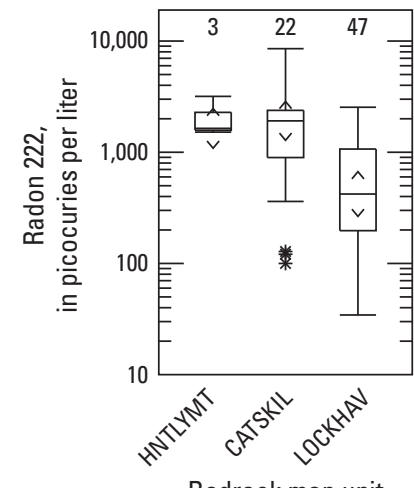

EXPLANATION

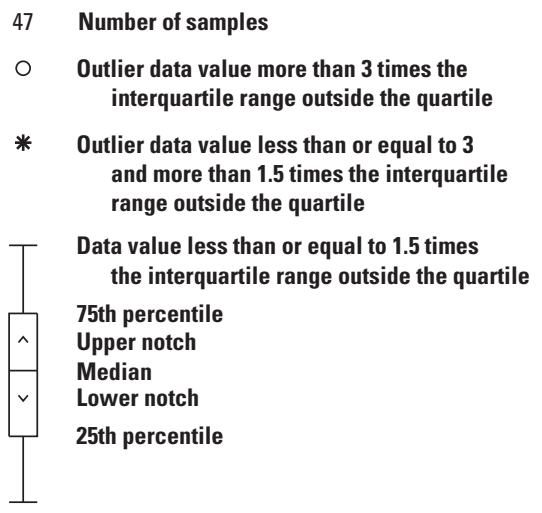



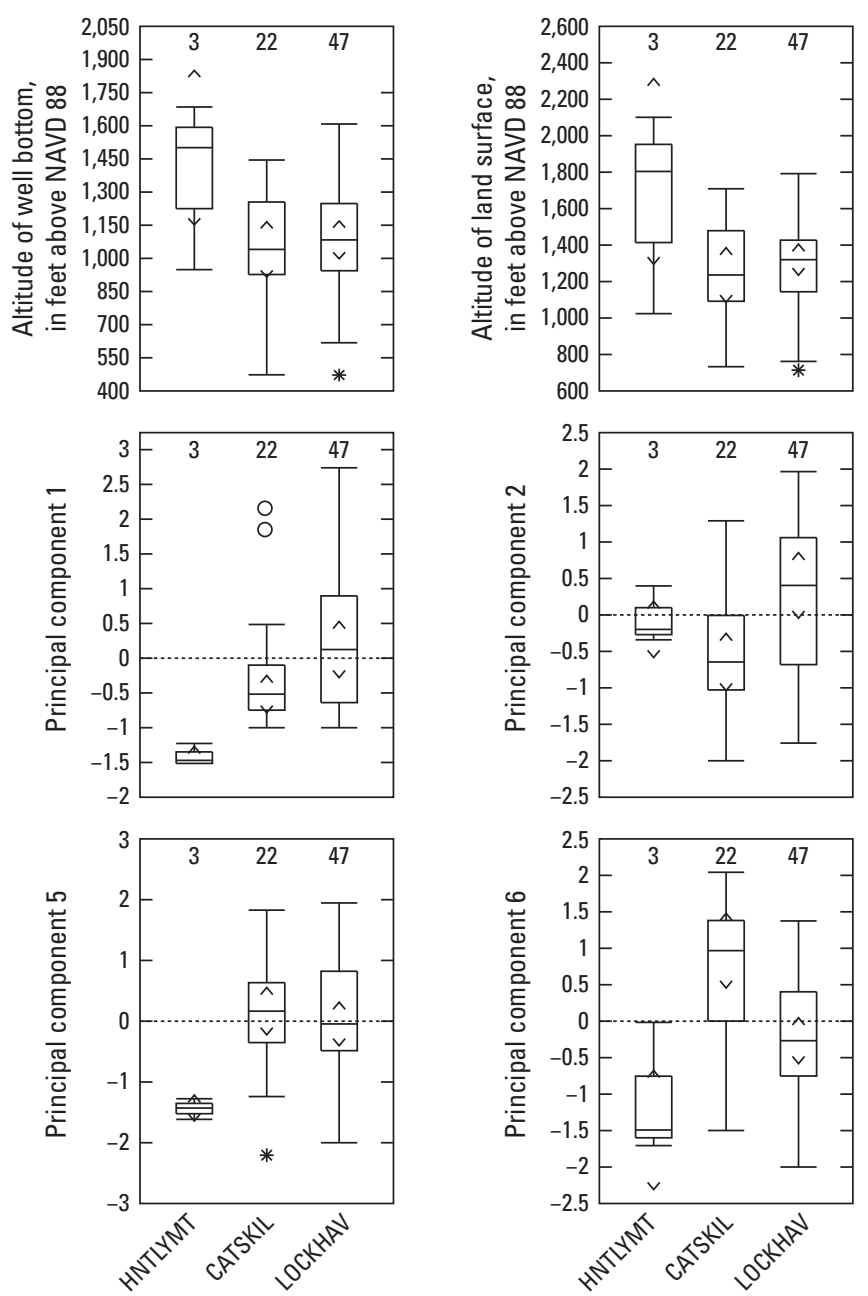

Bedrock map unit

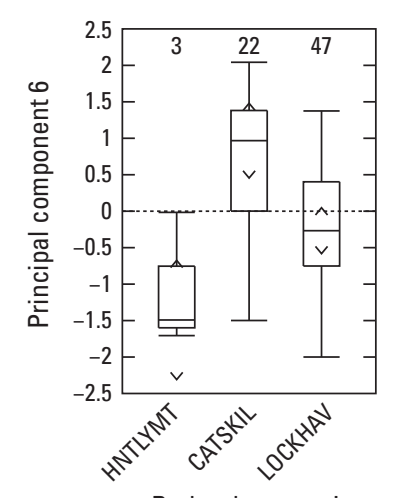

Bedrock map unit

Figure 4.4. Composition of 72 groundwater samples from Bradford County, Pennsylvania, 2016, classified on the basis of bedrock map unit identified by Pennsylvania Bureau of Topographic and Geologic Survey (2001) as Huntley Mountain Formation (HNTLYMT; $n=3$ ), Catskill Formation (CATSKIL; $n=22$ ), and Lock Haven Formation (LOCKHAV; $n=47$ ). (n, number of samples; see table 3.1 for descriptions of principal components)Continued
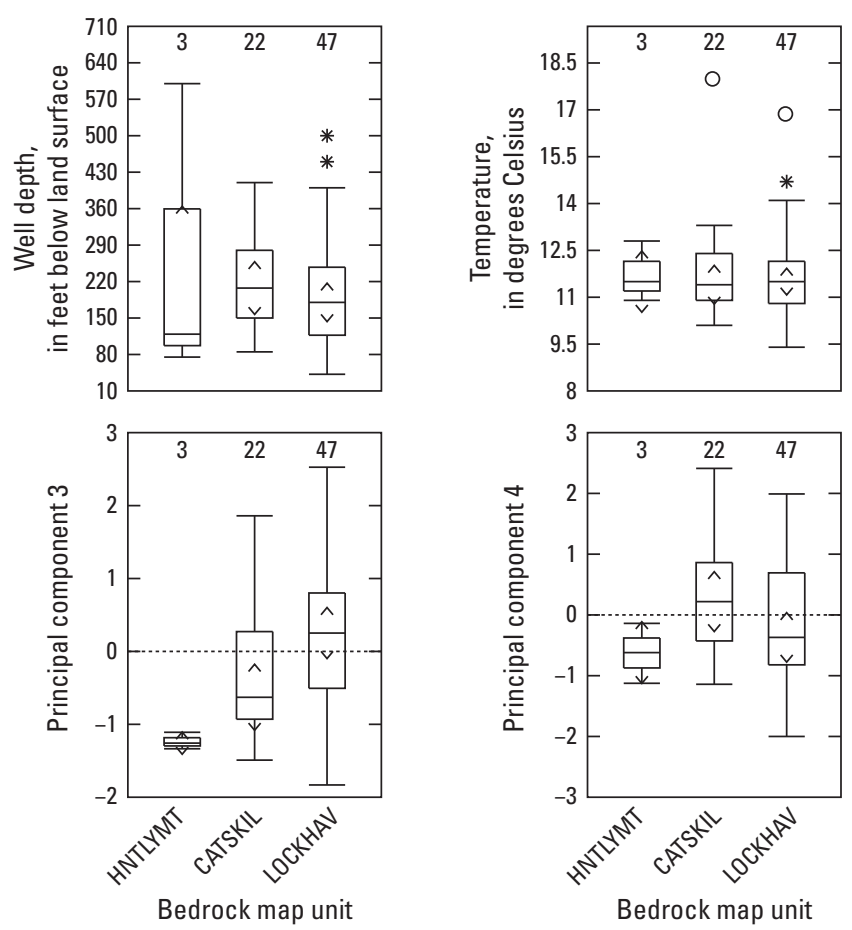

EXPLANATION

$47 \quad$ Number of samples

O Outlier data value more than 3 times the interquartile range outside the quartile

* Outlier data value less than or equal to 3 and more than 1.5 times the interquartile range outside the quartile

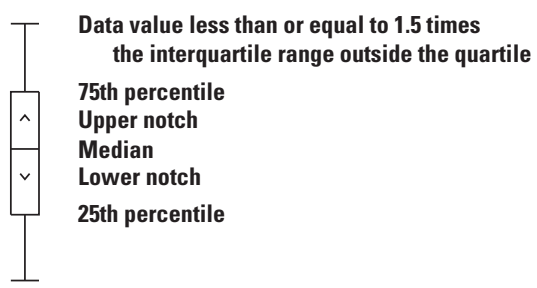



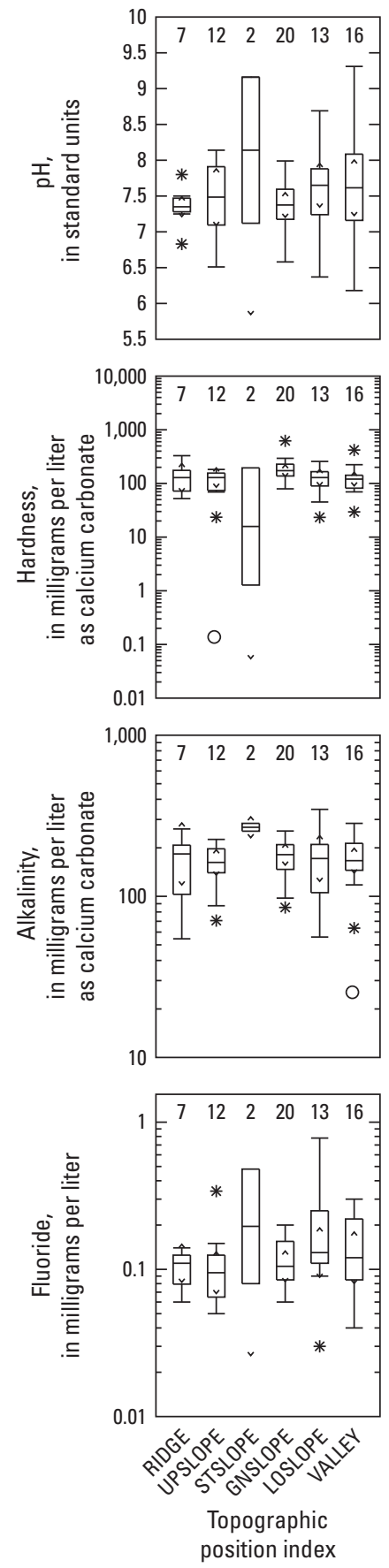
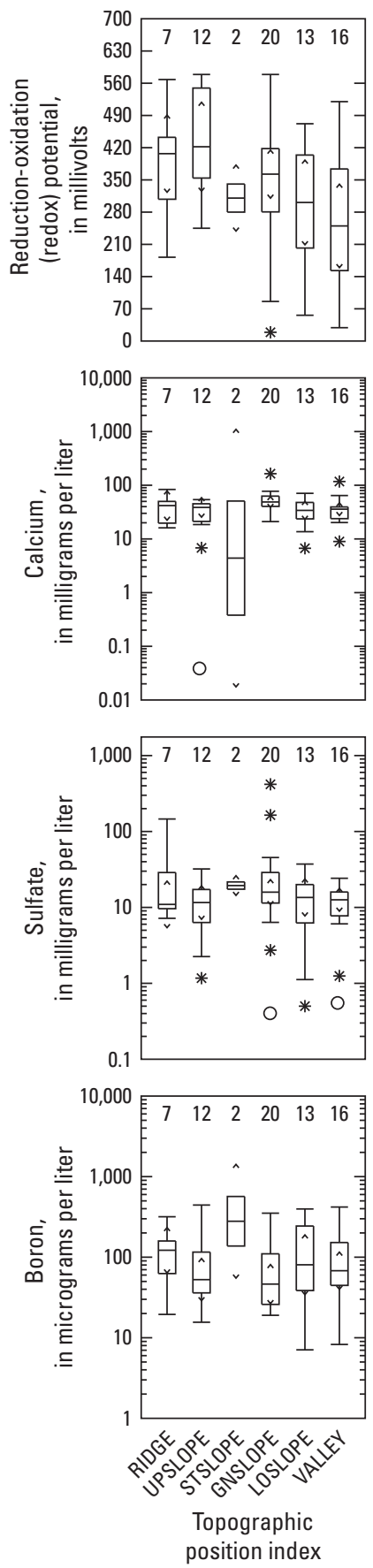

Figure 4.5. Composition of 72 groundwater samples from Bradford County, Pennsylvania, 2016, classified on the basis of topographic position index (TPI). The TPI classes are ridge (RIDGE; $n=7$ ), upper slope (UPSLOPE; $n=12$ ), steep slope (STSLOPE; $n=2$ ), gentle slope (GNSLOPE; $n=20$ ), lower slope (LOSLOPE; $n=13$ ), and valley (VALLEY; $n=16$ ). ( $n$, number of samples; see table 3.1 for descriptions of principal components)
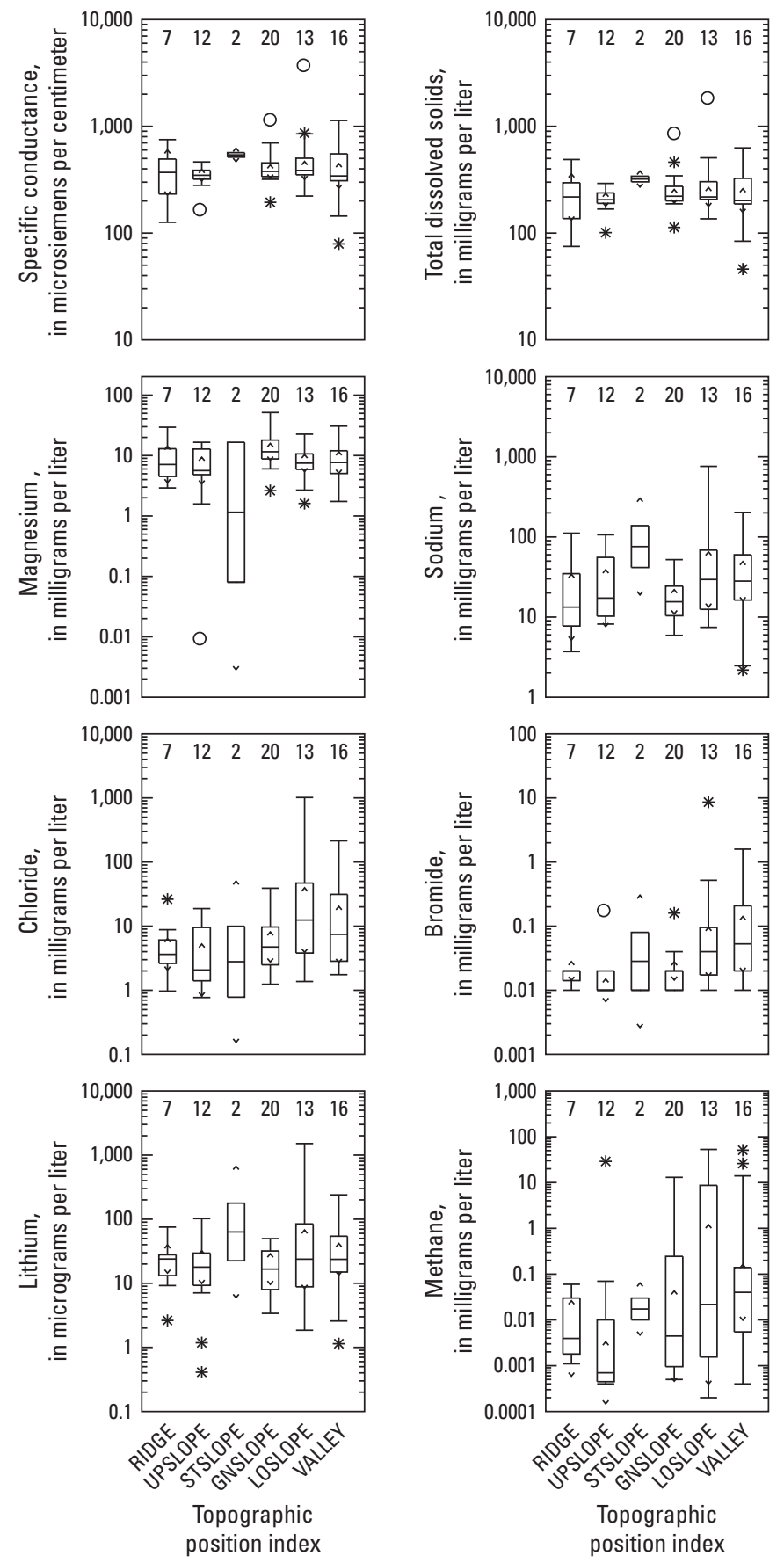

EXPLANATION

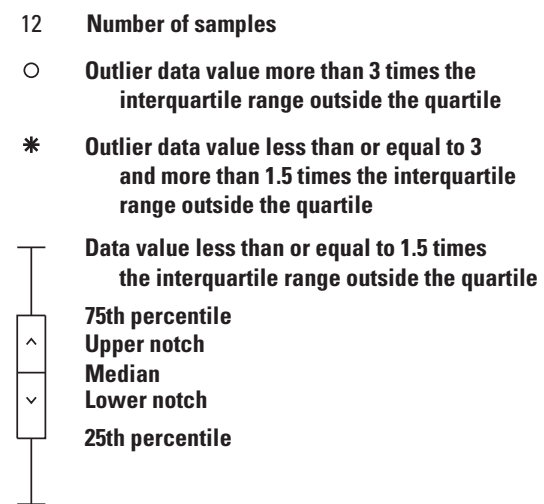



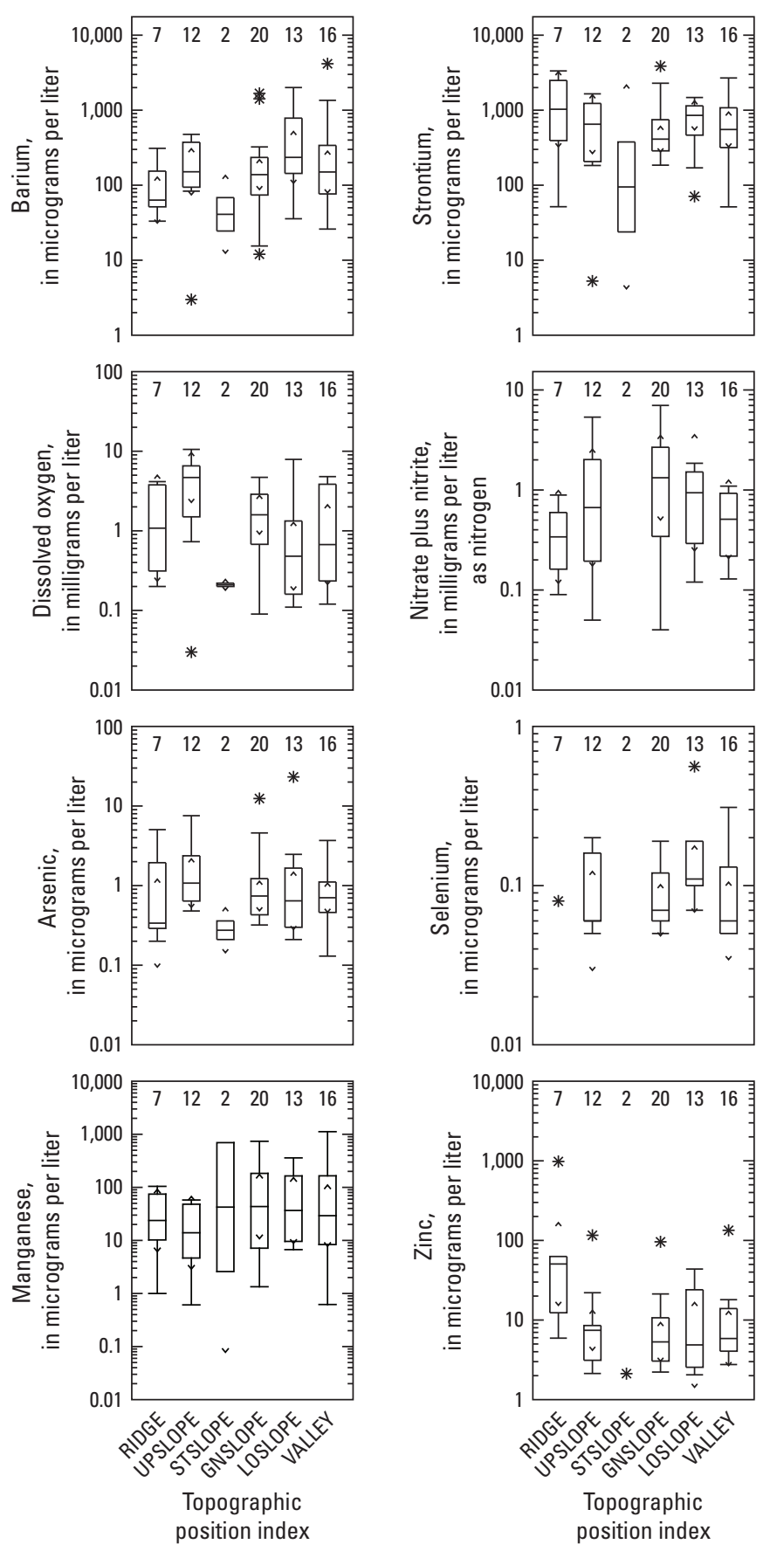

Figure 4.5. Composition of 72 groundwater samples from Bradford County, Pennsylvania, 2016, classified on the basis of topographic position index (TPI). The TPI classes are ridge (RIDGE; $n=7$ ), upper slope (UPSLOPE; $n=12$ ), steep slope (STSLOPE; $n=2$ ), gentle slope (GNSLOPE; $n=20$ ), lower slope (LOSLOPE; $n=13$ ), and valley (VALLEY; $n=16$ ). ( $n$, number of samples; see table 3.1 for descriptions of principal components)Continued
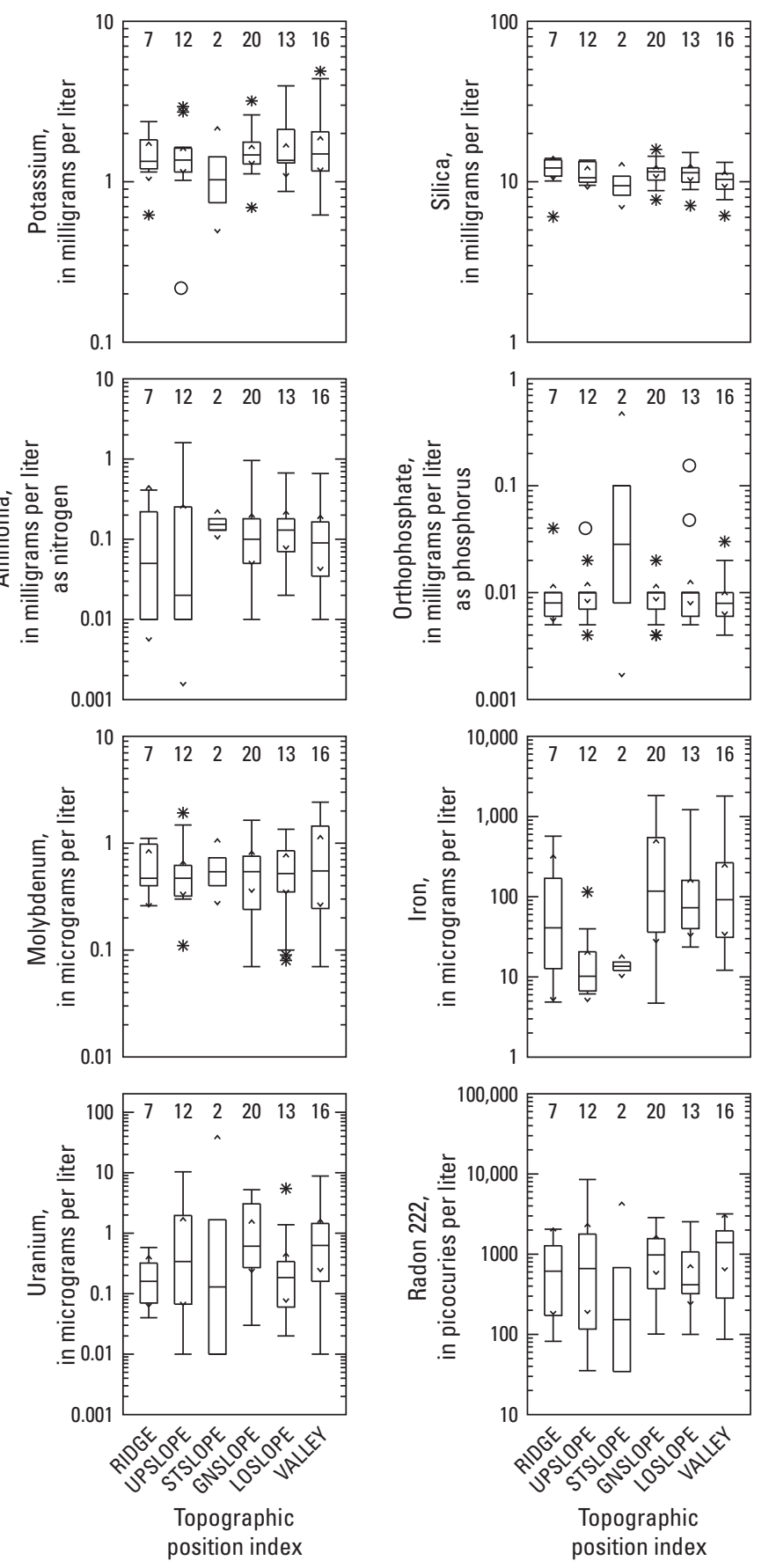

EXPLANATION

12 Number of samples

O Outlier data value more than 3 times the interquartile range outside the quartile

* Outlier data value less than or equal to 3 and more than 1.5 times the interquartile range outside the quartile

Data value less than or equal to 1.5 times the interquartile range outside the quartile 75th percentile

Upper notch

Median

Lower notch

25th percentile 

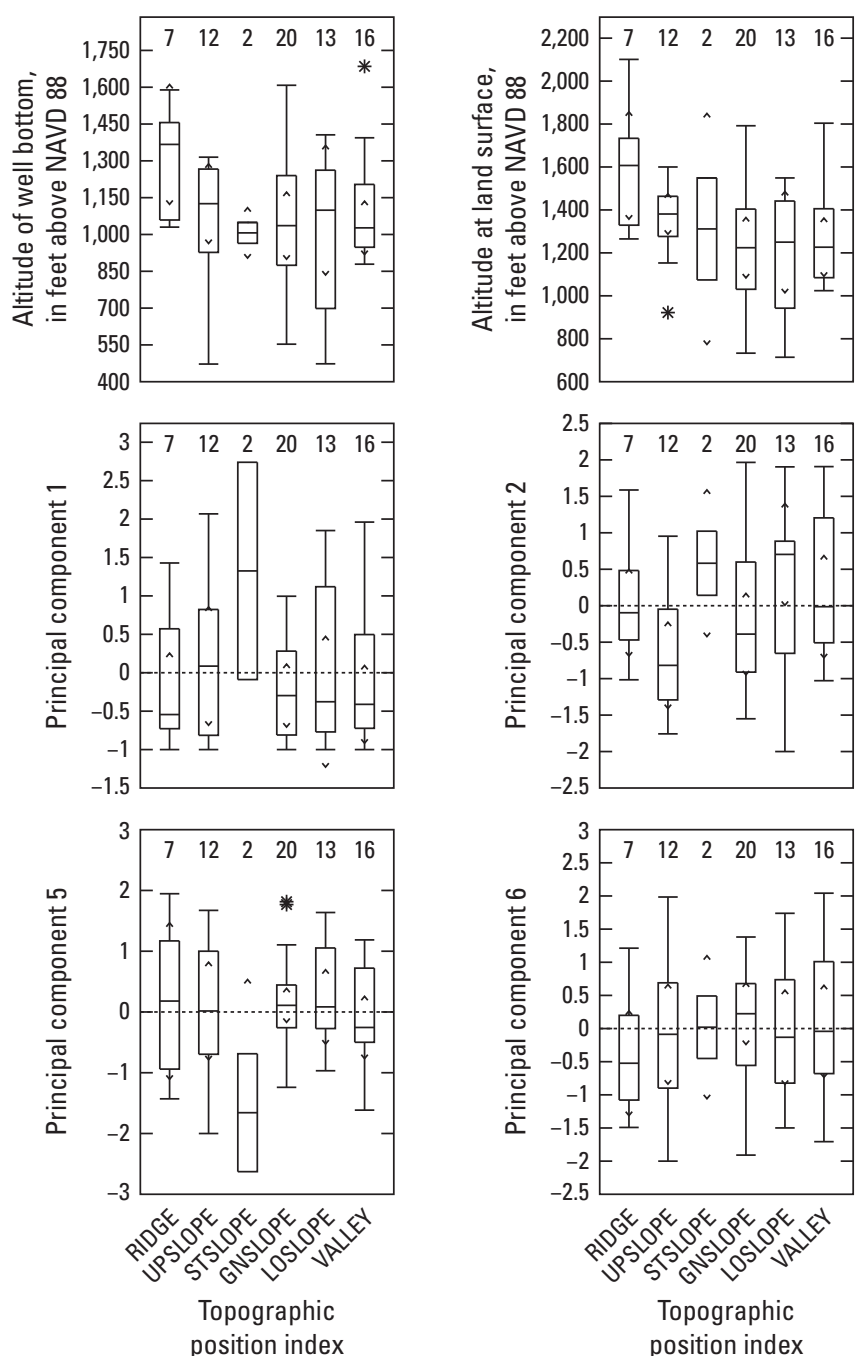

Figure 4.5. Composition of 72 groundwater samples from Bradford County, Pennsylvania, 2016, classified on the basis of topographic position index (TPI). The TPI classes are ridge (RIDGE; $n=7$ ), upper slope (UPSLOPE; $n=12$ ), steep slope (STSLOPE; $n=2$ ), gentle slope (GNSLOPE; $n=20$ ), lower slope (LOSLOPE; $n=13$ ), and valley (VALLEY; $n=16$ ). ( $n$, number of samples; see table 3.1 for descriptions of principal components) Continued
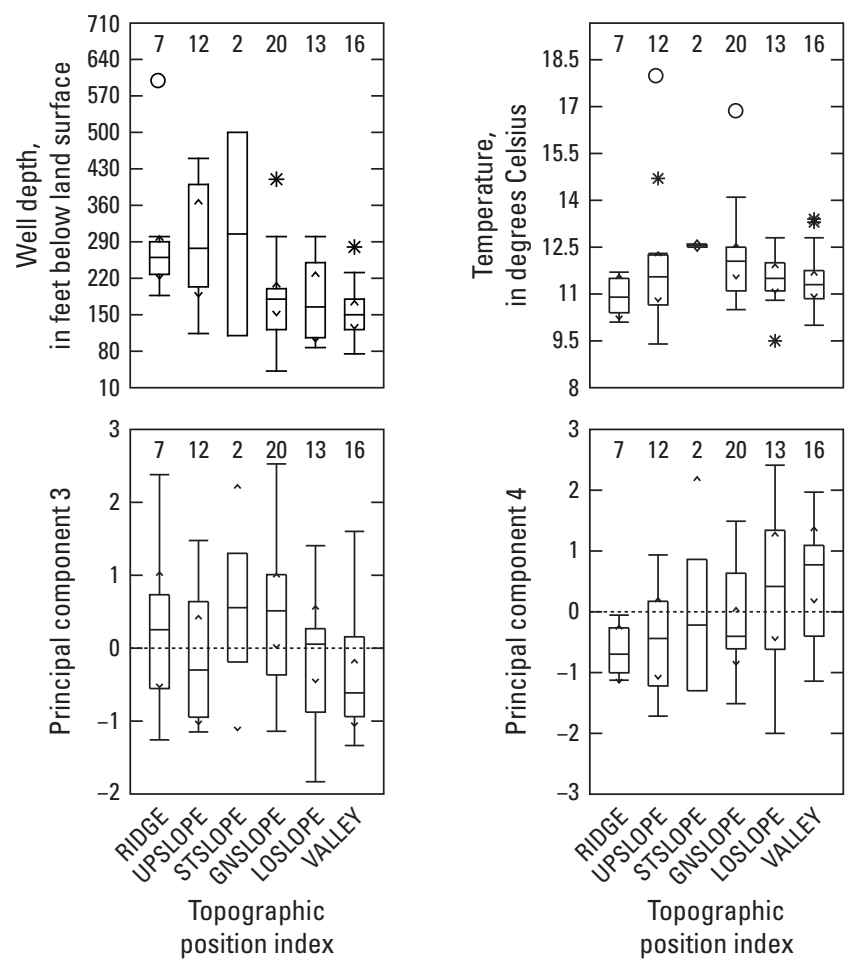

EXPLANATION

2 Number of samples

O Outlier data value more than 3 times the interquartile range outside the quartile

* Outlier data value less than or equal to 3 and more than 1.5 times the interquartile range outside the quartile

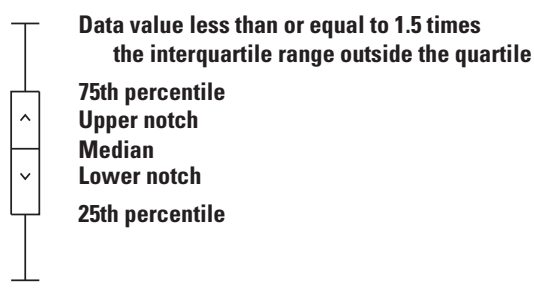


For additional information, contact:

Director, Pennsylvania Water Science Center

U.S. Geological Survey

215 Limekiln Road

New Cumberland, PA 17070

or visit our website at:

https://pa.water.usgs.gov/

Publishing support provided by the West Trenton Publishing Service Center 
